University of Tennessee Health Science Center

UTHSC Digital Commons

$12-2013$

\title{
Toll-like Receptors 2 and 9 Are Important for Innate Immune Cell Activation and Recruitment in Hypersensitivity Pneumonitis
}

Kelly J. Andrews

University of Tennessee Health Science Center

Follow this and additional works at: https://dc.uthsc.edu/dissertations

Part of the Bacterial Infections and Mycoses Commons, Biological Phenomena, Cell Phenomena, and Immunity Commons, Medical Immunology Commons, Medical Microbiology Commons, and the Respiratory Tract Diseases Commons

\section{Recommended Citation}

Andrews, Kelly J. , "Toll-like Receptors 2 and 9 Are Important for Innate Immune Cell Activation and Recruitment in Hypersensitivity Pneumonitis" (2013). Theses and Dissertations (ETD). Paper 14. http://dx.doi.org/10.21007/etd.cghs.2013.0015.

This Thesis is brought to you for free and open access by the College of Graduate Health Sciences at UTHSC Digital Commons. It has been accepted for inclusion in Theses and Dissertations (ETD) by an authorized administrator of UTHSC Digital Commons. For more information, please contact jwelch30@uthsc.edu. 


\title{
Toll-like Receptors 2 and 9 Are Important for Innate Immune Cell Activation and Recruitment in Hypersensitivity Pneumonitis
}

\begin{abstract}
Hypersensitivity pneumonitis (HP) is an interstitial lung disease caused by repeated inhalation of environmental antigens. The disease is characterized by alveolitis and granuloma formation; however, some patients develop chronic HP (CHP), a restrictive lung disease characterized by fibrosis. Previous studies revealed that neutrophils are recruited into the lung via a MyD88- dependent pathway and regulate disease severity through cytokine production. Toll-like receptors (TLRs) 2 and 9 recognize conserved molecular patterns present in bacteria, and signal through the adaptor molecule MyD88. The goal of my project is to investigate the role of TLRs 2 and 9 in the pathogenesis of HP during the acute phase, granulomatous phase, and chronic phase of the disease. Using the S. rectivirgula (SR) animal model of $\mathrm{HP}$, our studies indicated that individually, TLRs 2 and 9 contributed to neutrophil recruitment. We generated TLR2/9 double knockout (TLR2/9-/- ) mice to determine the extent to which TLRs 2 and 9 cooperate in neutrophil recruitment during HP. During the acute response, TLR2/9-/- mice exposed to SR demonstrated a significant reduction in neutrophil recruitment after a single exposure to SR compared to C57BL/6 mice (WT). The reduction in neutrophils was associated with a reduction in the expression of the neutrophil chemokine CXCL2 and inflammatory cytokines TNF $\alpha$ and IL-6 in the BALF. Disease severity in $\mathrm{HP}$ is associated with granuloma formation and a Th17 response. After 3 weeks exposure to SR, our results demonstrate a decrease in IL-17 and IL-22 mRNA expression and a corresponding decrease in the percentage of Th17 cells in TLR2/9-/- mice compared to WT and single knockout (SKO) mice. The decrease in Th17 cells in TLR2/9-/- mice was not associated with a significant increase in T regulatory (Treg) cells or a switch to a Th1 response. In addition, TLR2-/- and TLR2/9-/- mice have significantly fewer activated CD4+ Th cells. Interstitial macrophages from TLR2/9-/- mice have decreased costimulatory molecule and $\mathrm{MHCll}$ expression suggesting they are deficient in T cell activation. However, TLR2-/- , TLR9-/- , and TLR2/9-/- still form granulomas in the lung. To determine the extent to which TLRs 2 and 9 contributed to the development of CHP, WT and TLR2/9-/- mice were intranasally exposed to SR three times/week for 15 weeks. WT and TLR2/9-/- mice developed a neutrophilic and lymphocytic alveolitis, but TLR2/9-/- mice had a significant increase in eosinophils in the bronchoalveolar lavage (BAL) fluid compared to WT exposed mice. Th17 cells, but not Th2 cells were detected in the lungs of mice exposed to SR for 14 weeks, suggesting CHP is not associated with a switch to a Th2 type immune response. WT and TLR2/9-/- mice had significantly reduced static compliance compared to WT unexposed mice. To determine whether the restrictive lung defect was associated with fibrosis, we visualized collagen in the lung by histology using Masson's trichrome stain. Measuring collagen staining by positive pixel analysis suggested TLR2/9-/- mice were partially protected from lung fibrosis compared to WT mice. Altogether, the results suggest TLRs 2 and 9 cooperate in neutrophil recruitment and subsequently disease severity and play an integral role in the outcome of HP.
\end{abstract}

\section{Document Type}

Thesis

\section{Degree Name}

Doctor of Philosophy (PhD)

\section{Program}

Biomedical Sciences

Research Advisor

Elizabeth A. Fitzpatrick, Ph.D. 


\section{Keywords}

Chronic hypersensitivity pneumonitis, Hypersensitivity pneumonitis, TLR2, TLR2/9, TLR9, Toll-like receptors.

\section{Subject Categories}

Bacterial Infections and Mycoses | Biological Phenomena, Cell Phenomena, and Immunity | Medical Immunology | Medical Microbiology | Medicine and Health Sciences | Respiratory Tract Diseases

\section{Comments}

Two year embargo expired December 2015 
Toll-like Receptors 2 and 9 Are Important for Innate Immune Cell Activation and Recruitment in Hypersensitivity Pneumonitis

\author{
A Dissertation \\ Presented for \\ The Graduate Studies Council \\ The University of Tennessee \\ Health Science Center \\ In Partial Fulfillment \\ Of the Requirements for the Degree \\ Doctor of Philosophy \\ From The University of Tennessee
}

By

Kelly J. Andrews

December 2013 
Copyright (C) 2013 by Kelly J. Andrews.

All rights reserved. 


\section{DEDICATION}

I would like to dedicate this work to my parents, Thomas and Patricia Andrews, and my sisters, Leslie Slusher and Ashley Andrews,

for encouraging me and supporting me while I followed my dreams. 


\section{ACKNOWLEDGEMENTS}

I would like to thank my advisor, Dr. Elizabeth Fitzpatrick, and my committee members Dr. Maureen McGargill, Dr. Ed Rosloniec, Dr. Chris Waters, and Dr. Ae-Kyung Yi for their guidance and support. I would like to thank previous lab members, Dr. Stephanie Nance for establishing the TLR $2 / 9^{-/-}$mouse colony, and Dr. Hossam Abdelsamed for his invaluable assistance in mouse experiments. I would also like to thank members of Scott Sinclair and Chris Waters' lab, Dr. Manik Gosh, Dr. Andy Schwingshackl, Gabe Rapalo, and Charleen Luellen, for performing mouse lung physiology measurements. In addition, I would like to thank Dr. Thirumala-Devi Kanneganti for the TLR $7^{-1-}$ mouse bone marrow.

I would like to thank members of the Molecular Resources Center, Dr. Bill Taylor, Felicia Waller, and Caitlin Costelle, for their expert help in RNA sequencing, and Ashutosh Pandey who went above and beyond to troubleshoot and finally align the sequences to the mouse genome. I would also like to acknowledge the work of special histology services, especially Linda White, for performing tissue processing and staining of my lung sections, and Ashley Ezekiel for scanning the slides for analysis. This work was supported by the National Institutes of Health (HL084172). 


\begin{abstract}
Hypersensitivity pneumonitis (HP) is an interstitial lung disease caused by repeated inhalation of environmental antigens. The disease is characterized by alveolitis and granuloma formation; however, some patients develop chronic HP (CHP), a restrictive lung disease characterized by fibrosis. Previous studies revealed that neutrophils are recruited into the lung via a MyD88-dependent pathway and regulate disease severity through cytokine production. Toll-like receptors (TLRs) 2 and 9 recognize conserved molecular patterns present in bacteria, and signal through the adaptor molecule MyD88. The goal of my project is to investigate the role of TLRs 2 and 9 in the pathogenesis of HP during the acute phase, granulomatous phase, and chronic phase of the disease. Using the $S$. rectivirgula (SR) animal model of $\mathrm{HP}$, our studies indicated that individually, TLRs 2 and 9 contributed to neutrophil recruitment. We generated TLR2/9 double knockout (TLR2/ $9^{-/}$) mice to determine the extent to which TLRs 2 and 9 cooperate in neutrophil recruitment during HP.
\end{abstract}

During the acute response, TLR2 $/ 9^{-/-}$mice exposed to SR demonstrated a significant reduction in neutrophil recruitment after a single exposure to SR compared to C57BL/6 mice (WT). The reduction in neutrophils was associated with a reduction in the expression of the neutrophil chemokine CXCL2 and inflammatory cytokines TNF $\alpha$ and IL-6 in the BALF. Disease severity in HP is associated with granuloma formation and a Th17 response. After 3 weeks exposure to SR, our results demonstrate a decrease in IL-17 and IL-22 mRNA expression and a corresponding decrease in the percentage of Th17 cells in TLR2 $/ 9^{-/-}$mice compared to WT and single knockout (SKO) mice. The decrease in Th17 cells in TLR2/9 $9^{-/-}$mice was not associated with a significant increase in $\mathrm{T}$ regulatory (Treg) cells or a switch to a Th1 response. In addition, TLR2 ${ }^{-/-}$and TLR $2 / 9^{-/-}$mice have significantly fewer activated $\mathrm{CD}^{+}$Th cells. Interstitial macrophages from TLR2 $/ 9^{-/-}$mice have decreased costimulatory molecule and MHCII expression suggesting they are deficient in T cell activation. However, TLR2 ${ }^{-/-}$, TLR ${ }^{-/-}$, and TLR $2 / 9^{-/-}$still form granulomas in the lung.

To determine the extent to which TLRs 2 and 9 contributed to the development of CHP, WT and TLR2 $/ 9^{-/-}$mice were intranasally exposed to SR three times/week for 15 weeks. WT and TLR2 $/ 9^{-/-}$mice developed a neutrophilic and lymphocytic alveolitis, but TLR $2 / 9^{-/-}$mice had a significant increase in eosinophils in the bronchoalveolar lavage (BAL) fluid compared to WT exposed mice. Th17 cells, but not Th2 cells were detected in the lungs of mice exposed to SR for 14 weeks, suggesting CHP is not associated with a switch to a Th2 type immune response. WT and TLR $2 / 9^{-1-}$ mice had significantly reduced static compliance compared to WT unexposed mice. To determine whether the restrictive lung defect was associated with fibrosis, we visualized collagen in the lung by histology using Masson's trichrome stain. Measuring collagen staining by positive pixel analysis suggested TLR $2 / 9^{-/-}$mice were partially protected from lung fibrosis compared to WT mice. Altogether, the results suggest TLRs 2 and 9 cooperate in neutrophil recruitment and subsequently disease severity and play an integral role in the outcome of HP. 


\section{TABLE OF CONTENTS}

CHAPTER 1. HYPERSENSITIVITY PNEUMONITIS ..................................................1

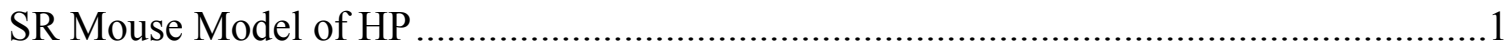

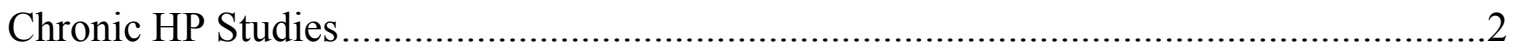

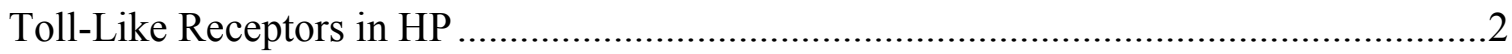

CHAPTER 2. METHODOLOGY ...................................................................................5

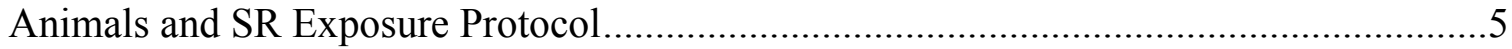

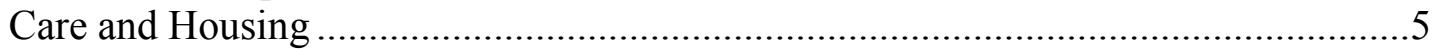

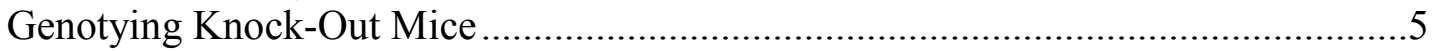

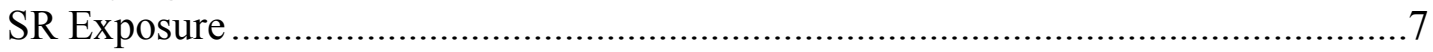

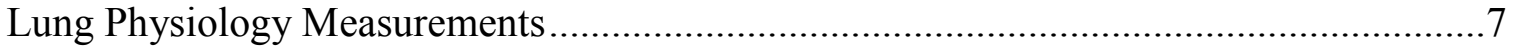

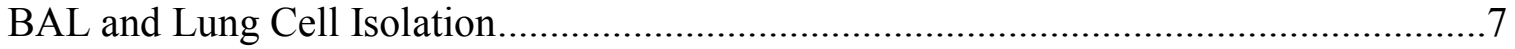

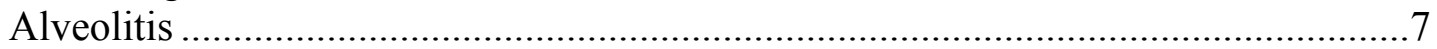

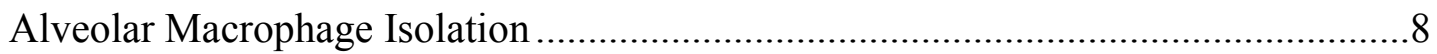

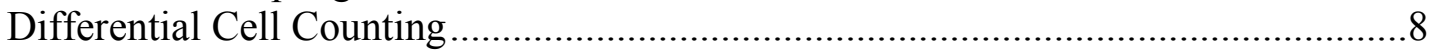

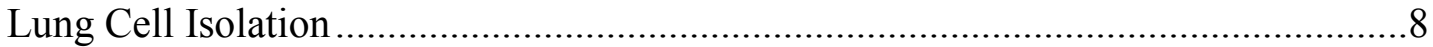

Flow Cytometry

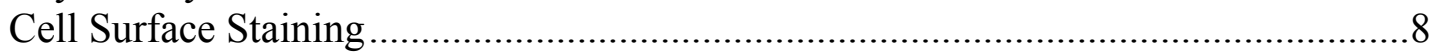

Intracellular Cytokine Staining …..................................................................... 10

Spleen Cell Isolation, Splenic Adherent Cell Enrichment, and Stimulation .....................10

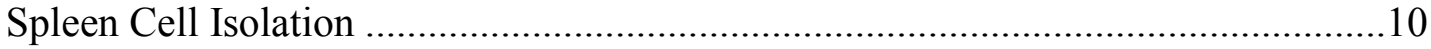

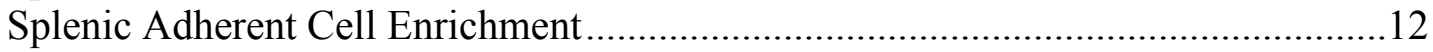

Spleen and Splenic Adherent Cell Stimulation ......................................................... 12

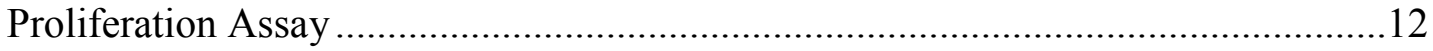

Bone Marrow Derived Macrophage (BMDM) Generation and Stimulation .....................12

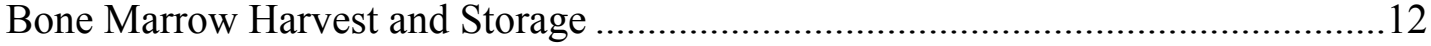

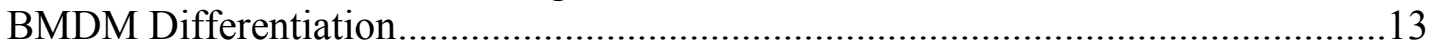

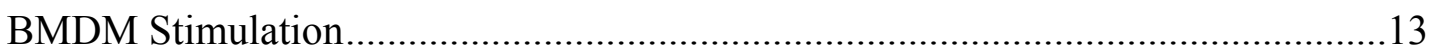

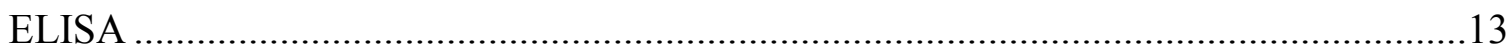

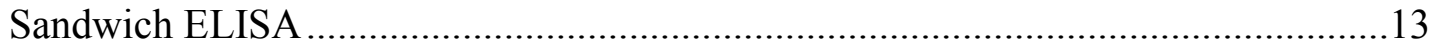

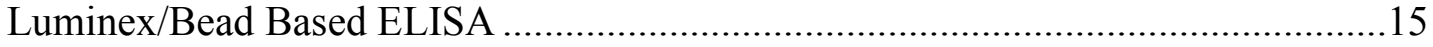

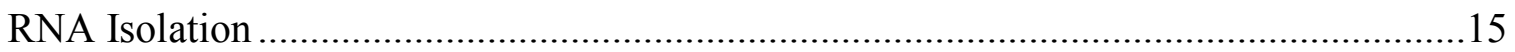

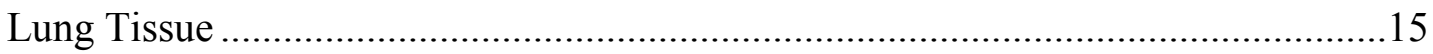

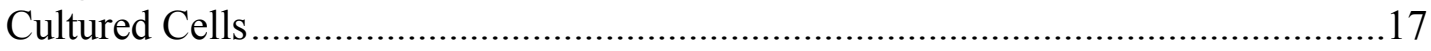

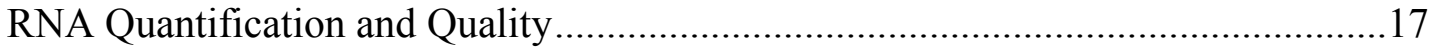

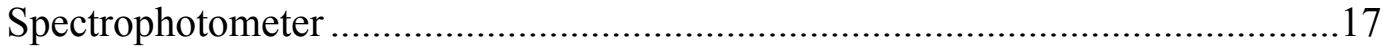

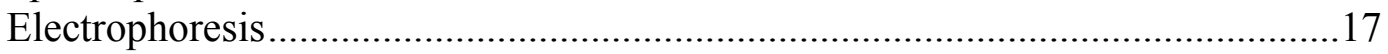

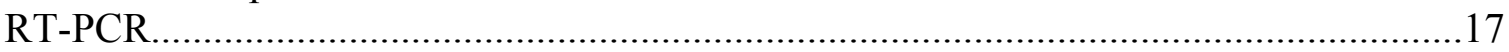

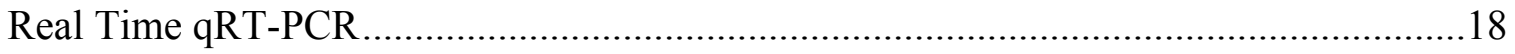

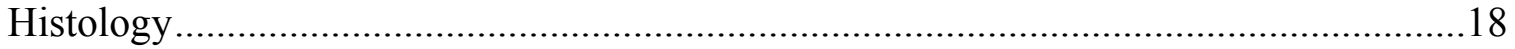

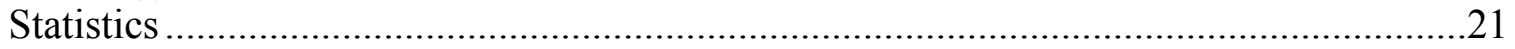




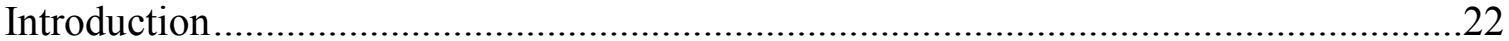

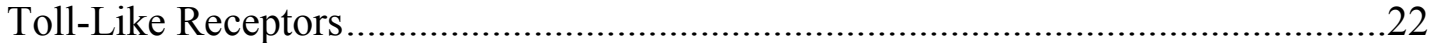

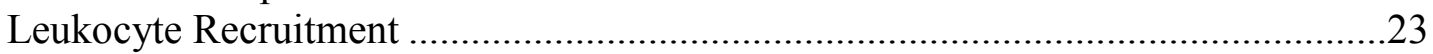

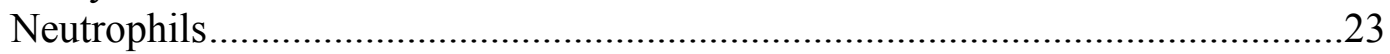

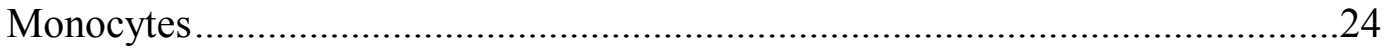

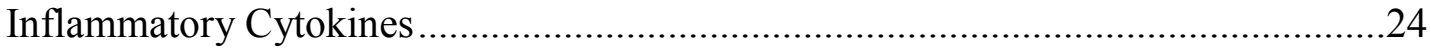

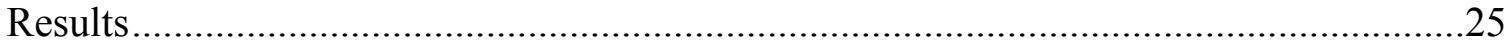

TLRs 2 and 9 Contribute to Neutrophil Chemokine Production and Recruitment into the Airways Following One Exposure to SR In Vivo ....................25

TLR2 $/ 9^{-/-}$Mice Have a Significant Reduction in Inflammatory Cytokine

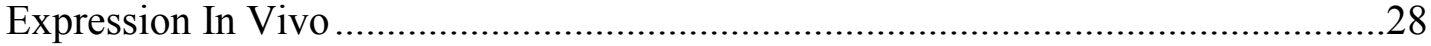

TLR2/9-Dependent and MyD88-Dependent Chemokine and Cytokine Production in SR Stimulated Splenic Adherent Cells In Vitro ..................................33

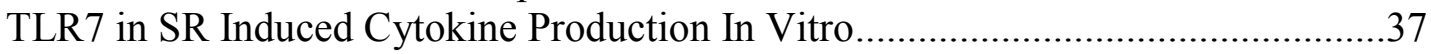

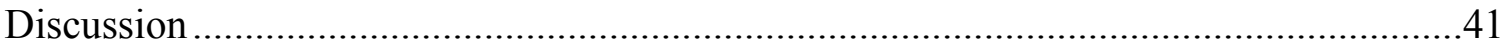

CHAPTER 4. GRANULOMATOUS PHASE....................................................45

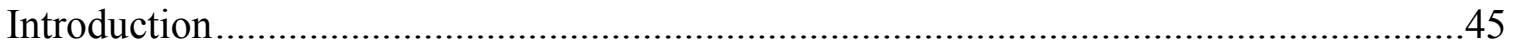

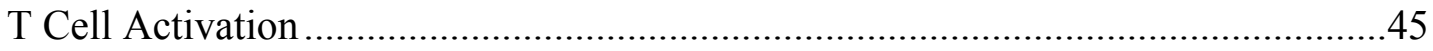

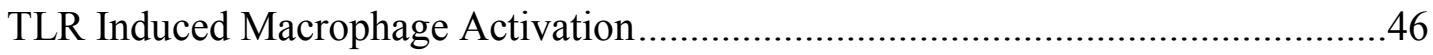

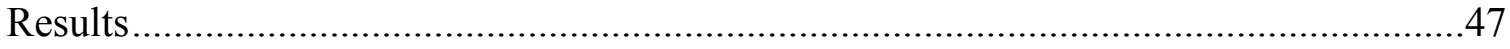

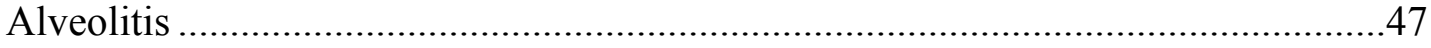

Neutrophil Recruitment and Chemokine Production Are Largely Dependent on TLR2 Following Repeated SR Exposures .....................................47

Lymphocytes Are Recruited to the Airways after 3 Weeks Exposure to SR ........50 TLR2 Is Important for CD4 ${ }^{+}$Th Cell Activation, While TLR9 Is Important for $\mathrm{CD}^{+} \mathrm{T}$ Cell Activation in the Airways .......................................................50

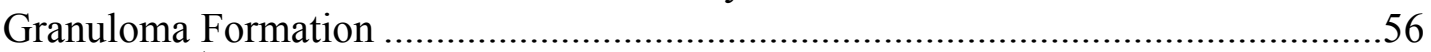

TLR2 $/ 9^{-/-}$Mice Have a Significant Reduction in the Percentage of Activated $\mathrm{T}$ and $\mathrm{B}$ Cells in the Lung ..............................................................56

TLR2 Is the Dominant Receptor for Cytokine Production Following Repeated SR Exposures .............................................................................60

TLR2 $/ 9^{-/-}$Mice Have a Reduced Percentage of Th17 Cells in the Lung.............60

Signaling Through TLR2 Is Important in B Cell Activation ................................ 71

TLRs 2 and 9 Cooperate in Costimulatory Molecule Upregulation on Interstitial Macrophages...............................................................................71

Signaling Through TLR2 Inhibits Costimulatory Molecule Upregulation on Alveolar Macrophages (AMs) …............................................................ 75

TLRs 2 and 9 Are Not Required for Granuloma Formation.............................85

Discussion

CHAPTER 5. CHRONIC PHASE ........................................................................91

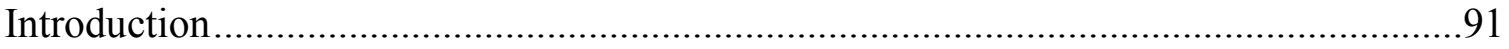

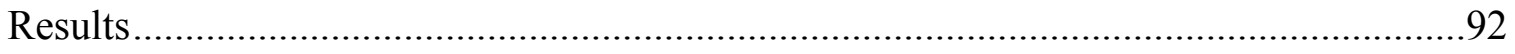


Mice with Long Term Exposure to SR Develop a Neutrophilic and Lymphocytic Alveolitis ...................................................................................92

AMs Have Increased Expression of CD86 and CD206 after Long Term

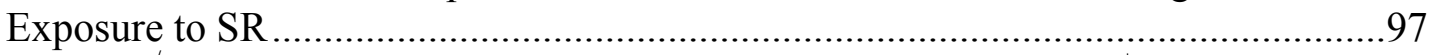

TLR2 $/ 9^{-/-}$Mice Have a Significant Decrease in Activated CD4 ${ }^{+}$Th Cells after Long Term Exposure to SR ........................................................................97

Mice with Long Term Exposure to SR Have Increased Expression of IFN $\gamma$ and IL-17A in the Lung but not IL-4 .................................................................101

$\mathrm{CD}^{+}$Th Cells from Mice with Long Term Exposure to SR Express IL-17A ........103

Mice with CHP Have Decreased Barrier Function and Lung Fibrosis .....................106 Mice with CHP Have Reduced Static Lung Compliance and Total Lung

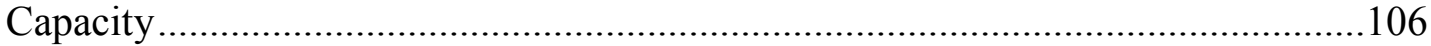

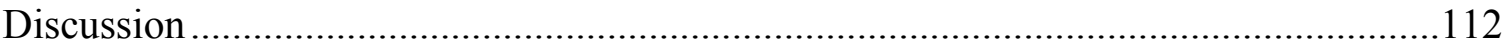

CHAPTER 6. FUTURE DIRECTIONS ...............................................................116

LIST OF REFERENCES .................................................................................................117

APPENDIX. TRANSCRIPTOME ANALYSIS ..................................................132

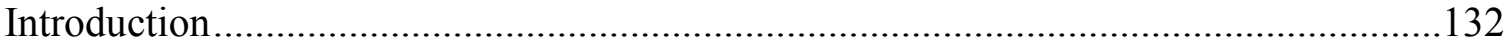

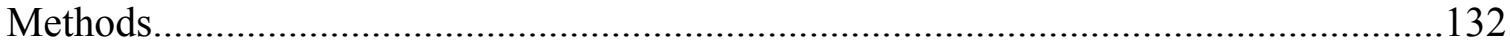

Bone Marrow Harvest and Storage ………………............................................132

Bone Marrow Derived Dendritic Cell (BMDC) Differentiation................................133

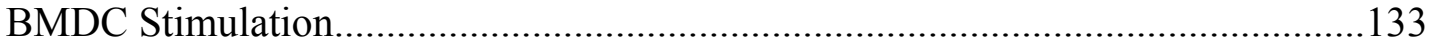

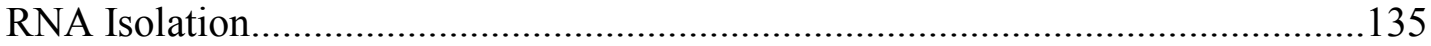

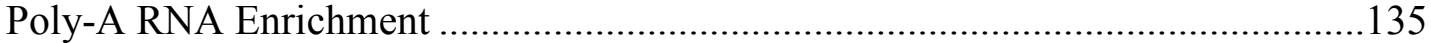

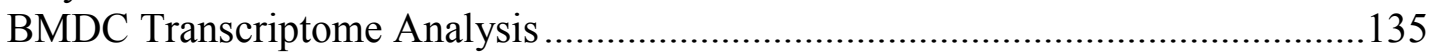

Poly-A RNA Fragmentation and Reverse Transcription ...................................135

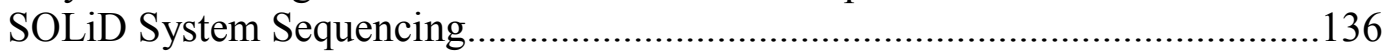

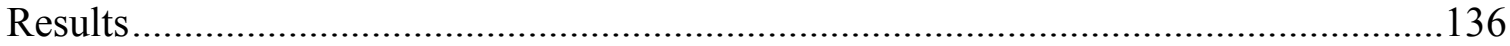

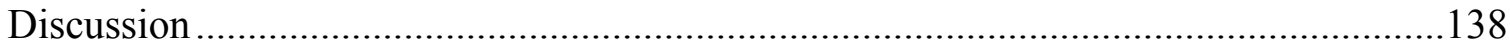

VITA 


\section{LIST OF TABLES}

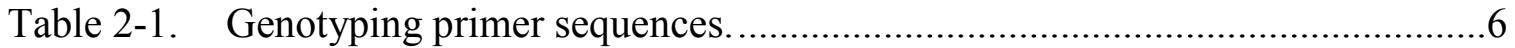

Table 2-2. Genotyping PCR conditions. ...............................................................6

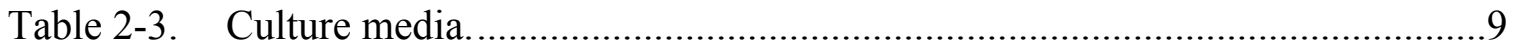

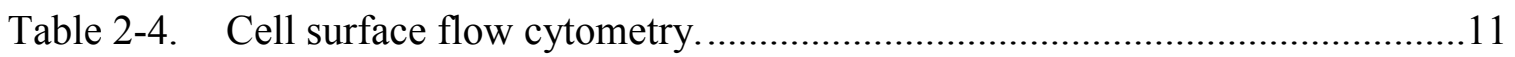

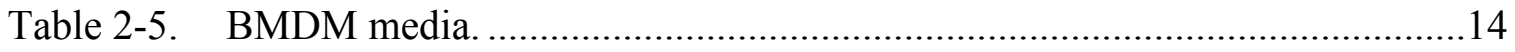

Table 2-6. Enzyme linked immunosorbent assay (ELISA).................................... 16

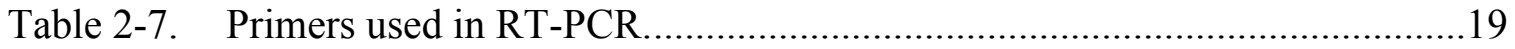

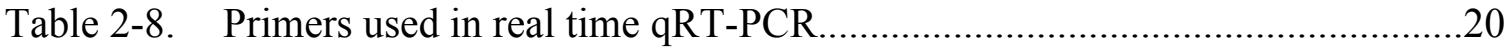

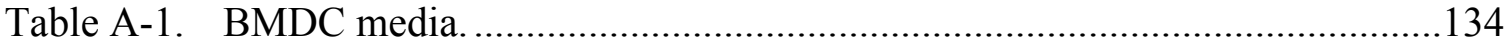

Table A-2. Percentage of genes upregulated $\geq 5 \mathrm{x}$ over WT media that share a biological process.......................................................................... 139

Table A-3. Genes upregulated $\geq 5 \mathrm{x}$ over WT media BMDCs that share a biological function related to an immune response or fibrosis. ................140 


\section{LIST OF FIGURES}

Figure 3-1. Neutrophil recruitment and chemokine expression after one exposure

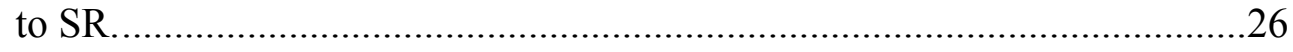

Figure 3-2. Chemokine production after one exposure to SR. ………………………...27

Figure 3-3. Neutrophils are recruited to the peritoneum in response to casein injections in WT and TLR $2 / 9^{-/-}$mice.

Figure 3-4. The decrease in neutrophil recruitment in TLR2/9 ${ }^{-/-}$mice is not due to increased neutrophil apoptosis.................................................................30

Figure 3-5. Inflammatory cytokine expression after one exposure to SR. ......................31

Figure 3-6. Inflammatory cytokine production after one exposure to SR.......................32

Figure 3-7. Chemokine production in splenic adherent cells stimulated with SR in vitro.

Figure 3-8. Cytokine production in splenic adherent cells stimulated with SR in vitro.

Figure 3-9. TLR7 expression is increased in mice exposed to SR for 3 weeks. ..............38

Figure 3-10. SR RNA stimulates cytokine expression in spleen cells in vitro.

Figure 3-11. Bafilomycin inhibits IL-6 and TNF $\alpha$ production in TLR2/9 ${ }^{-/-}$spleen cells stimulated with SR in vitro.

Figure 3-12. Bafilomycin does not inhibit IL-6 and TNF $\alpha$ in BMDM stimulated with SR in vitro.

Figure 4-1. TLR2 is the dominant receptor in neutrophil recruitment to the airways of mice exposed to SR for 3 weeks.

Figure 4-2. Neutrophil chemokine expression is dependent on TLR2 following 3 weeks SR exposure.

Figure 4-3. Mice exposed to SR for 3 weeks have increased leukocyte populations in the BAL.

Figure 4-4. $\mathrm{CD}^{+}$and $\mathrm{CD} 8^{+}$Th cells are recruited to the airways of mice exposed to SR for 3 weeks.

Figure 4-5. Activated $\mathrm{CD}^{+}$and $\mathrm{CD} 8^{+} \mathrm{T}$ cells in the BAL of mice exposed to SR for 3 weeks is reduced in TLR $2 / 9^{-/-}$mice. 
Figure 4-6. TLRs 2 and 9 are not required for activation of B cells, NK cells, or $\gamma \delta$ T cells in the airways of mice exposed to SR for 3 weeks.

Figure 4-7. Reduced activation of $\mathrm{CD} 4^{+}$Th cells in the lungs of TLR2 $2^{-/}$and TLR2 $/ 9^{-/-}$mice exposed to SR for 3 weeks...... .58

Figure 4-8. TLRs 2 and 9 contribute to TNF $\alpha$ expression following repeated SR exposures.

Figure 4-9. TLRs 2 and 9 contribute to Th1- and Th17-associated cytokine production following repeated SR exposures.

Figure 4-10. TLRs 2 and 9 are necessary for the generation of the Th17 response following 3 weeks repeated SR exposure.

Figure 4-11. TLRs 2 and 9 cooperate in sustaining the Th17 response following 3 weeks repeated SR exposure.

Figure 4-12. The reduction in Th17 cells in TLR2/9 $9^{-/}$mice is not due to an increase in $\mathrm{IFN} \gamma^{+}$or $\mathrm{TNF}^{+} \mathrm{Th} 1$ cells.

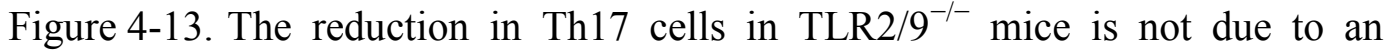
increase in $\mathrm{FoxP}^{+} \mathrm{T}$ regulatory cells.

Figure 4-14. TLR2 is important for the generation of IL-10 expressing $\mathrm{CD}^{+}{ }^{+} \mathrm{T}$ cells.

Figure 4-15. Reduced activation of B cells in the lungs of TLR2/9 ${ }^{-/}$mice.

Figure 4-16. Gating strategy for the identification of interstitial and alveolar macrophages in a lung cell suspension. .76

Figure 4-17. TLRs 2 and 9 cooperate in CD80 and CD86 upregulation on interstitial macrophages after 3 weeks exposure to SR

Figure 4-18. TLR9 is required for MHCII and CD40 upregulation on interstitial macrophages after 3 weeks exposure to SR.

Figure 4-19. TLR2 $/ 9^{-/-}$mice have a significant increase in the percentage of $\mathrm{CD} 11 \mathrm{~b}^{\text {low }}$ AMs after 3 weeks exposure to SR.

Figure 4-20. Loss of TLR2 leads to upregulation of CD80 and MHCII on AMs after 3 weeks exposure to SR.

Figure 4-21. Loss of TLR2 leads to downregulation of CD80 on CD11b ${ }^{\text {high }}$ AMs after 3 weeks exposure to SR.

Figure 4-22. Granuloma development is not dependent on TLRs 2 and 9. .86 
Figure 5-1. Composition of alveolitis in mice exposed to SR for 15 weeks.

Figure 5-2. Th17-associated cytokine expression detected in the lungs of mice exposed to SR for 15 weeks.

Figure 5-3. Changes in BAL composition between 4 week and 14 week exposed mice.

Figure 5-4. TLR2/9 ${ }^{-/-}$mice have a significant decrease in CD11b ${ }^{\text {high }}$ AMs after long term exposure to SR.

Figure 5-5. WT AMs have a significant increase in the expression of CD86 and CD206 expression after 14 weeks SR exposure.

Figure 5-6. TLR2/9 ${ }^{-/}$CD11 $b^{\text {high }}$ AMs have increased expression of CD86 and WT CD11b ${ }^{\text {high }}$ AMs have increase expression of CD206 after 14 weeks exposure to SR.

Figure 5-7. $\mathrm{CD}^{+}$and $\mathrm{CD} 8^{+} \mathrm{T}$ cells in mice exposed to SR 4 and 14 weeks.

Figure 5-8. $\mathrm{CD}^{+}$Th cells in TLR2/9 ${ }^{-/-}$mice express IL-17 but not IL-4 after long term exposure to SR.

Figure 5-9. $\mathrm{CD}^{-} \mathrm{T}$ cells do not have a significant increase in IL-17 or IL-4 expression after long term exposure to SR.

Figure 5-10. Macrophages contribute to IL-17 expression in TLR2/9 ${ }^{-/-}$mice after long term exposure to SR.

Figure 5-11. Evidence of plasma protein leakage across the lung epithelium in the BALF of mice exposed to SR for 4 and 14 weeks.

Figure 5-12. WT mice have a significant increase in lung fibrosis. 110

Figure 5-13. FlexiVent ${ }^{\mathrm{TM}}$ snap shot and pressure volume curve perturbations.

Figure 5-14. WT and TLR2/9 ${ }^{-/-}$mice have a significant decrease in lung compliance.

Figure A-1. Changes in gene expression in WT, MyD88 ${ }^{-/-}, \mathrm{TLR}_{2} / 9^{-/-}, \mathrm{TLR}^{-/-}$, $\mathrm{NOD}^{-/-}$BMDCs stimulated with SR in vitro. 


\section{LIST OF ABBREVIATIONS}

\begin{tabular}{|c|c|}
\hline$\beta \mathrm{TcR}$ & $\beta$ T cell receptor \\
\hline A & Total lung capacity \\
\hline AM & Alveolar macrophage \\
\hline ANOVA & Analysis of variance \\
\hline $\mathrm{APC}(\mathrm{s})$ & Antigen presenting cell(s) \\
\hline BAL & Bronchoalveolar lavage \\
\hline BALF & Bronchoalveolar lavage fluid \\
\hline BMDC & Bone marrow derived dendritic cell \\
\hline BMDM & Bone marrow derived macrophage \\
\hline $\mathrm{C}$ & Compliance \\
\hline CHP & Chronic hypersensitivity pneumonitis \\
\hline Cst & Static compliance \\
\hline $\mathrm{DC}$ & Dendritic cell \\
\hline $\mathrm{E}$ & Elastance \\
\hline Est & Static elastance \\
\hline HP & Hypersensitivity pneumonitis \\
\hline $\mathrm{IM}$ & Interstitial macrophage \\
\hline $\mathrm{KO}$ & Knock-out \\
\hline MHCII & Major histocompatibility complex class II \\
\hline $\mathrm{MyD} 88^{-/-}$ & MyD88 knock-out \\
\hline PRRs & Pattern recognition receptors \\
\hline $\mathrm{R}$ & Resistance \\
\hline
\end{tabular}




$\begin{array}{ll}\text { RPKM } & \text { Reads per kilobase of exon model per million mapped reads } \\ \text { SKO } & \text { Single knock-out } \\ \text { SR } & \text { Saccharopolyspora rectivirgula } \\ \text { Th } & \text { T helper cell } \\ \text { TLR2 }{ }^{-/-} & \text {TLR2 knock-out } \\ \text { TLR2/9 }{ }^{-/-} & \text {TLR2 \& TLR9 double knock-out } \\ \text { TLR9 }{ }^{-/-} & \text {TLR9 knock-out } \\ \text { TLR(s) } & \text { Toll-like receptor(s) } \\ \text { Treg } & \text { T regulatory cell } \\ \text { WT } & \text { Wild type }\end{array}$




\section{CHAPTER 1. HYPERSENSITIVITY PNEUMONITIS}

Hypersensitivity Pneumonitis (HP), or extrinsic allergic alveolitis, develops following repeated exposure to a wide variety of inhaled environmental antigens (reviewed in Kurup et al., 2006). The environmental antigens that induce HP include organic dusts, animal proteins, fungi, and bacteria. The different types of HP are frequently named after the occupation or activity that results in exposure to the inciting agent (reviewed in Selman et al., 2012). Farmer's Lung disease is one of the most common types of HP and is caused by repeated inhalation of the thermophilic gram positive bacteria Saccharopolyspora rectivirgula (SR), which grows in moldy hay.

Hypersensitivity pneumonitis can be classified into three subtypes: acute, subacute, and chronic. Acute HP is characterized by flu-like symptoms such as fever, sweating, myalgia, aching joints, headaches, accompanied by cough, breathlessness, dyspnea, wheeze, and chest tightness a few hours after exposure, and the disease can be intermittent or progressive (reviewed in Ismail et al., 2006; Kurup et al., 2006; Selman et al., 2012). The subacute stage often occurs after prolonged low dose exposure over weeks or months as patients develop cough, dyspnea, and hypoxemia after exertion with mild or absent acute symptoms (reviewed in Ismail et al., 2006; Kurup et al., 2006; Selman et al., 2012). In the lung, patients develop a neutrophilic and lymphocytic alveolitis, interstitial bronchiolocentric pneumonitis, and small loosely formed noncaseating granulomas (Selman et al., 2010). In some patients, the disease can progress to chronic HP (CHP), however some patients may develop CHP without ever experiencing acute symptoms. Chronic symptoms are similar to subacute HP, but in addition CHP is associated with emphysema and a restrictive lung disease characterized by fibrosis and reduced lung compliance (Costabel et al., 2012; Erkinjuntti-Pekkanen et al., 1998; Selman et al., 2012). CHP is progressive and irreversible and is associated with high morbidity and mortality (Hanak et al., 2008; Vourlekis et al., 2004).

The best treatment option for HP is antigen avoidance if the antigen can be identified. If exposure can be stopped, patients with acute and subacute HP will recover. In chronic HP, antigen identification and avoidance will not completely resolve symptoms but is associated with reduced mortality (Fernandez Perez et al., 2013). Corticosteroids are also often prescribed, but have little effect on disease outcomes (Kokkarinen et al., 1992). Deaths where HP is the underlying or contributing cause have steadily increased from 1979 to 2007 (NIOSH., 2012). Therefore, more research into how $\mathrm{HP}$ progresses to CHP is needed to develop new treatment options.

\section{SR Mouse Model of HP}

The SR mouse model of Farmer's Lung disease has identified many of the pathogenic mechanisms that lead to disease. Mice intranasally inoculated with SR 3 days/week for 3 weeks develop a neutrophilic alveolitis that becomes more lymphocytic

as exposures continue (Denis, 1992; Denis et al., 1992). The influx of neutrophils is 
accompanied by a cytokine response consisting of TNF $\alpha$, IL-6, IL-17, IFN $\gamma$, and CXCL2/MIP-2, macrophage and dendritic cell activation, and lymphocyte recruitment. Previous studies have revealed HP is a T cell mediated disease. Nude mice, which lack a thymus and do not develop mature T cells, do not develop HP (Ohta et al., 1993), and $\mathrm{TcR} \beta^{-1-}$ mice, which lack $\mathrm{CD} 4^{+}$and $\mathrm{CD} 8^{+} \mathrm{T}$ cells, do not form granulomas or develop severe fibrosis in response to SR (Simonian et al., 2009). In addition, the adoptive transfer of $\mathrm{CD}^{+} \mathrm{T}$ cells or ex vivo expanded Th1 cells from exposed mice into naïve mice confers disease upon antigen exposure (Schuyler et al., 1997; Schuyler et al., 1993). Granuloma formation in HP is dependent on $\mathrm{T}$ cells as well as the cytokine IFN $\gamma$, produced either from $\mathrm{T}$ cells or innate immune cells (Gudmundsson and Hunninghake, 1997; Nance et al., 2005). However, recent studies have shown IL-17 and the Th17 response is associated with increased disease severity and fibrosis in HP (Joshi et al., 2009; Simonian et al., 2009).

\section{Chronic HP Studies}

Unfortunately, little is known about the transition between HP and CHP. One hypothesis suggests the development of progressive fibrosis in CHP is associated with a switch to a Th2 response. A study which looked at T cells responses in patients with pigeon breeder's disease, another common type of HP, found an increase in $\mathrm{CD}^{+}$and $\mathrm{CD}^{+} \mathrm{T}$ cells expressing IL-4 and a decrease in T cells expressing IFN $\gamma$ in patients with CHP compared to subacute HP (Barrera et al., 2008). This is in agreement with a mouse model of chronic pigeon breeder's disease; the authors found that IL-4 / IL-13 expression and a Th2-biased immune response correlated with increased lung fibrosis (Mitaka et al., 2011). Fibrotic diseases often develop in the presence of Th2-associated cytokines IL-4 and IL-13 (Gharaee-Kermani et al., 2001; Jakubzick et al., 2003; Lee et al., 2001); however, previous studies have shown IL-4 and Th2-biased immune responses are protective in SR induced HP (Butler et al., 2002; Gudmundsson et al., 1998; JimenezAlvarez et al., 2010; Matsuno et al., 2007).

Neutrophils have also been associated with lung fibrosis in CHP. There is a correlation between the level of neutrophils in the lung tissue and severity of fibrosis in patients with CHP (Pardo et al., 2000). The neutrophils expressed high levels of gelatinase B and collagenase 2, which contribute to lung damage. IL-17 expression is associated with the induction of neutrophil chemokines and neutrophil recruitment in the lung (Hellings et al., 2003; Laan et al., 1999; Miyamoto et al., 2003; Witowski et al., 2000). In addition, recent studies of HP in mice have shown that neutrophils and macrophages were the main cell type that expressed IL-17A in HP, and that neutrophil depletion decreased the amount of lung collagen (Hasan et al., 2013).

\section{Toll-Like Receptors in HP}

Because neutrophils are one of the first cell types recruited to the lung after

antigen exposure, and have been implicated in the pathogenesis of CHP, our lab became 
interested in the pattern recognition receptors (PRRs) responsible for SR recognition and neutrophil recruitment. PRRs recognize conserved molecular patterns present on bacteria, viruses, and other pathogens and are important in innate immune cell activation and phagocytosis. Toll-like receptors (TLRs) are a family of PRRs expressed either on the cell surface or in endosomes, and most signal through the adaptor molecule myeloid differentiation primary response gene 88 (MyD88). Previous studies in our lab demonstrated that MyD88-dependent receptors are necessary for the initial production of pro-inflammatory cytokines, neutrophil chemokines, and subsequently neutrophil recruitment following acute SR exposure (Nance et al., 2008). One of the MyD88dependent TLRs involved in recognition of gram positive bacteria is TLR2, and our previous studies revealed that SR interacts with TLR2 and can activate the TLR2 signaling pathway that leads to NF- $\mathrm{kB}$ activation using a luciferase reporter assay in vitro. TLR2 forms heterodimers with either TLR1 or TLR6, and previous studies have shown TLR6 is involved in SR induced immune response in HP (Fong et al., 2010). Mice deficient in TLR2 have a significant reduction in the neutrophil chemokine CXCL2/MIP-2 but not neutrophil recruitment. Therefore, additional MyD88-dependent receptors must be involved in SR recognition and neutrophil recruitment.

Previous studies have shown the importance of TLR9 signaling in other gram positive organisms such as Mycobacterium tuberculosis, and Mycobacterium avium, (Bafica et al., 2005; Carvalho et al., 2011; Daito et al., 2011), fungi such as Aspergillus fumigates and Cryptococcus neoformans and Stachybotrys chartarum (Bhan et al., 2011; Ramaprakash et al., 2009; Zhang et al., 2010), parasites such as Trypanosoma cruzi (Bafica et al., 2006), and viruses such as Herpes simplex virus (Sorensen et al., 2008). In addition, TLR2 can cooperate with TLR9 to induce protective immunity as mice deficient in both TLRs 2 and 9 resulted in increased pathogen burden, and reduced cytokine production, particularly IL-6, TNF $\alpha$, and IL-12p40 (Bafica et al., 2006; Bafica et al., 2005; Sorensen et al., 2008). SR is a gram positive bacteria, and exposure induces IL-6, TNF $\alpha$, and IL-12p40 (Schuyler et al., 2000), and therefore TLR9, along with TLR2, may contribute to the immune response to SR.

The goal of my project is to investigate the role of TLRs 2 and 9 in the pathogenesis of HP during the acute phase, the granulomatous phase, and the chronic phase. We hypothesize mice doubly deficient in TLRs 2 and 9 will have reduced production of neutrophil chemokines and inflammatory cytokines TNF $\alpha$, IL-6, and IL-12p40 resulting in reduced neutrophil recruitment during the acute innate immune response. During the granulomatous phase, we hypothesize the loss of TLRs 2 and 9 in SR expose mice will also result in reduced neutrophil recruitment as well as reduced antigen presenting cell activation and upregulation of costimulatory molecules and MHCII necessary for the activation of naive $\mathrm{T}$ cells; and therefore $\mathrm{CD}^{+}{ }^{+} \mathrm{Th}$ cell activation during the adaptive immune response will be reduced. The reduced $\mathrm{CD} 4^{+} \mathrm{Th}$ cell activation, combined with the reduction in inflammatory cytokines IL-6 and IL-12p40 (the common chain for IL-12 and IL-23) will result in reduced polarization of Th1 and Th17 cells in TLR2/ $9^{-/-}$mice. Granuloma formation depends on physical signals as well as cytokine signals, and the reduced interstitial macrophage and $\mathrm{CD}^{+} \mathrm{Th}$ cell activation and inflammatory cytokine production in TLR $2 / 9^{-/-}$mice will result in reduced 
granuloma formation. Finally, the reduced neutrophil recruitment and Th17 cell response in TLR2 $/ 9^{-/}$mice will result in reduced fibrosis during CHP and protect against the development of restrictive lung disease.

To test these hypotheses, we will use an animal model of HP where WT, TLR2 ${ }^{-/-}$, TLR9 ${ }^{-/}$, and TLR2 $/ 9^{-1-}$ mice will be exposed to SR once for experiments during the acute phase, 3 times/week for 3 weeks for experiments during the granulomatous phase, and 3 times/week for 14 or 15 weeks for experiments during the chronic phase. Changes in neutrophils and other immune cell populations recruited to the airways and lung tissue will measured by alveolitis and flow cytometry, and changes in Th17 cell and other Th cell populations will be evaluated by intracellular flow cytometry between WT and KO mice. At each time point, SR induced cytokine and chemokine production will be analyzed for reductions in expression and protein levels in KO mice compared to WT SR exposed mice. The development of granulomas during the granulomatous phase, and fibrosis, and reduced lung function during the chronic phase will be assessed in WT and $\mathrm{KO}$ mice as a measure of disease severity. Data will be analyzed by statistical software to compare differences between KO and WT SR exposed mice to assess statistical significance. 


\title{
CHAPTER 2. METHODOLOGY
}

\author{
Animals and SR Exposure Protocol
}

\section{Care and Housing}

C57BL/6 mice (WT) were purchased from Jackson Laboratories (Bar Harbor, $\mathrm{ME})$ at 6 weeks of age. MyD88 ${ }^{-/-}, \mathrm{TLR}^{-/-}, \mathrm{TLR}^{-/-}$, and TLR2 $/ 9^{-/-}$mice on a C57BL/6 background were bred at UTHSC and were in at least the $5^{\text {th }}$ generation backcross.

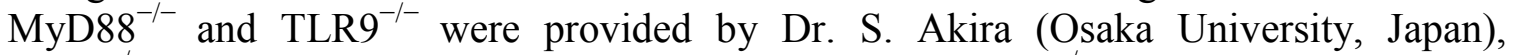
$\mathrm{TLR}^{-/-}$mice were purchased from Jackson Lab, and TLR $2 / 9^{-/-}$mice were generated by breeding TLR2 ${ }^{-/-}$and TLR9 ${ }^{-/-}$mice. All animals were housed in sterile micro-isolator cages with sterile food and water ad libitum and were maintained by the Division of Comparative Medicine at the University of Tennessee Health Science Center according to the guidelines of the Animal Welfare Act. All animal care procedures were performed according to protocols approved by the UTHSC animal care and use committee.

\section{Genotyping Knock-Out Mice}

The genotype of knock-out mice was confirmed by PCR. A tail snip was collected from mouse pups and digested in digestion buffer $(500 \mathrm{mM} \mathrm{KCl}, 100 \mathrm{mM}$ Tris, $1 \% \mathrm{NP}-40$, $1 \%$ Tween, $200 \mu \mathrm{g} / \mathrm{mL}$ proteinase $\mathrm{K}$ ) overnight at $55^{\circ} \mathrm{C}$ with shaking. The enzyme was heat inactivated at $100^{\circ} \mathrm{C}$ for 5 minutes then centrifuged at $8500 \mathrm{rpm}$ (Centrifuge 5451D, Eppindorf, Hamburg, Germany) for 5 minutes. The supernatant containing DNA was transferred to a clean tube. The DNA concentration of supernatant was determined using an Ultrospec 2000 spectrophotometer (Pharmacia Biotech, Piscataway, NJ). A portion of the supernatant $(2 \mu \mathrm{L})$ was diluted 1:30 in nuclease free water and the absorbance at $260 \mathrm{~nm}$ and $280 \mathrm{~nm}$ was measured. The concentration of DNA $(\mu \mathrm{g} / \mathrm{mL})$ is equal to the absorbance at $260 \mathrm{~nm}$ multiplied by the dilution factor and $50 \mu \mathrm{g} / \mathrm{mL}$. The supernatant containing DNA was diluted to a concentration of $200 \mu \mathrm{g} / \mathrm{mL}$ for use in PCR.

DNA (200ng) was added to a PCR master mix (Go Taq PCR core system I, Promega, Fitchburg, WI) containing Taq polymerase, nucleotides, gene specific forward and reverse primers (Table $\mathbf{2 - 1}$ ), and $\mathrm{MgCl}_{2}$ in a reaction buffer. The PCR reaction was carried out in a PCR thermocycler (Mastercycler epgradient S, Eppindorf, Hamburg, Germany). Each reaction began with a denaturation step of $94^{\circ} \mathrm{C}$ followed by an annealing step at a primer specific temperature and an elongation step at $72^{\circ} \mathrm{C}$ repeated for 35 or 36 cycles (Table 2-2). 
Table 2-1. Genotyping primer sequences.

\begin{tabular}{lllll}
\hline Gene & Forward primer & Reverse primer & Neomycin primer & Product \\
\hline TLR2 & CTT CCT GAA & ACG AGC AAG & GGG CCA GCT & WT: $498 \mathrm{bp}$ \\
& TTT GTC CAG & ATC AAC AGG & CAT TCC TCC & KO: $334 \mathrm{bp}$ \\
& TAC A & AGA & CAC & \\
TLR9 & GCT CAT TTA & AGG TTC TGG & CGT CGT GAC & WT: $377 \mathrm{bp}$ \\
& GCC CTG GCT & GCT CAA TGG & CCA TGG CGA & KO: $662 \mathrm{bp}$ \\
& CTC ACA G & TCA TGT & TG & \\
MyD88 & TGG CAT GCC & GTC AGA AAC & ATC GCC TTC & WT: $563 \mathrm{bp}$ \\
& TCC ATC ATA & AAC CAC CAC & TAT CGC CTT & KO: $625 \mathrm{bp}$ \\
& GTT AAC C & CAT GC & CTT GAC G & \\
\hline
\end{tabular}

Table 2-2. Genotyping PCR conditions.

\begin{tabular}{lccccccccc}
\hline Gene & \multicolumn{2}{c}{ Denaturation } & & \multicolumn{2}{c}{ Annealing } & & \multicolumn{2}{c}{ Elongation } & Cycles \\
\cline { 2 - 3 } & Temp & Time & & Temp & Time & & Temp & Time & \\
\hline TLR2 & $94^{\circ} \mathrm{C}$ & $45 \mathrm{~s}$ & & $63^{\circ} \mathrm{C}$ & $45 \mathrm{~s}$ & & $72^{\circ} \mathrm{C}$ & $60 \mathrm{~s}$ & 36 \\
TLR9 & $94^{\circ} \mathrm{C}$ & $45 \mathrm{~s}$ & & $60^{\circ} \mathrm{C}$ & $60 \mathrm{~s}$ & & $72^{\circ} \mathrm{C}$ & $60 \mathrm{~s}$ & 36 \\
MyD88 & $94^{\circ} \mathrm{C}$ & $45 \mathrm{~s}$ & & $65^{\circ} \mathrm{C}$ & $60 \mathrm{~s}$ & & $72^{\circ} \mathrm{C}$ & $60 \mathrm{~s}$ & 35 \\
\hline
\end{tabular}




\title{
SR Exposure
}

The $S$. rectivirgula (SR) (strain designation A1313 - ATCC) was grown at $55^{\circ} \mathrm{C}$ in trypticase soy broth. The bacterial preparation was washed in endotoxin free distilled water 3 times followed by lyophilization. The lyophilized preparation was reconstituted with endotoxin free saline. Mice were anesthetized with isoflurane and mice inoculated intranasally with $50 \mu \mathrm{g}$ SR in $40 \mu \mathrm{L}$ sterile saline. For chronic exposure experiments, the SR dose was increased to $150 \mu \mathrm{g}$ SR in $50 \mu \mathrm{L}$ sterile saline. In acute experiments, mice were exposed once. For experiments during the granulomatous phase, mice were exposed three times/week for 3 weeks. For experiments during the chronic phase, mice were exposed three times/week for 13 weeks and once on week 14 or three times/week for 14 weeks and once on week 15.

\section{Lung Physiology Measurements}

Mice were weighed then anesthetized with intraperitoneal ketamine/xylazine (1:1) $(0.15 \mathrm{~mL} / 10 \mathrm{~g})$. A tracheotomy was performed using an 18-Ga needle. Adequate anesthesia was maintained with inhalation of $1 \%$ isoflurane. Temperature was maintained with a heat lamp. On the flexiVent ${ }^{\mathrm{TM}}$ mechanical ventilator (SCIREQ Scientific Respiratory Equipment Inc, Montreal, QC), ventilation was $10 \mathrm{~mL} / \mathrm{kg}$, and respiratory rate was kept at 150 breaths/min. Positive end-expiratory pressure (PEEP) was $2 \mathrm{cmH}_{2} \mathrm{O}$. Mice were inflated to total lung capacity (TLC) to open airways before taking measurements. A snapshot 150 perturbation inflated lungs in a sinusoidal forced oscillation waveform to measure dynamic lung compliance (C), elastance (E), and airway resistance (R). A pressure volume curve was generated by a step-wise inflation of the lung to total lung capacity and deflation back to functional residual capacity. The flexivent software preformed a curve fit of the data to the Salazar-Knowles equation to determine static compliance (Cst), static elastance (Est) and total lung capacity (A).

\section{BAL and Lung Cell Isolation}

\begin{abstract}
Alveolitis
Bronchoalveolar lavage (BAL) was performed by intratracheal injection of 1.0-1.5mL PBS or 2mM PBS-EDTA into the lungs with immediate vacuum aspiration. The amount of fluid recovered was routinely around $70 \%$. Cells were recovered from the BAL fluid by centrifugation (Sorvall Legend RT, Thermo Fisher Scientific, Waltham, MA) at 1500rpm for 5 minutes and live cells counted using trypan blue dye exclusion on a hemocytometer. The BAL fluid was aliquoted and frozen at $-80^{\circ} \mathrm{C}$ until used in ELISA assays for cytokine and chemokine measurement.
\end{abstract}




\section{Alveolar Macrophage Isolation}

To isolate alveolar macrophages, the lungs were exhaustively flushed with 14-15mL 2mM PBS-EDTA injected intratracheally. Cells were then recovered by centrifugation (Sorvall Legend RT, Thermo Fisher Scientific, Waltham, MA) at 1500rpm for 5 minutes for use in flow cytometry.

\section{Differential Cell Counting}

In some experiments, an aliquot of BAL cells were centrifuged onto a slide for differential cell counting. Slides were heat fixed and stained with a modified Wright Giemsa stain (HEMA 3 stat pack, Fisher Scientific) according to manufacturer's instructions. Slides were digitized (Scanscope XT, Aperio, Vista, CA) and analyzed using Spectrum Imagescope software (Aperio). Cells in a minimum of 4 fields at 20x were analyzed until a minimum of 200 cells were counted and identified by nuclear shape and cytoplasmic staining. Multinucleated macrophages were identified as macrophages that contained two or more nuclei.

\section{Lung Cell Isolation}

Lungs were perfused with phosphate-buffered saline (PBS) to remove blood and lobes removed and placed in culture media (Table 2-3). Lung lymphocytes were isolated from the lung in a method similar to Abraham et al. (Abraham et al., 1990). Lung tissue was digested with collagenase $(20 \mathrm{U} / \mathrm{mL})$ and deoxyribonuclease I $(40 \mu \mathrm{g} / \mathrm{mL})$ for 45 minutes at $37^{\circ} \mathrm{C}$. Cells were freed by disruption in a Stomacher 80 tissue processor (Seward, West Sussex, UK) and then isolated by centrifugation on a discontinuous percoll (Sigma, St. Louis, MO) gradient. Cells were underlaid with a $40 \%$ percoll solution, followed by an $80 \%$ percoll solution and centrifuged at 2000rpm for 20 minutes and stopped with a light brake. Mononuclear cells were isolated at the $40 / 80 \%$ interface and used in flow cytometry and ex vivo cell stimulations.

\section{Flow Cytometry}

\section{Cell Surface Staining}

Flow cytometry was performed on isolated BAL and lung cells. Cells were suspended in FACS buffer (2-3\% heat inactivated FCS in PBS, in some experiments also containing $2 \mathrm{mM}$ EDTA). The Fc-receptor was blocked with anti-CD16/32 antibody (BD Biosciences, San Jose, CA). Unbound antibody was washed with FACS buffer and cells were stained with fluorochrome-conjugated antibodies to CD11b, CD11c, Gr-1, F4/80, CD45, CD4, CD8, $\beta$ TcR, $\gamma \delta$ TcR, NK1.1, CD19, CD40, CD80, CD86, MHCII, and 
Table 2-3. Culture media.

\begin{tabular}{lll}
\hline Reagent & \multicolumn{1}{c}{ Manufacturer } & \multicolumn{1}{c}{ Location } \\
\hline RPMI & Cellgro, Mediatech Inc. & Manassas, VA \\
$5 \%$ heat inactivated FCS & Hyclone, Thermo Scientific & Logan, UT \\
$2 \mathrm{mM}$ glutamine & Cellgro, Mediatech Inc. & Manassas, VA \\
$100 \mathrm{IU} / \mathrm{mL}$ penicillin & Cellgro, Mediatech Inc. & Manassas, VA \\
$100 \mu \mathrm{g} / \mathrm{mL}$ streptomycin & Cellgro, Mediatech Inc. & Manassas, VA \\
$1 \mu \mathrm{L} / \mathrm{L} \beta$-mercaptoethanol & Fisher Scientific & Waltham, MA \\
\hline
\end{tabular}


CD206 (BD Biosciences, San Jose, CA; eBiosciences, San Diego, CA; Biolegend, San Diego, CA; or Biosource, Invitrogen, Carlsbad, CA) in a volume of approximately $200 \mu \mathrm{L}$. After a 30 minute incubation in the dark, the cells were washed with FACS buffer twice then resuspended for analysis on a BD LSRII flow cytometer (BD Biosciences, San Jose, CA). The flow cytometer was compensated with fluorochrome-conjugated beads (BD Biosciences, San Jose, CA). Autofluorescence was compensated with autofluorescence ${ }^{-}$spleen cells and autofluorescence ${ }^{+}$unstained lung cells. For most experiments between 30,000 and 50,000 events were collected. Data was analyzed with FACSDiva software (BD Biosciences, San Jose, CA). Cells were identified by expression of surface markers (Table 2-4) and analyzed for the expression of classical or alternative activation makers.

\section{Intracellular Cytokine Staining}

For intracellular cytokine staining, $5 \times 10^{5}$ lung cells were incubated with SR lysate $(4 \mu \mathrm{g})$ or plate bound $\alpha-\mathrm{CD} 3$ for 3 hours with an additional 3 hours in the presence of brefeldin A (Golgi Plug, BD Biosciences, San Jose, CA) or monensin (Golgi Stop, BD Biosceinces, San Jose, CA). The SR lysate was generated using the Y-PER reagent (Pierce) following manufacturer's instructions. In some experiments, cells were stimulated with PMA and ionomycin in the presence of brefeldin A for 5 hours. The cells were incubated with antibodies to $\mathrm{CD} 45, \mathrm{CD} 4$, and $\beta \mathrm{TcR}$ to identify $\mathrm{CD} 4^{+} \mathrm{T}$ cells and then fixed (BD Cytofix, BD Biosciences, San Jose, CA) and permeabilized with $1 \%$ saponin followed by incubation with IL-17A, IFN $\gamma$, TNF, IL-10, or isotype control antibodies. Alternatively, cells were fixed with FoxP3 fixation/permeabilization solution (eBiosciences, San Diego, CA) and stained with FoxP3 and IL-17F or isotype control antibodies. A minimum of 50,000 events/sample was collected on a BD Biosciences LSRII cytometer (BD Biosciences, San Jose, CA). Expression of cell surface markers and intracellular cytokines was analyzed using FACSDiva software (BD Biosciences, San Jose, CA).

\section{Spleen Cell Isolation, Splenic Adherent Cell Enrichment, and Stimulation}

\section{Spleen Cell Isolation}

The spleen was harvested from WT, TLR2 ${ }^{-/-}$, TLR9 ${ }^{-/}$, TLR2 $/ 9^{-/-}$, and MyD88 $8^{-/-}$ mice. Spleen cells were freed by disruption in a Stomacher 80 tissue processor (Seward, West Sussex, UK). RBCs were lysed with RBC lysis buffer, then washed with media and pelleted at 1500rpm for 5 minutes. 
Table 2-4. Cell surface flow cytometry.

\begin{tabular}{|c|c|c|c|}
\hline Cell type & $\begin{array}{c}\text { Identifying surface } \\
\text { markers }\end{array}$ & $\begin{array}{c}\text { Activation } \\
\text { markers }\end{array}$ & $\begin{array}{c}\text { Alternative } \\
\text { activation markers }\end{array}$ \\
\hline Neutrophil & $\begin{array}{l}\mathrm{CD} 45^{+} / \mathrm{CD} 11 \mathrm{~b}^{+} / \\
\mathrm{Gr}^{+} 1^{+}\end{array}$ & & \\
\hline $\begin{array}{l}\text { Interstitial } \\
\text { macrophage }\end{array}$ & $\begin{array}{l}\mathrm{CD} 45^{+} / \mathrm{CD}^{+} 1 \mathrm{~b}^{+} / \\
\mathrm{F} 4 / 80^{+} / \\
\text {autofluorescence }^{-}\end{array}$ & $\begin{array}{l}\mathrm{CD}^{+} 0^{+} / \mathrm{CD} 86^{+} / \\
\mathrm{CD}^{+} 0^{+} / \mathrm{MHCII}^{\mathrm{high}}\end{array}$ & \\
\hline $\begin{array}{l}\text { Alveolar } \\
\text { macrophage }\end{array}$ & $\begin{array}{l}\mathrm{CD} 45^{+} / \\
\mathrm{CD} 11 \mathrm{~b} \text { high/low } / \\
\mathrm{CD} 11 \mathrm{c}^{+} / \mathrm{F} 4 / 80^{+} / \\
\mathrm{CD} 205^{+} / \\
\text {autofluorescence }^{+}\end{array}$ & $\begin{array}{l}\mathrm{CD}^{+} 0^{+} / \mathrm{CD} 86^{+} / \\
\mathrm{CD}^{+} 0^{+} / \mathrm{MHCII}^{\text {high }}\end{array}$ & $\mathrm{CD} 206^{+}$ \\
\hline Dendritic cell & $\begin{array}{l}\mathrm{CD} 45^{+} / \mathrm{CD}_{11} \mathrm{~b}^{+-} / \\
\mathrm{CD}^{+} 1 \mathrm{c}^{+} / \mathrm{F} 4 / 80^{-} / \\
\text {autofluorescence }^{-}\end{array}$ & $\begin{array}{l}\mathrm{CD} 80^{+} / \mathrm{CD} 86^{+} / \\
\mathrm{CD}^{+} 0^{+} / \mathrm{MHCII}^{\mathrm{high}}\end{array}$ & \\
\hline B cell & $\begin{array}{l}\mathrm{CD} 45^{+} / \mathrm{CD} 19^{+} / \\
\mathrm{SSc}^{\text {low }}\end{array}$ & $\mathrm{CD} 9^{+}$ & \\
\hline $\mathrm{CD}^{+}{ }^{+} \mathrm{Th}$ cell & $\begin{array}{l}\mathrm{CD} 45^{+} / \beta \mathrm{TcR}^{+} / \\
\mathrm{CD}^{+} / \mathrm{SSc}^{\text {low }}\end{array}$ & $\mathrm{CD} 9^{+}$ & \\
\hline $\mathrm{CD}^{+} \mathrm{T}$ cell & $\begin{array}{l}\mathrm{CD} 45^{+} / \beta \mathrm{TcR}^{+} / \\
\mathrm{CD}^{+} / \mathrm{SSc}^{\text {low }}\end{array}$ & $\mathrm{CD} 9^{+}$ & \\
\hline$\gamma \delta \mathrm{T}$ cell & $\begin{array}{l}\mathrm{CD} 45^{+} / \gamma \delta \mathrm{TcR}^{+} / \\
\mathrm{SSc}^{\text {low }}\end{array}$ & $\mathrm{CD}^{+} 9^{+}$ & \\
\hline NK cell & $\begin{array}{l}\mathrm{CD} 45^{+} / \mathrm{NK} 1.1^{+} / \\
\mathrm{SSc}^{\text {low }}\end{array}$ & $\mathrm{CD} 9^{+}$ & \\
\hline
\end{tabular}




\section{Splenic Adherent Cell Enrichment}

Spleen cells were divided into two non-tissue culture petri plate and incubated at $37^{\circ} \mathrm{C}$ for two hours. Non-adherent cells were removed and cells incubated in cold PBS at $4^{\circ} \mathrm{C}$ for 15 minutes. Adherent cells were removed with a cell scraper, collected and pelleted at $1500 \mathrm{rpm}$ for 5 minutes then resuspended in media for cell stimulation assays.

\section{Spleen and Splenic Adherent Cell Stimulation}

Spleen cells $\left(5 \times 10^{5}\right.$ cells/well $)$ were plated in a 96 well plate and stimulated with SR $(0.025 \mathrm{mg} / \mathrm{mL})$ in the presence or absence of bafilomycin $(0.1 \mu \mathrm{M})$. In other experiments, spleen cells were stimulated with SR RNA complexed to DOTAP, a liposomal transfection agent at a concentration of $0.01,0.1,1,10 \mu \mathrm{g} / \mathrm{mL}$. Imiquimod $(0.25 \mu \mathrm{g} / \mathrm{mL})$ was used as a positive control. In all experiments, spleen cells were stimulated for 24 hours then cell culture supernatants were harvested and stored at $-80^{\circ} \mathrm{C}$ for analysis by ELISA. Enriched splenic adherent cells $\left(5 \times 10^{5}\right.$ cells/well $)$ were plated in a 96 well plate then stimulated with SR $(0.05 \mathrm{mg} / \mathrm{mL})$ for 24 hours and cell culture supernatants were harvested and stored at $-80^{\circ} \mathrm{C}$ for analysis by Luminex. Cytokine and chemokine concentrations were extrapolated from a standard curve and compared to cells cultured in media alone.

\section{Proliferation Assay}

SR and bafilomycin toxicity was measured using the CellTiter96 ${ }^{\circledR}$ Aqueous One Solution Cell Proliferation Assay (Promega, Madison, WI). Spleen cells $\left(2.5 \times 10^{5}\right.$ cells/well) were plated and stimulated with SR $(0.025 \mathrm{mg} / \mathrm{mL})$ in the presence or absence of bafilomycin $(1 \mu \mathrm{M})$. During the last 4 hours of culture, MTS-PMS solution was added to the well then absorbance read at $490 \mathrm{~nm}$ with a reference reading at $630 \mathrm{~nm}$. The absorbance was normalized to the average absorbance of WT spleen cells in media alone. A treatment was considered toxic if the absorbance was significantly reduced compared to cells without treatment.

\section{Bone Marrow Derived Macrophage (BMDM) Generation and Stimulation}

\section{Bone Marrow Harvest and Storage}

Bone marrow cells were harvested from the femur and tibia of WT and TLR2/9 ${ }^{-/}$ mice. Mice were sacrificed and fur completely wetted down with $70 \%$ ethanol. The fur, skin, and large muscle tissue was removed from the hind legs. Legs were removed at the hip and the paw removed at the joint to prevent cutting the femur and tibia. The bones were sprayed with ethanol and placed in media on ice until all mice were processed. In a sterile hood, any remaining flesh was removed from the bones. The epiphysis was cut and 
bone marrow flushed with sterile PBS into a $50 \mathrm{~mL}$ tube using a 26 gauge $1 / 2$ inch needle and syringe. The bone marrow was centrifuged at $400 \mathrm{~g}$ and supernatant decanted. The bone marrow cells were pipetted up and down to break up clumps and washed with sterile PBS and pelleted at 400g. The supernatant was discarded and RBCs lysed with RBC lysis buffer. Bone marrow cells were passed through a $70 \mu \mathrm{m}$ cell strainer (BD Biosciences, San Jose, CA) to obtain a single cell suspension. Cells were pelleted at $400 \mathrm{~g}$ and resuspended in media. Cells were counted by trypan dye exclusion on a hemocytometer and brought to a concentration of $6 \times 10^{6}$ cells $/ \mathrm{mL}$. Bone marrow cells were either plated immediately or reconstituted in freezing media ( $90 \%$ heat inactivated FCS, $10 \%$ DMSO) and frozen at $-80^{\circ} \mathrm{C}$ and transferred to liquid nitrogen for long term storage (Marim et al., 2010). To thaw bone marrow progenitor cells for BMDM differentiation, bone marrow progenitor cells were rapidly thawed in a $37^{\circ} \mathrm{C}$ water bath and were washed with media to removed DMSO.

\section{BMDM Differentiation}

To generate macrophages, bone marrow progenitor cells from one mouse (approximately $30 \times 10^{6}$ cells) were cultured in a non-tissue culture sterile petri plate in $15 \mathrm{~mL}$ BMDM differentiation media containing 30\% L-cell conditioned medium (LCCM) as a source of M-CSF (Table 2-5). The following day, the non-adherent cells were harvested, cells counted, and plated at $5 \times 10^{6}$ cells/plate in media containing $30 \%$ LCCM bone marrow differentiation media. On the fourth day, $5 \mathrm{~mL}$ of the media was removed and $10 \mathrm{~mL}$ of warm bone marrow differentiation media containing $60 \%$ LCCM was added so that the final concentration of the dish is 30\% LCCM. On day 6-7 the adherent cells were harvested and the purity of the macrophage $\left(\mathrm{CD} 11 \mathrm{~b}^{+} / \mathrm{F} 4 / 80^{+}\right)$preparation was determined by flow cytometry and was routinely between $80-85 \%$.

\section{BMDM Stimulation}

The BMDMs $\left(5 \times 10^{5}\right.$ cells/well $)$ were plated in BMDM cultivation media containing 5\% LCCM (Table 2-5). The BMDMs were allowed to adhere overnight then stimulated with SR $(0.05 \mathrm{mg} / \mathrm{mL})$ or imiquimod $(3 \mu \mathrm{g} / \mathrm{mL})$ for $24 \mathrm{hrs}$ in the presence or absence of bafilomycin $(0.1 \mu \mathrm{M})$, then cell culture supernatants were harvested and stored at $-80^{\circ} \mathrm{C}$ for analysis by ELISA. CpG DNA stimulation was used as a positive control.

\section{ELISA}

\section{Sandwich ELISA}

Cytokines present in unconcentrated BALF or culture supernatants were measured by ELISA according to manufacturer's instructions. ELISA plates (Nunc flat- 
Table 2-5. BMDM media.

\begin{tabular}{|c|c|c|c|c|}
\hline Reagent & $\begin{array}{c}\text { Differentiation } \\
\text { media }\end{array}$ & $\begin{array}{c}\text { Cultivation } \\
\text { media }\end{array}$ & Manufacturer & Location \\
\hline DMEM & $\mathrm{X}$ & $\mathrm{X}$ & $\begin{array}{l}\text { Cellgro, } \\
\text { Mediatech Inc. }\end{array}$ & $\begin{array}{l}\text { Manassas, } \\
\text { VA }\end{array}$ \\
\hline $\begin{array}{l}15 \% \text { heat } \\
\text { inactivated FCS }\end{array}$ & $\mathrm{X}$ & $\mathrm{X}$ & $\begin{array}{l}\text { Hyclone, Thermo } \\
\text { Scientific }\end{array}$ & Logan, UT \\
\hline $2 \mathrm{mM}$ glutamine & $\mathrm{X}$ & $\mathrm{X}$ & $\begin{array}{l}\text { Cellgro, } \\
\text { Mediatech Inc. }\end{array}$ & $\begin{array}{l}\text { Manassas, } \\
\text { VA }\end{array}$ \\
\hline $\begin{array}{l}\text { 100IU/mL } \\
\text { penicillin }\end{array}$ & $\mathrm{X}$ & $X$ & $\begin{array}{l}\text { Cellgro, } \\
\text { Mediatech Inc. }\end{array}$ & $\begin{array}{l}\text { Manassas, } \\
\text { VA }\end{array}$ \\
\hline $\begin{array}{l}100 \mu \mathrm{g} / \mathrm{mL} \\
\text { streptomycin }\end{array}$ & $X$ & $X$ & $\begin{array}{l}\text { Cellgro, } \\
\text { Mediatech Inc. }\end{array}$ & $\begin{array}{l}\text { Manassas, } \\
\text { VA }\end{array}$ \\
\hline $\begin{array}{l}1 \mu \mathrm{L} / \mathrm{L} \beta- \\
\text { mercaptoethanol }\end{array}$ & $\mathrm{X}$ & $\mathrm{X}$ & Fisher Scientific & $\begin{array}{l}\text { Waltham, } \\
\text { MA }\end{array}$ \\
\hline $\begin{array}{l}50 \mu \mathrm{g} / \mathrm{mL} \\
\text { gentamycin }\end{array}$ & $\mathrm{X}$ & & $\begin{array}{l}\text { GIBCO cell } \\
\text { culture, Invitrogen }\end{array}$ & Carlsbad, CA \\
\hline LCCM & $30 \%$ & $5 \%$ & & \\
\hline
\end{tabular}


bottom 96 well plate, Apogent, Portsmouth, NH) were coated with capture antibody then incubated with samples or standards diluted in a 7 point 2 -fold dilution series. Manufacturer and cytokine data range can be found in Table 2-6. After incubation, plates were washed to remove unbound proteins and cytokine specific biotinylated detection antibody added. Plates were again washed to remove unbound antibody, then cytokine bound biotinylated antibodies were incubated with avidin or streptavidin conjugated horseradish peroxidase. To visualize positive cytokine binding, 3,3',5,5'Tetramethylbenzidine (TMB) substrate (BD OptEIA, BD Biosciences, San Jose, CA) was added to the plate, which changes color in the presence of horseradish peroxidase. The color change reaction was stopped with $2 \mathrm{~N}$ sulfuric acid. The color change in each well of the plate was measured with a Spectra Max 340 plate reader (Molecular Devices, Sunnyvale, CA) at $450 \mathrm{~nm}$ and background absorbance at $570 \mathrm{~nm}$ was subtracted. For data analysis, a curve fit was applied to the standards and the sample concentrations extrapolated from the standard curve using four-parameter logistic software (SoftMax Pro, Sunnyvale, CA).

\section{Luminex/Bead Based ELISA}

For some experiments, cytokines were detected in BALF and cell culture supernatants using a Milliplex bead based ELISA assay (Millipore, Billerica, MA) according to the manufacturer's instructions. The cytokine standard mixture was reconstituted to $10,000 \mathrm{pg} / \mathrm{mL}$ and diluted in a 6 point 5 -fold dilution series $(10,000-$ $3.2 \mathrm{pg} / \mathrm{mL}$ ). Cytokine standards and samples were incubated with capture antibody conjugated fluorescent microbeads in a specially designed 96-well filter bottomed plate (Millipore). All incubations were done on a plate shaker to keep beads in solution. Vacuum suction was used to remove unbound proteins and wash buffer and beads incubated with cytokine specific biotinylated detection antibody. Plates were again washed to remove unbound antibody and cytokine bound biotinylated antibodies incubated with streptavidin-phycoerythrin (PE). Plates were washed and the mean fluorescence intensity of PE labeled cytokine bound beads was detected by flow cytometry with a Bio-Plex 200 system (Bio-Plex, R\&D, Minneapolis, MN). For data analysis, a curve fit was applied to the standards and the sample concentrations extrapolated from the standard curve using five-parameter logistic software (Bio-Plex Manager, R\&D, Minneapolis, MN).

\section{RNA Isolation}

\section{Lung Tissue}

Total RNA was extracted from the upper right lung lobe from individual mice using the Trizol method (Rio et al., 2010). Lung tissue was harvested, snap-frozen on dry ice, and stored at $-80^{\circ} \mathrm{C}$ until isolation. Trizol (Invitrogen, Carlsbad, CA) was added to 
Table 2-6. Enzyme linked immunosorbent assay (ELISA).

\begin{tabular}{lllll}
\hline Cytokine & Manufacturer & $\begin{array}{c}\text { Catalog } \\
\text { number }\end{array}$ & High standard & Low standard \\
\hline CXCL2/MIP-2 & R\&D Systems & DuoSet DY452 & $1000 \mathrm{pg} / \mathrm{mL}$ & $15.60 \mathrm{pg} / \mathrm{mL}$ \\
G-CSF & PeproTech & $900-\mathrm{K} 103$ & $2000 \mathrm{pg} / \mathrm{mL}$ & $31.25 \mathrm{pg} / \mathrm{mL}$ \\
IL-6 & BD Biosciences & OptEIA 555240 & $1000 \mathrm{pg} / \mathrm{mL}$ & $15.60 \mathrm{pg} / \mathrm{mL}$ \\
IL-10 & eBiosciences & $\begin{array}{l}\text { Ready-Set-Go } \\
88-7104\end{array}$ & $4000 \mathrm{pg} / \mathrm{mL}$ & $31.25 \mathrm{pg} / \mathrm{mL}$ \\
& & & & \\
IgM & Bethyl & E90-101 & $1000 \mathrm{ng} / \mathrm{mL}$ & $15.60 \mathrm{ng} / \mathrm{mL}$ \\
& $\begin{array}{l}\text { Laboratories } \\
\text { Inc }\end{array}$ & & & \\
BNF $\alpha$ & BD Biosciences & OptEIA 558534 & $1000 \mathrm{pg} / \mathrm{mL}$ & $15.60 \mathrm{pg} / \mathrm{mL}$ \\
\hline
\end{tabular}


the frozen tissue and immediately homogenized using an Omni TH tissue homogenizer (OMNI International, Kennesaw, GA). The tissue homogenizer was washed with 75\% ethanol and water between samples. The aqueous layer was separated using chloroform. RNA was precipitated with isopropanol, and RNA pellet washed with $75 \%$ ethanol twice. The RNA pellet was then dissolved in water and heated at $55^{\circ} \mathrm{C}$ for 10 minutes. Contaminating genomic DNA was removed by treatment with DNA-free (Ambion, Austin, TX) according to manufacturers' directions.

\section{Cultured Cells}

Culture media was removed completely and Trizol (Invitrogen) was added to the plate and allowed to incubate for 5 minutes. Trizol was then transferred to a microcentrifuge tube for RNA isolation. The aqueous layer was separated using chloroform. Glycogen was added to the aqueous RNA then both were precipitated with isopropanol. The RNA/glycogen pellet was washed with $75 \%$ ethanol twice. The $\mathrm{RNA} /$ glycogen pellet was then dissolved in water and heated at $55^{\circ} \mathrm{C}$ for 10 minutes. The amount of RNA recovered from cultured cells was small, and therefore the RNA was not DNAse treated.

\section{RNA Quantification and Quality}

\section{Spectrophotometer}

RNA concentration was determined using Ultrospec 2000 spectrophotometer (Pharmacia Biotech, Piscataway, NJ). RNA was diluted 1:25 in nuclease free water and the absorbance at $260 \mathrm{~nm}$ and $280 \mathrm{~nm}$ was measured. The concentration of RNA $(\mu \mathrm{g} / \mathrm{mL})$ is equal to the absorbance at $260 \mathrm{~nm}$ multiplied by the dilution factor (25) and $40 \mu \mathrm{g} / \mathrm{mL}$.

\section{Electrophoresis}

RNA quality was determined by running $2-3 \mu \mathrm{g}$ RNA in a $1 \%$ agarose gel. RNA was visualized with ethidium bromide under UV light (Alpha Image 2200 Multi-image light cabinet and alphaEase FC software, Alpha Innotech Corporation, San Leandro, CA). The presence of intact $18 \mathrm{~S}$ and $28 \mathrm{~S}$ ribosomal RNA bands was used as an indicator of high quality RNA.

\section{RT-PCR}

RNA $(2 \mu \mathrm{g})$ was reverse transcribed into cDNA with Promega Reverse Transcription System (Promega, Madison, WI). RNA was added to a master mix containing $\mathrm{MgCl}_{2}$, buffer, nucleotides, random primers, ribonuclease inhibitor, and avian 
myeloblastosis virus reverse transcriptase. To initiate reverse transcription, each RNA sample was incubated at room temperature for 15 minutes followed by $42^{\circ} \mathrm{C}$ for 30 minutes.

The cDNA $(1-3 \mu \mathrm{L})$ was added to a PCR master mix (Go Taq PCR core system I, Promega, Madison, WI) containing Taq polymerase, nucleotides, $15 \mu \mathrm{M}$ forward and reverse gene specific primers (Table 2-7), $\mathrm{MgCl}_{2}$, in a reaction buffer. The PCR reaction was carried out in a PCR thermocycler (Mastercycler epgradient S, Eppindorf, Hamburg, Germany). Each reaction began with a denaturation step of $94^{\circ} \mathrm{C}$ followed by an annealing step at primer specific temperature (Table 2-7) and an elongation step at $72^{\circ} \mathrm{C}$ repeated for 35 cycles.

The difference in gene expression was gauged by running $15 \mathrm{~mL}$ of the amplified RT-PCR product in a $1.8 \%$ agarose gel. RT-PCR product bands were visualized with ethidium bromide under UV light (Alpha Image 2200 Multi-image light cabinet and alphaEase FC software, Alpha Innotech Corporation, San Leandro, CA).

\section{Real Time qRT-PCR}

RNA (1 $1 \mu \mathrm{g})$ was reverse transcribed into cDNA with the Roche Transcriptor First Strand cDNA synthesis kit (Roche Applied Science, Indianapolis, IN) or Promega Reverse Transcription System (Promega, Madison, WI). RNA was added to a master mix containing buffer, nucleotides, random primers, ribonuclease inhibitor, and transcriptor reverse transcriptase. To initiate reverse transcription, each RNA sample was incubated at room temperature followed by $55^{\circ} \mathrm{C}$ or $42^{\circ} \mathrm{C}$ for 30 minutes then inactivated at $85^{\circ} \mathrm{C}$ for 5 minutes.

The cDNA $(2-3 \mu \mathrm{L})$ was added to an LC480 master mix (Roche Applied Science, Indianapolis, IN) or Kapa Probe Fast Universal (Kapa Biosystems, Woburn, MA) containing $5 \mu \mathrm{M}$ forward and reverse gene specific primers, and $10 \mu \mathrm{M}$ product specific probe. All primers and probes were chosen using the online software Universal Probe Library (Table 2-8). After each sample was loaded into a 96 well plate, the plate was spun down with a short spin and placed into a LightCycler 480 real time PCR thermal cycler (Roche Applied Science, Indianapolis, IN). Each real time reaction began with a denaturation step of $95^{\circ} \mathrm{C}$, followed by an annealing step at $60^{\circ} \mathrm{C}$, and an elongation step at $72^{\circ} \mathrm{C}$ repeated for 50 cycles. Data are normalized for HPRT and plotted as fold induction over unexposed control mice.

\section{Histology}

The left lobe of lung was removed and fixed with neutral buffered formalin and embedded in paraffin. Eight micron sections were cut and stained with hematoxylin and eosin (H\&E) to analyze granuloma formation or Masson's trichrome to visualize fibrosis 
Table 2-7. Primers used in RT-PCR.

\begin{tabular}{|c|c|c|c|c|}
\hline Primer name & Forward primer & Reverse primer & $\begin{array}{l}\text { Product } \\
\text { length }\end{array}$ & $\begin{array}{c}\text { Annealing } \\
\text { temp }\end{array}$ \\
\hline Actin & $\begin{array}{l}\text { GTG GGC CGC } \\
\text { TCT AGG CAC } \\
\text { CA }\end{array}$ & $\begin{array}{l}\text { CGG TTG GCC } \\
\text { TTA GGG TTC } \\
\text { AGG GGG G }\end{array}$ & $245 \mathrm{nt}$ & $60^{\circ} \mathrm{C}$ \\
\hline CCL11/eotaxin & $\begin{array}{l}\text { TTC TAT TCC } \\
\text { TGC TGC TCA } \\
\text { CGG }\end{array}$ & $\begin{array}{l}\text { AGG GTG CAT } \\
\text { CTG TTG TTG } \\
\text { GTG }\end{array}$ & $157 \mathrm{nt}$ & $57^{\circ} \mathrm{C}$ \\
\hline IL-4 & $\begin{array}{l}\text { TCG GCA TTT } \\
\text { TGA ACG AGG } \\
\text { TC }\end{array}$ & $\begin{array}{l}\text { GAA AAG CCC } \\
\text { GAA AGA GTC } \\
\text { TC }\end{array}$ & $261 \mathrm{nt}$ & $60^{\circ} \mathrm{C}$ \\
\hline TLR3 & $\begin{array}{l}\text { CTC ACC TCC } \\
\text { ACA TCT TGA } \\
\text { AT }\end{array}$ & $\begin{array}{l}\text { CTG TTC AGG } \\
\text { TTT TGA AAA } \\
\text { GG }\end{array}$ & $232 \mathrm{nt}$ & $55^{\circ} \mathrm{C}$ \\
\hline TLR4 & $\begin{array}{l}\text { ACC TCT GCC } \\
\text { TTC ACT ACA } \\
\text { GA }\end{array}$ & $\begin{array}{l}\text { AGG GAC TTC } \\
\text { TCA ACC TTC TC }\end{array}$ & $223 n t$ & $55^{\circ} \mathrm{C}$ \\
\hline TLR5 & $\begin{array}{l}\text { GAT CTT TCT } \\
\text { GAA AAT GGC } \\
\text { TG }\end{array}$ & $\begin{array}{l}\text { CTG GTC AGG } \\
\text { ATC TCT GAT GT }\end{array}$ & $144 n t$ & $55^{\circ} \mathrm{C}$ \\
\hline TLR7 & $\begin{array}{l}\text { GTT CAA AAA } \\
\text { CAA AGA GCC } \\
\text { AC }\end{array}$ & $\begin{array}{l}\text { AGG AAT GAA } \\
\text { AGA TGC TGA } \\
\text { AA }\end{array}$ & $132 \mathrm{nt}$ & $55^{\circ} \mathrm{C}$ \\
\hline
\end{tabular}


Table 2-8. Primers used in real time qRT-PCR.

\begin{tabular}{|c|c|c|c|c|}
\hline Primer name & Forward primer & Reverse primer & $\begin{array}{l}\text { Product } \\
\text { length }\end{array}$ & $\begin{array}{c}\text { Probe } \\
\text { number }\end{array}$ \\
\hline HPRT & $\begin{array}{l}\text { TCC TCC TCA GAC } \\
\text { CGC TTT T }\end{array}$ & $\begin{array}{l}\text { CCT GGT TCA TCA } \\
\text { TCG CTA ATC }\end{array}$ & $90 \mathrm{nt}$ & 95 \\
\hline CCL2/MCP-1 & $\begin{array}{l}\text { CAT CCA CGT GTT } \\
\text { GGC TCA }\end{array}$ & $\begin{array}{l}\text { GAT CAT CTT GCT } \\
\text { GGT GAA TGA GT }\end{array}$ & $76 \mathrm{nt}$ & 62 \\
\hline CCL4/MIP-1 $\beta$ & $\begin{array}{l}\text { GCC CTC TCT CTC } \\
\text { CTC TTG CT }\end{array}$ & $\begin{array}{l}\text { GAG GGT CAG AGC } \\
\text { CCA TTG }\end{array}$ & $73 \mathrm{nt}$ & 1 \\
\hline CXCL1/KC & $\begin{array}{l}\text { AGA CTC CAG CCA } \\
\text { CAC TCC AA }\end{array}$ & $\begin{array}{l}\text { TGA CAG CGC AGC } \\
\text { TCA TTG }\end{array}$ & $130 \mathrm{nt}$ & 83 \\
\hline CXCL2/MIP-2 & $\begin{array}{l}\text { CCT GGT TCA GAA } \\
\text { AAT CAT CCA }\end{array}$ & $\begin{array}{l}\text { CTT CCG TTG AGG } \\
\text { GAC AGC }\end{array}$ & $93 \mathrm{nt}$ & 63 \\
\hline $\mathrm{IFN} \gamma$ & $\begin{array}{l}\text { GGA GGA ACT GGC } \\
\text { AAA AGG AT }\end{array}$ & $\begin{array}{l}\text { TTC AAG ACT TCA } \\
\text { AAG AGT CTG AGG }\end{array}$ & $85 \mathrm{nt}$ & 21 \\
\hline IL-6 & $\begin{array}{l}\text { ATG GAT GCT ACC } \\
\text { AAA CTG GAT }\end{array}$ & $\begin{array}{l}\text { CCA GGT AGC TAT } \\
\text { GGT ACT CCA GA }\end{array}$ & $85 \mathrm{nt}$ & 6 \\
\hline IL-10 & $\begin{array}{l}\text { CAG AGC CAC ATG } \\
\text { CTC CTA GA }\end{array}$ & $\begin{array}{l}\text { GTC CAG CTG GTC } \\
\text { CTT TGT TT }\end{array}$ & $78 \mathrm{nt}$ & 41 \\
\hline IL-12p40 & $\begin{array}{l}\text { GAT TCA GAC TCC } \\
\text { AGG GGA CA }\end{array}$ & $\begin{array}{l}\text { TGG TTA GCT TCT } \\
\text { GAG GAC ACA TC }\end{array}$ & $75 \mathrm{nt}$ & 27 \\
\hline IL-17A & $\begin{array}{l}\text { TGT GAA GGT CAA } \\
\text { CCT CAA AGT CT }\end{array}$ & $\begin{array}{l}\text { GAG GGA TAT CTA } \\
\text { TCA GGG TCT TCA } \\
\text { T }\end{array}$ & $132 \mathrm{nt}$ & 50 \\
\hline IL-22 & $\begin{array}{l}\text { TTT CCT GAC CAA } \\
\text { ACT CAG CA }\end{array}$ & $\begin{array}{l}\text { CTG GAT GTT CTG } \\
\text { GTC GTC AC }\end{array}$ & $67 \mathrm{nt}$ & 17 \\
\hline IL-23p19 & $\begin{array}{l}\text { TCC CTA CTA GGA } \\
\text { CTC AGC CAA C }\end{array}$ & $\begin{array}{l}\text { TGG GCA TCT GTT } \\
\text { GGG TCT }\end{array}$ & $67 \mathrm{nt}$ & 19 \\
\hline $\mathrm{TNF} \alpha$ & $\begin{array}{l}\text { TCT TCT CAT TCC } \\
\text { TGC TTG TGG }\end{array}$ & $\begin{array}{l}\text { GGT CTG GGC CAT } \\
\text { AGA ACT GA }\end{array}$ & $128 \mathrm{nt}$ & 49 \\
\hline
\end{tabular}


in the lung. Slides were digitized to 40x resolution (Scanscope XT, Aperio, Vista, CA) and analyzed using Spectrum Imagescope software (Aperio, v11.2).

To quantify blue stained collagen on Masson's trichrome stained slides, $8 \times 1 \mathrm{~mm}^{2}$ square analysis areas were selected in Imagescope. The number of blue pixels was quantified using the positive pixel algorithm (v9.1) with a positive hue value of 0.65 , a hue width of 0.2 , and a color saturation threshold of 0.1 . All other settings were left at default values. Positive and strong positive pixels were quantified in each analysis area. A representative image from the analysis area with the median number of positive and strong positive pixels was captured at 40x.

\section{Statistics}

Data were analyzed using one-way ANOVA with Tukey-Kramer post hoc test or two-way ANOVA with Bonferroni post hoc test using GraphPad Prism statistical software (GraphPad Software, San Diego, CA). Differences were considered significant at $p$ values of less than 0.05 . Results are expressed as mean \pm S.D. 


\section{CHAPTER 3. ACUTE PHASE*}

\section{Introduction}

Acute hypersensitivity pneumonitis (HP) is characterized by neutrophilic alveolitis and inflammatory cytokine production. Neutrophils play an important role in the development of lung damage throughout the different stages of HP. They are the first cell population recruited into the lungs following SR exposure and they release numerous cytokines such as TNF $\alpha$ and IFN $\gamma$ that contribute to the initiation of disease (Fournier et al., 1985; Nance et al., 2005). Our previous studies using the SR model of HP demonstrated that MyD88-dependent receptors are necessary for the initial production of pro-inflammatory cytokines, neutrophil chemokines and subsequently neutrophil recruitment following acute SR exposure (Nance et al., 2008). One of the MyD88dependent TLRs involved in recognition of gram positive bacteria is TLR2, and our previous studies revealed that SR interacts with TLR2 and can activate the TLR2 signaling pathway that leads to NF- $\mathrm{KB}$ activation using a luciferase reporter assay in vitro. Mice deficient in TLR2 have a significant reduction in the neutrophil chemokine CXCL2/MIP-2 but not neutrophil recruitment. TLR9 has previously been shown to play an important role in the immune response to mycobacteria and fungus induced HP (Bhan et al., 2011; Daito et al., 2011). Therefore, we hypothesize TLRs 2 and 9 cooperate in SR induced chemokine production and neutrophil recruitment.

\section{Toll-Like Receptors}

Toll-like receptors (TLRs) are pattern recognition receptors that recognize conserved molecular patterns present in bacteria, fungi, protozoa, and viruses. TLR1, TLR2, TLR4, TLR5, and TLR6 are present on the cell surface, whereas TLR3, TLR7, TLR8, TLR9, TLR11, TLR12, and TLR13 are present in endosomes. TLR2 forms heterodimers with TLR1 or TLR6 to recognize peptidoglycan and lipoproteins commonly found in gram positive bacteria. TLR4 recognizes LPS found in gram negative bacteria. TLR5 recognizes flagellin. In the endosome, TLR3 recognizes double stranded RNA found in viruses. TLR7 and TLR8 recognize viral and bacterial single stranded RNA. TLR9 recognizes the $\mathrm{CpG}$ motif found in bacterial and viral DNA. TLR11 and TLR12, present in mice but not humans, have been shown to recognize profilin, a protein found in the parasite T. gondii (Koblansky et al., 2013; Plattner et al., 2008; Yarovinsky et al., 2005). In addition TLR11 has also been shown to recognize bacterial flagellin (Mathur et

*Adapted with permission from Andrews K, Abdelsamed H, Yi A-K, Miller MA, Fitzpatrick EA (2013) TLR2 Regulates Neutrophil Recruitment and Cytokine Production with Minor Contributions from TLR9 during Hypersensitivity Pneumonitis. PLoS ONE 8(8): e73143. 
al., 2012). TLR13, also present in mice, has recently been shown to recognize bacterial 23S rRNA (Hidmark et al., 2012; Li and Chen, 2012). TLRs can be grouped according to whether the receptor signals through the adaptor molecule MyD88 or is MyD88independent. MyD88-dependent receptors include TLR2/1, TLR2/6, TLR5, TLR7, TLR8, TLR9, TLR11, TLR12, TLR13, whereas TLR3 is MyD88-independent. TLR4 can signal in a MyD88-dependent and MyD88-independent manner. Signaling through the MyD88 adaptor protein results in activation of mitogen-activated protein kinase (MAPK), NF-kB, and interferon regulatory factors (IRF) for the induction of inflammatory cytokines and type I interferons (reviewed in Kawai and Akira, 2010).

In the lung, TLR2 and TLR9 are expressed by dendritic cells as well as bronchial and airway epithelial cells and can be upregulated in response to infection (reviewed in Rohmann et al., 2011). Alveolar macrophages express TLR2 but have low levels of TLR9 (Suzuki et al., 2005). TLR7 is expressed in plasmacytoid dendritic cells (pDCs); however, recent reports have demonstrated that TLR7 can be expressed by other cells during chronic inflammation (Cherfils-Vicini et al., 2010; Hammadi et al., 2008; Nicholas et al., 2011; Nicholas et al., 2010). It is not clear what the role of TLR7 expression in these non-native cells is in regards to inflammation and host defense. TLR7 is widely recognized to be important in viral pathogen recognition; however, there have been reports that TLR7 may also recognize bacterial RNA and play a role in host defense against bacteria (Inoue et al., 2011; Mancuso et al., 2009; Rad et al., 2009). Sakai et al demonstrated that exposure to non-typeable Haemophilus influenzae upregulates TLR7 expression in the human airway epithelial cells and mouse whole lung in a TLR2 dependent manner (Sakai et al., 2007). Although it is becoming clear that TLR7 may play a role in the immune response to bacteria there is very little information on that role and our studies will begin to address this important question.

\section{Leukocyte Recruitment}

\section{Neutrophils}

Neutrophil recruitment from the circulation is a multistep process (reviewed in Kolaczkowska and Kubes, 2013). First, upregulation on selectins on endothelial cells initiates leukocyte rolling. P-selectin and E-selectin are upregulated on endothelial cells in response to inflammatory stimuli, cytokines, or PRRs. P-selectin and E-selectin bind to glycoproteins on leukocytes such as PSGL-1. Second, integrins bind to cell adhesion molecules. Integrins consist of two chains, an alpha chain and a beta chain. On neutrophils, the beta integrin $\beta 2 / \mathrm{CD} 18$ can pair with the alpha integrin $\alpha \mathrm{L} / \mathrm{CD} 11 \mathrm{a}$ and $\alpha \mathrm{M} / \mathrm{CD} 11 \mathrm{~b}$. CD11a/CD18 and CD11b/CD18 plays an important role in neutrophil recruitment by binding to the intracellular adhesion molecule (ICAM)-1, ICAM-2, and ICAM-3 expressed on endothelial cells to induce firm binding. Finally, neutrophils spread and cross the endothelial barrier in a process known as diapedesis. 
Neutrophils reach the site of infection by following a chemokine gradient. The chemokines form a gradient and attract leukocytes to sites of inflammation through the binding of chemokine receptors. Neutrophils express many chemokine receptors including CXCR2, which binds to the chemokines CXCL1/KC, CXCL2/MIP-2, and CXCL5/ENA-78. Previous studies revealed the importance of TLR2 in neutrophil recruitment in HP as mice deficient in TLR2 had significantly reduced CXCL2/MIP-2 production accompanied by a partial reduction in neutrophil recruitment following one SR exposure (Nance et al., 2008). However, since TLR2 deficiency only affected CXCL2 production whereas MyD88 $8^{-/-}$mice had a complete absence of both proinflammatory cytokine production and neutrophil recruitment following SR exposure, we will investigate the cooperativity of TLRs 2 and 9 in neutrophil recruitment and the production of neutrophil chemokines in HP. We hypothesize TLR2 $/ 9^{-1-}$ mice will have a significant reduction in neutrophil recruitment and neutrophil chemokine production after one exposure to SR.

\section{Monocytes}

Monocytes are also recruited to sites of inflammation in a manner similar to neutrophils; however monocytes express different chemokine receptors, and therefore are attracted by alternative chemokine gradients (reviewed in Shi and Pamer, 2011). Monocytes express the chemokine receptors CCR1, CCR2, and CCR5, which overlap in ligand specificity. CCR1 binds to CCL3/MIP-1 $\alpha$, CCL5/RANTES, and CCL7/MCP-3. CCR2 binds CCL2/MCP-1, CCL8/MCP-2, and CCL7/MCP-3. CCR5 binds CCL3/MIP-1 $\alpha$, CCL4/MIP-1 $\beta$, CCL5/RANTES, and CCL11/eotaxin (reviewed in Charo and Ransohoff, 2006). After a single dose of SR, mice have shown increases in the expression of CCL3/MIP-1 $\alpha$, and CCL2/MCP-1 (Nance et al., 2008; Schuyler et al., 2000), and SR stimulated alveolar macrophages produced CCL3/MIP-1 $\alpha$ in a dose dependent manner (Schuyler et al., 2000). In the present study, we will investigate the contribution of TLRs 2 and 9 to the production of monocyte chemokines CCL2/MCP-1, CCL3/MIP-1 $\alpha$, and CCL4/MIP-1 $\beta$ in response to SR exposure.

\section{Inflammatory Cytokines}

Inflammatory cytokines TNF $\alpha$, IL-6, IL-1 $\alpha$, and IL-12p40 (the common chain for IL-12 and IL-23) are detected in the BALF within 2 hours of SR exposure and remain elevated up to 72 hours later (Schuyler et al., 2000). Alveolar macrophages are one of the first cell types to encounter SR and are a source of inflammatory cytokines. Macrophages isolated from mice exposed to SR spontaneously release IL-1, IL-6, and TNF $\alpha$, and in vitro stimulation with SR induces greater IL-1, IL-6, and TNF $\alpha$ production (Denis et al., 1992). Previous studies in MyD88 ${ }^{-/}$mice revealed IL- $1 \beta$ and TNF $\alpha$ expression was dependent on MyD88; however $\mathrm{TLR}^{-1-}$ mice did not have a significant reduction in TNF $\alpha$ in the BALF at 6hrs and 24hrs compared to WT (Nance et al., 2008). Therefore, we hypothesize that in addition to TLR2, TLR9 also contributes to inflammatory cytokine production in SR exposed mice. 
In the following studies, the objective is to analyze neutrophil recruitment, inflammatory cytokine, and chemokine production after a single exposure to SR. We hypothesize the combined loss of TLRs 2 and 9 will result in reduced neutrophil recruitment due to reduced neutrophil and monocyte chemokine production, and reduced inflammatory cytokine production in vitro and in vivo.

\section{Results}

\section{TLRs 2 and 9 Contribute to Neutrophil Chemokine Production and Recruitment into the Airways Following One Exposure to SR In Vivo}

Our previous studies demonstrated that neutrophil recruitment was dependent on MyD88 signaling pathways, and TLR2 was one of the MyD88-dependent PRRs that contributed to CXCL2 production following a single SR exposure (Nance et al., 2008). To examine the combined role of TLRs 2 and 9 in neutrophil recruitment following in vivo exposure to SR, we exposed WT, TLR2 ${ }^{-/}$, $\mathrm{TLR}^{-/-}$, and TLR2 $/ 9^{-/-}$mice to SR and measured the recruitment of neutrophils into the airways (Figure 3-1). Analysis of the BAL from individual mice demonstrated that following a single exposure to SR, there is an increase in alveolitis in the WT mice, which is significantly reduced in the TLR $2 / 9^{-/-}$ mice. Although the $\mathrm{TLR}^{-1-}$ mice and $\mathrm{TLR}^{-/-}$mice had reduced alveolitis compared to the WT mice, the differences were not statistically significant (Figure 3-1A). At this time point the alveolitis is almost entirely neutrophilic. The reduction in alveolitis in the TLR $2 / 9^{-/-}$mice correlated with the decrease in neutrophil recruitment that was observed compared to WT mice (Figure 3-1B). Natural killer (NK) cells and $\gamma \delta \mathrm{T}$ cells are lymphocytes that can contribute to the innate immune response. A small number of $\mathrm{NK}$ cells were detected in the BAL of SR exposed mice, but there was no significant difference between WT and KO groups (Figure 3-1B). $\gamma \delta \mathrm{T}$ cells were not detected at this time point. Therefore, both TLRs 2 and 9 appear to contribute to neutrophil recruitment following a single SR exposure since a significant reduction in neutrophils was only observed in the TLR $2 / 9^{-/-}$mice.

To analyze the expression of neutrophil chemokines, lung tissue from WT, $\mathrm{TLR}^{-/-}$, TLR9 ${ }^{-/-}$, and TLR2 $/ 9^{-/-}$mice was harvested and the expression of neutrophil chemokines CXCL1 and CXCL2 was measured. Quantitative RT-PCR analysis revealed that there is a significant decrease in CXCL2 mRNA in the TLR2 ${ }^{-/-}$and TLR2 $/ 9^{-/-}$mice; however, there was no significant decrease in CXCL1 mRNA in any of the mutant mice (Figure 3-1C). The decrease in CXCL2 mRNA was reflected in the BALF; TLR2 ${ }^{-1}$, TLR $^{-/}$and TLR2 $/ 9^{-1-}$ mice had a significant decrease in CXCL2 in the BALF following SR exposure (Figure 3-2). Although there was no difference in expression of CXCL1, the concentration of CXCL1 in the BALF was also significantly reduced in $\mathrm{TLR}^{-/-}$, TLR $9^{-/-}$, and TLR $2 / 9^{-/-}$mice. The decrease in neutrophil recruitment was likely due to a decrease in chemokine production and not an intrinsic defect in TLR $2 / 9^{-l-}$

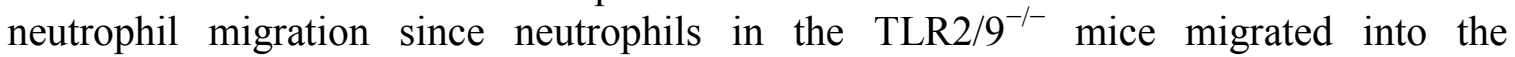


A
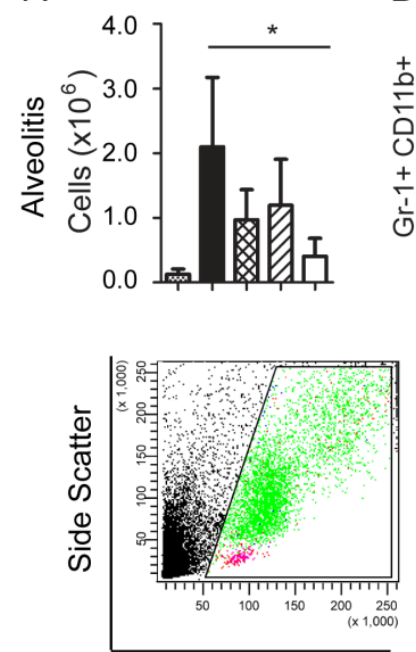

Forward Scatter

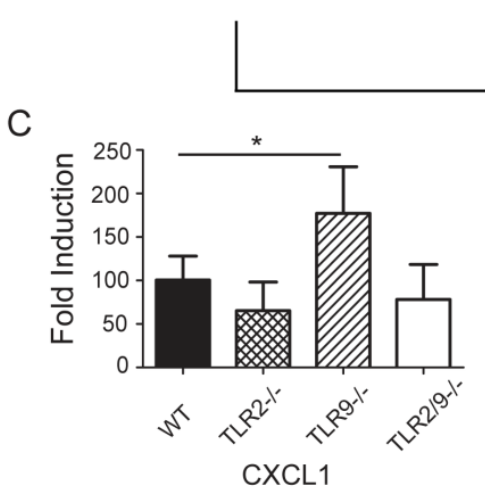

B
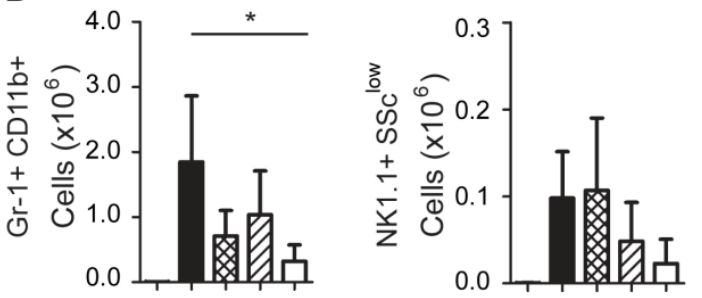

WT Unexp

WT SR Exp

区ख TLR2 ${ }^{-/-}$SR Exp

[7 $\mathrm{TLR}^{-/-} \mathrm{SR}$ Exp

口TLR2/9/- SR Exp

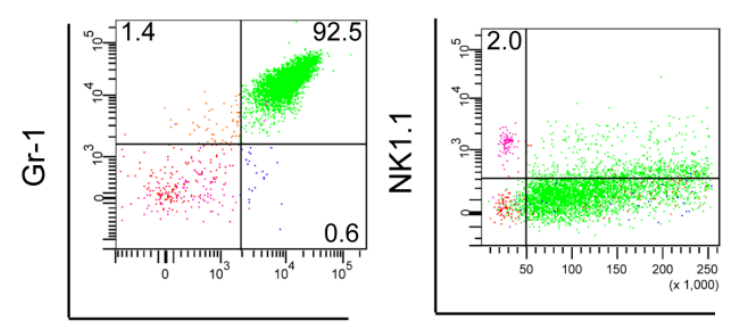

Side Scatter

CD11b

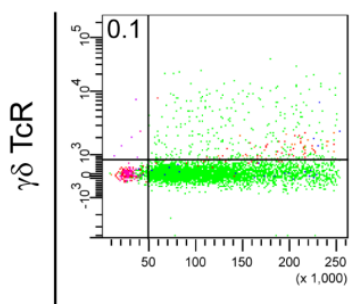

Side Scatter
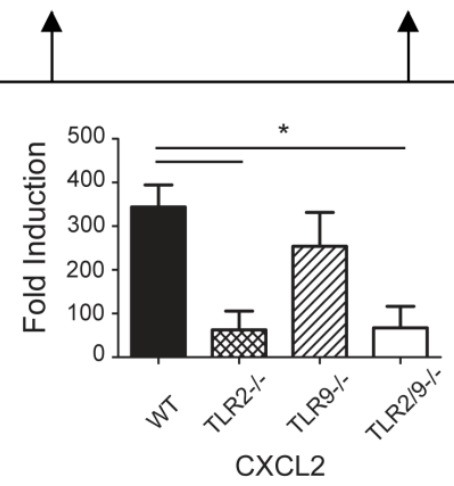

Figure 3-1. Neutrophil recruitment and chemokine expression after one exposure to $\mathrm{SR}$.

$\mathrm{WT}, \mathrm{TLR} 2^{--}$, $\mathrm{TLR}^{-/-}$, and TLR2 $/ 9^{-/-}$mice were exposed to SR and analyzed 6 hours after one exposure. (A) BAL was performed and alveolitis determined using trypan blue dye exclusion. (B) Cells recovered from the BAL fluid were incubated with antibody to CD11b, Gr-1, $\gamma \delta \mathrm{TcR}$, and NK1.1 and analyzed by flow cytometry to determine the number of neutrophils $\left(\mathrm{CD} 11 \mathrm{~b}^{+} / \mathrm{Gr}-1^{\text {high }}\right)$, and natural killer cells $\left(\mathrm{NK} 1.1^{+} / \mathrm{SSc}^{\text {low }}\right)$ in each group using the depicted gating strategy (Abdelsamed et al., 2011). $\gamma \delta$ T cells were not detected. (C) Expression of mRNA for cytokines was determined by qRT-PCR on RNA isolated from individual lung lobes ( $\mathrm{n}=3-5$ mice per group) and is expressed as fold induction over WT unexposed mice. The data represent mean \pm SD of duplicate samples and significance was determined using one-way ANOVA with Tukey post-hoc test $(* p<0.05$ compared to WT / SR exposed mice). 

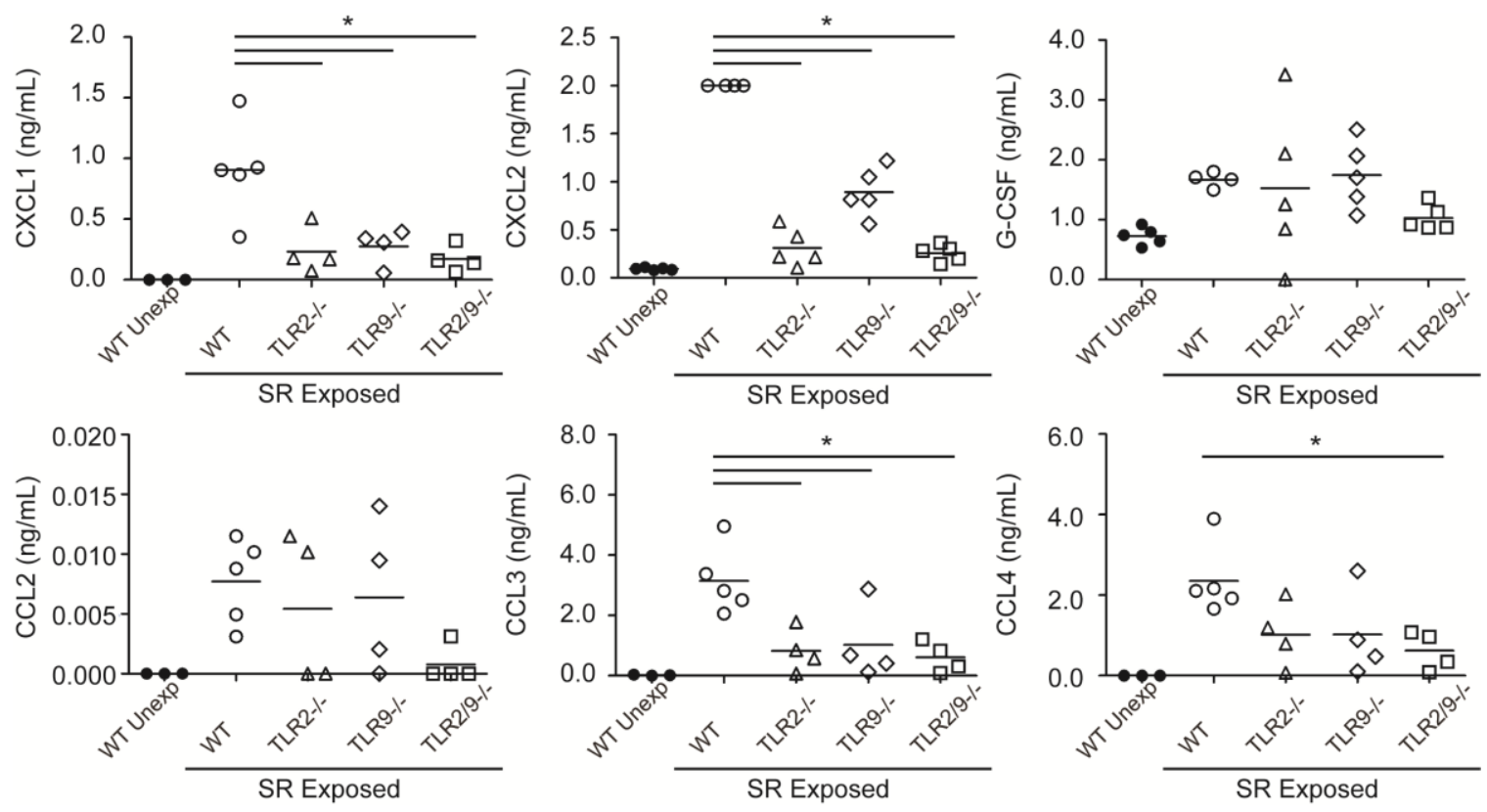

Figure 3-2. Chemokine production after one exposure to SR.

WT, $\mathrm{TLR}^{-/-}$, $\mathrm{TLR}^{-/-}$, and TLR2 $/ 9^{-/-}$mice were exposed to SR and analyzed 6 hours after one exposure. The cell-free BAL fluid was analyzed for CXCL2, G-CSF by ELISA and CXCL1, CCL3 and CCL4 by Milliplex bead based ELISA (limit of detection $0.0032 \mathrm{ng} / \mathrm{mL})$. Data represent the mean $\pm \mathrm{SD}(\mathrm{n}=3-5$ per group $)$ significance was determined using one-way ANOVA with Tukey post-hoc test $\left({ }^{*} p<0.05\right.$ compared to WT SR exposed mice). 
peritoneum in response to an IP injection of casein (Figure 3-3). We also observed a decrease in the chemokines CCL3 and CCL4 in the TLR2/ $/ 9^{-/-}$mice compared to the WT mice suggesting that TLRs 2 and 9 may also be important for recruitment of monocytes and lymphocytes following a single SR exposure. We did not find a significant decrease in G-CSF in the BALF of exposed mice (Figure 3-2). This suggests that lack of the neutrophil growth factor was not the cause of decreased neutrophil recruitment in the mice.

To confirm the reduction in neutrophil recruitment in TLR2/9 $9^{-/-}$mice was not due to increased neutrophil apoptosis, we exposed mice to SR once per day for 3 consecutive days and analyzed the percentage of neutrophils expressing the apoptosis marker annexin $\mathrm{V}$ by flow cytometry. Neutrophils were identified as CD $11 \mathrm{~b}^{+} / \mathrm{Gr}-1^{\text {high }}$ expressing cells. The results show SR exposed TLR2 $/ 9^{-/-}$mice had a decreased percentage of neutrophils in the BAL cells compared to WT exposed mice (Figure 3-4A); however, we did not see an increase in Annexin V staining on neutrophils from TLR2/9 ${ }^{-/-}$exposed mice compared to WT exposed mice (Figure 3-4B). The results suggest that increased apoptosis is not playing a role in the decrease in neutrophil frequency in the BALF of these mice. Altogether, these results suggest that following a single SR exposure, neutrophil chemokine production and recruitment is largely dependent on TLR2 and any role for TLR9 becomes apparent only in the absence of TLR2.

\section{TLR2 $/ 9^{-/-}$Mice Have a Significant Reduction in Inflammatory Cytokine Expression In Vivo}

The expression of cytokines was examined in the lung tissue of mice exposed once to SR. The expression of inflammatory cytokines TNF $\alpha$, IL- 6 and the antiinflammatory cytokine IL-10 was increased in mice exposed to SR over WT unexposed mice. There was no significant difference in the expression of TNF $\alpha$ and IL-6 in TLR2 ${ }^{-/}$, TLR9 ${ }^{-/}$, or TLR $2 / 9^{-1-}$ mice compared to WT exposed mice. IL-10 expression was significantly decreased in TLR2 ${ }^{-/}$and TLR $2 / 9^{-/-}$mice compared to WT exposed mice (Figure 3-5). The concentration of these cytokines in the BALF of mice exposed once to SR was measured. Although there was no significant difference in TNF $\alpha$ and IL-6 expression in the lung tissue, the BALF from $\mathrm{TLR}^{-/-}$and TLR2/9 ${ }^{-/-}$mice had a significant reduction in TNF $\alpha$ and TLR2/9 ${ }^{-/-}$mice had a significant reduction in IL-6 compared to WT exposed mice (Figure 3-6). IL-10 was not detected in the BALF at this time point. Altogether, the results suggest TLR2 is the dominant receptor responsible for the induction of TNF $\alpha$ and IL- 6 production, but TLR9 also contributes to the production of IL-6 after a single exposure to SR.

IL-12p40 is the common chain for heterodimeric cytokines IL-12p70 (IL-12p35 and IL-12p40) and IL-23 (IL-23p19 and IL-12p40). IL-12p70 and IL-23 are important for the polarization and proliferation of Th1 and Th17 cells respectively. IL-12p40 and IL-23p19 expression was significantly decreased in TLR2 ${ }^{-/-}$and TLR2 $/ 9^{-/-}$mice compared to WT exposed mice (Figure 3-5), suggesting TLR2 is important for IL-12p40 


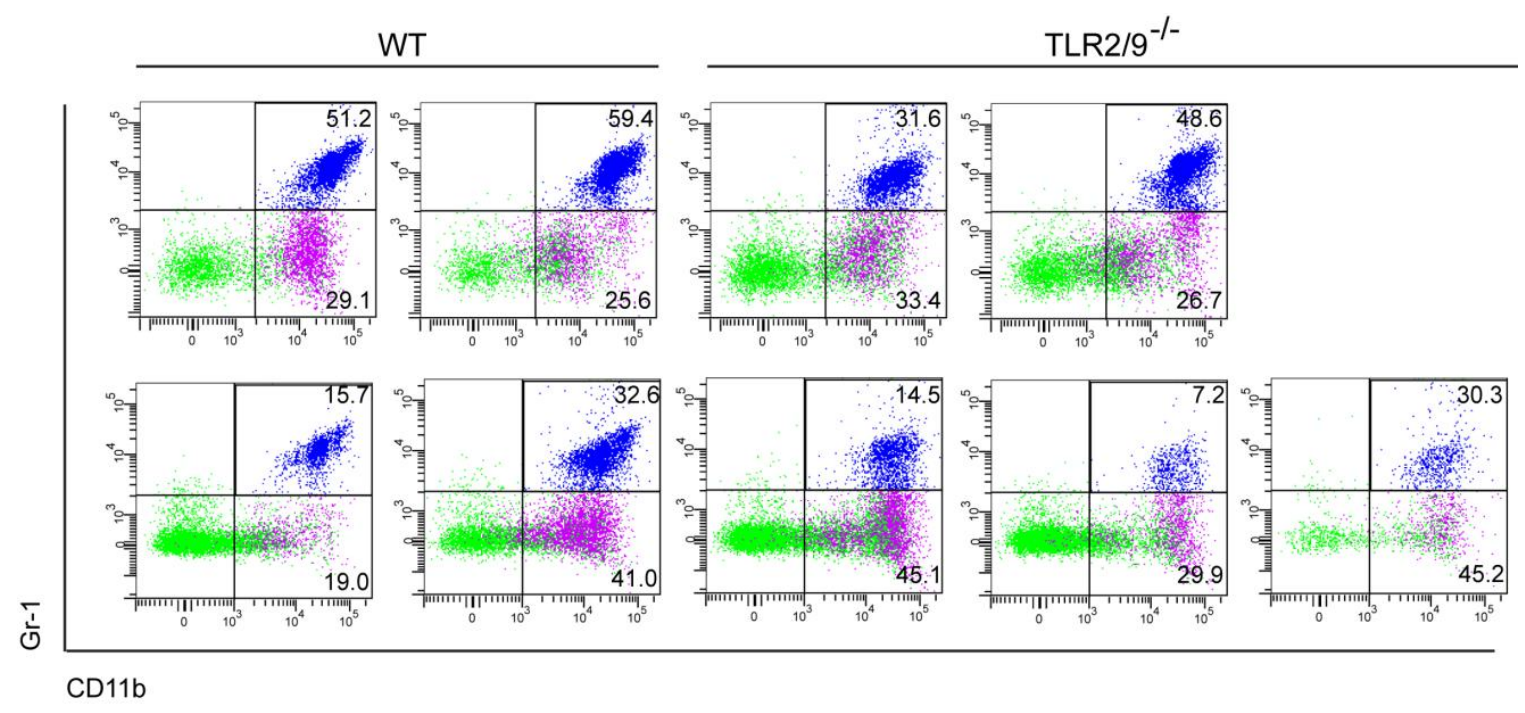

Figure 3-3. Neutrophils are recruited to the peritoneum in response to casein injections in WT and TLR2/9 ${ }^{-/-}$mice.

WT and TLR2 $/ 9^{-/-}$mice were injected IP with $1 \mathrm{~mL}$ of a $9 \%$ casein solution, followed by a second injection 24 hours later then sacrificed 3 hours after the last injection. Peritoneal lavage was performed, and cells recovered from the peritoneal lavage were incubated with antibody to $\mathrm{CD} 11 \mathrm{~b}$ and Gr-1 and analyzed by flow cytometry to determine the percentage of neutrophils (CD11 $\left.\mathrm{b}^{+} / \mathrm{Gr}-1^{\text {high }}\right)$ in each group. Each scatter plot represents a separate mouse $(n=4-5$ per group). 

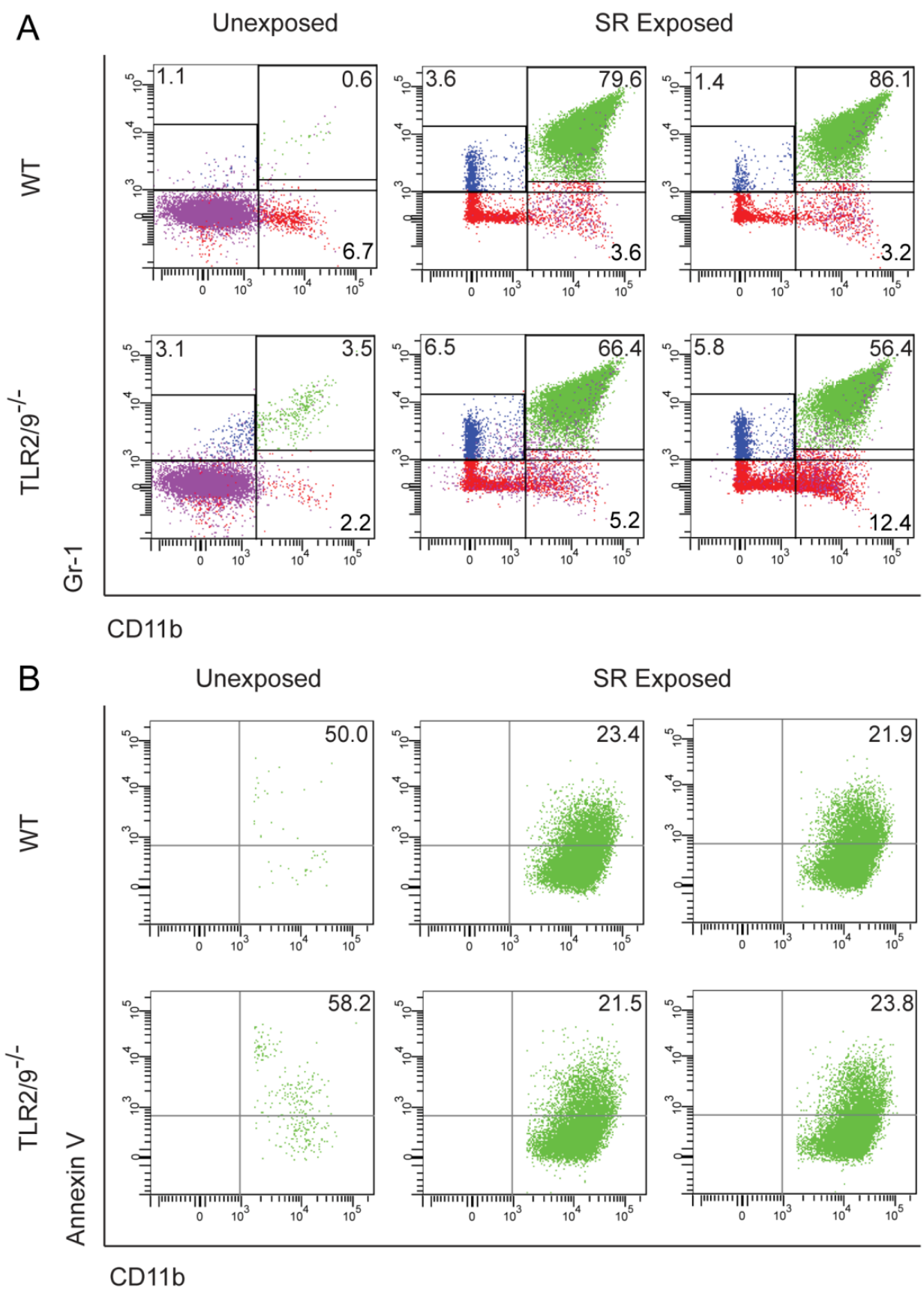

Figure 3-4. The decrease in neutrophil recruitment in $\mathrm{TLR}^{-1 / 9^{-1}}$ mice is not due to increased neutrophil apoptosis.

WT and TLR2 $/ 9^{-/-}$mice were exposed to SR three times and sacrificed 12 hours after the last exposure. BAL was performed and the cells recovered from the BAL fluid were incubated with antibody to CD11b, Gr-1, and annexin V and analyzed by flow cytometry. (A) Cells were gated on neutrophils $\left(\mathrm{CD} 11 \mathrm{~b}^{+} / \mathrm{Gr}-1^{\text {high }}\right)$ (B) The percentage of neutrophils $\left(\mathrm{CD} 11 \mathrm{~b}^{+} / \mathrm{Gr}-1^{\text {high }}\right)$ positive for annexin $\mathrm{V}$ staining in $\mathrm{WT}$ and TLR2 $/ 9^{---}$mice. $(\mathrm{n}=1-2$ per group) 

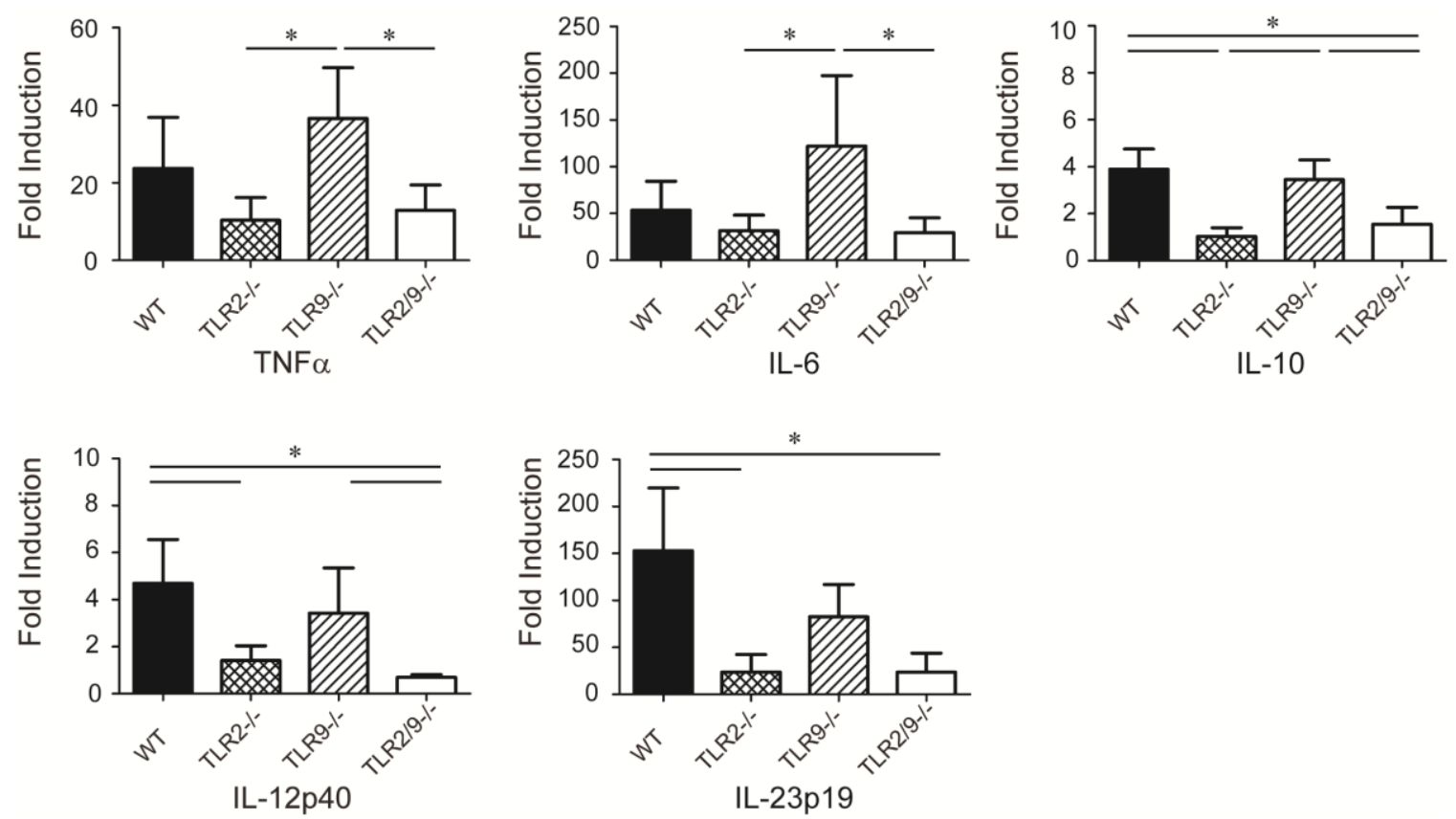

Figure 3-5. Inflammatory cytokine expression after one exposure to SR.

WT, TLR2 ${ }^{-/-}$, $\mathrm{TLR}^{-/-}$, and TLR2 $/ 9^{-/-}$were exposed to SR once and sacrificed 6 hours later. RNA was isolated from lung tissue and reverse transcribed to cDNA for real time PCR. The expression of TNF $\alpha$, IL-6, IL-10, IL-12p40, and IL-23p19 was normalized to HPRT. Data is expressed as fold induction over WT unexposed mice \pm SD. ( $n=5$ mice per group) and significance was determined using one-way ANOVA with Tukey posthoc test $(* p<0.05)$. 

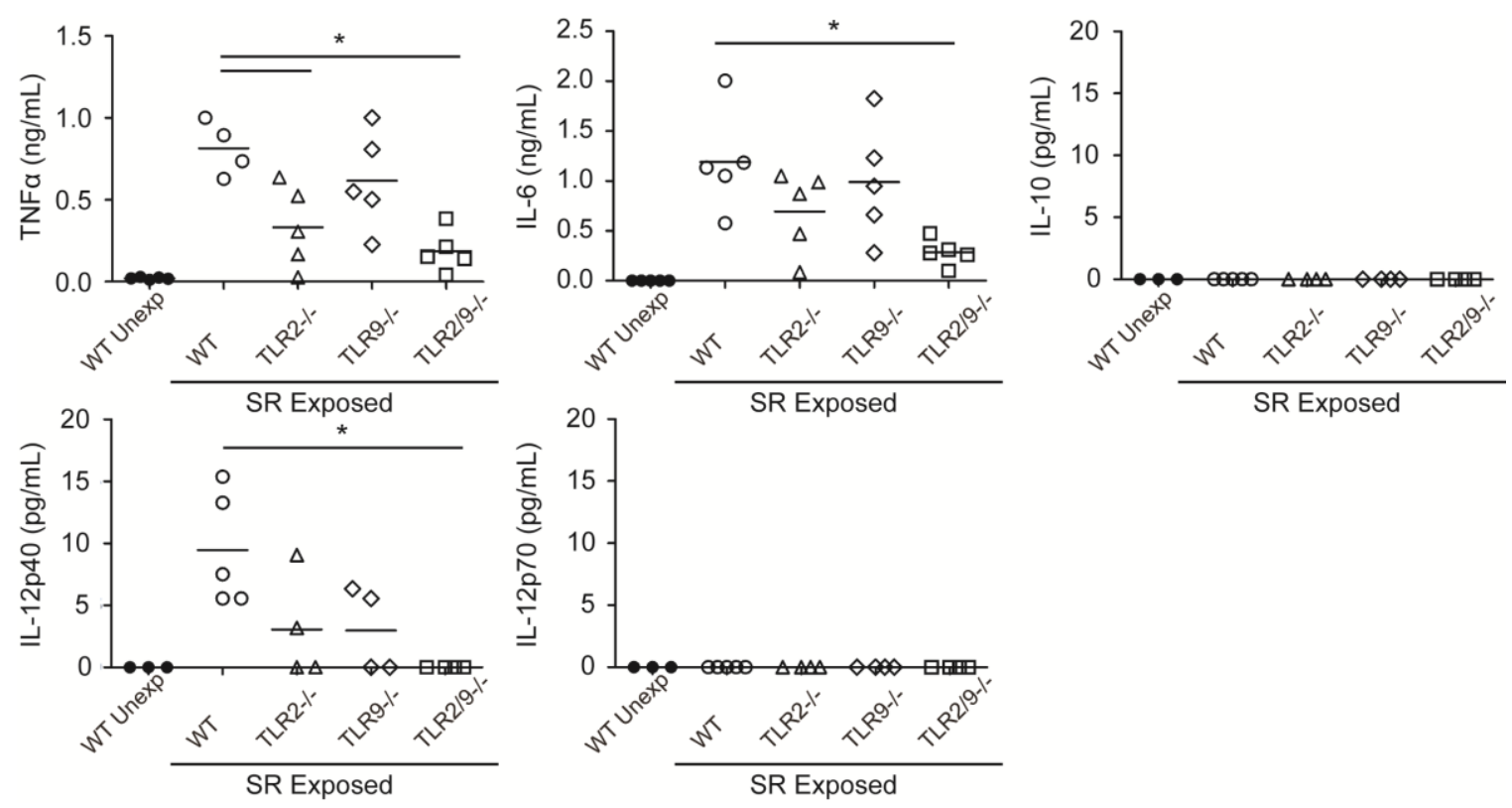

Figure 3-6. Inflammatory cytokine production after one exposure to SR.

$\mathrm{WT}, \mathrm{TLR} 2^{-/-}$, $\mathrm{TLR}^{-/-}$, and TLR $2 / 9^{-/-}$mice were exposed to SR and analyzed $6 \mathrm{~h}$ after one exposure. The cell-free BAL fluid was analyzed for TNF $\alpha$ and IL- 6 by ELISA and IL-10, IL-12p40, and IL-12p70 by Milliplex bead based ELISA (limit of detection $3.2 \mathrm{pg} / \mathrm{mL})$. Data represent the mean $\pm \mathrm{SD}(\mathrm{n}=3-5$ per group) significance was determined using one-way ANOVA with Tukey post-hoc test $\left({ }^{*} p<0.05\right.$ compared to WT SR exposed mice). 
and IL-23 expression. The concentration of IL-12p40 was significantly reduced in the BALF of TLR2 $/ 9^{-/-}$mice (Figure 3-6); however, IL-12p70 was not detected, suggesting the significant reduction was in the production of IL-23. The results suggest TLR2 contributes to the expression of IL-12p40, and IL-23p19 after a single exposure to SR and that loss of TLR2 may result in reduced Th17 responses during the granulomatous phase.

\section{TLR2/9-Dependent and MyD88-Dependent Chemokine and Cytokine Production in SR Stimulated Splenic Adherent Cells In Vitro}

Although neutrophil recruitment and inflammatory cytokine production was significantly reduced in TLR $2 / 9^{-/-}$mice compared to WT exposed mice, the reduction was not to the level previously shown in $\mathrm{MyD}^{-/-}$mice (Nance et al., 2008). To investigate MyD88-dependent cytokine production in response to SR, splenic adherent

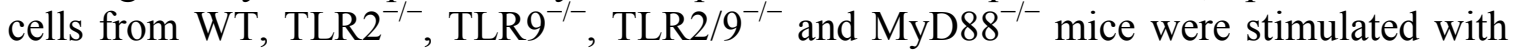
$\mathrm{SR}$ and cytokine production measured by bead based ELISA.

Neutrophil chemokines CXCL1, CXCL2, and the neutrophil growth factor G-CSF were partially dependent on TLRs 2 and 9 as the production of these proteins were significantly reduced in TLR2 ${ }^{-/-}$, TLR9 ${ }^{-/}, \mathrm{TLR} 2 / 9^{-/-}$cells, but completely dependent on MyD88 as protein expression was reduced to WT unexposed levels (Figure 3-7). The results indicate an additional MyD88-dependent receptor is involved in the induction of neutrophil chemokines and growth factors. GM-CSF production was not significantly induced by SR stimulation of splenic adherent cells compared to unexposed cells. The lymphocytic chemokines CXCL9 and CXCL10 were significantly increased in TLR9 ${ }^{-1}$, TLR2 $/ 9^{-/-}$, and MyD88 ${ }^{-/-}$cells compared to WT cells, suggesting signaling through TLR9 is important in inhibiting expression of CXCL9 and CXCL10 in splenic adherent cells (Figure 3-7).

The induction of MyD88-dependent cytokines was also examined in splenic adherent cells. CXCL9 and CXCL10 are induced in response to IFN $\gamma$ (Farber, 1997), and $\mathrm{TLR}^{-/-}$and TLR2 $/ 9^{-/-}$cells had a significant increase in the production of IFN $\gamma$ over WT SR stimulated cells. However, IFN $\gamma$ was not significantly increased MyD $88^{-/-}$cells (Figure 3-8), suggesting TLR9 stimulation suppresses the production of IFN $\gamma$, but an additional MyD88-dependent receptor is responsible for IFN $\gamma$ induction. The production of IL-1 $\beta$ and IL-17 after SR stimulation was significantly increased in TLR9 ${ }^{-/}$cells compared to WT cells (Figure 3-8). TLR2 ${ }^{-1-}, \mathrm{TLR}^{-/-}$, and TLR $2 / 9^{-/-}$cells had significantly increased production of IL-1 $\beta$ and IL-17 over MyD88 $8^{-/-}$cells suggesting a MyD88-dependent receptor is responsible for the induction of IL-1 $\beta$ and IL-17 production. SR stimulation significantly increased production of IL-12p70 in TLR2 $2^{-/-}$ and TLR2 $/ 9^{-/-}$mice (Figure 3-8), which suggests TLR2 stimulation suppresses IL-12p70

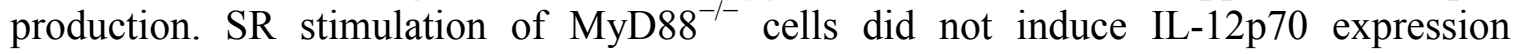
suggesting a MyD88-dependent receptor is necessary for the induction IL-12p70 protein expression. 

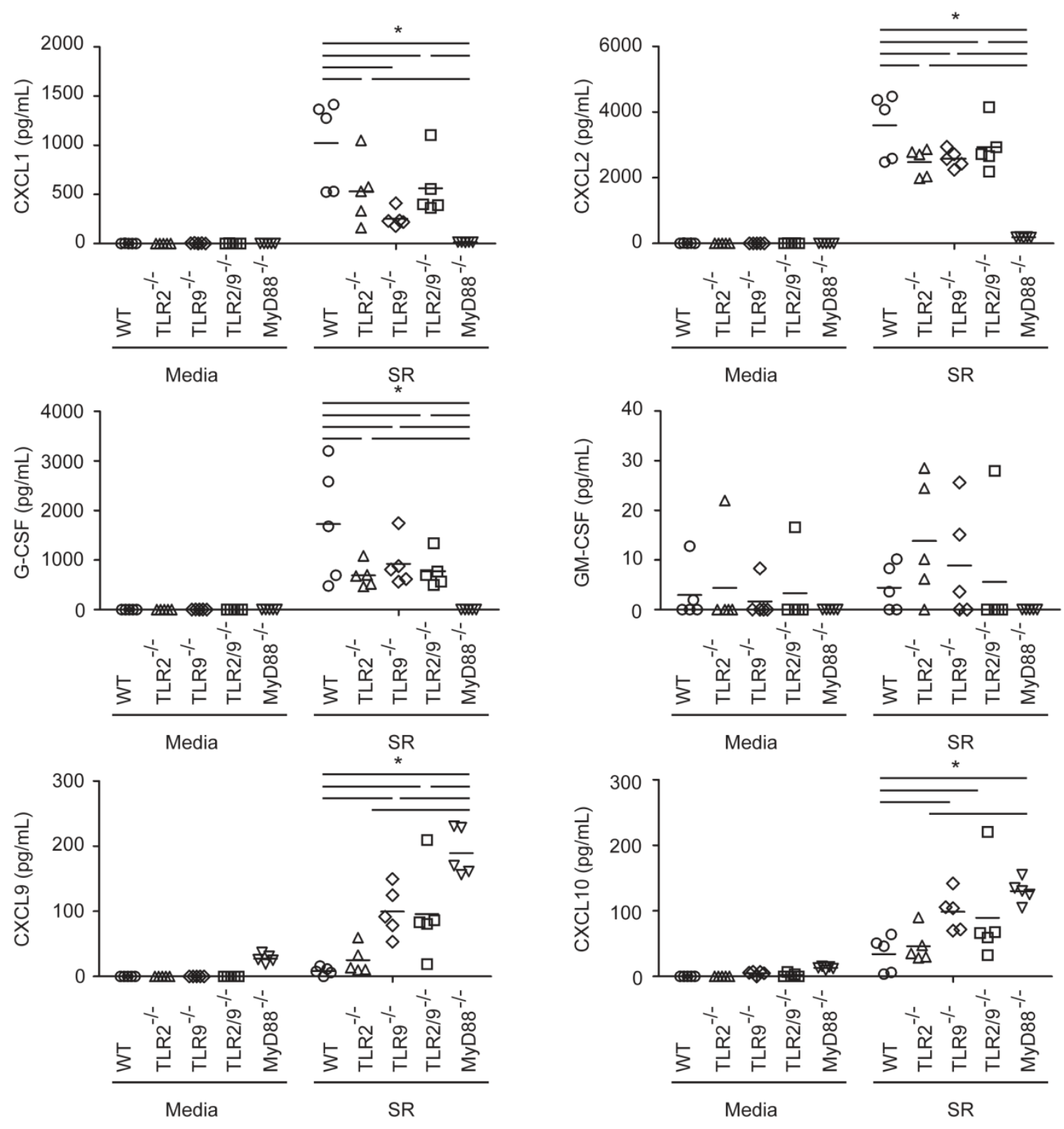

Figure 3-7. Chemokine production in splenic adherent cells stimulated with SR in vitro.

WT, TLR2 ${ }^{-/}, \mathrm{TLR}^{-/-}, \mathrm{TLR} 2 / 9^{-/-}$, and $\mathrm{MyD} 88^{-/-}$splenic adherent cells were plated at $5 \times 10^{5}$ cells per well and stimulated with media or SR $(0.05 \mathrm{mg} / \mathrm{mL})$ for 24 hours. Cell culture supernatants were collected and the expression of CXCL1, CXCL2, CXCL9, CXCL10, G-CSF, and GM-CSF was determined by Milliplex bead based ELISA (limit of detection $3.2 \mathrm{pg} / \mathrm{mL})$. Data represent the mean $\pm \mathrm{SD}(\mathrm{n}=5$ wells per group) and significance was determined using two-way ANOVA with Bonferroni post-hoc test (SR stimulation vs. genotype) $\left({ }^{*} p<0.05\right.$ compared to WT SR exposed cells or MyD88 ${ }^{-/-}$SR exposed cells). 
Figure 3-8. Cytokine production in splenic adherent cells stimulated with SR in vitro.

WT, TLR2 ${ }^{-/}$, TLR9 ${ }^{-/-}, \mathrm{TLR} 2 / 9^{-/-}$, and MyD88 ${ }^{-/-}$splenic adherent cells were stimulated with media or SR $(0.05 \mathrm{mg} / \mathrm{mL})$ for 24 hours. Cell culture supernatants were collected and the expression of TNF $\alpha$, IL-6, IL-1 $\beta$, IL-10, IL-12p70, IFN $\gamma$, and IL-17 was determined by Milliplex bead based ELISA. Data represent the mean $\pm \mathrm{SD}(\mathrm{n}=5$ wells per group) and significance was determined using two-way ANOVA with Bonferroni post-hoc test (SR stimulation vs. genotype) $\left({ }^{*} p<0.05\right.$ compared to WT SR exposed cells or MyD88 ${ }^{-/-}$ SR exposed cells). 

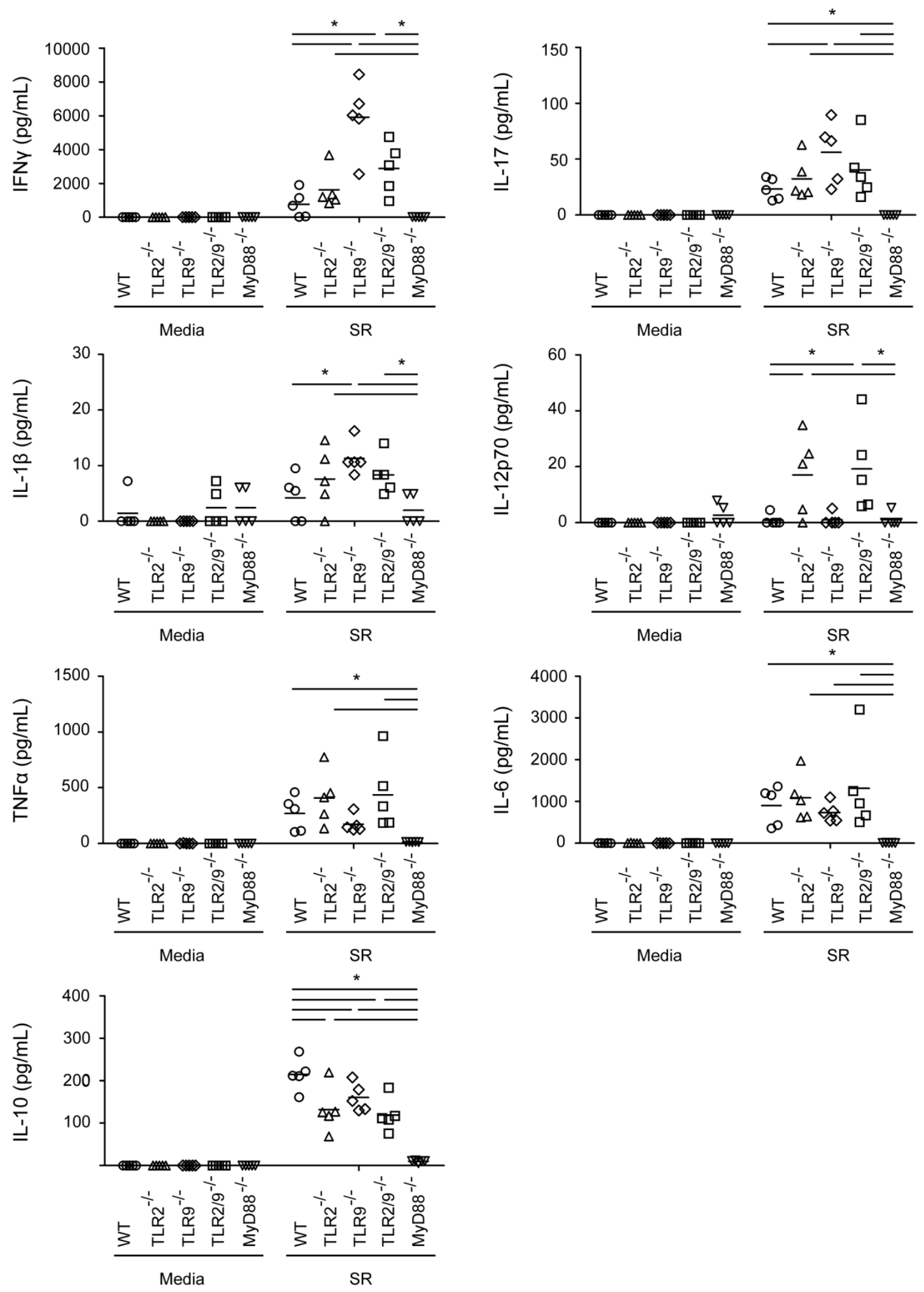
The inflammatory cytokines TNF $\alpha$ and IL- 6 are induced in response to SR stimulation in WT, TLR2 $2^{-/-}$, TLR $9^{-/-}$, and TLR2 $/ 9^{-/-}$cells, but significantly reduced in MyD88 $8^{-/-}$cells (Figure 3-8), suggesting a MyD88-dependent receptor other than TLR2 and TLR9 can induce TNF $\alpha$ and IL-6 expression in splenic adherent cells. The antiinflammatory cytokine IL-10 is significantly reduced in SKO, and TLR2/9 ${ }^{-/}$cells (Figure 3-8), suggesting IL-10 expression is partially dependent on TLR2 and TLR9. However, MyD88 ${ }^{-1-}$ cells have significantly less IL-10 than WT and KO cells suggesting another MyD88-dependent receptor is responsible for IL-10 expression in response to SR. Altogether, the results suggest there is an additional MyD88-dependent receptor involved in SR recognition leading to the production of CXCL1, CXCL2, G-CSF, IFN $\gamma$, IL-17, IL-1 $\beta$, IL-12p70, TNF $\alpha$, IL-6, and IL-10 in WT and TLR2/ $9^{-/-}$splenic adherent cells.

\section{TLR7 in SR Induced Cytokine Production In Vitro}

MyD88-dependent receptors include the IL-1/IL-18R and TLRs. To investigate whether the additional MyD88-dependent receptor involved in the recognition of SR was another TLR, we looked at TLR expression in the lung tissue after 3 weeks exposure to SR. We found increased expression of TLR7, a MyD88-dependent receptor that recognizes single stranded RNA (Figure 3-9). There was no increase in the expression of TLR3, TLR4, or TLR5 in the lungs after 3 weeks SR exposure, suggesting TLR3, TLR4, and TLR5 are not required for SR recognition.

To confirm that SR RNA is able to induce cytokine production, we stimulated WT and TLR2 $/ 9^{-1-}$ spleen cells with SR RNA complexed with DOTAP, a liposomal transfection agent (Figure 3-10A). SR RNA was able to stimulate TNF $\alpha$ production in WT and TLR2 $/ 9^{-/-}$spleen cells in a dose dependent manner. Non-MyD88-dependent cytoplasmic PRRs, such as RIG-I, can recognize RNA. To verify SR RNA stimulates spleen cells through a MyD88-dependent receptor, WT, TLR2 $/ 9^{-/-}$, and MyD88 ${ }^{-/-}$spleen cells were stimulated with SR RNA and IL-10 production measured (Figure 3-10B). SR RNA stimulated IL-10 production in WT and TLR2 $/ 9^{-/-}$spleen cells. There was a significant reduction in IL-10 in $\mathrm{MyD}^{-/-}$spleen cells stimulated with SR RNA

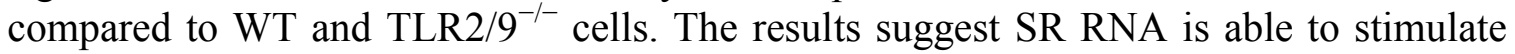
IL-10 production through a MyD88-dependent receptor such as TLR7.

TLR7 is present in endosomes and along with TLRs 3 and 9, and requires endosomal acidification for proteolytic cleavage of the receptor for activation (Ewald et al., 2008; Qi et al., 2012). Bafilomycin is an inhibitor of the vacuolar type $\mathrm{H}^{+}$ATPase, which inhibits endosomal acidification (Yoshimori et al., 1991); therefore, we hypothesized if an endosomal TLR was responsible for cytokine production in TLR2/9 ${ }^{-/-}$ cells, then cytokine production would be blocked by bafilomycin. We stimulated spleen cells from WT and TLR2 $/ 9^{-1-}$ mice with SR in the presence or absence of bafilomycin (Figure 3-11). The production of IL-6 and TNF $\alpha$ was significantly increased in WT SR stimulated cells compared to unexposed cells. The presence of bafilomycin did not 


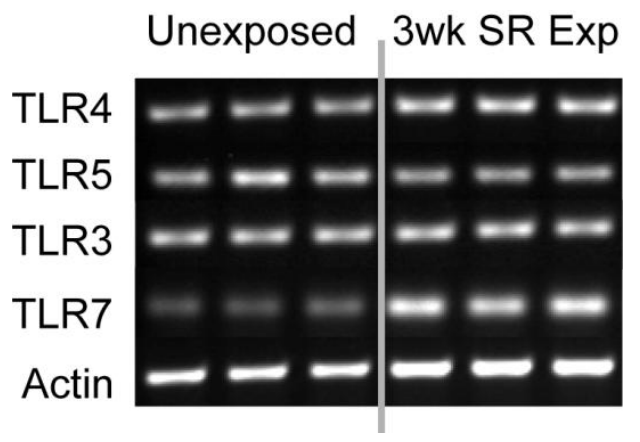

Figure 3-9. TLR7 expression is increased in mice exposed to SR for 3 weeks.

C57Bl/6 mice were exposed to SR 3 times/week for 3 weeks and sacrificed 12 hours after the last exposure. Lung tissue was harvested and RNA isolated for reverse transcription and PCR. TLR4, TLR5, TLR3, and TLR7 expression was measured by RT-PCR. Actin expression was used as a housekeeping gene control. 

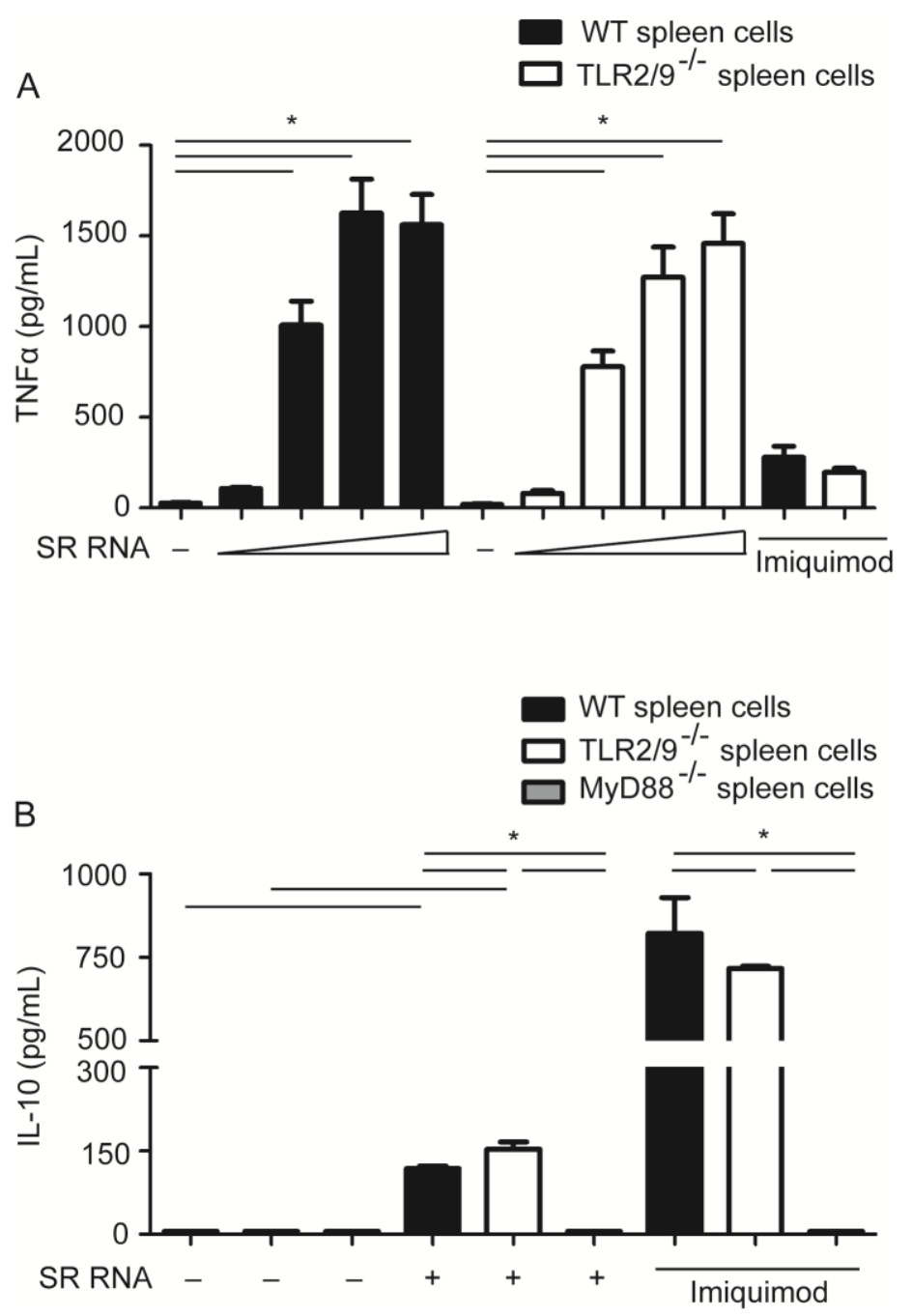

Figure 3-10. SR RNA stimulates cytokine expression in spleen cells in vitro.

(A) WT and TLR2 $/ 9^{-1-}$ spleen cells were stimulated with SR RNA complexed with DOTAP $(0.01,0.1,1.0$, and $10 \mu \mathrm{g} / \mathrm{mL})$. Imiquimod $(0.25 \mu \mathrm{g} / \mathrm{mL})$, a synthetic TLR7 agonist, was used as a positive control. Cell culture supernatants were collected and the expression of TNF $\alpha$ measured by ELISA. Data represent the mean \pm SD $(n=4$ wells per group) significance was determined using one-way ANOVA with Tukey post-hoc test

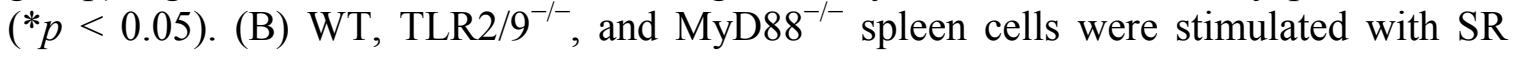
RNA complexed with DOTAP $(1 \mu \mathrm{g} / \mathrm{mL})$. Imiquimod $(0.25 \mu \mathrm{g} / \mathrm{mL})$, a synthetic TLR7 agonist, was used as a positive control. Data represent the mean \pm SD $(n=4$ wells per group) and significance was determined using one-way ANOVA with Tukey post-hoc test $(* p<0.05)$. 


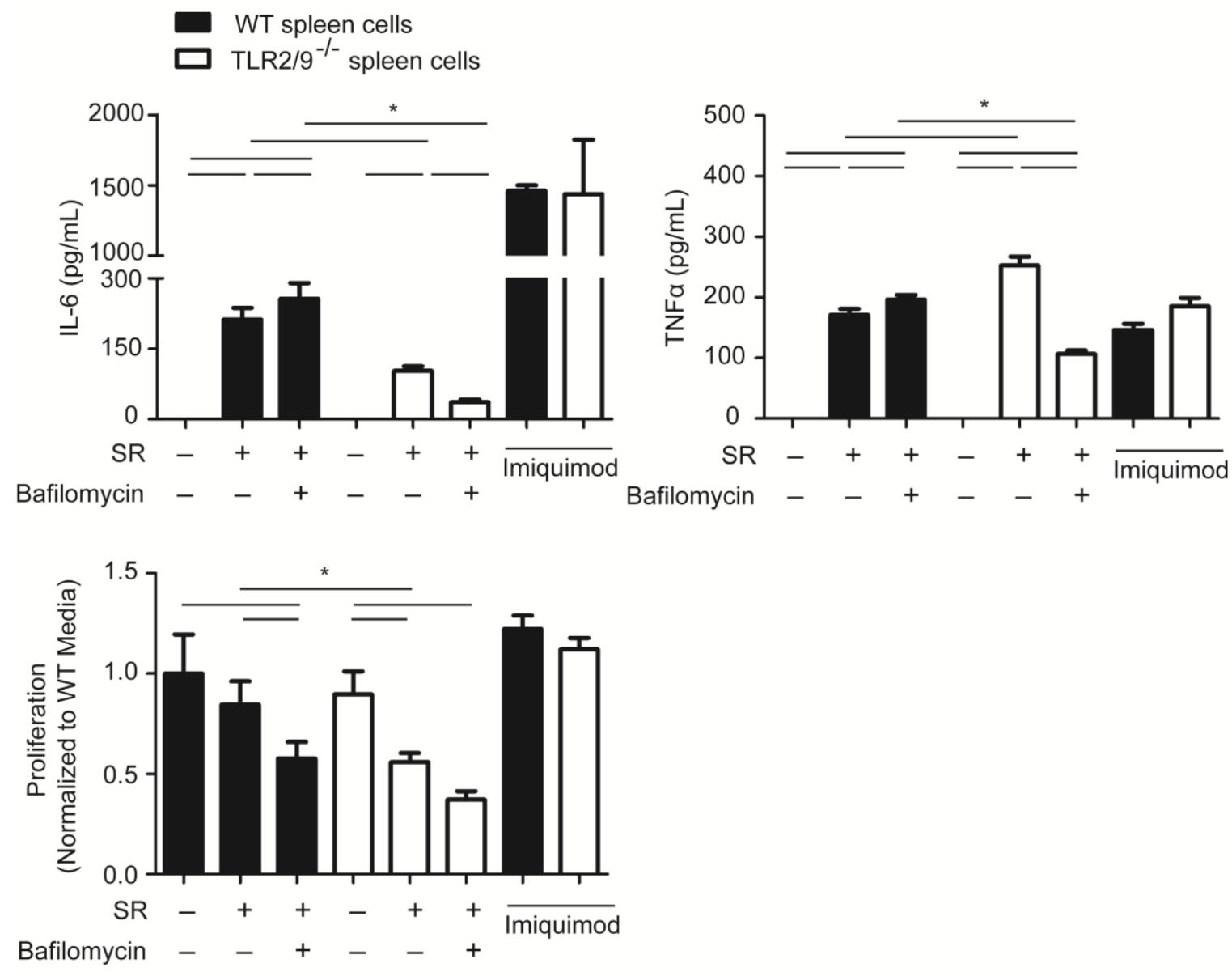

Figure 3-11. Bafilomycin inhibits IL-6 and TNFa production in TLR2/9 ${ }^{-/}$spleen cells stimulated with $\mathrm{SR}$ in vitro.

WT and TLR2 $/ 9^{--}$spleen cells were stimulated with media or SR $(0.025 \mathrm{mg} / \mathrm{mL})$ in the presence of absence of bafilomycin $(0.1 \mu \mathrm{M})$, an inhibitor of endosomal acidification, for 24 hours. Imiquimod $(0.25 \mu \mathrm{g} / \mathrm{mL})$, a synthetic TLR7 agonist, was used as a positive control. Cell culture supernatants were collected and the expression of TNF $\alpha$ and IL-6 measured by ELISA. Bafilomycin toxicity was assessed by Promega MTS cell proliferation assay. Data represent the mean $\pm \mathrm{SD}(\mathrm{n}=4$ wells per group $)$ and significance was determined using one-way ANOVA with Tukey post-hoc test $\left({ }^{*} p<\right.$ $0.05)$. 
significantly decrease IL-6 or TNF $\alpha$ production in WT cells. The production of IL-6 was significantly decreased, and the production of TNF $\alpha$ was significantly increased in TLR2 $/ 9^{-/-}$cells stimulated with SR compared to WT SR stimulated cells. The addition of bafilomycin to SR stimulated TLR2/ $9^{-/}$spleen cells significantly decreased the production of IL-6 and TNF $\alpha$ compared to SR alone. The reduction in TNF $\alpha$ and IL-6 production was not due to bafilomycin toxicity as there was no significant difference in proliferation between SR and SR + bafilomycin stimulated TLR2/9 ${ }^{-/}$spleen cells. Altogether, the results suggest an endosomal TLR contributes to IL-6 and TNF $\alpha$ production in SR stimulated spleen cells.

Macrophages are one of the first cell types in the lung to encounter SR upon exposure. To investigate SR stimulation of TNF $\alpha$ and IL-6 production in macrophages, BMDMs were differentiated from bone marrow cells for 7 days in the presence of LCCM. RNA was isolated from WT and TLR $2 / 9^{-/}$BMDMs for RT-PCR to verify BMDMs express TLR7 (Figure 3-12A). WT BMDMs stimulated with SR express IL-6 and $\mathrm{TNF} \alpha$, but there was no significant decrease in expression in the presence of bafilomycin (Figure 3-12B). TLR2/ $/ 9^{-/}$SR stimulated BMDMs express significantly less IL-6 and TNF $\alpha$ than WT SR stimulated BMDMs, but the addition of bafilomycin did not significantly decrease IL-6 and TNF $\alpha$ production compared to TLR2/9 $/{ }^{-/}$SR stimulated BMDMs. Imiquimod stimulated IL-6 and TNF $\alpha$ production in WT and TLR2/9 $9^{-/}$ BMDMs, suggesting TLR7 is functional in these cells. Bafilomycin was able to significantly reduce the production of IL-6 and TNF $\alpha$ in imiquimod stimulated cells, suggesting the concentration of bafilomycin used in the experiment was able to suppress TLR7 induced cytokine production. Altogether, the results suggest TNF $\alpha$ and IL-6 production in TLR2/ $/ 9^{-/}$BMDMs is not induced by an endosomal TLR, such as TLR7.

\section{Discussion}

SR is a strong inducer of neutrophil chemokines and neutrophils are rapidly recruited to the lungs after a single exposure to SR. The recruitment of neutrophils to the lungs is dependent on TLRs 2 and 9 as evidenced by the significant reduction in neutrophils recruited to the lungs of TLR $2 / 9^{-/-}$mice. The reduced neutrophil recruitment can partially be explained by reduced neutrophil chemokine production in TLR2/9 $9^{-/}$ mice compared to WT; however, neutrophil chemokines were also significantly reduced in $\mathrm{TLR}^{-/-}$, and $\mathrm{TLR}^{-/-}$mice compared to WT exposed mice. Inflammatory cytokines also play a role in neutrophil recruitment through the upregulation of selectins and cell adhesion molecules on endothelial cells (Wagner and Roth, 2000). TNF $\alpha$ was significantly reduced in the BALF of TLR2 $2^{-/}$and TLR $2 / 9^{-/-}$mice, and IL-6 was significantly reduced in TLR $2 / 9^{-/}$mice compared to WT exposed mice. SR stimulation also induces the production of monocyte/lymphocyte chemokines CCL3/MIP-1 $\alpha$ and CCL4/MIP-1 $\beta$. CCL3 production was significantly reduced in all KO mice, whereas CCL4 was only significantly reduced in TLR $2 / 9^{-/-}$mice suggesting TLRs 2 and 9 both contribute to the production of CCL3 and CCL4. Altogether the results suggest SR stimulation through TLRs 2 and 9 is important for the recruitment of neutrophils in HP, 

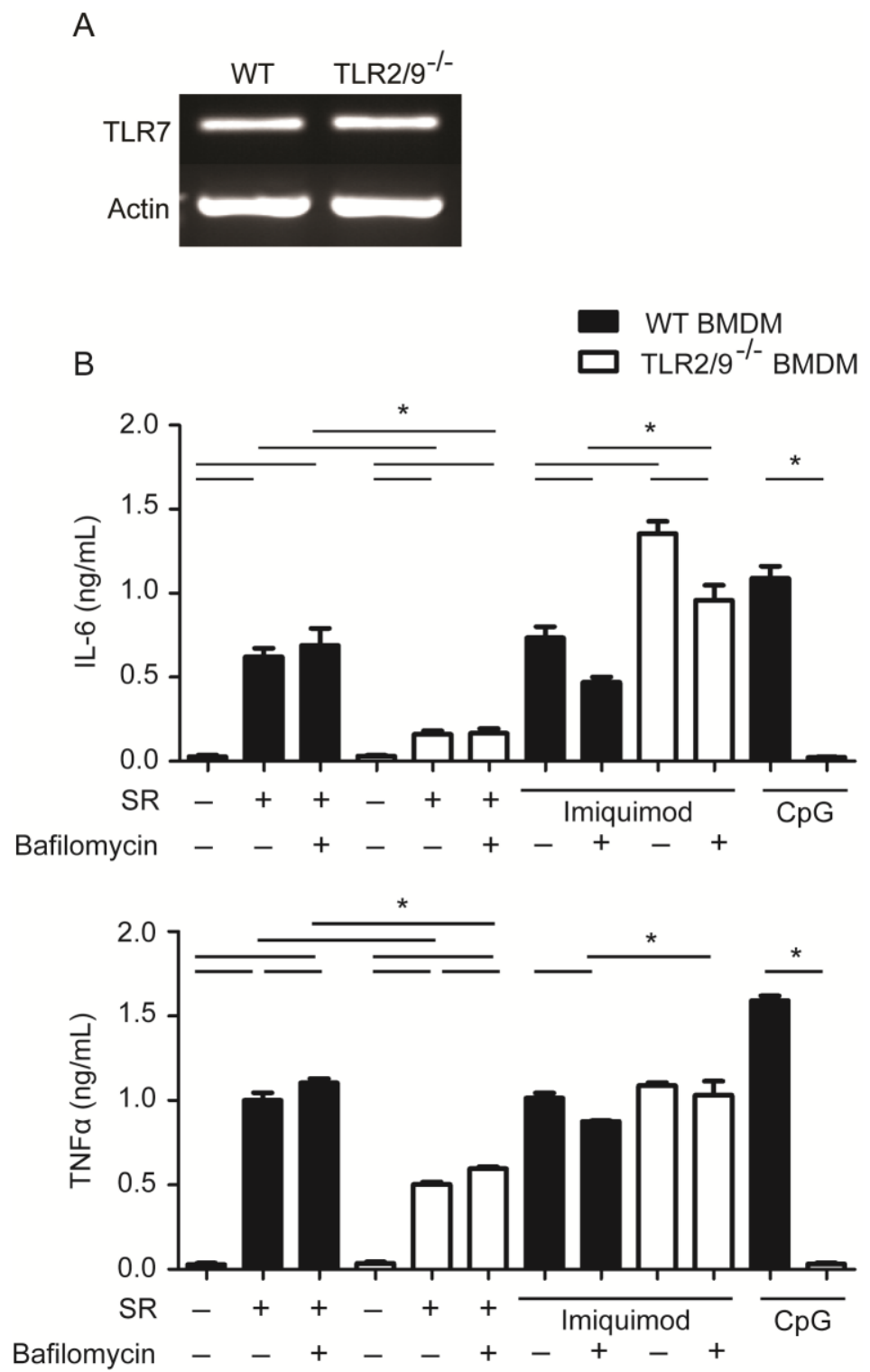

Figure 3-12. Bafilomycin does not inhibit IL-6 and TNFa in BMDM stimulated with $S R$ in vitro.

(A) RNA was isolated from BMDMs for reverse transcription and PCR. TLR7 expression was analyzed. Actin expression was used as a housekeeping gene control. (B) WT and TLR2 $/ 9^{-1-}$ BMDMs were stimulated with media or SR $(0.05 \mathrm{mg} / \mathrm{mL})$ in the presence or absence of bafilomycin $(0.1 \mu \mathrm{M})$, an inhibitor of endosomal acidification, for 24 hours. Imiquimod $(3 \mu \mathrm{g} / \mathrm{mL})$, a synthetic TLR7 agonist, in the presence or absence of bafilomycin was used as a positive control of TLR7 inhibition by bafilomycin. CpG, a TLR9 agonist, was used as a positive control. Cell culture supernatants were collected and the expression of TNF $\alpha$ and IL-6 measured by ELISA. 
and may also contribute to monocyte and lymphocyte recruitment to the lung at later time points.

SR exposed mice had increased the production of IL-12p40 in the BALF. IL-12p40 is the common chain for the cytokines IL-12p70 (IL-12p35 / IL-12p40) and IL-23 (IL-23p19 / IL-12p40), two cytokines important in the polarization and proliferation of Th1 and Th17 cells. There was no IL-12p70 detected in the BALF suggesting SR induces the production of IL-23, and IL-23p19 expression was increased in SR exposed mice. SR induced expression of IL-12p40 and IL-23p19 was largely dependent on TLR2, as TLR2 ${ }^{-/-}$and $\mathrm{TLR} 2 / 9^{-/-}$mice had a significant reduction in expression compared to WT exposed mice; however, only TLR $2 / 9^{-1-}$ mice had a significant reduction in IL-12p40 in the BALF. IL-23 is a cytokine that is important for the proliferation and survival of Th17 cells, which have been associated with disease severity and fibrosis in HP. Therefore, TLR $2 / 9^{-/-}$mice may be protected from Th17 cell associated pathogenesis at later time points. The expression of the anti-inflammatory cytokine IL-10 was also upregulated in response to SR, and the upregulation was dependent on TLR2. However, IL-10 was not detected in the BALF at this time point. Reduced anti-inflammatory cytokine production could have a negative impact on disease progression in $\mathrm{TLR}^{-/-}$and TLR2 $/ 9^{-/-}$mice at later time points.

Although TLR2 $/ 9^{--}$mice had reduced neutrophil recruitment, inflammatory cytokine and chemokine production after one exposure to SR, the levels were still greater than previous studies with MyD88 $8^{-/}$mice (Nance et al., 2008). In vitro stimulation of splenic adherent cells with SR revealed another MyD88-dependent receptor could be involved in the residual production of CXCL1, CXCL2, G-CSF, TNF $\alpha$, IL-6, IL-1 $\beta$,

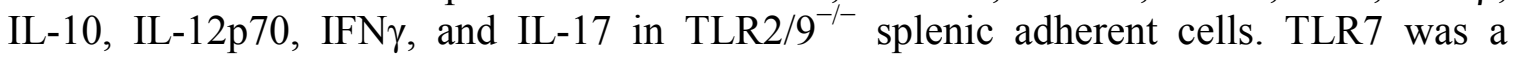
possible candidate since it was upregulated in the lungs of mice exposed to SR for 3 weeks. TLR7 recognizes single stranded RNA, and SR RNA stimulated the production of TNF $\alpha$ in a dose dependent manner, and the production of IL-10 through a MyD88dependent receptor. Inhibition of endosomal TLRs with bafilomycin showed a significant reduction of TNF $\alpha$ and IL-6 production in TLR2/ $/ 9^{-1-}$ spleen cells, but not BMDMs. RTPCR revealed BMDMs express TLR7, and stimulation with the TLR7 agonist imiquimod suggests the TLR7 receptor is functional and that bafilomycin can inhibit TLR7 induced TNF $\alpha$ and IL-6 production in BMDMs. Altogether, the results suggest an endosomal TLR such as TLR7 plays a role in SR induced TNF $\alpha$ and IL-6 production in the spleen, but not in BMDMs. TLR13 is an endosomal TLR, which has recently been shown to recognize bacterial 23S rRNA in mice (Oldenburg et al., 2012). TLR13 is not expressed in humans and therefore would not contribute to SR induced HP in patients. The IL-1R and IL-18R is MyD88-dependent and may play a role in SR induced inflammatory cytokine production in BMDMs.

In summary, TLR $2 / 9^{-/-}$mice have significant reduction in the recruitment of neutrophils, a cell type that is important in innate immune defense against bacteria, but is associated with increased inflammation and lung pathology in HP. TLR2/9 ${ }^{-/}$also have significantly reduced inflammatory cytokine production after SR exposure. Importantly, TLR2 $/ 9^{-/-}$mice have significant reductions in IL-6 and IL-23p19, two cytokines 
important for the differentiation and proliferation of Th17 cells, which have been associated with lung fibrosis in HP. In the next chapter, we will explore the role of TLRs 2 and 9 in neutrophil recruitment, macrophage and lymphocyte activation, polarized Th cell phenotypes, and granuloma formation after three weeks repeated SR exposure. 


\section{CHAPTER 4. GRANULOMATOUS PHASE ${ }^{*}$}

\section{Introduction}

The granulomatous phase of HP is characterized by lymphocytic alveolitis and granuloma formation. Previous studies have revealed HP is a T cell mediated disease. Nude mice, which lack a thymus and do not develop mature T cells, do not develop HP (Ohta et al., 1993), and TcR $\beta^{-/}$mice, which lack $\mathrm{CD} 4^{+}$and $\mathrm{CD} 8^{+} \mathrm{T}$ cells, do not form granulomas or develop severe fibrosis in response to SR (Simonian et al., 2009). Granuloma formation depends on interactions between macrophages and $\mathrm{T}$ cells through cytokine stimulation and receptor interactions. In HP, the cytokine IFN $\gamma$ produced from T cells, neutrophils, or other innate immune cells is required for granuloma formation (Gudmundsson and Hunninghake, 1997; Nance et al., 2005). The activation of antigen specific Th cells is also critical for granuloma formation. TLR stimulation of antigen presenting cells leads to the upregulation of costimulatory molecules CD80 and CD86, increased expression of MHCII, and induces cytokine secretion necessary for the activation and polarization of naïve Th cells into effector cells (Pasare and Medzhitov, 2003; Pasare and Medzhitov, 2004). In addition, costimulatory molecules CD80 and CD86, CD40, and MHCII have been shown to be upregulated on macrophages inside granulomas in tuberculosis, schistosomiasis, and Crohn's disease (Hara et al., 1997; Rathore et al., 1996; Soler et al., 1999). Studies during the acute phase revealed the importance of TLRs 2 and 9 in neutrophil recruitment, neutrophil chemokine production, and inflammatory cytokine production in response to a single SR exposure. During the granulomatous phase, we hypothesize TLRs 2 and 9 cooperate in neutrophil recruitment as well as $\mathrm{T}$ cell activation and costimulatory molecule upregulation necessary for granuloma formation and the pathogenic $\mathrm{T}$ cell response.

\section{T Cell Activation}

Naïve $\mathrm{CD} 4^{+} \mathrm{T}$ cells require 3 signals for activation. The first signal comes from the $\mathrm{T}$ cells receptor, which recognizes antigen displayed on major histocompatibility complex class II (MHCII) on the cells surface of an antigen presenting cell (APC), such as a dendritic cell or macrophage. The second signal is transmitted through the binding of costimulatory molecules CD80 or CD86 to CD28 on the T cell. The third signal of activation is through cytokine stimulation released from the APC and binding to cytokine receptors on the $\mathrm{T}$ cell. The activated $\mathrm{Th}$ cell then matures and differentiates into $\mathrm{T}$

*Adapted with permission from Andrews K, Abdelsamed H, Yi A-K, Miller MA, Fitzpatrick EA (2013) TLR2 Regulates Neutrophil Recruitment and Cytokine Production with Minor Contributions from TLR9 during Hypersensitivity Pneumonitis. PLoS ONE 8(8): e73143. 
effector cells, which travel to sites of infection to augment the immune response, or the Th cell can differentiate into a memory cell, which populates lymphoid organs such as the spleen and lymph nodes. Effector and memory $\mathrm{T}$ cells only require 1 signal for activation, the $\mathrm{T}$ cell receptor interacting with antigen displayed on MHCII.

$\mathrm{CD}^{+}$Th cells polarize into different subtypes depending on their cytokine stimulation (reviewed in Weaver et al., 2007). Th cells exposed to IL-12p70 and IFN $\gamma$ upregulate the transcription factor T-bet, which leads to polarization of Th1 cells. Exposure to IL-4 and the upregulation of the transcription factor Gata3 induces Th2 cell polarization. Finally, in the mouse, TGF $\beta$ in the presence of IL- 6 upregulates the transcription factor ROR $\gamma$ and polarizes Th17 cells, while TGF $\beta$ alone polarizes $\mathrm{T}$ regulatory (Treg) cells and induces the upregulation of the transcription factor FoxP3. The Th17 response in SR exposed mice is associated with increased disease severity and fibrosis (Joshi et al., 2009; Simonian et al., 2009). Previous studies have shown that $\mathrm{TLR}^{-/-}$mice have significantly decreased expression of IL-6 and IL-17, cytokines associated with the Th17 response, suggesting SR stimulation through the TLR2/6 heterodimer is involved in IL-17 expression and the Th17 response (Fong et al., 2010). During the acute phase, signaling through TLR2 was revealed to be important in SR induced production of IL-6 and IL-23p19 expression, suggesting polarization of the Th17 response may be inhibited in TLR2 $2^{-/}$and TLR $2 / 9^{-/-}$mice.

\section{TLR Induced Macrophage Activation}

Like $\mathrm{T}$ cells, macrophages can exhibit polarized phenotypes depending on the chemokines and stimuli in their environment (reviewed in Mosser, 2003; Mosser and Edwards, 2008). Stimulation of TLRs on macrophages in the presence of IFN $\gamma$ and TNF $\alpha$ leads to the classically activated phenotype characterized by upregulation of nitric oxide synthase, costimulatory molecules CD80 and CD86, and MHCII. Nitric oxide synthase generates nitric oxide, which is a potent antimicrobial agent (Bogdan et al., 2000). Classically activated macrophages release inflammatory cytokines such as TNF $\alpha$, IL-12, IL-1, and IL-6. However, TLR signaling combined with immune complexes leads to a regulatory macrophage that also upregulates costimulatory molecules, MHCII, and nitric oxide production, but secretes low levels of IL-12 and high levels of IL-10 (Edwards et al., 2006), and are therefore important for dampening immune responses.

Activated effector $\mathrm{T}$ cells can recognize bacterial proteins displayed on the surface of macrophages on MHC molecules through the TcR. T cells are an important source of cytokines to induce macrophage activation through the secretion of cytokines such as IFN $\gamma$ or IL-10. Effector T cells also express CD40 ligand, which interacts with CD40 expressed on the surface of macrophages to send contact dependent activating signals to the macrophage (Stout et al., 1996). Macrophages are well known to upregulate costimulatory molecules in response to bacterial and TLR stimulation (Bashir et al., 2010; Dong et al., 2008; Shim et al., 2009). Alveolar macrophages (AMs) have low costimulatory molecule expression in a healthy lung, however in patients with HP, costimulatory molecules are upregulated (Israel-Assayag et al., 1999). 
In the following studies, the objective is to analyze neutrophil and lymphocyte recruitment, inflammatory cytokine and chemokine production, lymphocyte and macrophage activation, the Th17 response, and granuloma formation following repeated exposure to SR. We hypothesize the loss of TLRs 2 and 9 in SR exposed mice will result in reduced neutrophil recruitment due to reduced inflammatory cytokine and chemokine production during the granulomatous phase. In addition, we hypothesize TLRs 2 and 9 are required for a Th17 cell response through the induction of inflammatory cytokines IL-6 and IL-12p40 (the common chain for IL-12 and IL-23).

\section{Results}

In the following studies we measured disease severity through alveolitis, inflammatory cytokine and chemokine production, and granuloma formation. In the alveolitis section, we examined the composition of the alveolitis in WT, TLR2 ${ }^{-/}$, TLR9 ${ }^{-/-}$, and TLR2 $/ 9^{-/-}$mice exposed to SR for neutrophil and lymphocyte recruitment and markers of lymphocyte activation. In the granuloma section, we explored granuloma formation through the analysis of lymphocyte populations in the lung, the upregulation of lymphocyte activation markers and polarized Th cell subtypes, the upregulation of costimulatory molecules on macrophage populations in the lung, and the formation of granulomas by histology.

\section{Alveolitis}

\section{Neutrophil Recruitment and Chemokine Production Are Largely Dependent on TLR2 Following Repeated SR Exposures}

To determine whether TLRs 2 and 9 also control neutrophil recruitment throughout 3 weeks of repeated exposures, we exposed WT, TLR2 ${ }^{--}$, TLR9 ${ }^{-/}$, and TLR2 $/ 9^{-/-}$mice to SR 3 times/week for 3 weeks and performed BAL on day 18 (one day after the last exposure) and day 20 ( 3 days after the last exposure). The results demonstrate that there is no significant reduction in alveolitis in the TLR2/9 ${ }^{-/-}$mice compared to the WT mice on day 18 and day 20 (Figure 4-1A). To investigate whether the cellular composition of the BAL is altered in $\mathrm{KO}$ mice, immune cell populations were measured by flow cytometry. Figure 4-1B shows the gating strategy used to analyze the percentages of Gr-1 $1^{+} / \mathrm{CD} 11 \mathrm{~b}^{+}$neutrophils, Gr- $1^{+} / \mathrm{CD} 11 \mathrm{~b}^{-}$cells, and $\mathrm{CD} 11 \mathrm{~b}^{+} / \mathrm{Gr}-1^{-}$ monocytes present in the BAL cells. The results show the composition of the alveolitis is different during the granulomatous phase compared with an acute exposure. In WT mice, neutrophils were still the dominant cell type on day 18 but were decreased by day 20 . However, the TLR $2 / 9^{-/-}$mice still had a significant reduction of neutrophil frequency compared to the WT group on day 18 and day 20 (Figure 4-1C). The decrease in neutrophil recruitment to the airways appeared to be compensated for by a significant increase in $\mathrm{CD}_{11 b^{+}} / \mathrm{Gr}^{-} 1^{-}$cells. 
Figure 4-1. TLR2 is the dominant receptor in neutrophil recruitment to the airways of mice exposed to SR for 3 weeks.

WT, TLR2 ${ }^{-/-}$, $\mathrm{TLR}^{-/-}$, and TLR2 $/ 9^{-/-}$mice were exposed to SR 3 times/week for 3 weeks and analyzed on day 18 ( 1 day after the last exposure) or day 20 (3 days after the last exposure). (A) BAL was performed and alveolitis determined using trypan blue dye exclusion. Cells recovered from the BAL fluid were incubated with antibody to CD11b and Gr-1 and analyzed by flow cytometry. (B) The gating strategy to discriminate neutrophil $\left(\mathrm{CD} 11 \mathrm{~b}^{+} / \mathrm{Gr}-1^{\text {high }}\right), \mathrm{Gr}_{-} 1^{+}$cell $\left(\mathrm{Gr}-1^{+} / \mathrm{CD} 11 \mathrm{~b}^{-}\right)$, and monocyte $\left(\mathrm{Gr}-1^{-} /\right.$ $\mathrm{CD} 11 \mathrm{~b}^{+}$) populations present in the alveolitis. (C) The number of neutrophils, Gr- $1^{+}$cells, and monocytes in the BAL ( $n=3-4$ mice per group). The data represent mean $\pm \mathrm{SD}$ and significance was determined using one-way ANOVA with Tukey post-hoc test $\left({ }^{*} p<0.05\right.$ compared to WT SR exposed mice). 
Alveolitis

A

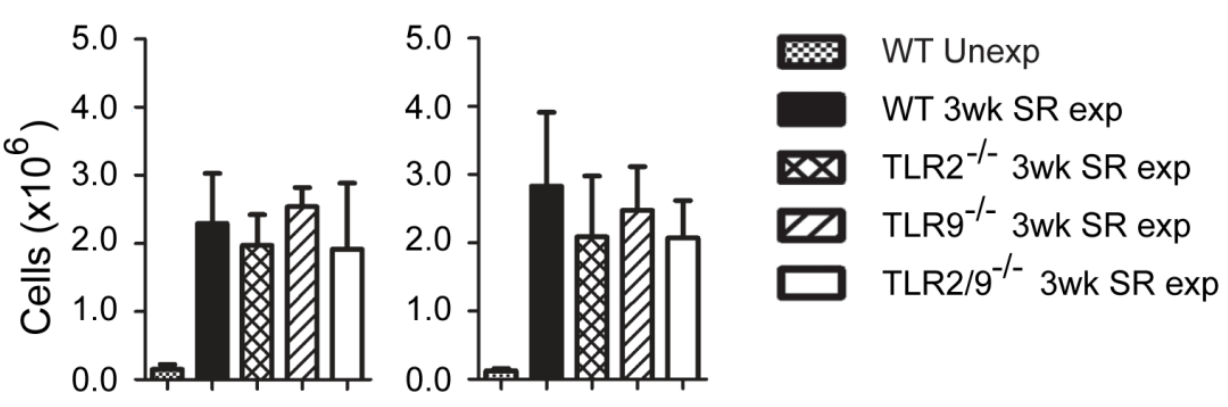

B

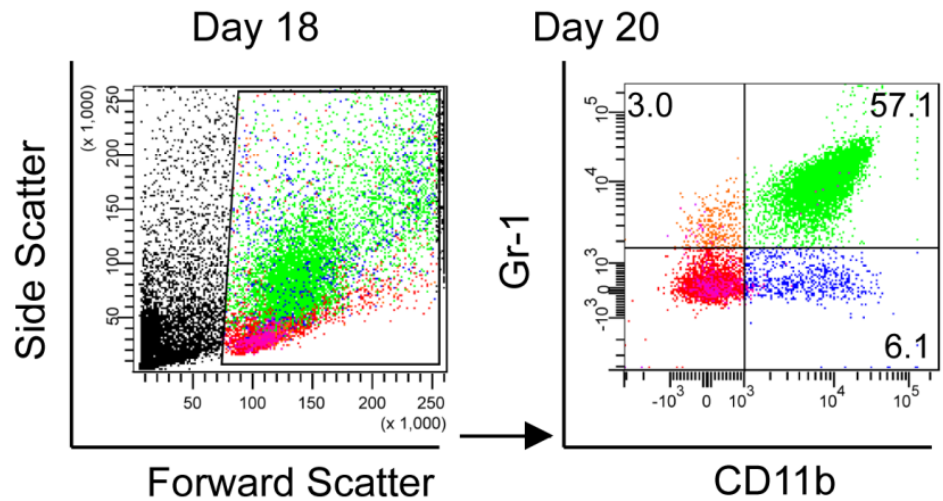

C

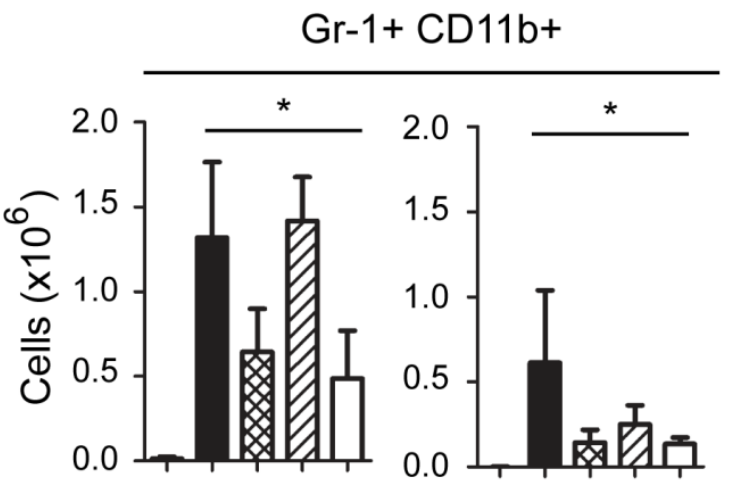

Day 18

Day 20

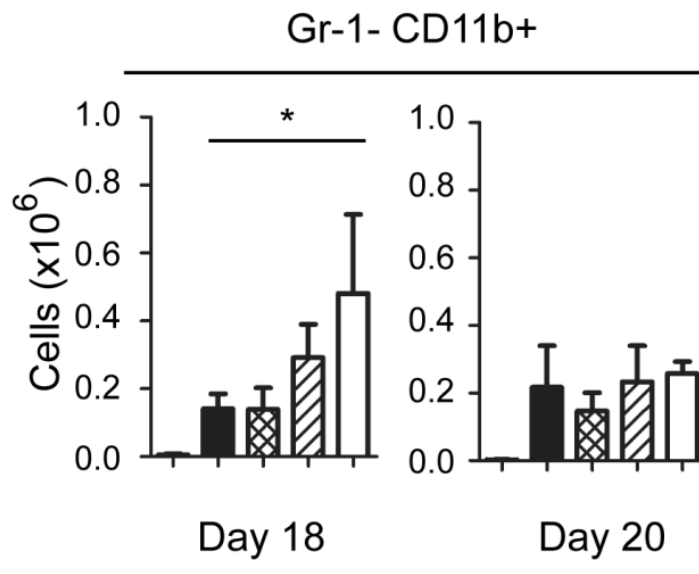

Gr-1+ CD11b-

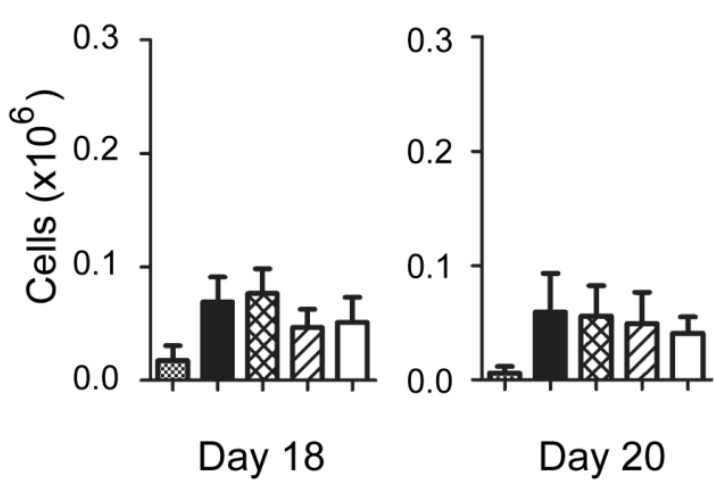


To determine if the reduced neutrophil recruitment in TLR $2 / 9^{-/-}$mice was due to impaired chemokine expression, RNA was isolated from the lungs of mice exposed to SR for 3 weeks for reverse transcription and RT-PCR. The expression of CXCL2 was significantly decreased in both the TLR2 ${ }^{-/-}$and TLR $2 / 9^{-/-}$mice compared to the WT mice (Figure 4-2). However, CXCL1 mRNA was not significantly reduced between the WT and mutant mice suggesting that TLRs 2 and 9 are not responsible for its production. The expression of macrophage and lymphocyte chemokines CCL2 and CCL4 was also upregulated after 3 weeks SR exposure, but the difference between WT and KO mice was not significant. Altogether, these results demonstrated that neutrophil recruitment and CXCL2 production following repeated SR exposures is largely dependent on TLR2.

\section{Lymphocytes Are Recruited to the Airways after 3 Weeks Exposure to SR}

After three weeks exposure to SR, lymphocytes were also present in greater numbers. The gating strategy for the detection of lymphocytes in the BAL is depicted in Figure 4-3A. Of the lymphocytes, $T$ cells and $B$ cells were more numerous than NK cells and $\gamma \delta \mathrm{T}$ cells, which is reflective of the adaptive immune response generated against SR (Figure 4-3B). On day 20, additional Th and B cells were recruited to the lung compared to day 18. However, there was no significant difference in the composition of lymphocytes in the BAL in single and double knockout mice.

To determine if the $\mathrm{T}$ cells recruited to the lung after 3 weeks exposure to SR were $\mathrm{CD}^{+} \mathrm{T}$ helper (Th) cells or $\mathrm{CD} 8^{+} \mathrm{T}$ cells, the percentage of $\mathrm{T}$ cells expressing $\mathrm{CD}^{+}$or $\mathrm{CD} 8^{+}$was analyzed by flow cytometry. Unexposed mice were excluded from the analysis as $\mathrm{T}$ cells are not present in the BAL of unexposed mice. The gating strategy to discriminate $\mathrm{CD}^{+}$and $\mathrm{CD} 8^{+} \mathrm{T}$ cell populations is depicted in Figure 4-4A. A greater percentage of $\mathrm{T}$ cells were $\mathrm{CD}^{+} \mathrm{Th}$ cells than $\mathrm{CD} 8^{+} \mathrm{T}$ cells in WT and $\mathrm{KO}$ mice (Figure 4-4B). $\mathrm{TLR}^{-/-}$mice had a significant decrease in the percentage of $\mathrm{CD} 8^{+} \mathrm{T}$ cells on day 18 . On day 20 , there was a greater percentage of $\mathrm{CD}^{+} \mathrm{T}$ cells than day 18 , which resulted in an increased CD4:CD8 ratio (Figure 4-4C) and suggests a greater number of $\mathrm{CD}^{+}$Th cells rather than $\mathrm{CD} 8^{+} \mathrm{T}$ cells are recruited to the airways at this time point.

\section{TLR2 Is Important for CD4 ${ }^{+}$Th Cell Activation, While TLR9 Is Important for $\mathrm{CD8}^{+} \mathrm{T}$ Cell Activation in the Airways}

To determine if TLRs 2 and 9 are necessary for lymphocyte activation, the percentage of lymphocytes expressing CD69, a marker of lymphocyte activation, was analyzed on T cells, B cells, NK cells, and $\gamma \delta \mathrm{T}$ cells. Unexposed mice were excluded from the analysis as T cells, B cells, NK cells, and $\gamma \delta \mathrm{T}$ cells are not present in the BAL of unexposed mice. In WT SR exposed mice, more than half of the $\mathrm{CD} 4^{+}$and $\mathrm{CD} 8^{+} \mathrm{T}$ cells express CD69 on day 18. TLR2 ${ }^{--}$mice had a reduced percentage of $\mathrm{CD} 69^{+} \mathrm{CD} 4^{+} \mathrm{Th}$ cells on day 18 , and TLR2 $2^{-/}$and TLR2 $/ 9^{-/-}$had significantly reduced percentage of $\mathrm{CD} 9^{+} \mathrm{CD}^{+}$Th cells on day 20 (Figure 4-5), suggesting signaling through TLR2 is 

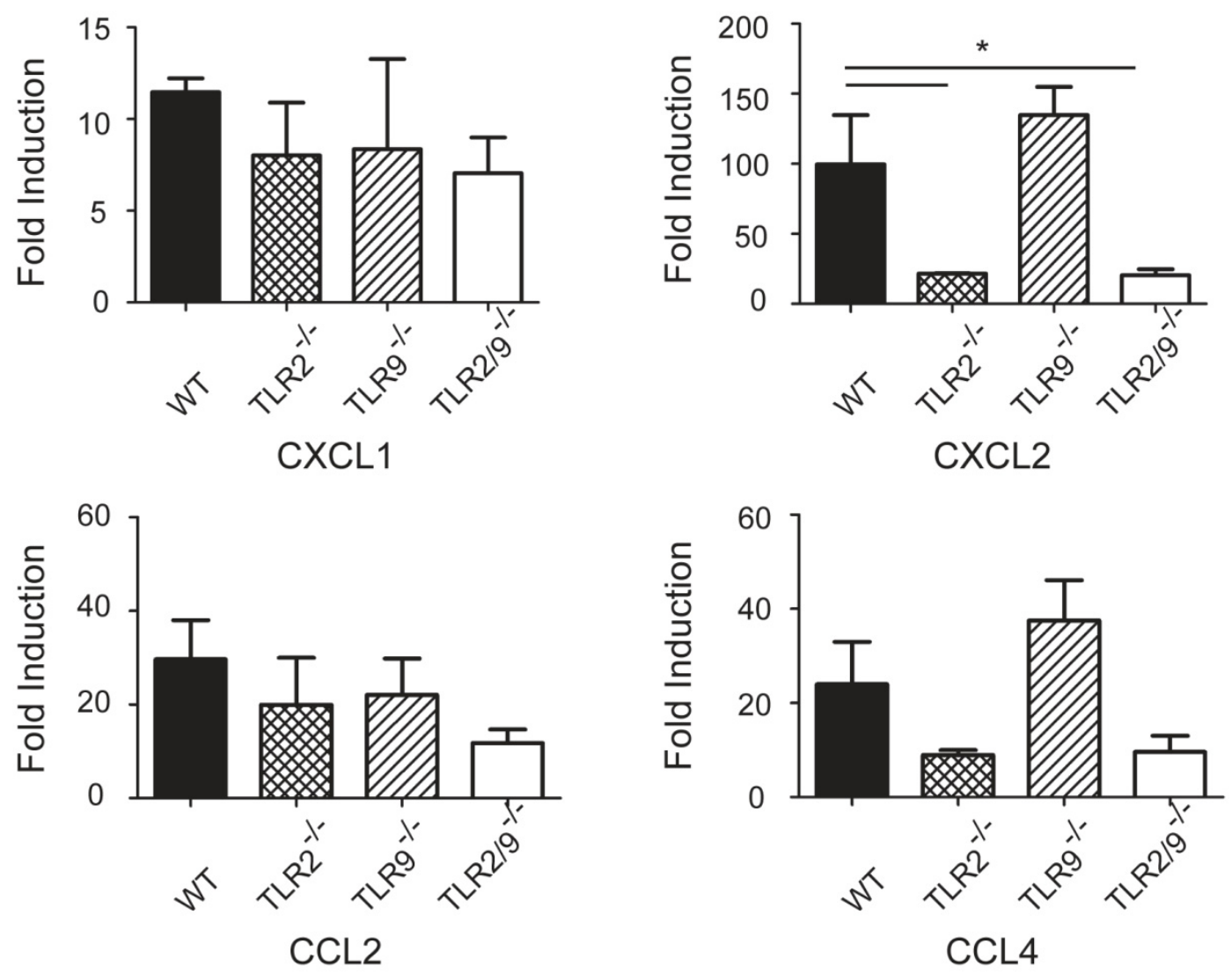

Figure 4-2. Neutrophil chemokine expression is dependent on TLR2 following 3 weeks SR exposure.

WT, TLR2 ${ }^{-/-}$, $\mathrm{TLR}^{-/-}$, and TLR2 $/ 9^{-/-}$mice were exposed to SR 3 times/week for 3 weeks and analyzed on day 18 ( 1 day after the last exposure). The expression of chemokines was determined by qRT-PCR on RNA isolated from individual lung lobes ( $n$ $=3$ mice per group) and is expressed as fold induction over WT unexposed mice. The data represent mean $\pm \mathrm{SD}$ of duplicate samples. Data represent the mean $\pm \mathrm{SD}(\mathrm{n}=3$ mice / group). Significance was determined using one-way ANOVA with Tukey post-hoc test $(* p<0.05$ compared to WT SR exposed mice). 
Figure 4-3. Mice exposed to SR for 3 weeks have increased leukocyte populations in the BAL.

WT, TLR2 ${ }^{-/-}$, $\mathrm{TLR}^{-/-}$, and TLR2 $/ 9^{-/-}$mice were exposed to SR 3 times/week for 3 weeks and analyzed on day 18 (1 day after the last exposure) or day 20 (3 days after the last exposure). BAL was performed and cells recovered from the BAL fluid were incubated with antibody to CD19, $\beta \mathrm{TcR}, \mathrm{NK} 1.1$, and $\gamma \delta \mathrm{TcR}$ and analyzed by flow cytometry. (A) The gating strategy to discriminate B cell $\left(\mathrm{CD} 19^{+} / \mathrm{SSc}^{\text {low }}\right)$, Th cell $\left(\beta \mathrm{TcR}^{+} / \mathrm{SSc}^{\text {low }}\right), \mathrm{NK}$ cell $\left(\mathrm{NK} 1.1^{+} / \mathrm{SSc}^{\text {low }}\right)$, and $\gamma \delta \mathrm{T}$ cell $\left(\gamma \delta \mathrm{TcR}^{+} / \mathrm{SSc}^{\text {low }}\right)$ populations present in the alveolitis. (B) The number of, T cells, B cells, NK cells, and $\gamma \delta \mathrm{T}$ cells present in the BAL. ( $n=3-4$ mice per group). The data represent mean $\pm \mathrm{SD}$ and significance was determined using one-way ANOVA with Tukey post-hoc test $\left({ }^{*} p<0.05\right.$ compared to WT SR exposed mice). 


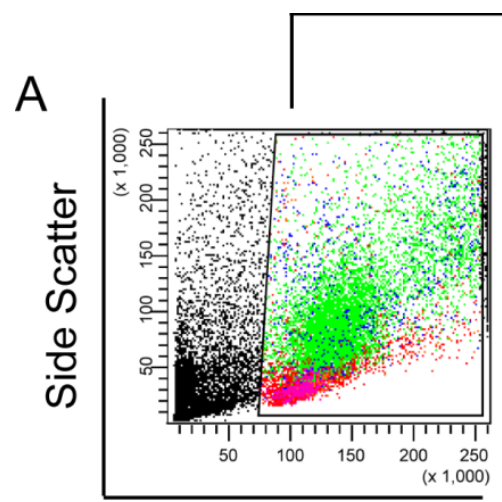

Forward Scatter

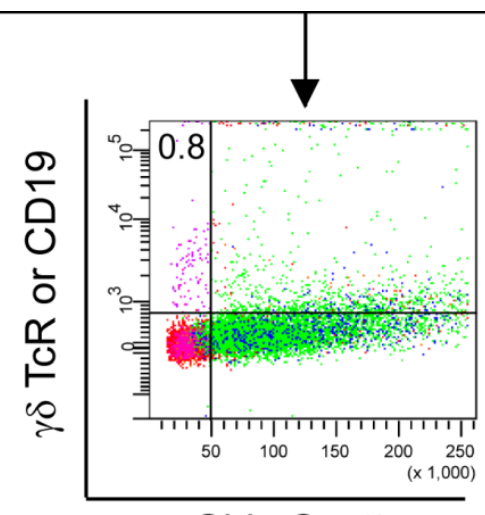

Side Scatter

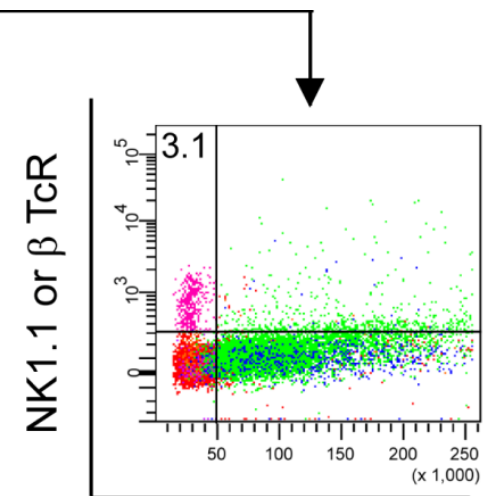

Side Scatter

B

$\beta \mathrm{TcR}+\mathrm{SSc}{ }^{\text {low }}$
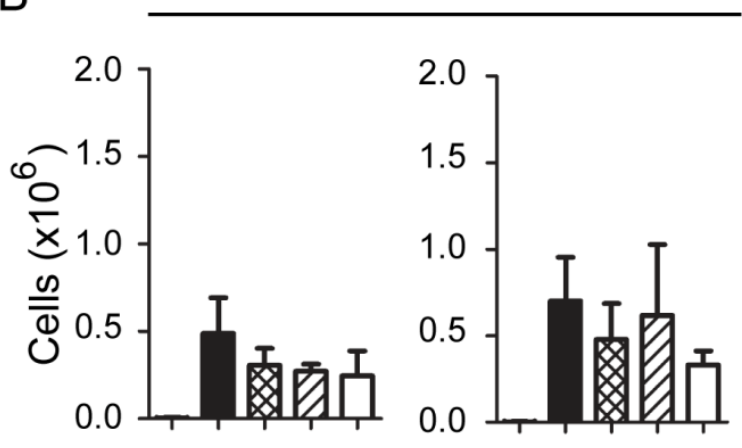

Day 20

Day 18

$\gamma \delta \mathrm{TcR}+\mathrm{SSc}^{\text {low }}$

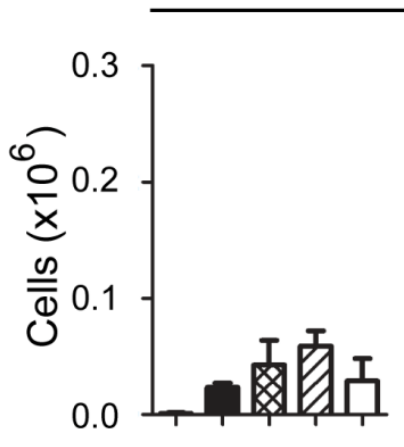

0.3

Day 18

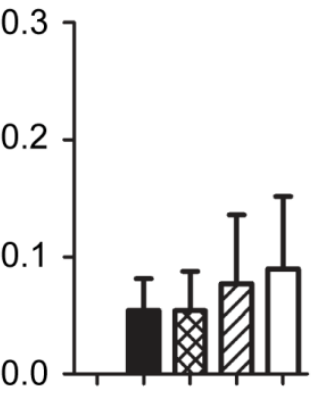

Day 20

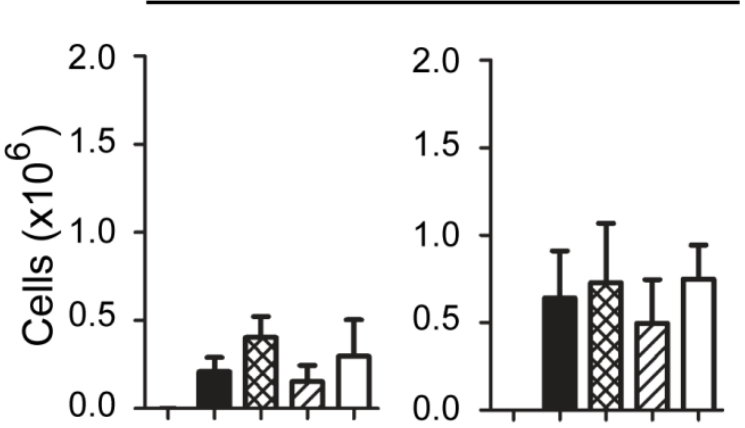

Day 18

Day 20

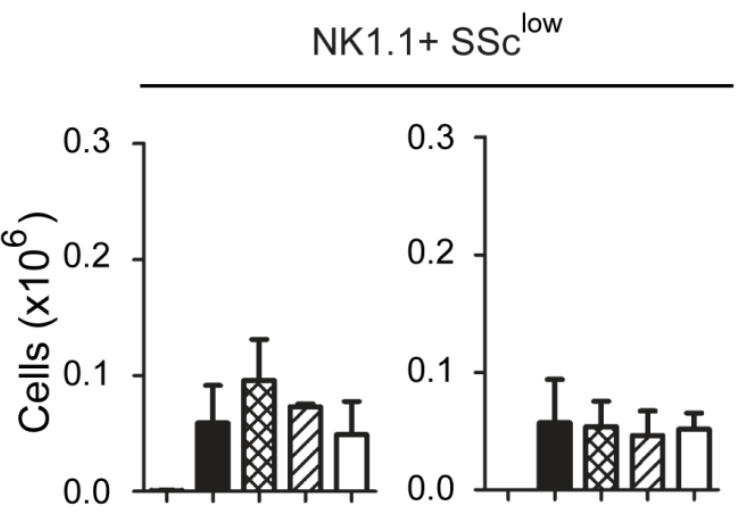

Day 18

WT Unexp

WT 3wk SR exp

区X TLR2 $^{-/-} 3 w k$ SR exp

ZZ $\mathrm{TLR}^{-/-} 3 w \mathrm{wk} \mathrm{SR}$ exp

$\square \mathrm{TLR} 2 / 9^{-/-} 3 \mathrm{wk}$ SR exp 

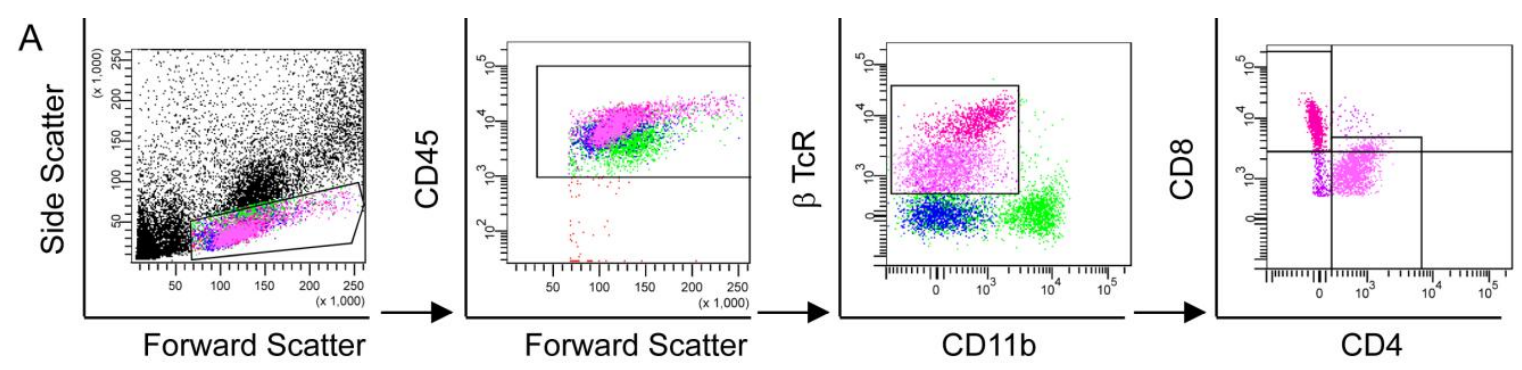

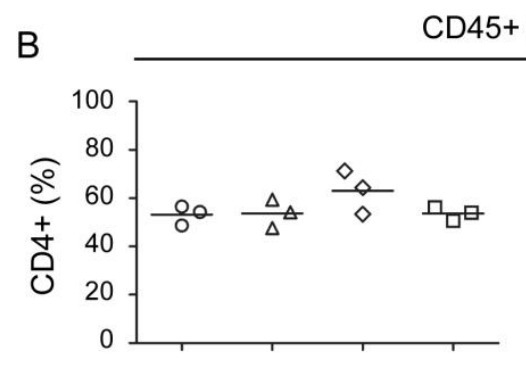

Day 18
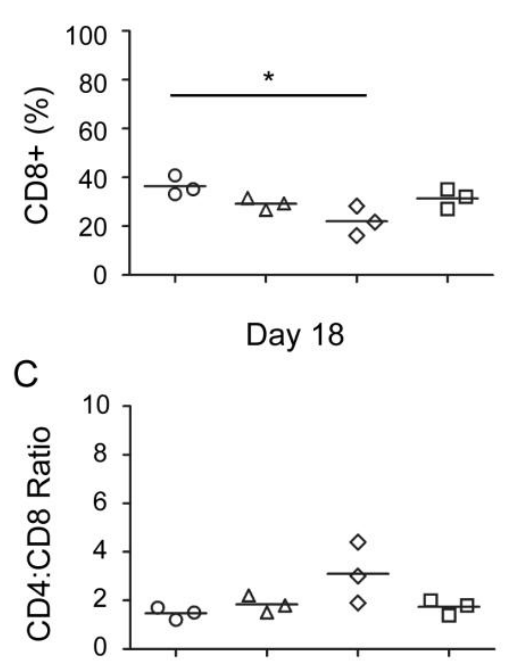

Day 18

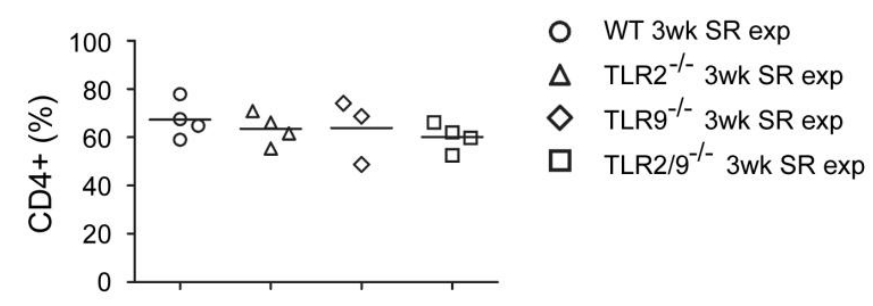

Day 20

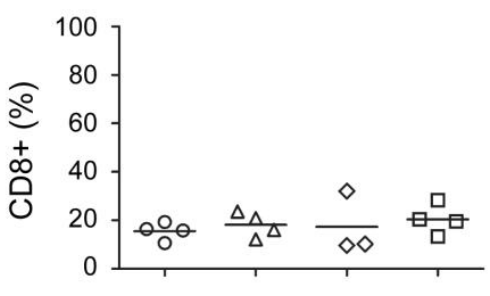

Day 20

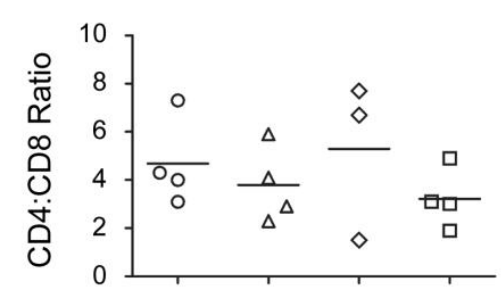

Day 20

Figure 4-4. $\mathrm{CD4}^{+}$and $\mathrm{CD8}^{+}$Th cells are recruited to the airways of mice exposed to SR for 3 weeks.

WT, TLR2 ${ }^{-/-}$, TLR $^{-/-}$, and TLR2/ $9^{-/-}$mice were exposed to SR 3 times/week for 3 weeks and analyzed on day 18 (1 day after the last exposure) or day 20 (3 days after the last exposure). BAL was performed and cells recovered from the BAL fluid were incubated with antibody to CD45, CD11b, $\beta$ TcR, CD4, and CD8 and analyzed by flow cytometry. (A) The gating strategy to discriminate $\mathrm{CD}^{+}$Th cells $\left(\mathrm{CD} 45^{+} / \mathrm{CD} 1 \mathrm{~b}^{-}\right.$/ $\left.\beta \mathrm{TcR}^{+} / \mathrm{CD}^{+}\right)$and $\mathrm{CD} 8^{+} \mathrm{T}$ cells $\left(\mathrm{CD} 45^{+} / \mathrm{CD} 1 \mathrm{~b}^{-} / \beta \mathrm{TcR}^{+} / \mathrm{CD}^{+}\right)$. (B) The percentage of $\mathrm{CD}^{+} \mathrm{Th}$ cells and $\mathrm{CD} 8^{+} \mathrm{T}$ cells present in the BAL. (C) The CD4:CD8 ratio was calculated. ( $n=3$ - 4 mice per group). The data represent mean \pm SD and significance was determined using one-way ANOVA with Tukey post-hoc test $\left(^{*} p<0.05\right.$ compared to WT SR exposed mice). 
$C D 45+\beta T c R+C D 11 b-$

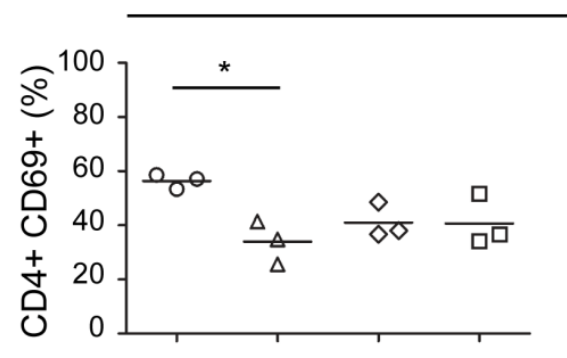

Day 18

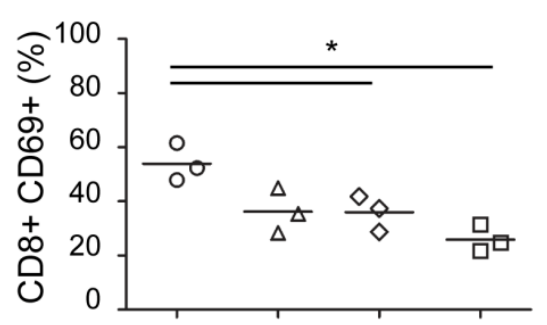

Day 18

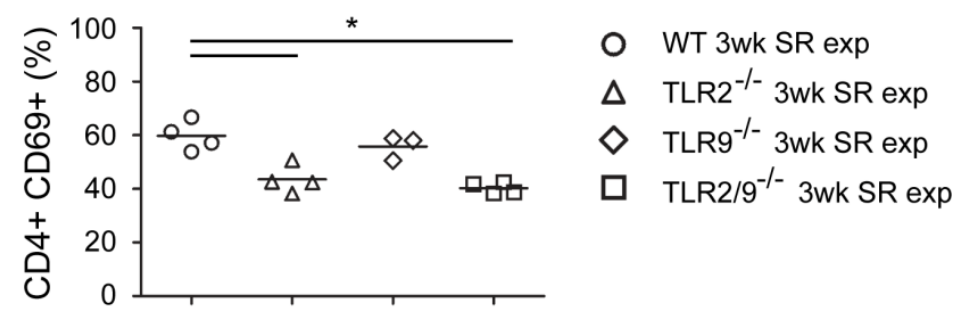

Day 20

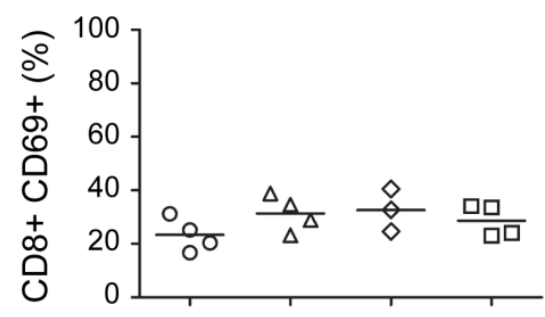

Day 20

Figure 4-5. Activated $\mathrm{CD}^{+}$and $\mathrm{CD8}^{+} \mathrm{T}$ cells in the $\mathrm{BAL}$ of mice exposed to $\mathrm{SR}$ for 3 weeks is reduced in TLR2/9 ${ }^{-/-}$mice.

WT, TLR2 ${ }^{-/-}$, TLR9 ${ }^{-/-}$, and TLR2 $/ 9^{-/-}$mice were exposed to SR 3 times/week for 3 weeks and analyzed on day 18 ( 1 day after the last exposure) or day 20 (3 days after the last exposure). BAL was performed and cells recovered from the BAL fluid were incubated with antibody to CD45, CD11b, $\beta$ TcR, CD4, CD8, and CD69 and analyzed by flow cytometry. The cells were gated to discriminate activated $\mathrm{CD} 4^{+}$Th cells $\left(\mathrm{CD} 45^{+} /\right.$

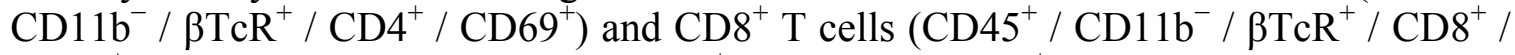
$\left.\mathrm{CD} 9^{+}\right)$. The percentage of activated $\mathrm{CD} 4^{+}$Th cells and $\mathrm{CD} 8^{+} \mathrm{T}$ cells present in the BAL. ( $n=3-4$ mice per group). The data represent mean $\pm \mathrm{SD}$ and significance was determined using one-way ANOVA with Tukey post-hoc test $(* p<0.05$ compared to WT SR exposed mice). 
important for $\mathrm{CD}^{+}$Th cell activation. In contrast, TLR9 $9^{-/}$and TLR2/9 $9^{-/}$mice had a significant reduction in the percentage of $\mathrm{CD} 69^{+} \mathrm{CD}^{+} \mathrm{T}$ cells on day 18 (Figure 4-5). This suggests signaling through TLR9 may be important for $\mathrm{CD} 8^{+} \mathrm{T}$ cell activation. The percentage of B cells and NK cells expressing CD69 was low, and there was no significant difference between WT and KO groups (Figure 4-6). Although $\gamma \delta \mathrm{T}$ cells were not a dominant cell type in the airways on day 18 and day 20, more than half express CD69 suggesting they are activated in response to SR and contribute to the immune response (Figure 4-6).

\section{Granuloma Formation}

\section{TLR2 $/ 9^{-/}$Mice Have a Significant Reduction in the Percentage of Activated $T$ and $B$ Cells in the Lung}

With repeated SR exposures an adaptive immune response develops, which is associated with $\mathrm{T}$ cell dependent granuloma formation in the lung. To investigate the role of TLRs 2 or 9 in lymphocyte populations in the lung, we exposed WT, TLR2 ${ }^{-/}$, TLR9 ${ }^{-/}$, and TLR2 $/ 9^{-/-}$mice to SR 3 times/week for 3 weeks and sacrificed the mice on day 20 ( 3 days after the last exposure) and analyzed immune cell populations within the interstitial lung tissue by flow cytometry. $\mathrm{CD}^{+}$Th cells were identified as $\mathrm{CD} 45^{+}$/ $\beta \mathrm{TcR}^{+} / \mathrm{CD}^{+}$. There was a significant reduction in the percentage of $\mathrm{CD}^{+} \mathrm{Th}$ cells in the lung in TLR2 ${ }^{-/}$and TLR $2 / 9^{-/-}$mice on day 20 compared to WT exposed mice (Figure 4-7A). The reduced percentage of Th cells in the lungs of TLR2 ${ }^{-/}$and TLR $2 / 9^{-/-}$mice was also associated with a significant reduction in the percentage of Th cells that express the activation marker CD69 (Figure 4-7B), suggesting signaling through TLR2 is important for $\mathrm{CD}^{+}{ }^{+}$Th cell recruitment and activation in response to SR.

CD25 is the high affinity IL-2 receptor, and is transiently upregulated on activated proliferating $\mathrm{T}$ cells. Although not expressed on effector $\mathrm{T}$ cells at the site of infection, CD25 expression is retained on Treg cells. There was no significant difference in the percentage of Th cells that express CD25 in the lungs of WT or KO mice exposed to SR (Figure 4-7B). Therefore, the reduction in the percentage of activated $\mathrm{CD} 4^{+} \mathrm{Th}$ cells in the lungs of TLR $2 / 9^{-/-}$mice was not associated with an increased percentage of CD25 $\mathrm{CD}^{+}$Treg cells.

Upon antigen stimulation, effector $\mathrm{CD} 4^{+}$Th cells and other lymphocytes must be recruited to sites of infection. Like neutrophils, and monocytes, lymphocytes express chemokine receptors to traffic to site of infection. Previous studies have shown that CXCL9, CXCL10, and CXCL11 are induced by SR exposure, and recruit T cells that express CXCR3 (Nance et al., 2004). The expression of CCR6, which binds to CCL20, has been associated with the recruitment of Th17 cells as well as Treg cells (Yamazaki et al., 2008). The $\mathrm{CD}^{+}{ }^{+}$Th cells recruited to the lung of SR exposed mice expressed the 
CD45+ CD19+ CD11b-

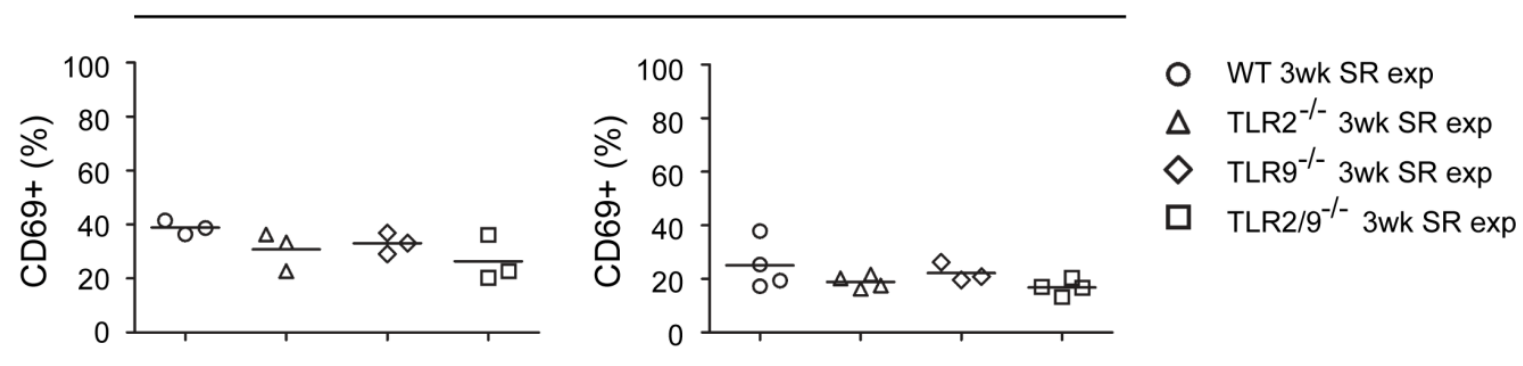

Day 18

Day 20

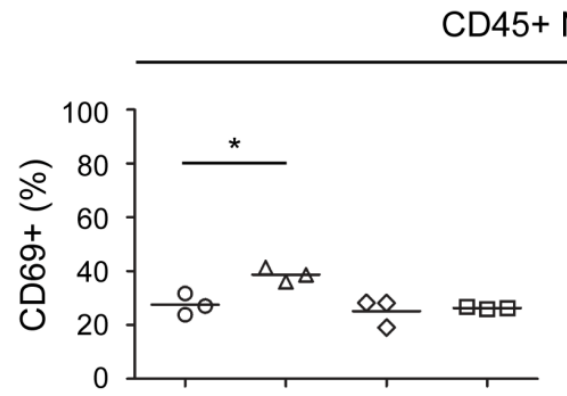

Day 18

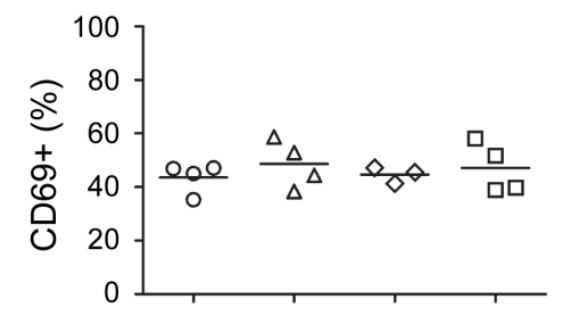

Day 20

$\mathrm{CD} 45+\gamma \delta \mathrm{TcR}+\mathrm{CD} 11 \mathrm{~b}-$

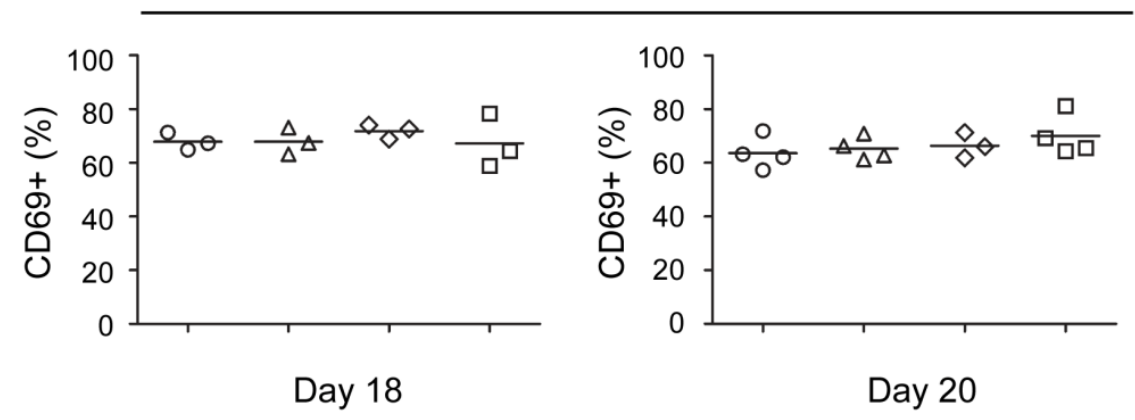

Figure 4-6. TLRs 2 and 9 are not required for activation of B cells, NK cells, or $\gamma \delta$ $T$ cells in the airways of mice exposed to SR for 3 weeks.

WT, TLR2 ${ }^{-/-}$TLR9 $^{-/-}$, and TLR2/9 ${ }^{-/-}$mice were exposed to SR 3 times/week for 3 weeks and analyzed on day 18 ( 1 day after the last exposure) or day 20 (3 days after the last exposure). BAL was performed and cells recovered from the BAL fluid were incubated with antibody to CD45, CD11b, CD19, NK1.1, $\gamma \delta \mathrm{TcR}$, and CD69 and analyzed by flow cytometry. The cells were gated to discriminate activated $\mathrm{B}$ cells $\left(\mathrm{CD} 45^{+} / \mathrm{CD} 11 \mathrm{~b}^{-} / \mathrm{CD} 9^{+} / \mathrm{CD}^{+} 9^{+}\right)$, NK cells $\left(\mathrm{CD} 45^{+} / \mathrm{CD} 1 \mathrm{~b}^{-} / \mathrm{NK} 1.1^{+} / \mathrm{CD}^{+} 9^{+}\right)$, and

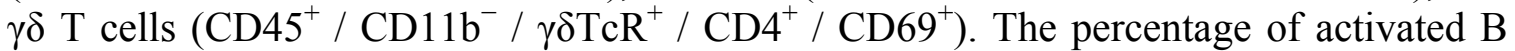
cells, NK cells, and $\gamma \delta \mathrm{T}$ cells present in the BAL ( $n=3-4$ mice per group). The data represent mean $\pm \mathrm{SD}$ and significance was determined using one-way ANOVA with Tukey post-hoc test $(* p<0.05$ compared to WT SR exposed mice). 
Figure 4-7. Reduced activation of $\mathrm{CD}^{+}$Th cells in the lungs of $\mathrm{TLR2}^{-/-}$and TLR2 $/ 9^{-/-}$mice exposed to SR for 3 weeks.

WT, TLR2 ${ }^{-/}$, TLR $^{-/-}$, and TLR2/ $9^{-/-}$mice were exposed to SR 3 times/week for 3 weeks and analyzed on day 20 ( 3 days after the last exposure). Lung cells were digested and incubated with antibodies to CD45, $\beta$ TcR, CD4, CD69, CD25, and the chemokine receptors CXCR3, and CCR6 and analyzed by flow cytometry. (A) The percentage of $\mathrm{CD}^{+}$Th cells $\left(\mathrm{CD}^{+} 5^{+} / \mathrm{CD}^{+} / \beta \mathrm{TcR}^{+}\right)$in the lymphocyte population were identified. (B) The percentage of $\mathrm{CD}^{+}$Th cells that express CD69, CD25, CXCR3, or CCR6 were analyzed. ( $n=3-4$ mice per group. The lung cells from 3 or 4 unexposed mice were combined). The data represent mean $\pm \mathrm{SD}$ and significance was determined using oneway ANOVA with Tukey post-hoc test $\left({ }^{*} p<0.05\right.$ compared to WT SR exposed mice). 


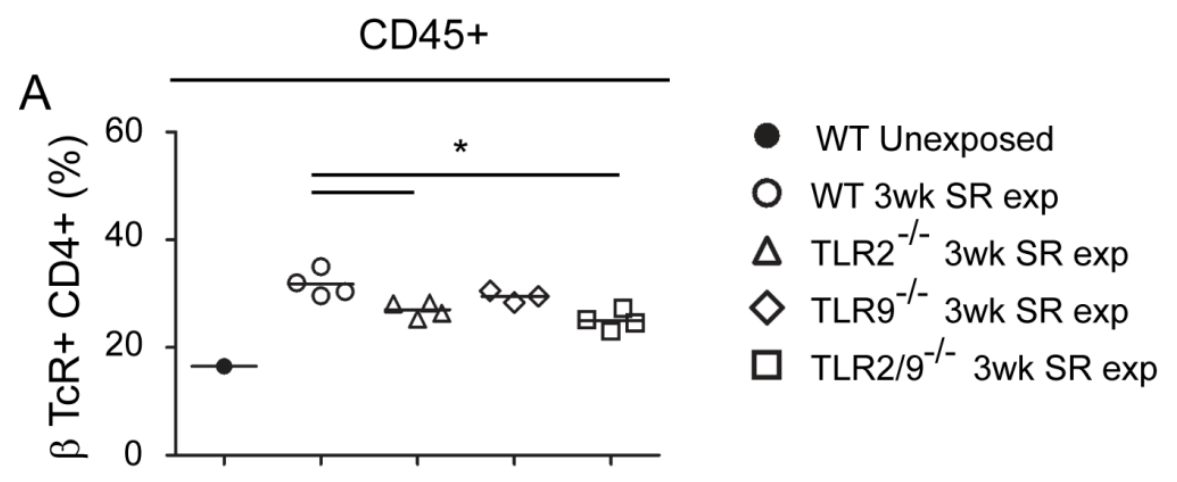

Day 20

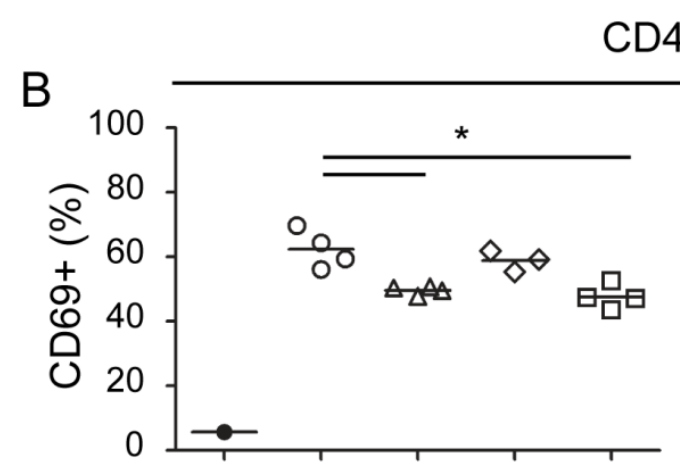

Day 20

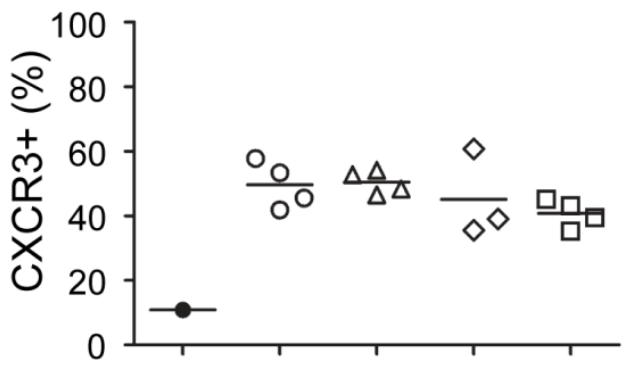

Day 20

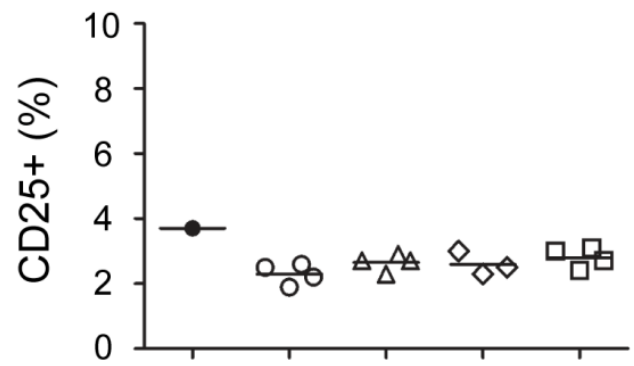

Day 20

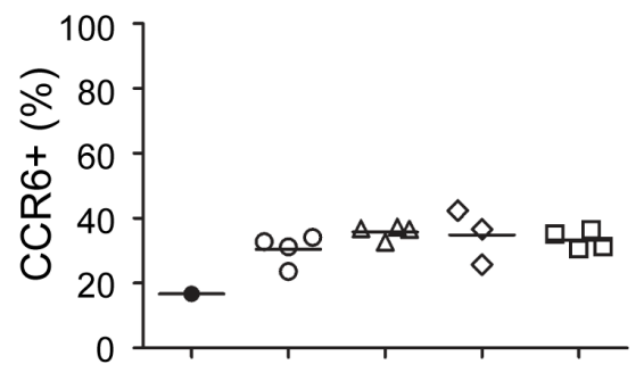

Day 20 
chemokine receptors $\mathrm{CXCR} 3$ and CCR6, but there was no difference in the percentage among WT and KO mice (Figure 4-7B).

\section{TLR2 Is the Dominant Receptor for Cytokine Production Following Repeated SR Exposures}

The reduced cellular recruitment to the lungs and reduced activation of lymphocytes could be due to a reduced inflammatory environment in the lungs of TLR2 $/ 9^{-/-}$mice. Studies during the acute phase showed TLRs 2 and 9 were involved in the expression of TNF $\alpha$, IL-6, IL-12p40, and IL-23p19. To determine if inflammation is reduced in TLR2 $/ 9^{-/-}$mice following repeated SR exposures, the expression of TNF $\alpha$, IL-6, IL-10, IL-12p40, and IL-23p19 in the lung tissue of mice exposed to SR for three weeks was measured by qRT-PCR (Figure 4-8). TNF $\alpha$ mRNA was significantly decreased in the lungs of TLR2 ${ }^{--}$and TLR $2 / 9^{--}$mice compared to the WT mice following 3 weeks of exposure. The expression of IL-6 and IL-23p19 was increased over unexposed mice, but not significantly reduced in KO mice compared to WT SR exposed mice. We did not detect an increase of IL-10 mRNA in the lungs of TLR2/9 ${ }^{-/-}$mice exposed for 3 weeks, indicating that a decrease in $\mathrm{T}$ and $\mathrm{B}$ cell activation is not related to expression of IL-10.

The Th17 response is associated with increased disease severity and fibrosis in HP (Joshi et al., 2009; Simonian et al., 2009). Th17 cells are polarized in response to IL-6 and TGF $\beta$ and proliferate in response to IL-23, and the expression of IL-6 and IL-23p19 was increased in SR exposed lungs. IFN $\gamma$, a Th1 associated cytokine, is also critical for granuloma formation in HP. To investigate to role of TLRs 2 and 9 in the expression of Th17 associated cytokines IL-17 and IL-22 and Th1 associated cytokine IFN $\gamma$ after 3 weeks exposure to SR, the expression of IL-17, IL-22, and IFN $\gamma$ in the lung tissue was measured by qRT-PCR (Figure 4-9). Expression of IL-17 and IL-22 was significantly reduced in the TLR2 $2^{-/-}$and TLR2 $/ 9^{-/-}$mice compared to the WT. Expression of IFN $\gamma$ was significantly reduced in TLR9 ${ }^{-/}$mice in comparison to WT mice following SR exposure suggesting that TLR9 is important in IFN $\gamma$ expression. Altogether, these results suggest that TNF $\alpha$, IL-17, and IL-22 production is largely mediated by TLR 2 stimulation following repeated exposure with SR.

\section{TLR2 $/ 9^{-/-}$Mice Have a Reduced Percentage of Th17 Cells in the Lung}

Because TLR2 $/ 9^{-/-}$mice had a reduced percentage of activated CD4 $4^{+}$Th cells and Th17 associated cytokine expression, we next wanted to know if TLR $2 / 9^{-/-}$mice were protected from developing a Th17 response. Mice were exposed to SR 3 times a week for 3 weeks and sacrificed on day 18 ( 1 day after the last exposure) and the percentage of Th17 and Th1 cells in the lungs was measured in ex vivo SR lysate stimulated lung cells by intracellular flow cytometry. The gating strategy for the detection of $\mathrm{CD}^{+} \mathrm{Th}$ cells is

depicted in Figure 4-10A. The results show the percentage of $\mathrm{CD}^{+}$Th cells that 

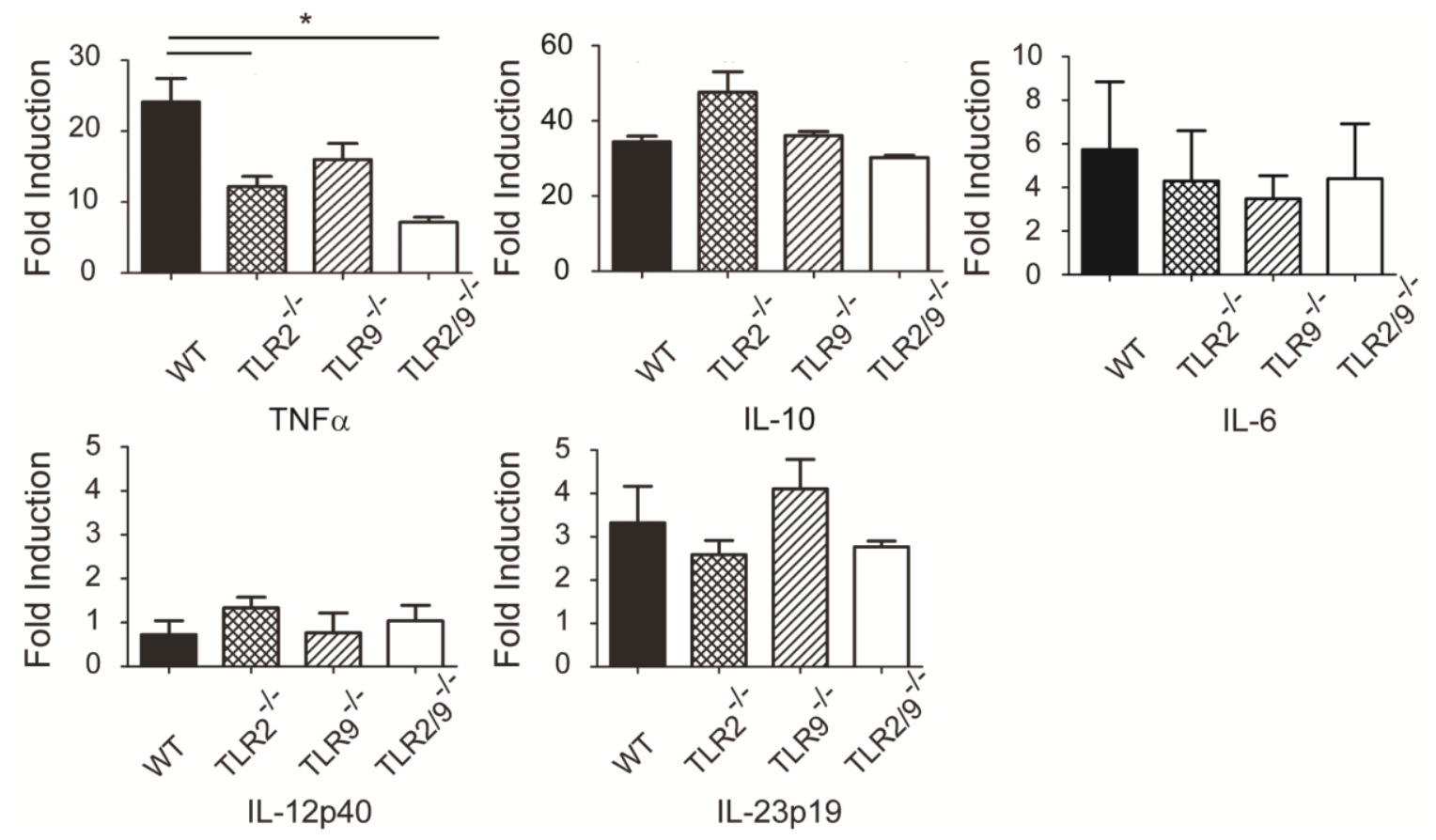

Figure 4-8. TLRs 2 and 9 contribute to TNFa expression following repeated SR exposures.

$\mathrm{WT}, \mathrm{TLR}^{-/}{ }^{-}, \mathrm{TLR}^{-/-}$, and TLR2 $/ 9^{-/-}$mice were exposed to SR 3 times/week for 3 weeks and analyzed on day 18 (1 day after the last exposure). RNA was isolated from lung tissue and reverse transcribed to cDNA for real time qRT-PCR to measure the expression of TNF $\alpha$, IL-10, IL-6, IL-12p40, and IL-23p19 using specific primers. The results were normalized to the housekeeping gene HPRT and expressed as mean $\pm \mathrm{SD}$ fold induction over unexposed mice. ( $\mathrm{n}=3$ mice / group) Significance was determined using one-way ANOVA with Tukey post-hoc test $(* \mathrm{p}<0.05$ compared to WT SR exposed mice). 

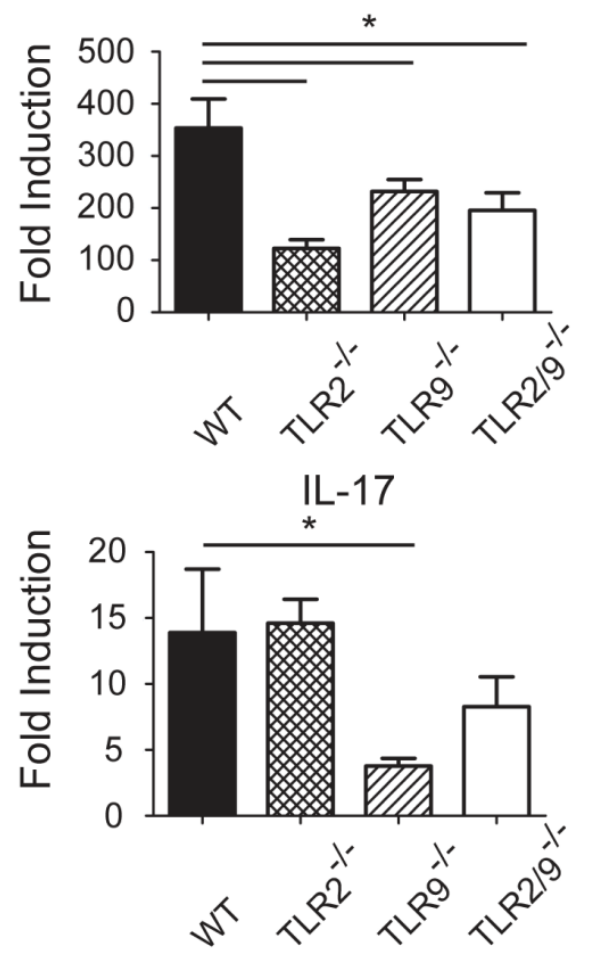

IFN $\gamma$

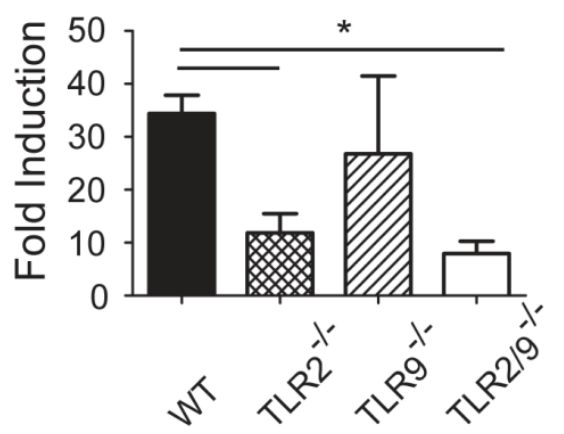

IL-22

Figure 4-9. TLRs 2 and 9 contribute to Th1- and Th17-associated cytokine production following repeated SR exposures.

WT, TLR2 ${ }^{-/-}$, TLR9 ${ }^{-/-}$, and TLR2/9 ${ }^{-/-}$mice were exposed to SR 3 times/week for 3 weeks and analyzed on day 18 ( 1 day after the last exposure). RNA was isolated from lung tissue and reverse transcribed to cDNA for real time qRT-PCR to measure the expression of IFN $\gamma$, IL-17, and IL-22 using specific primers. The results were normalized to the housekeeping gene HPRT and expressed as mean \pm SD fold induction over unexposed mice. ( $\mathrm{n}=3$ mice / group) Significance was determined using one-way ANOVA with Tukey post-hoc test ( ${ }^{*} p<0.05$ compared to WT SR exposed mice). 
Figure 4-10. TLRs 2 and 9 are necessary for the generation of the Th17 response following 3 weeks repeated SR exposure.

WT, TLR2 ${ }^{-/-}$, $\mathrm{TLR}^{-/-}$, and TLR2 $/ 9^{-/-}$mice were exposed to SR 3 times/week for 3 weeks and analyzed on day 18 ( 1 day after the last exposure). Lung cells were digested and stimulated with SR lysate or media alone in the presence of brefeldin $A$ then incubated with antibodies for CD45, $\beta$ TcR, CD4, IL-17A, and IFN $\gamma$ for intracellular cytokine staining. (A) The gating strategy to discriminate $\mathrm{CD} 4^{+}$Th cells $\left(\mathrm{CD} 45^{+} / \mathrm{CD} 4^{+} /\right.$ $\beta \mathrm{TcR}^{+}$). (B) Representative dot plots of IL-17A and IFN $\gamma$ expressing CD4 ${ }^{+}$Th cells. (C) The percentage of $\mathrm{CD}^{+}$Th cells expressing IL-17A and/or IFN $\gamma$. ( $\mathrm{n}=3$ mice / group. The lung cells from 3 unexposed mice were combined.) Significance was determined using two-way ANOVA with Bonferroni post-hoc test $\left({ }^{*} p<0.05\right.$ compared to WT SR exposed mice). 

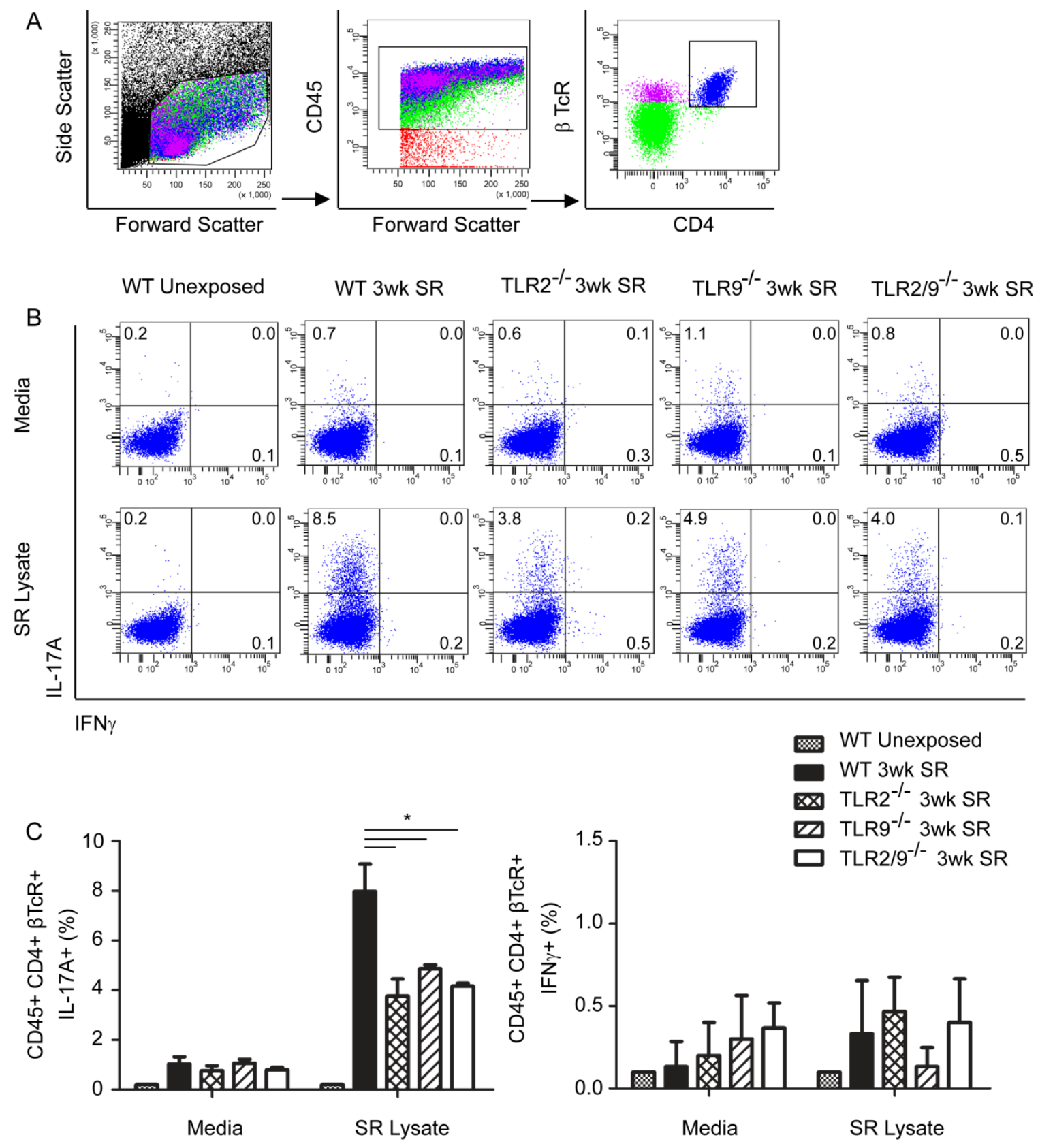
expressed IL-17A and IFN $\gamma$ (Figure 4-10B). In WT mice, $8 \%$ of $\mathrm{CD}^{+}$Th cell stimulated with SR lysate respond with IL-17A production (Figure 4-10C). TLR2 ${ }^{-/-}$, TLR9 ${ }^{-/}$, and

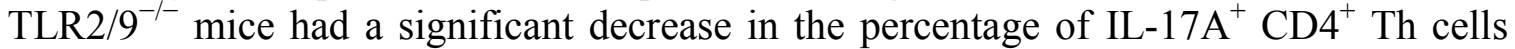
compared to WT exposed mice, indicating TLRs 2 and 9 both contribute to the Th17 response. Less than $1 \%$ of Th cells stimulated with SR lysate responded with IFN $\gamma$ production, suggesting Th17 cells play a greater role in the Th cell response at this time point.

To determine if the Th17 response is maintained 4 days after the last exposure, the percentage of Th17 cells in the lung was measured on day 21 . In addition, $\mathrm{CD} 4^{+} \mathrm{Th}$ cells were stained for the expression of CD25, which is not expressed on effector Th17 cells. The results show the percentage of $\mathrm{CD}^{+}$Th cells that expressed CD25 / IL-17A (Figure 4-11A) and CD25 / IL-17F, an alternative IL-17 isoform secreted by Th17 cells (Figure 4-11B). IL-17A expressing $\mathrm{CD} 25^{-} \mathrm{CD} 4^{+}$Th cells were still detected in WT, $\mathrm{TLR}^{-/}{ }^{-1} \mathrm{TLR}^{-/-}$mice, but the percentage of Th17 cells in TLR2 $/ 9^{-/-}$mice was significantly reduced compared to WT exposed mice (Figure 4-11C). IL-17F expressing $\mathrm{CD}^{+}$Th cells were not detected at this time point (Figure 4-11C). Altogether, these results suggest that signaling through TLRs 2 and 9 are necessary for sustaining a Th17 response in the lung after 3 weeks exposure to SR.

The reduction in Th17 cells on day 21 in TLR $2 / 9^{-/-}$mice could be due to an increase in the percentage of IFN $\gamma$ or TNF producing Th cells. Plate bound anti-CD3 antibody, a polyclonal stimulator of $\mathrm{T}$ cells, was used on day 21 in case the low percentage of IFN $\gamma$ producing $\mathrm{CD}^{+}$Th cells observed on day 18 was due to poor stimulation of IFN $\gamma$ by SR lysate. Mice were exposed to SR 3 times a week for 3 weeks and sacrificed on day 21 (4 days after the last exposure) and the percentage of IFN $\gamma^{+}$and $\mathrm{TNF}^{+}$cells in stimulated dispersed lung cells was detected by intracellular flow cytometry. The results show the percentage of $\mathrm{CD}^{+}$Th cells expressing CD25 / IFN $\gamma$ (Figure 4-12A) and CD25 / TNF $\alpha$ (Figure 4-12B). An average of $2.8 \%$ of CD25 $5^{-} \mathrm{CD} 4^{+}$ Th cells expressed IFN $\gamma$ and $7.5 \%$ expressed TNF $\alpha$. The percentage IFN $\gamma^{+}$Th1 cells was significantly reduced in $\mathrm{TLR} 2^{---}$mice, and the percentage of $\mathrm{TNF} \alpha^{+} \mathrm{CD} 4^{+} \mathrm{Th}$ cells was significantly reduced in TLR $2 / 9^{--}$mice compared to WT SR exposed mice (Figure 4-12C). The results suggest TLR2 may be important for Th cell expression of IFN $\gamma$, and TLRs 2 and 9 are important for Th cell expression of TNF $\alpha$. Importantly, it also shows the reduction in the Th17 response in TLR $2 / 9^{-/-}$mice is not due to an increase in IFN $\gamma$ or TNF $\alpha$ producing $\mathrm{CD}^{+}$Th cells.

We next wanted to know if the reduced percentage of Th17 cells in the lungs of TLR2 $/ 9^{-/-}$mice was due to an increase in Treg cells, identified by the expression of CD25 and the transcription factor FoxP3. Mice were exposed to SR 3 times a week for 3 weeks and sacrificed on day 21 (4 day after the last exposure) and the percentage of $\mathrm{CD} 25^{+}$and $\mathrm{FoxP}^{+}$cells in SR lysate stimulated dispersed lung cells was detected by intracellular flow cytometry. The results show the percentage of $\mathrm{CD}^{+}$Th cells expressing CD25 and FoxP3 (Figure 4-13A). No more than half a percent of CD4 ${ }^{+} \mathrm{Th}$ cells were $\mathrm{CD} 25^{+}$FoxP $^{+}$in SR exposed mice indicating few Treg cells are recruited to 
Figure 4-11. TLRs 2 and 9 cooperate in sustaining the Th17 response following 3 weeks repeated SR exposure.

WT, TLR2 ${ }^{-/-}$, $\mathrm{TLR}^{-/-}$, and TLR2 $/ 9^{-/-}$mice were exposed to SR 3 times/week for 3 weeks and analyzed on day 21 (4 days after the last exposure). Lung cells were digested and stimulated with SR lysate or media alone in the presence of monensin then incubated with antibodies for CD45, $\beta T c R, C D 4, C D 25$, IL-17A, and IL-17F for intracellular cytokine staining. (A) Representative dot plots for $\mathrm{CD}^{+}$Th cells expressing CD25 or IL-17A. (B) Representative dot plots for $\mathrm{CD}^{+}$Th cells expressing CD25 or IL-17F. (C) The percentage of $\mathrm{CD} 25^{-} \mathrm{CD}^{+}$Th cells expressing IL-17A or IL-17F. ( $\mathrm{n}=3$ mice / group. The lung cells from 3 unexposed mice were combined.) Significance was determined using two-way ANOVA with Bonferroni post-hoc test $(* p<0.05$ compared to WT SR exposed mice). 

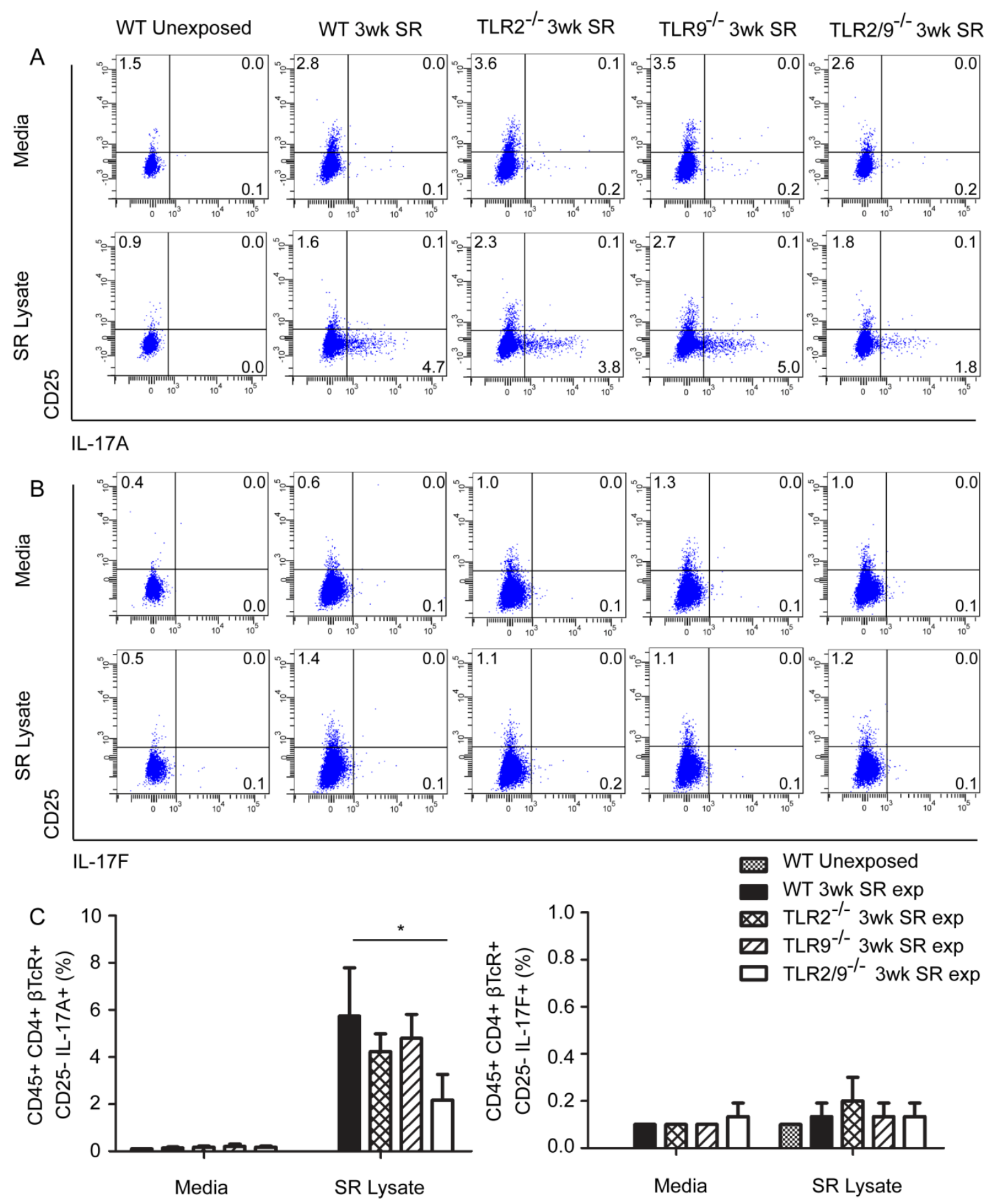
Figure 4-12. The reduction in Th17 cells in $\mathrm{TLR}_{2} / 9^{-/-}$mice is not due to an increase in $\mathrm{IFN} \gamma^{+}$or $\mathrm{TNFa}{ }^{+}$Th1 cells.

WT, TLR2 ${ }^{-/-}$, TLR9 ${ }^{-/-}$, and TLR2 $/ 9^{-/-}$mice were exposed to SR 3 times/week for 3 weeks and analyzed on day 21 (4 days after the last exposure). Lung cells were digested and stimulated with plate bound $\alpha-C D 3$ (A), SR lysate (B), or media alone in the presence of brefeldin $A$ then incubated with antibodies for CD45, $\beta T c R, C D 4, C D 25$, IFN $\gamma$, and TNF $\alpha$ for intracellular cytokine staining. (A) Representative dot plots for $\mathrm{CD}^{+}$Th cells expressing CD25 or IFN $\gamma$. (B) Representative dot plots for $\mathrm{CD}^{+}{ }^{+} \mathrm{Th}$ cells expressing CD25 or TNF $\alpha$. (C) The percentage of $\mathrm{CD} 25^{-} \mathrm{CD} 4^{+}$Th cells expressing IFN $\gamma$ or TNF $\alpha$. ( $\mathrm{n}=3$ mice / group. The lung cells from 3 unexposed mice were combined.) Significance was determined using two-way ANOVA with Bonferroni post-hoc test $(* p<$ 0.05 compared to WT SR exposed mice). 


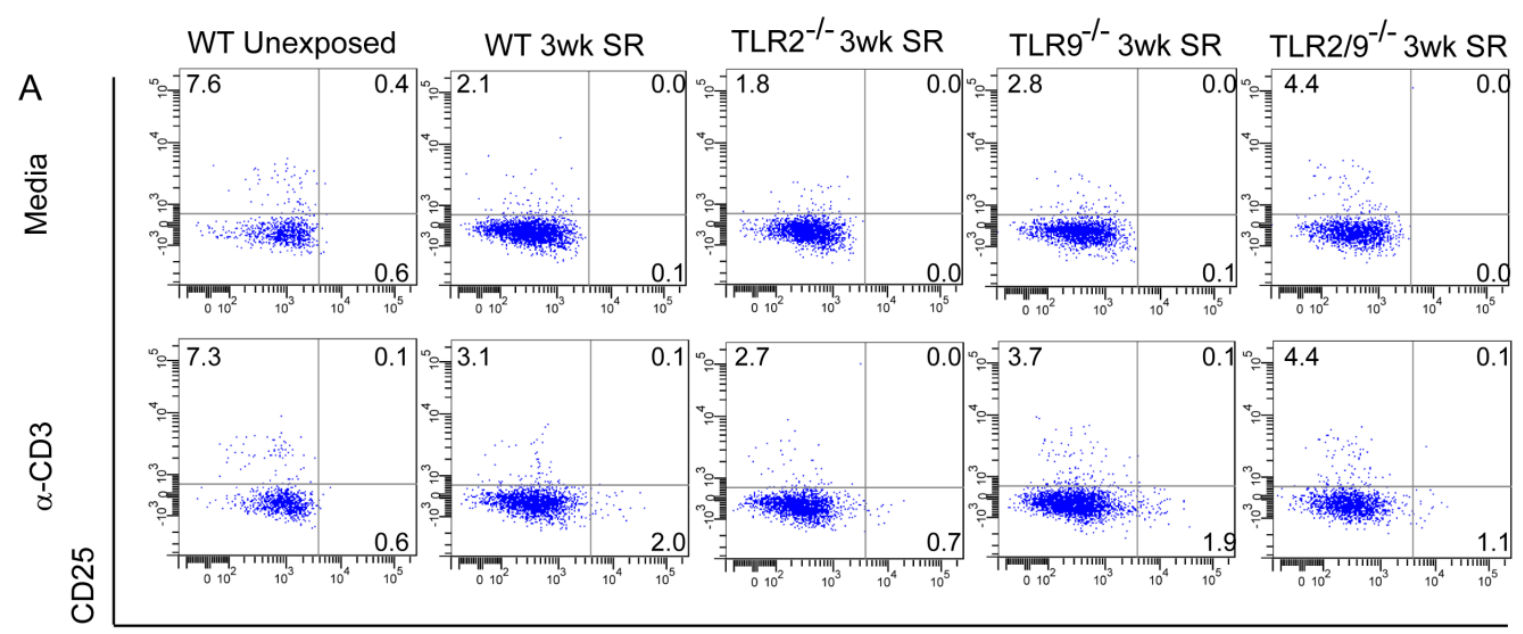

IFN $\gamma$

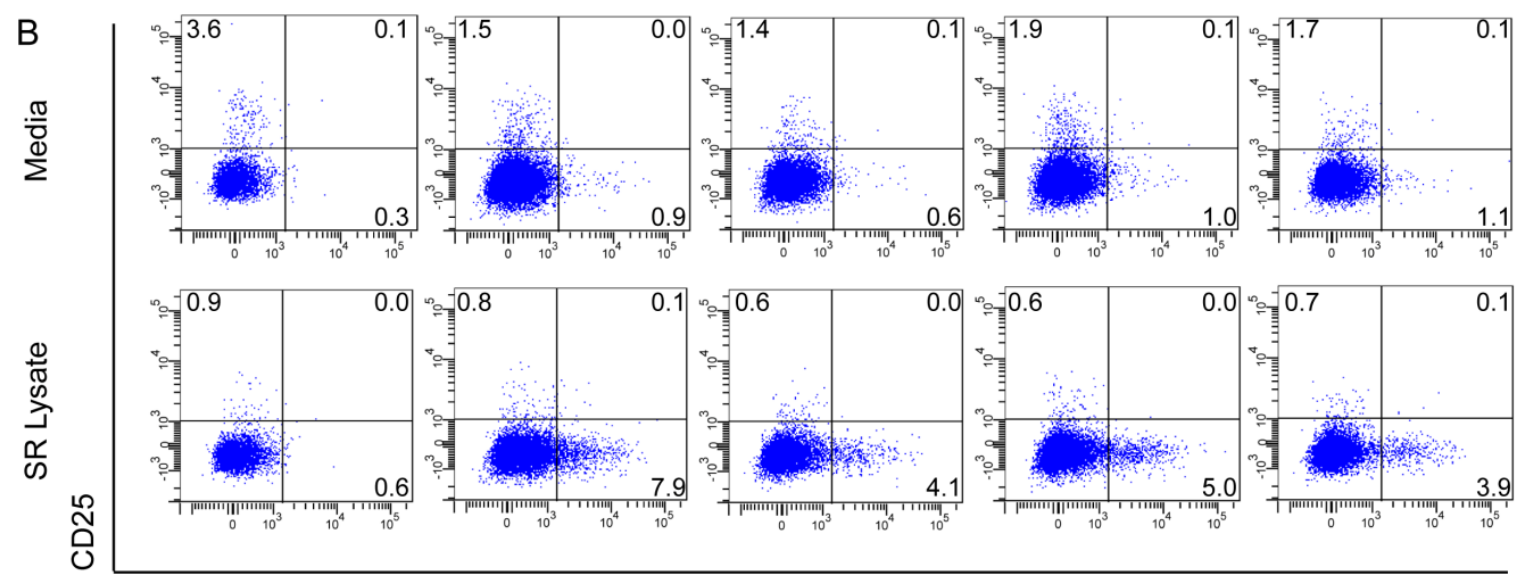

$\mathrm{TNF} \alpha$
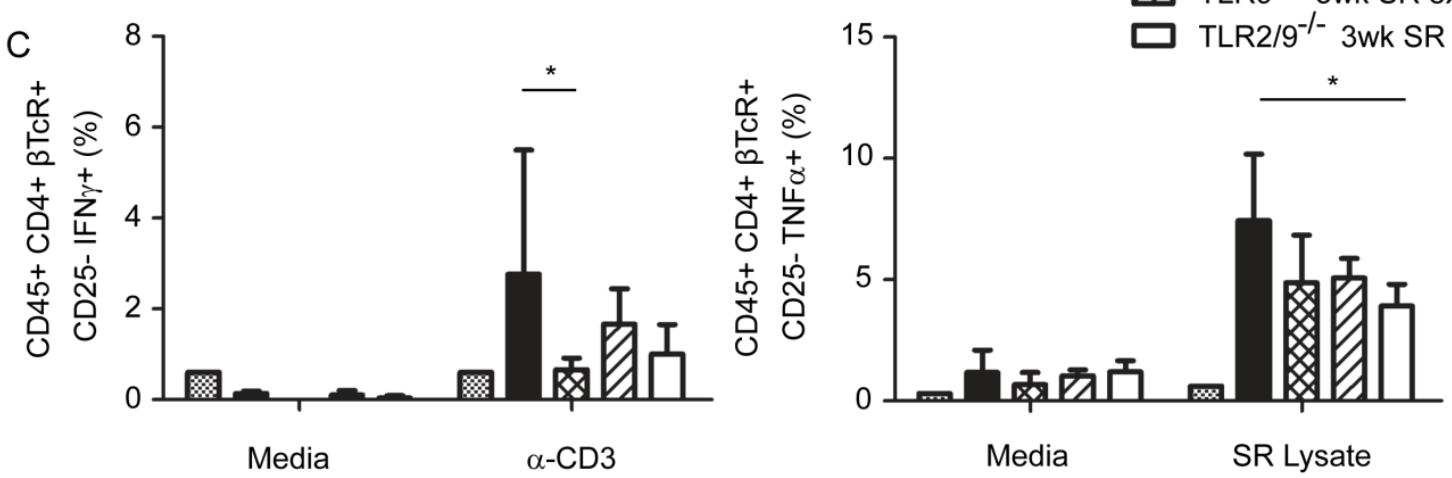


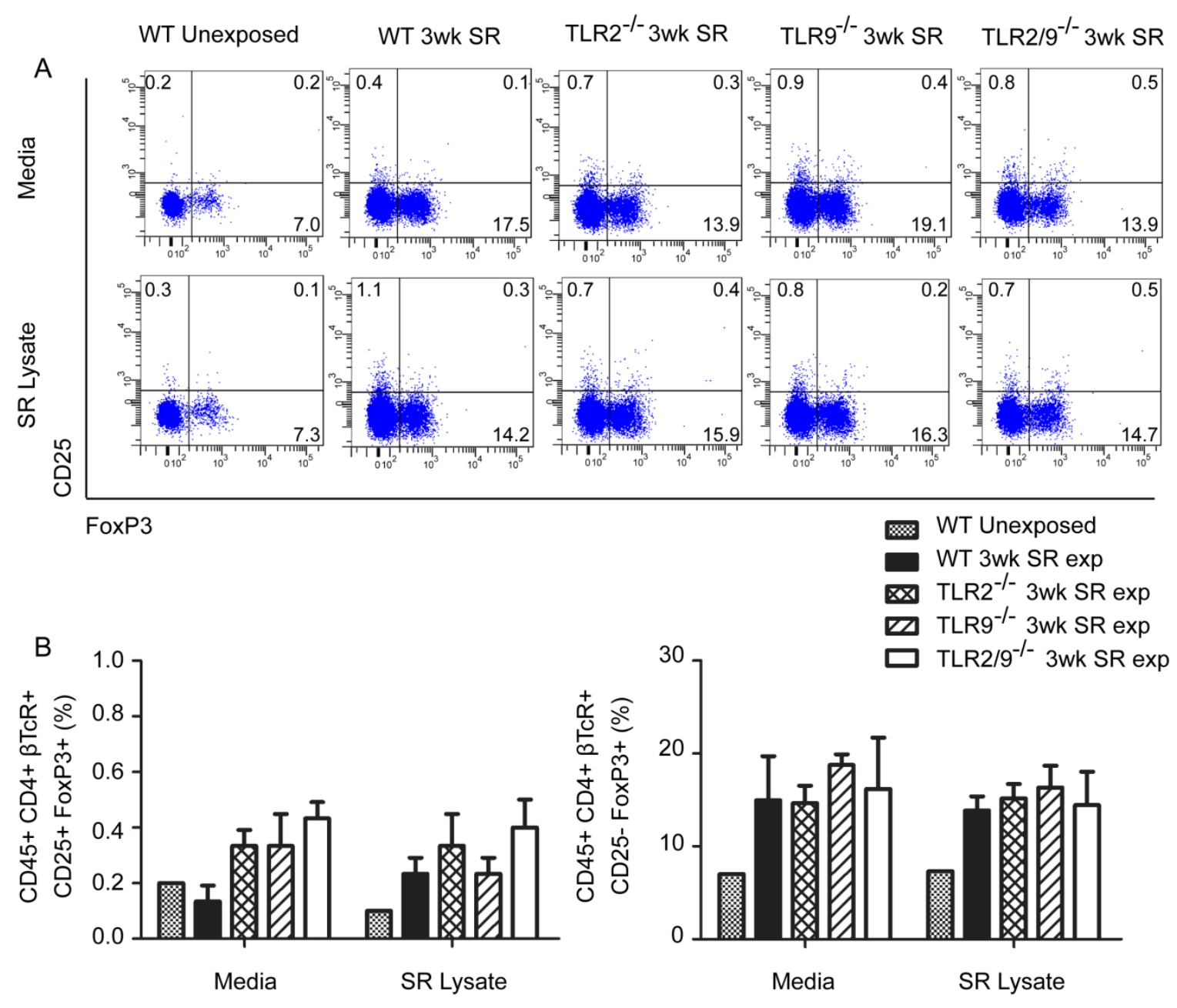

Figure 4-13. The reduction in Th17 cells in $\mathrm{TLR}_{2} / 9^{-/}$mice is not due to an increase in $\mathrm{FoxP3}^{+} \mathrm{T}$ regulatory cells.

WT, TLR2 ${ }^{-/-}$, $\mathrm{TLR}^{-/-}$, and TLR2 $/ 9^{-/-}$mice were exposed to SR 3 times/week for 3 weeks and analyzed on day 21 (4 days after the last exposure). Lung cells were digested and stimulated with SR lysate or media alone in the presence of monensin then incubated with antibodies for CD45, $\beta$ TcR, CD4, CD25, FoxP3 for intracellular cytokine staining. (A) Representative dot plots for $\mathrm{CD}^{+} \mathrm{Th}$ cells expressing CD25 or FoxP3. (B) The percentage of $\mathrm{CD} 25^{+} \mathrm{CD} 4^{+}$Th cells or $\mathrm{CD} 25^{-} \mathrm{CD} 4^{+}$Th cells expressing FoxP3. ( $\mathrm{n}=3$ mice / group. The lung cells from 3 unexposed mice were combined.) Significance was determined using two-way ANOVA with Bonferroni post-hoc test $(* p<0.05$ compared to WT SR exposed mice). 
the lung after SR exposure at this time point (Figure 4-13B). However, exposure to TGF $\beta$ at the site of infection can induce CD25 ${ }^{-} \mathrm{CD} 4^{+}$Th cells to become a Treg cells and upregulate FoxP3 (Fantini et al., 2004; Fu et al., 2004), and 14\% of the CD25 CD4 ${ }^{+}$Th cells express FoxP3 in WT SR exposed mice. There was no significant difference in the percentage of CD25 $5^{-} \mathrm{CD} 4{ }^{+}$Th cells that express FoxP3 in KO mice compared to WT SR exposed mice. Altogether, these results suggest the reduced Th17 response in TLR2/9 ${ }^{-/-}$ mice is not due to increased polarization of Treg cells.

The production of IL-10 by Treg cells can inhibit immune responses. Although the percentage of Treg cells was not different between WT and KO mice, we next wanted to determine if the reduced percentage of Th17 cells in the TLR2 $/ 9^{-/-}$mice was due to increased production of IL-10 by Treg cells. Mice were exposed to SR 3 times a week for 3 weeks and sacrificed on day 21 (4 day after the last exposure) and the percentage of $\mathrm{CD} 25^{+}$and IL-10 ${ }^{+}$Th cells in SR lysate stimulated lung cells was detected by intracellular flow cytometry. The results show the percentage of CD4 ${ }^{+}$Th cells expressing CD25 and IL-10 (Figure 4-14A). In WT SR exposed mice, only $1.3 \%$ of Th cells expressed IL-10. The IL-10 expressing CD4 ${ }^{+}$Th cells were CD25 $5^{-}$indicating the source of the IL-10 was not CD25 $5^{+}$Treg cells (Figure 4-14B). In addition, TLR2 ${ }^{-/-}$and TLR $2 / 9^{-/-}$had a significantly reduced percentage of $\mathrm{IL}-10^{+} \mathrm{CD} 25^{-} \mathrm{CD} 4^{+} \mathrm{Th}$ cells, suggesting loss of TLR2 leads to decreased IL-10 production by CD4 ${ }^{+}$Th cells, and the reduced Th17 response in TLR2/9 $9^{-/-}$mice is not due to increased IL-10-producing Th cells.

\section{Signaling Through TLR2 Is Important in B Cell Activation}

The significant reduction in CD4 ${ }^{+}$Th cells in the lungs of TLR2 ${ }^{-/-}$and TLR2/9 $9^{-/-}$ mice on day 20 and 21 was associated with a significant increase in the percentage of $B$ cells (Figure 4-15A). However, the expression of the activation marker CD69 was significantly reduced on the B cells of TLR2 ${ }^{-1-}$ and TLR $2 / 9^{-1-}$ mice on day 20 compared to WT SR exposed mice (Figure 4-15A), suggesting signaling through TLR2 is important for B cell activation. There was no significant difference in the percentage of $\mathrm{CD} 8^{+}$cells or NK cells in the lungs, nor was the percentage of $\mathrm{CD}^{+}$cells or NK cells that express of the activation maker CD69 significantly different compared to WT SR exposed mice (Figure 4-15B and $\mathbf{C}$ ). Altogether the results suggest SR induced signaling through TLR2 is important for the activation of $\mathrm{CD}^{+}{ }^{+}$Th cells and $\mathrm{B}$ cells.

\section{TLRs 2 and 9 Cooperate in Costimulatory Molecule Upregulation on Interstitial Macrophages}

Antigen specific effector $\mathrm{CD}^{+}{ }^{\mathrm{T}}$ cells are stimulated at the site of infection by antigen presenting cells, like macrophages, through the presentation of antigen on MHCII molecules. Stimulation of macrophages through pattern recognition receptors like TLR2 and TLR9 leads to the upregulation of costimulatory molecules CD80, CD86, and 

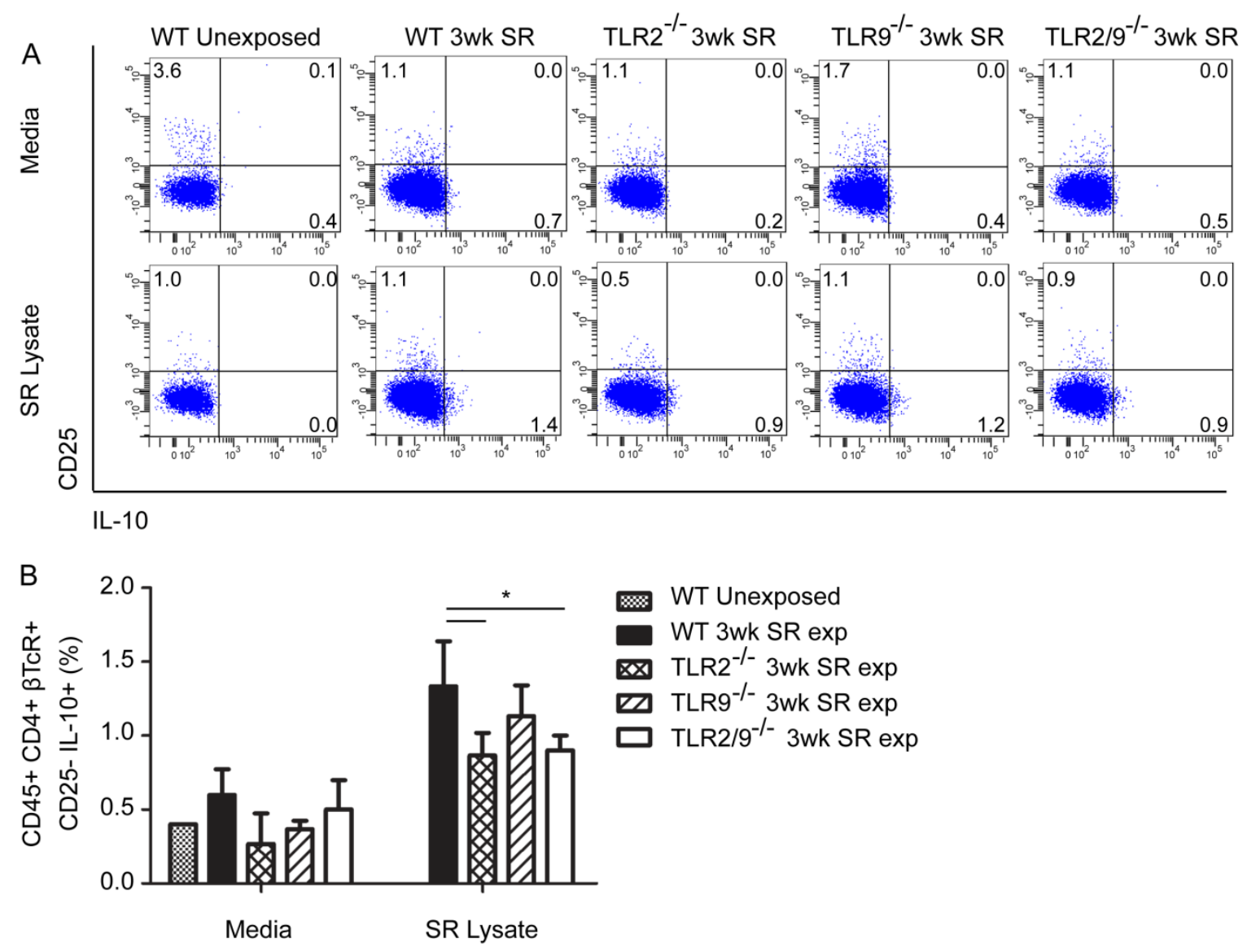

Figure 4-14. TLR2 is important for the generation of $\mathrm{IL}^{-10}$ expressing $\mathrm{CD}^{+} \mathrm{T}$ cells.

WT, TLR2 ${ }^{-/-}$, TLR9 ${ }^{-/-}$, and TLR $2 / 9^{-/-}$mice were exposed to SR 3 times/week for 3 weeks and analyzed on day 21 (4 days after the last exposure). Lung cells were digested and immune cells enriched on a 40/80\% percoll gradient were stimulated with SR lysate or media alone in the presence of brefeldin A then incubated with antibodies for CD45, $\beta T c R, C D 4, C D 25$, and IL-10 for intracellular cytokine staining. (A) Representative dot plots for $\mathrm{CD}^{+}$Th cells expressing CD25 or IL-10 (B) The percentage of CD25 $5^{-} \mathrm{CD} 4^{+}$ Th cells expressing IL-10. $(\mathrm{n}=3$ mice / group. The lung cells from 3 unexposed mice were combined.) Significance was determined using two-way ANOVA with Bonferroni post-hoc test $(* p<0.05$ compared to WT SR exposed mice). 
Figure 4-15. Reduced activation of $B$ cells in the lungs of TLR2/9 ${ }^{-/-}$mice.

WT, TLR2 ${ }^{-/-}$, TLR9 ${ }^{-/-}$, and TLR2 $/ 9^{-/-}$mice were exposed to SR 3 times/week for 3 weeks and analyzed on day 20 ( 3 days after the last exposure) or day 21 (4 days after the last exposure). Lung cells were digested and incubated with antibodies to CD45, $\beta$ TcR, CD8, CD19, CD69, and NK1.1 and analyzed by flow cytometry. (A) The percentage of B cells $\left(\mathrm{CD} 45^{+} / \mathrm{CD} 19^{+} / \mathrm{CD}^{-}\right)$and $\mathrm{B}$ cells that express CD69. (B) The percentage of $\mathrm{CD} 8^{+}$lymphocytes $\left(\mathrm{CD} 45^{+} / \mathrm{CD}^{+} / \mathrm{CD}^{-} 9^{-}\right)$and $\mathrm{CD}^{+}$lymphocytes that express CD69. (C) The percentage of NK cells $\left(\mathrm{CD} 45^{+} / \mathrm{NK} 1.1^{+} / \mathrm{CD} 19^{-}\right)$and $\mathrm{NK}$ cells that express CD69. ( $n=3-4$ mice per group. The lung cells from 3 or 4 unexposed mice were combined). The data represent mean $\pm \mathrm{SD}$ and significance was determined using oneway ANOVA with Tukey post-hoc test $(* p<0.05$ compared to WT SR exposed mice). 


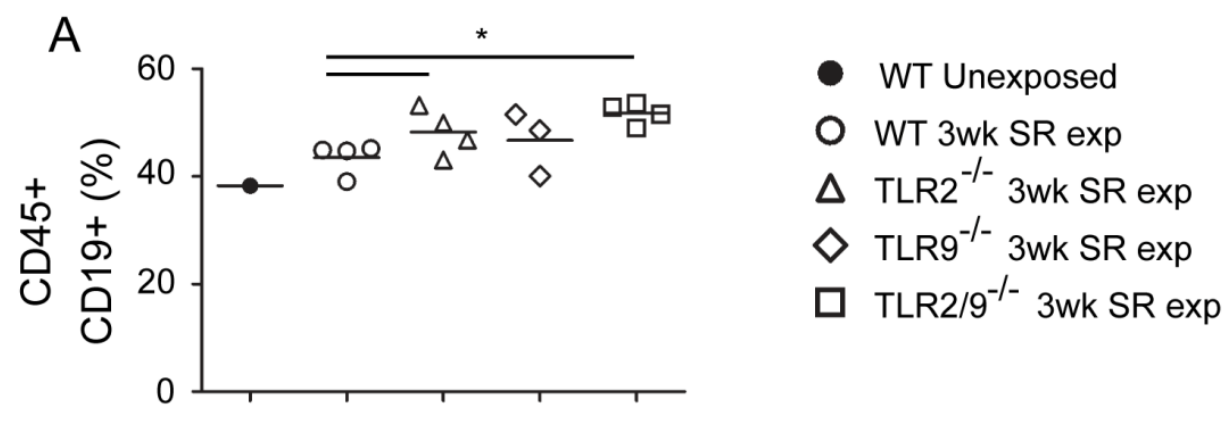

Day 20

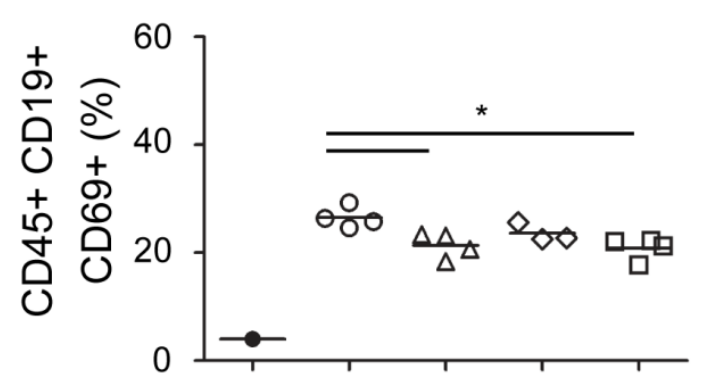

Day 20

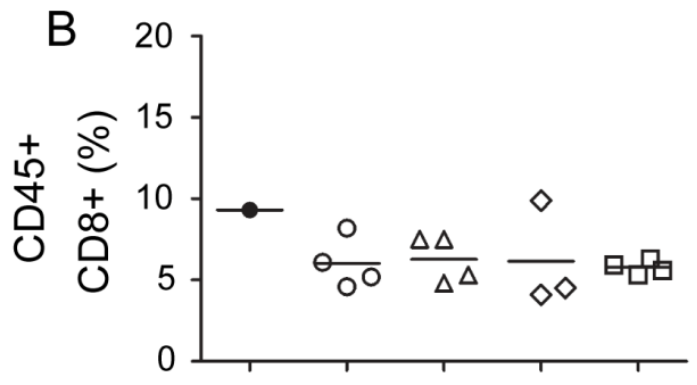

Day 20

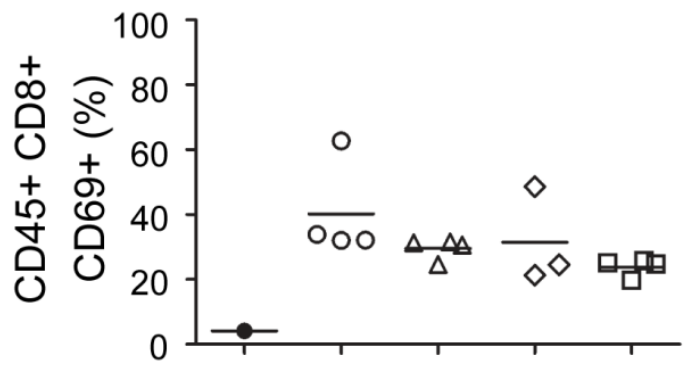

Day 20

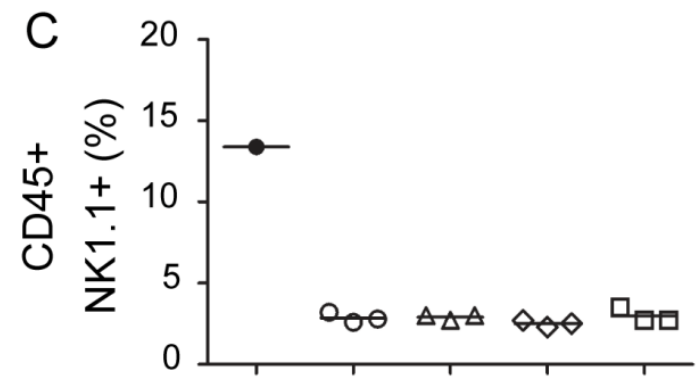

Day 21

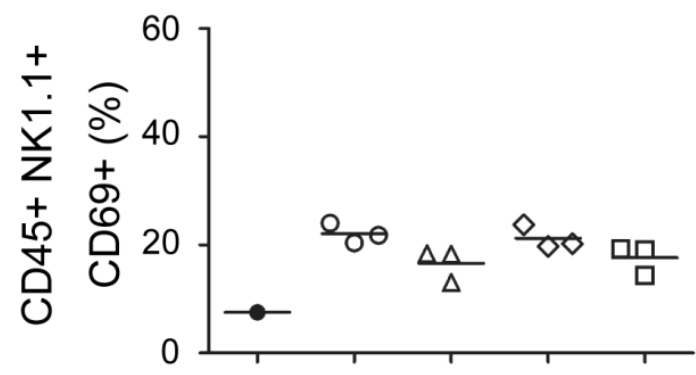

Day 21 
MHCII. In addition, $T$ cells can induce macrophage activation through stimulation of CD40 through CD40 ligand interaction. To determine whether macrophage activation was deficient in the SKO or TLR2/9 ${ }^{-/-}$mice, mice were exposed to SR 3 times a week for 3 weeks and sacrificed on day 18 ( 1 day after the last exposure) or day 21 ( 4 day after the last exposure) and co-stimulatory molecule and MHCII expression on macrophages isolated from whole lung tissue was measured by flow cytometry. Dispersed lung cells were incubated with antibodies to CD11b, F4/80, CD11c, Gr-1, CD80, CD86, MHCII, and CD40 and measured by flow cytometry. The gating strategy for the detection of interstitial macrophages $\left(\mathrm{CD} 11 \mathrm{~b}^{+} / \mathrm{F} 4 / 80^{+} / \mathrm{CD} 11 \mathrm{c}^{-} / \mathrm{Gr}-1^{-}\right)$is shown in Figure 4-16A.

We first examined costimulatory molecule CD80 and CD86 expression after 3 weeks exposure to SR. Interstitial macrophages had increased expression of CD80 on day 18 and day 21 after repeated SR exposure. TLR2 ${ }^{-/-}$, TLR9 ${ }^{-1-}$, and TLR $2 / 9^{-1-}$ mice had a significant decrease in the percentage of interstitial macrophages that expressed CD80. The geometric mean fluorescence intensity (MFI) was calculated, and showed the average intensity of CD80 staining was also significantly decreased in TLR2 ${ }^{-/-}$, TLR9 ${ }^{-/}$, and TLR2 $/ 9^{-/-}$cells on day 18 and day 21 (Figure 4-17A). The percentage of interstitial macrophages that expressed CD86 was also decreased in TLR2 ${ }^{-/-}$, TLR9 ${ }^{-/}$, and TLR2 $/ 9^{-/-}$mice on day 18 (Figure 4-17B). The results suggest TLRs 2 and 9 cooperate in the upregulation of costimulatory molecules CD80 and CD86 on interstitial macrophages.

MHCII was also upregulated on WT interstitial macrophages after 3 weeks exposure to SR. On average, $72 \%$ of the interstitial macrophages were $\mathrm{MHCII}^{\text {high }}$ on day 18 and 21 . TLR $9^{--}$, and TLR2 $/ 9^{-/-}$mice had a significant decrease in the percentage of MHCII $^{\text {high }}$ cells compared to WT SR exposed mice on day 18 (Figure 4-18A). The percentage of $\mathrm{MHCII}^{\text {high }}$ cells was also significantly decreased on day 21 in $\mathrm{TLR}^{-/-}$and TLR $2 / 9^{-1-}$ mice. In addition, the percent of interstitial macrophages that expressed CD40 was also significantly decreased in $\mathrm{TLR}^{-/-}$and TLR $2 / 9^{-/-}$mice compared to WT SR exposed mice (Figure 4-18B). The results suggest TLR9 is important for MHCII and CD40 upregulation on interstitial macrophages. Taken together, TLRs 2 and 9 appear to cooperate in the upregulation of CD80, CD86, MHCII, and CD40 on interstitial macrophages.

\section{Signaling Through TLR2 Inhibits Costimulatory Molecule Upregulation on Alveolar Macrophages (AMs)}

The lung also contains a macrophage population present in the alveoli of the lung responsible for immune surveillance and phagocytosis of microorganisms in the airways. To investigate the AM population after repeated SR exposure, mice were exposed to SR 3 times a week for 3 weeks and sacrificed on day 18 (1 day after the last exposure) or day 21 (4 day after the last exposure). To identify AMs, dispersed lung cells were incubated with antibodies to CD11c, CD205, F4/80, Gr-1, and CD11b and measured by flow cytometry. The gating strategy for the detection of AMs $\left(\mathrm{CD} 11 \mathrm{c}^{+} / \mathrm{CD} 205^{+} / \mathrm{F} 4 / 80^{+} /\right.$ 
A

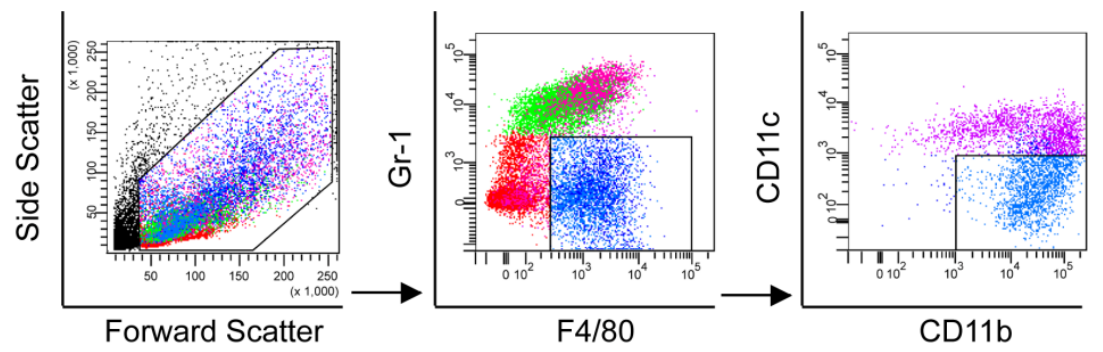

B
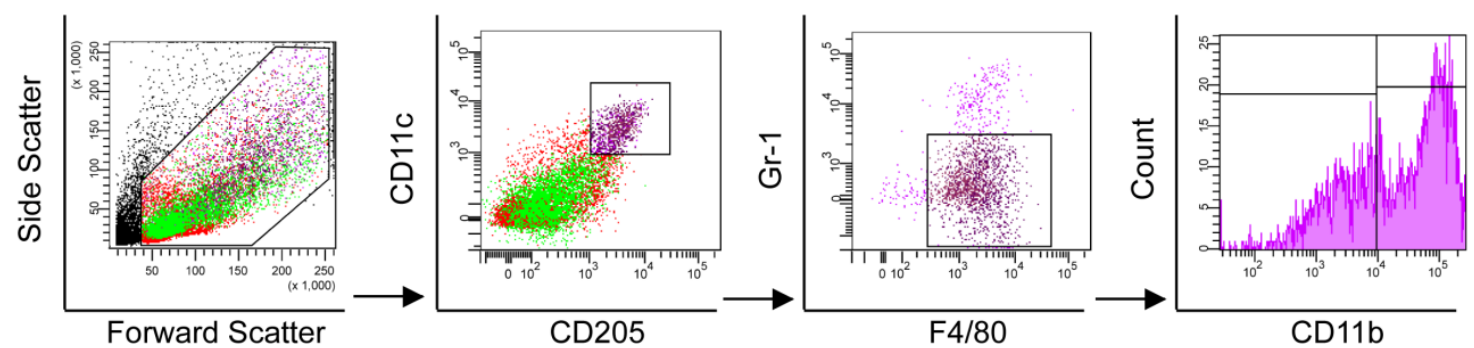

Figure 4-16. Gating strategy for the identification of interstitial and alveolar macrophages in a lung cell suspension.

WT, TLR2 ${ }^{-/-}$, $\mathrm{TLR}^{-/-}$, and TLR2 $/ 9^{-/-}$mice were exposed to SR 3 times/week for 3 weeks and analyzed on day 18 ( 1 day after the last exposure) or day 21 (4 days after the last exposure). Lung cells were digested and incubated with antibodies to CD11b, F4/80, Gr-1, CD11c, and CD205 and analyzed by flow cytometry. (A) Interstitial macrophages were identified as $\mathrm{F} 4 / 80^{+} / \mathrm{CD} 1 \mathrm{~b}^{+} / \mathrm{CD} 11 \mathrm{c}^{-} / \mathrm{Gr}-1^{-}$. (B) Alveolar macrophages (AMs) were identified as $\mathrm{CD} 11 \mathrm{c}^{+} / \mathrm{CD} 205^{+} / \mathrm{F} 4 / 80^{+} / \mathrm{Gr}-1^{-} / \mathrm{CD} 11 \mathrm{~b}^{\text {low }}$. In SR exposed mice, an

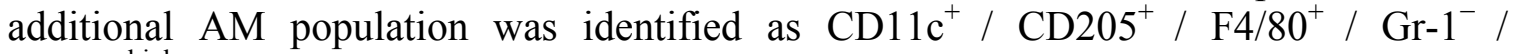
CD11b ${ }^{\text {high }}$. 
$\mathrm{F} 4 / 80+\mathrm{CD} 11 \mathrm{~b}+\mathrm{CD} 11 \mathrm{c}-\mathrm{Gr}-1-$

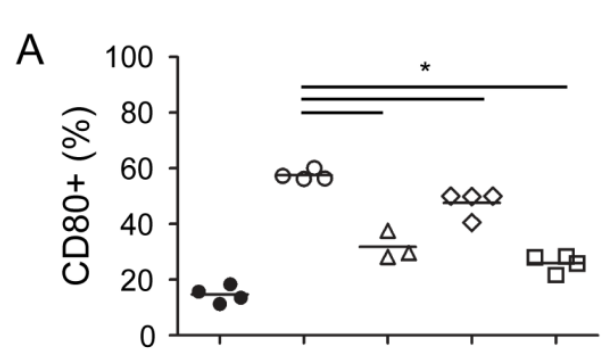

Day 18

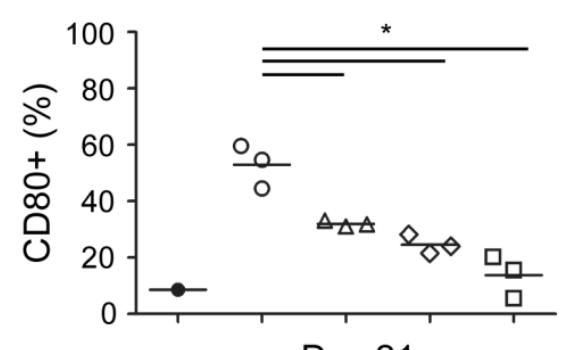

Day 21

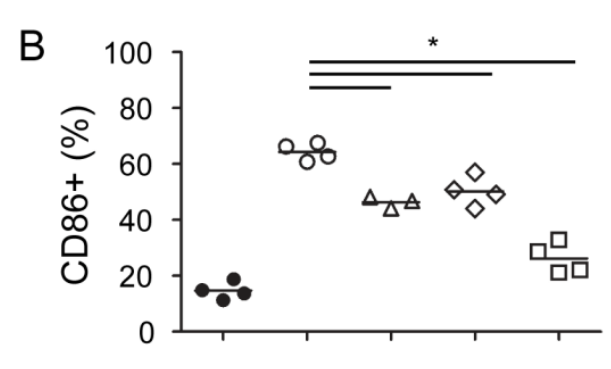

Day 18

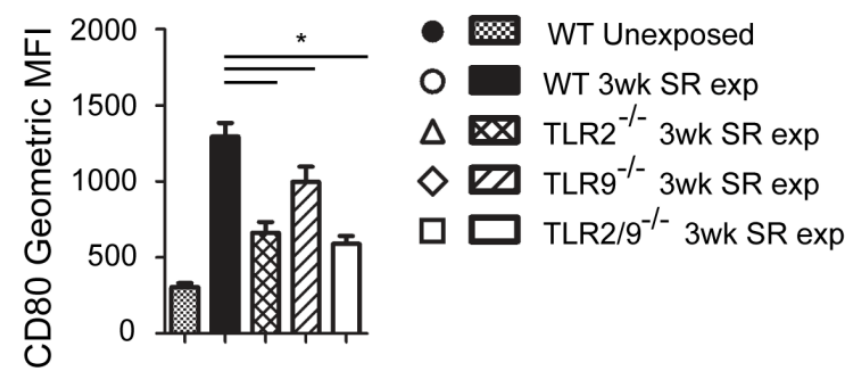

Day 18
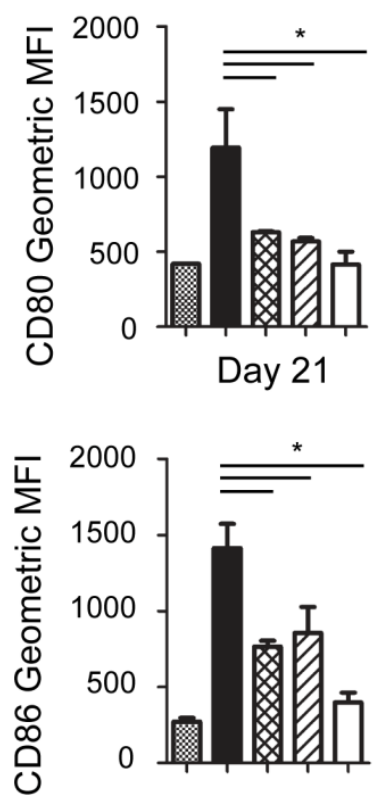

Day 18

Figure 4-17. TLRs 2 and 9 cooperate in CD80 and CD86 upregulation on interstitial macrophages after 3 weeks exposure to $S R$.

WT, TLR2 $2^{--}$, $\mathrm{TLR}^{-/-}$, and TLR2 $/ 9^{-/-}$mice were exposed to SR 3 times/week for 3 weeks and analyzed on day 18 ( 1 day after the last exposure) or day 21 (4 days after the last exposure). Lung cells were digested and incubated with antibodies to CD11b, F4/80, Gr-1, CD11c, CD80, and CD86 and analyzed by flow cytometry. Interstitial macrophages were identified as $\left(\mathrm{F} 4 / 80^{+} / \mathrm{CD} 1 \mathrm{~b}^{+} / \mathrm{CD} 11 \mathrm{c}^{-} / \mathrm{Gr}-1^{-}\right)$. The percentage of interstitial macrophages that express (A) CD80 and (B) CD86 and the geometric mean fluorescence intensity (MFI) was measured ( $n=3-4$ mice per group. On day 21, the lung cells from 3 unexposed mice were combined). The data represent mean $\pm \mathrm{SD}$ and significance was determined using one-way ANOVA with Tukey post-hoc test $(* p<0.05$ compared to WT SR exposed mice). 
$\mathrm{F} 4 / 80+\mathrm{CD} 11 \mathrm{~b}+\mathrm{CD} 11 \mathrm{c}-\mathrm{Gr}-1-$
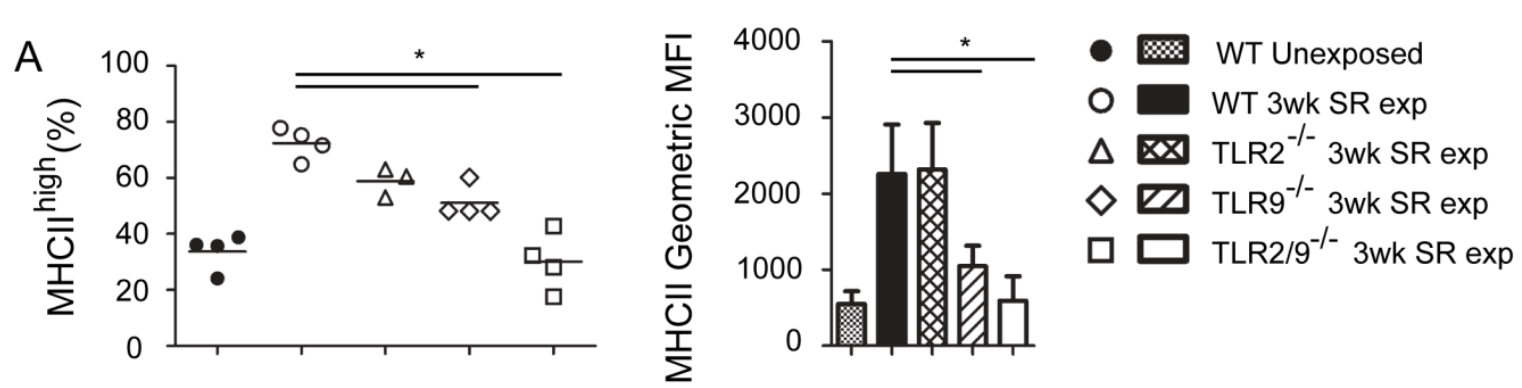

Day 18

\section{Day 18}
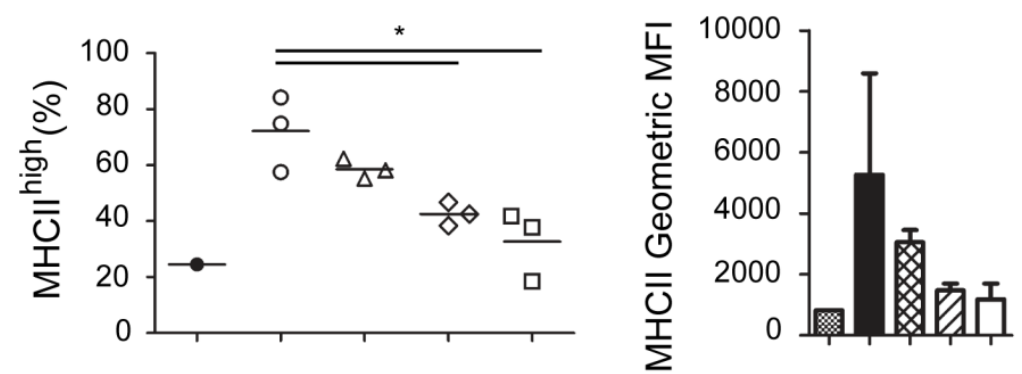

Day 21

Day 21

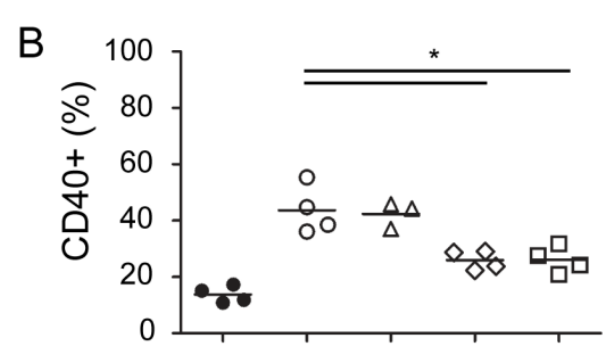

Day 18

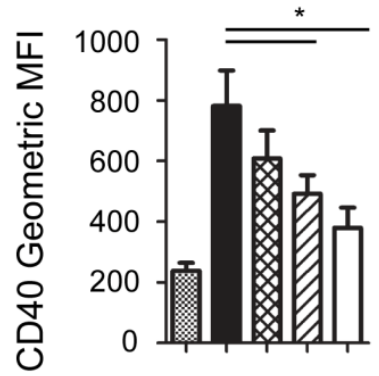

Day 18

Figure 4-18. TLR9 is required for MHCII and CD40 upregulation on interstitial macrophages after 3 weeks exposure to SR.

WT, TLR2 ${ }^{-/-}$TLR9 $^{-/-}$, and TLR2/9 ${ }^{-/-}$mice were exposed to SR 3 times/week for 3 weeks and analyzed on day 18 ( 1 day after the last exposure) or day 21 (4 days after the last exposure). Lung cells were digested and incubated with antibodies to CD1 lb, F4/80, Gr-1, CD11c, MHCII, and CD40 and analyzed by flow cytometry. Interstitial macrophages were identified as $\left(\mathrm{F} 4 / 80^{+} / \mathrm{CD} 1 \mathrm{~b}^{+} / \mathrm{CD} 1 \mathrm{c}^{-} / \mathrm{Gr}-1^{-}\right)$. The percentage of interstitial macrophages that express (A) MHCII and (B) CD40 and the geometric mean fluorescence intensity (MFI) was measured $(n=3-4$ mice per group. On day 21 , the lung cells from 3 unexposed mice were combined). The data represent mean $\pm \mathrm{SD}$ and significance was determined using one-way ANOVA with Tukey post-hoc test $(* p<0.05$ compared to WT SR exposed mice). 
Gr- $\left.1^{-} / \mathrm{CD} 11 b^{\text {low }}\right)$ is shown in Figure 4-16B. In SR exposed mice, an additional $\mathrm{CD} 11 \mathrm{~b}^{\text {high }} \mathrm{AM}$ population was detected, which was $\mathrm{CD} 11 \mathrm{c}^{+} / \mathrm{CD} 205^{+} / \mathrm{F} 4 / 80^{+} / \mathrm{Gr}-1^{-} /$ CD11b $b^{\text {high }}$. WT SR exposed mice had a greater percentage of CD11 $b^{\text {high }}$ AMs than CD11b ${ }^{\text {low }}$ AMs on day 18 and 21 (Figure 4-19). However, TLR2/9 ${ }^{-/-}$mice had a significantly greater percentage of CD11b ${ }^{\text {low }}$ AMs in the lung compared to WT SR exposed mice on day 18 . On day 21 , there was also a significantly greater percentage of CD11b ${ }^{\text {low }}$ AMs in TLR2 ${ }^{-/-}$mice as well as TLR2 $/ 9^{-/-}$mice compared to WT SR exposed mice. Therefore, TLR2 appears to play an important role in shifting the balance between $\mathrm{CD} 11 \mathrm{~b}^{\text {low }} \mathrm{AM}$ and $\mathrm{CD} 11 \mathrm{~b}{ }^{\text {high }} \mathrm{AM}$ populations in the lung after repeated SR exposure.

In healthy individuals AMs are usually in a quiescent state; however, AMs in patients with HP have increased costimulatory molecule expression (Israel-Assayag et al., 1999). Previous studies in mice have shown an increased percentage of CD11c cells that expressed CD86 and MHCII in the lung after exposure to SR and sendai virus, but only low levels when exposed to SR alone (Girard et al., 2009). To determine if loss of TLRs 2 and 9 alters costimulatory molecule and MHCII expression after repeated SR exposure, AMs were additionally stained with CD 80 and MHCII, and analyzed by flow cytometry. In WT SR exposed mice, the percentage of AMs that expressed CD80 was similar to unexposed mice on day 18 (Figure 4-20A). However, TLR $2^{-/}$and TLR $2 / 9^{-1-}$ mice had a significant increase in the percentage of AMs that expressed CD80. On day 21, the percentage of AMs that expressed CD80 was lower than day 18 , however TLR2/9 ${ }^{-/-}$still had a significant increase in the percentage of cells that expressed CD80. The results suggest signaling through TLR2 is important in inhibiting CD80 expression on AMs after SR exposure.

The expression of MHCII was also measured on AMs. On day 18 , TLR2 ${ }^{-/-}$mice had a significant increase in the percentage of AMs that expressed MHCII ${ }^{\text {high }}$ compared to WT SR exposed mice (Figure 4-20B). In contrast, TLR9 ${ }^{-/}$mice had a significant decrease in the percentage of AMs that expressed $\mathrm{MHCII}^{\text {high }}$ and the geometric MFI. However, there was no significant difference in TLR $2 / 9^{-/-}$mice compared to WT exposed mice. On day 21, there is no significant difference in the percentage of AMs that express MHCII ${ }^{\text {high }}$ in KO mice compared to WT exposed mice, suggesting the increase in MHCII expression was transitory. Altogether, the results suggest signaling through TLR2 is important for inhibiting MHCII expression on AMs. In addition, signaling through TLR9 may be important for MHCII upregulation as was seen in interstitial macrophages.

The expression of CD80 and MHCII was also examined on the CD11 $\mathrm{b}^{\text {high }} \mathrm{AMs}$ in SR exposed mice on day 18 and 21. In contrast to the CD11b ${ }^{\text {low }}$ AMs, TLR2 $/ 9^{-/-}$cells had a significant decrease in the percentage of cells that expressed CD80 on day 18, and the geometric MFI of CD80 was significantly decreased in TLR2 $2^{-/}$as well as TLR2/9 $9^{-/-}$ mice compared to WT SR exposed mice (Figure 4-21A). By day 21, there was no significant difference in CD80 expression between WT and KO SR exposed mice, suggesting the upregulation of CD80 was delayed in TLR2 $2^{-/}$and TLR2/9 $9^{-1-}$ mice. The results suggest signaling through TLR2 is important for CD80 expression on CD11 $\mathrm{b}^{\text {high }}$ AMs after repeated SR exposure. Although CD80 expression was decreased, MHCII 


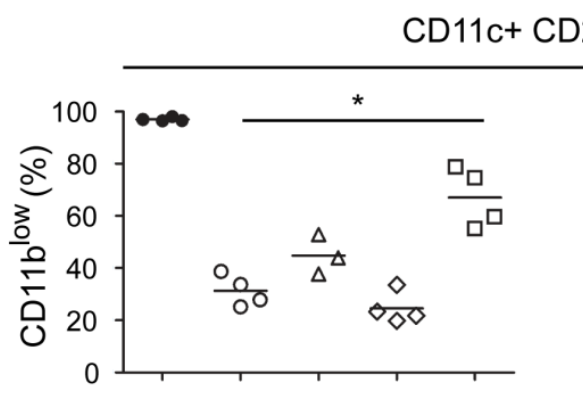

Day 18

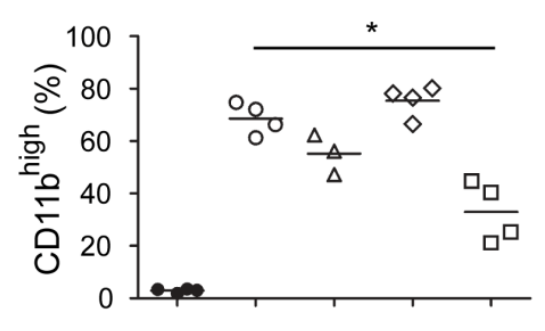

Day 18

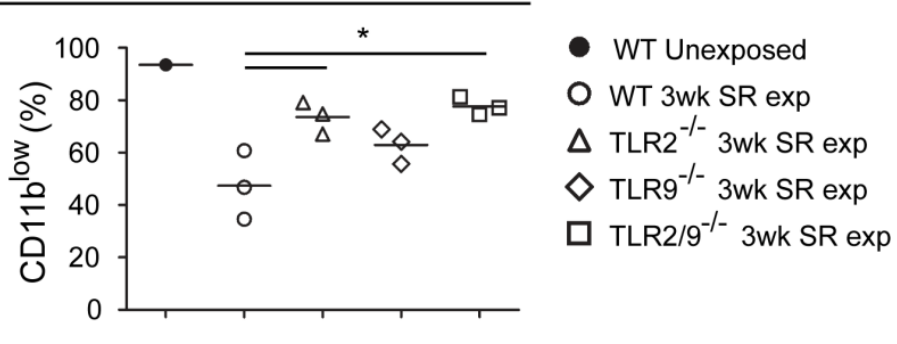

Day 21

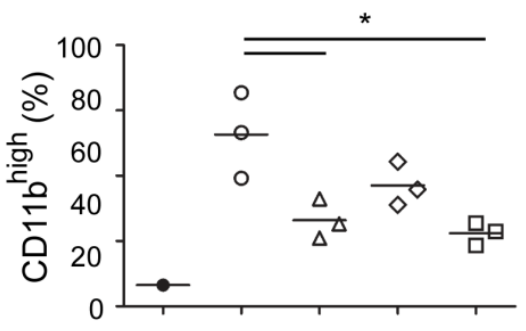

Day 21

Figure 4-19. TLR2 $/ 9^{-1}$ mice have a significant increase in the percentage of CD11b $^{\text {low }}$ AMs after 3 weeks exposure to SR.

WT, TLR2 ${ }^{-/-}$TLR9 $^{-/-}$, and TLR2 $/ 9^{-/-}$mice were exposed to SR 3 times/week for 3 weeks and analyzed on day 18 ( 1 day after the last exposure) or day 21 (4 days after the last exposure). Lung cells were digested and incubated with antibodies to CD11b, F4/80, Gr-1, CD11c, CD205 and analyzed by flow cytometry. AMs were identified as (CD11 $\mathrm{c}^{+} /$

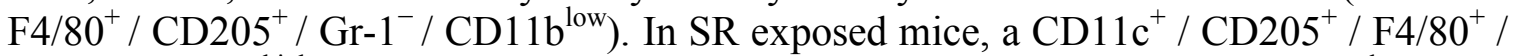
Gr-1 $1^{-} / \mathrm{CD} 11 \mathrm{~b}^{\text {high }} \mathrm{AM}$ population was also detected. The percentage of CD11 b ${ }^{\text {low }} \mathrm{AMs}$ and CD1 $1 b^{\text {high }}$ AMs in SR exposed mice $(n=3-4$ mice per group. On day 21, the lung cells from 3 unexposed mice were combined). The data represent mean $\pm \mathrm{SD}$ and significance was determined using one-way ANOVA with Tukey post-hoc test $\left({ }^{*} p<0.05\right.$ compared to WT SR exposed mice). 
Figure 4-20. Loss of TLR2 leads to upregulation of CD80 and MHCII on AMs after 3 weeks exposure to SR.

WT, TLR2 ${ }^{-/}$, TLR $^{-/-}$, and TLR2/ $9^{-/-}$mice were exposed to SR 3 times/week for 3 weeks and analyzed on day 18 ( 1 day after the last exposure) or day 21 (4 days after the last exposure). Lung cells were digested and incubated with antibodies to CD11b, F4/80, Gr-1, CD11c, CD205, and CD80 and analyzed by flow cytometry. (A) The percentage of AMs $\left(\mathrm{CD} 11 \mathrm{c}^{+} / \mathrm{F} 4 / 80^{+} / \mathrm{CD} 205^{+} / \mathrm{Gr}-1^{-} / \mathrm{CD} 11 \mathrm{~b}^{\text {low }}\right)$ that express $\mathrm{CD} 80$ and the geometric MFI was measured. (B) The percentage of AMs that express MHCII ${ }^{\text {high }}$ and the geometric MFI was measured. ( $n=3-4$ mice per group. On day 21, the lung cells from 3 unexposed mice were combined). The data represent mean $\pm \mathrm{SD}$ and significance was determined using one-way ANOVA with Tukey post-hoc test $\left(^{*} p<0.05\right.$ compared to WT SR exposed mice). 


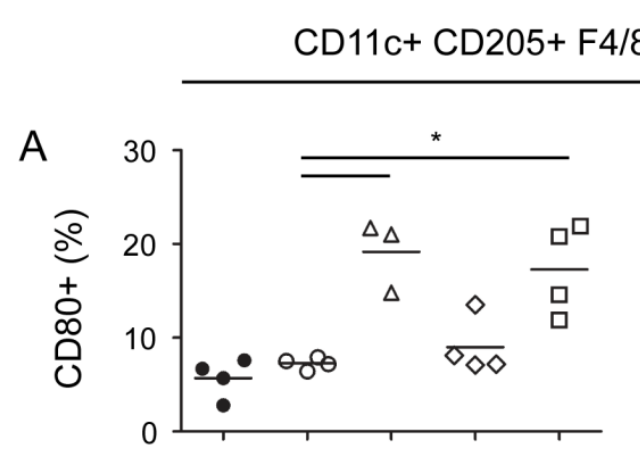

Day 18
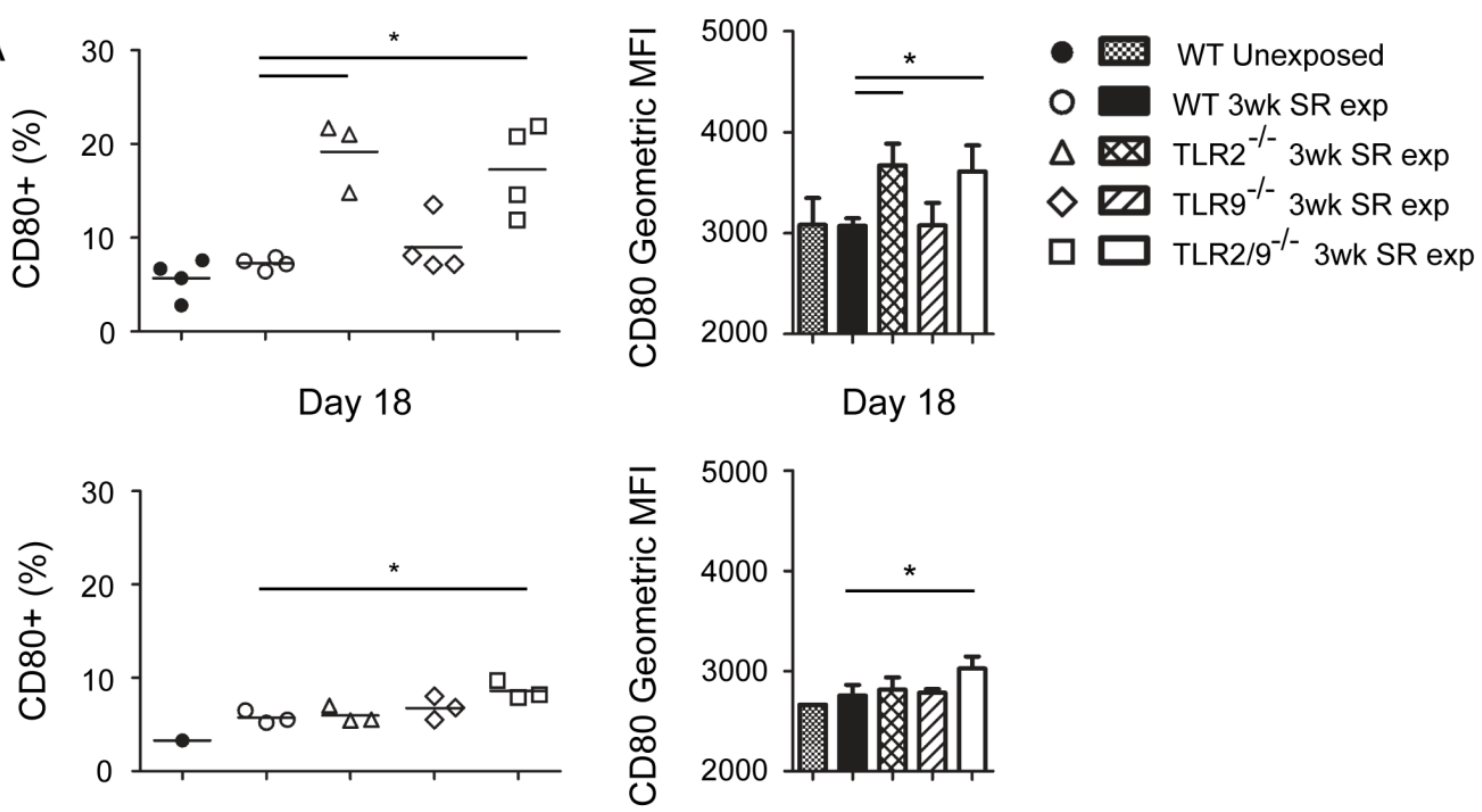

Day 21

Day 21

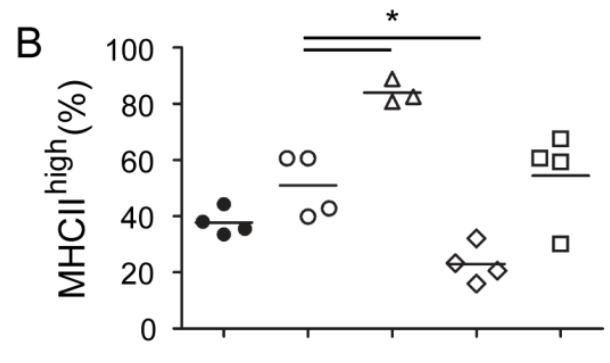

Day 18

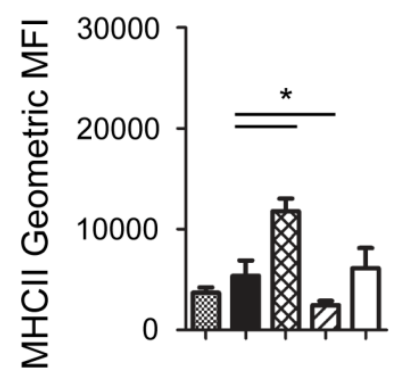

Day 18

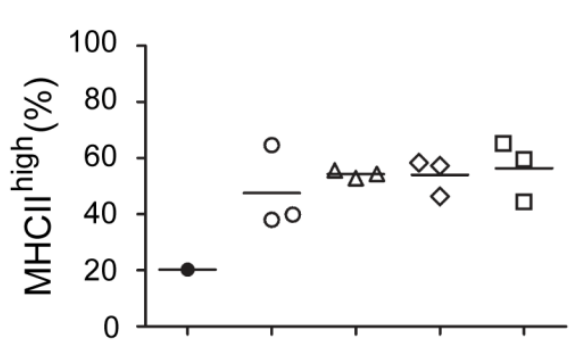

Day 21

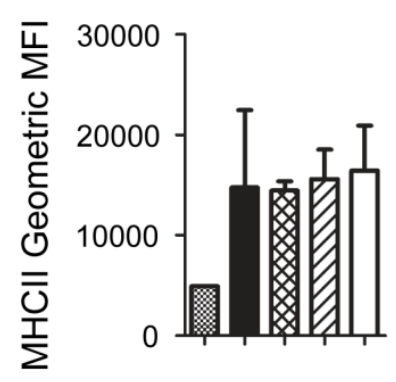

Day 21 
Figure 4-21. Loss of TLR2 leads to downregulation of CD80 on CD11b ${ }^{\text {high }}$ AMs after 3 weeks exposure to SR.

WT, TLR2 ${ }^{-/-}$, TLR9 ${ }^{-/-}$, and TLR2 $/ 9^{-/-}$mice were exposed to SR 3 times/week for 3 weeks and analyzed on day 18 ( 1 day after the last exposure) or day 21 (4 days after the last exposure). Lung cells were digested incubated with antibodies to CD11b, F4/80, Gr-1, CD11c, CD205, and MHCII and the cells analyzed by flow cytometry. (A) In SR exposed mice, the percentage of CD11 bigh $\mathrm{AMs}\left(\mathrm{CD} 11 \mathrm{c}^{+} / \mathrm{F} 4 / 80^{+} / \mathrm{CD} 205^{+} / \mathrm{Gr}-1^{-} /\right.$ $\mathrm{CD} 11 \mathrm{~b}^{\text {high }}$ ) that express CD80 and the geometric mean fluorescence intensity (MFI) was measured. (B) In SR exposed mice, the percentage of CD11b ${ }^{\text {high }}$ AMs that express $\mathrm{MHCII}^{\text {high }}$ and the geometric mean fluorescence intensity (MFI) was measured ( $n=3-4$ mice per group. On day 21, the lung cells from 3 unexposed mice were combined). The data represent mean \pm SD and significance was determined using one-way ANOVA with Tukey post-hoc test ( ${ }^{*} p<0.05$ compared to WT SR exposed mice). 


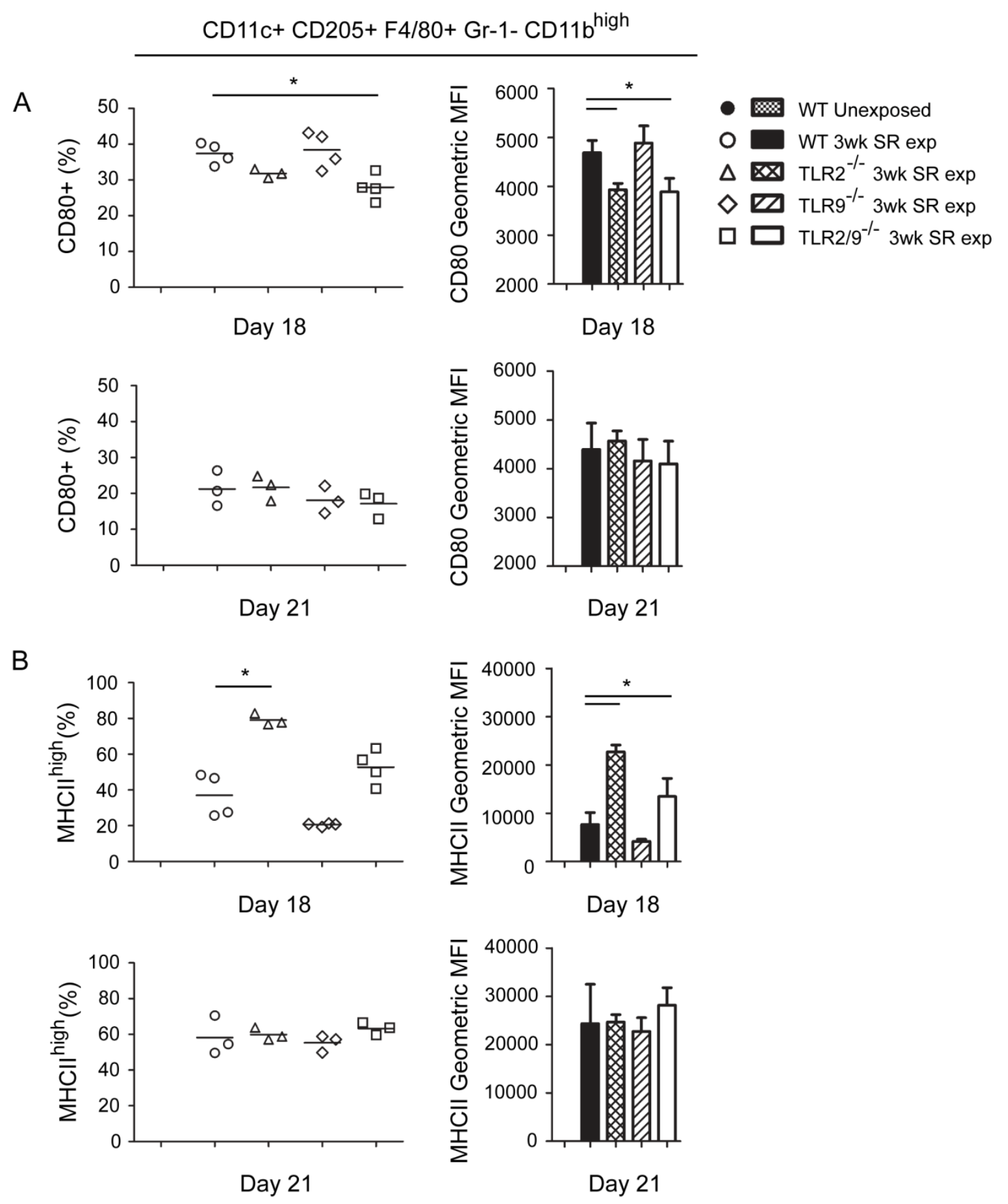




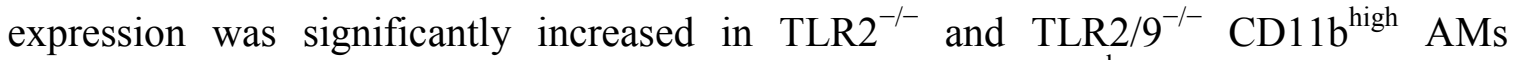
compared to WT SR exposed mice similarly to $\mathrm{CD} 11 \mathrm{~b}^{\text {low }}$ AMs on day 18 (Figure 4-21B). However on day 21, there was no significant difference in MHCII expression in WT and KO SR exposed groups suggesting the upregulation of MHCII was accelerated in $\mathrm{TLR}^{-/-}$and TLR $2 / 9^{-/-}$mice. Altogether, the results suggest signals through TLR2 are also important for inhibiting MHCII expression on CD11 $\mathrm{b}^{\text {high }}$ AMs.

\section{TLRs 2 and 9 Are Not Required for Granuloma Formation}

Cellular interactions through receptor binding and cytokine stimulation between antigen presenting cells and $\mathrm{T}$ cells are crucial for granuloma formation. TLR2 $/ 9^{-/-}$mice have significant reductions in inflammatory cytokine expression, $\mathrm{T}$ cell activation, the Th17 response, and MHCII and costimulatory molecule expression on interstitial macrophages. To determine if loss of TLRs 2 and 9 also results in reduced granuloma formation, mice were exposed to SR 3 times a week for 3 weeks and sacrificed on day 20 ( 3 days after the last exposure). The left lung lobe was harvested, fixed in formalin, and paraffin embedded sections were stained with $\mathrm{H} \& \mathrm{E}$ (Figure 4-22A-E). Wild type mice exposed to SR displayed peribronchiolar areas of granuloma formation while unexposed mice exhibited normal lung architecture (Figure 4-22A and B). The TLR2 ${ }^{-/}$, TLR9 $9^{-/}$ and TLR2 $/ 9^{-1-}$ mice exposed to SR all demonstrated granuloma formation (Figure 4-22C-E). The results suggest loss of TLRs 2 and 9 is not sufficient to inhibit granuloma formation in SR exposed mice. This suggests that additional PRRs must be involved in granuloma formation in HP.

\section{Discussion}

Initial studies during the acute phase of HP showed the alveolitis after a single SR exposure was predominantly neutrophilic, and neutrophil recruitment was dependent on TLRs 2 and 9. After 3 weeks repeated SR exposure, the alveolitis is comprised of neutrophils, monocytes, and lymphocytes. At this time point, TLR2/ $9^{-/-}$mice still have significant reductions in neutrophil recruitment, as well as neutrophil chemokine production, with TLR2 being the dominant receptor for CXCL2 expression. The reduction in neutrophil recruitment in TLR $2 / 9^{-/-}$mice was offset by a significant increase in $\mathrm{CD}_{11 \mathrm{~b}^{+}} \mathrm{Gr}-1^{-}$monocytes. Lymphocytes also make up a large percentage of the alveolitis. T cells and B cells were more numerous than $\gamma \delta \mathrm{T}$ cells or NK cells; however there was also no significant difference in the number of lymphocytes in WT or KO mice at this time point. Altogether the results suggest TLR2 is the dominant receptor for neutrophil recruitment with minor contributions from TLR9, but in the absence of TLRs 2 and 9, additional pattern recognition receptors can also contribute to monocyte and lymphocyte recruitment in response to repeated SR exposure.

Although the number of lymphocytes recruited to the airways was not significantly different between WT and KO SR exposed mice, there was a significant 


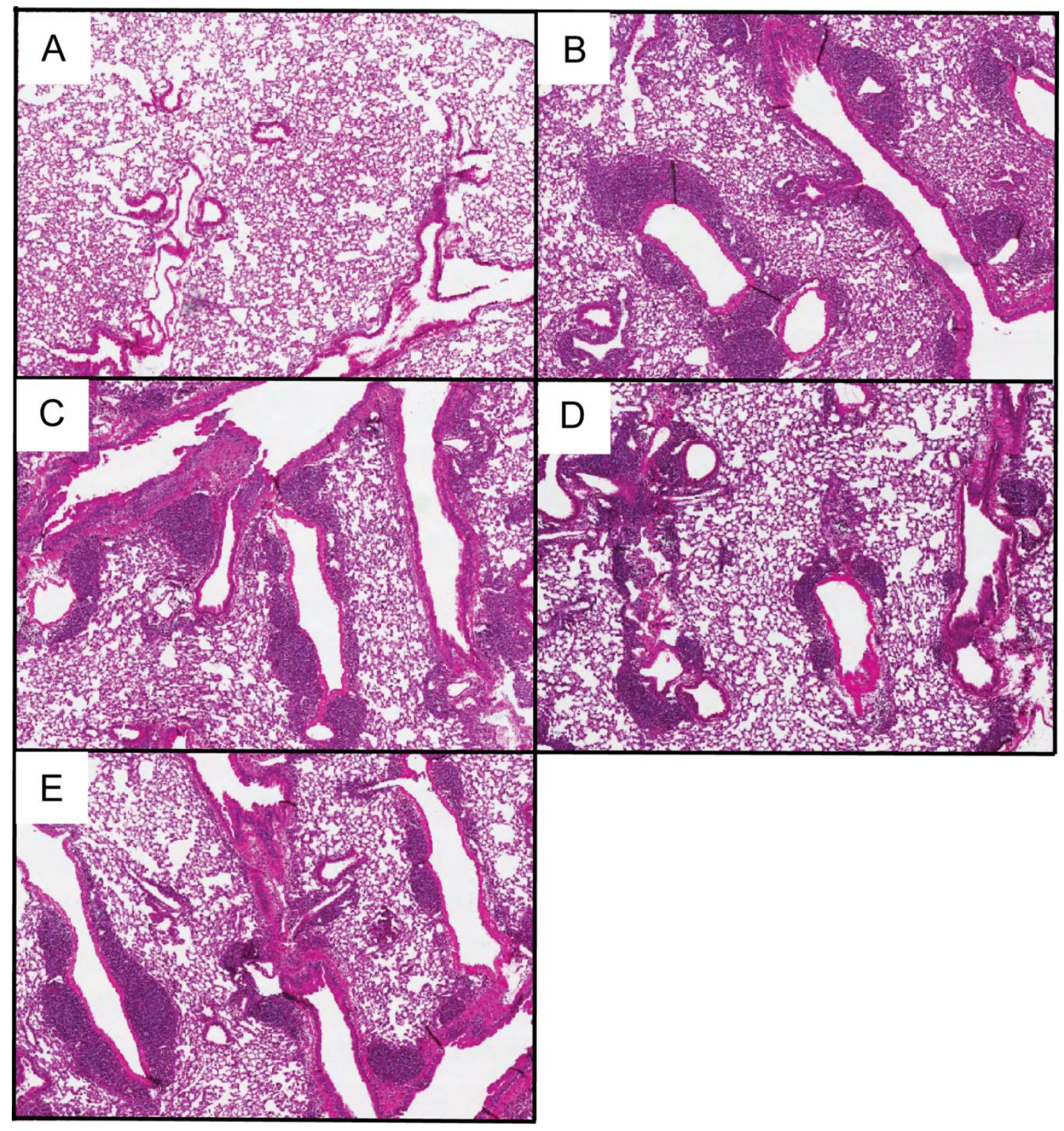

Figure 4-22. Granuloma development is not dependent on TLRs 2 and 9.

WT, TLR2 ${ }^{-/-}$, TLR9 ${ }^{-/-}$, and TLR $2 / 9^{-/-}$mice were exposed to SR 3 times/week for 3 weeks and analyzed 3 days after the last exposure. Representative H\&E stained lung sections from WT unexposed mice (A), WT SR exposed mice (B), TLR2 ${ }^{-/-}$SR exposed mice (C), TLR9 ${ }^{-/-}$SR exposed mice (D), and TLR2 $/ 9^{-/-}$SR exposed mice (E). (Original magnification $\mathrm{x} 4$ ). 
reduction in the percentage of $\mathrm{CD} 4^{+} \mathrm{T}$ cells in the lung of TLR2 ${ }^{-/-}$and TLR2/9 ${ }^{-/}$on day 20. There was no difference in the percentage of $\mathrm{CD}^{+}$Th cells that expressed CXCR3 or CCR6 between WT and KO mice, although we cannot rule out a reduction in percentage of Th cells that express other chemokine receptors. The expression of chemokines CCL2 and CCL4, which bind to CCR2 and CCR5 respectively, was reduced in TLR2 ${ }^{-1-}$ and TLR $2 / 9^{-/-}$mice, but not significantly different compared to WT SR exposed mice. However, the reduction in chemokine expression could contribute to the reduced percentage of $\mathrm{CD} 4^{+} \mathrm{T}$ cells in TLR2 $2^{--}$and TLR $2 / 9^{-/-}$mice.

The percentage of $\mathrm{CD}^{+} \mathrm{T}$ cells that expressed the activation marker CD69 was significantly reduced in TLR2 ${ }^{-/}$and TLR2 $/ 9^{-/}$mice the BAL and in the lung on day 20. This suggests signaling through TLR2 is important for T cell activation. Activation of naïve $\mathrm{CD}^{+} \mathrm{T}$ cells requires stimulation of the $\mathrm{T}$ cell receptor via antigen presented on MHCII, as well as CD28 stimulation through binding of costimulatory molecules CD80 and CD86. Effector memory $\mathrm{T}$ cells also require MHCII stimulation for activation. The expression of CD80 and CD86 on interstitial macrophages was significantly reduced in KO mice compared to WT after repeated SR exposure. MHCII expression was also significantly reduced in TLR $9^{-/}$and TLR $2 / 9^{-/-}$mice. Therefore, the reduced percentage of $\mathrm{CD}^{+} \mathrm{Th}$ in the lung after repeated SR exposures, as well as the reduced percentage of $\mathrm{CD}^{+}$Th cells expressing the activation marker CD69 could be due to the reduced capacity of antigen presenting cells to activate naïve $\mathrm{T}$ cells in TLR2 ${ }^{-/-}$and TLR $2 / 9^{-/-}$ mice.

However, naïve $\mathrm{T}$ cells require a third signal for activation through cytokine stimulation from antigen presenting cells. Previous studies revealed LPS induced MyD88-independent stimulation was sufficient for maturation of dendritic cell including upregulation of costimulatory molecules and migration to the draining lymph node, but that MyD88-dependent receptor induced upregulation of cytokine secretion was necessary to overcome Treg suppression of $\mathrm{T}$ cell activation (Pasare and Medzhitov, 2004). This suggests SR induced signaling through TLR2 may be important for the induction of cytokines in antigen presenting cells necessary for naïve $T$ cell activation. IL-6 was shown to be the critical cytokine stimulation required to overcome Treg suppression of naïve $\mathrm{T}$ cell activation (Pasare and Medzhitov, 2003). The expression of TNF $\alpha$ was significantly reduced in the lung in TLR2 ${ }^{-/-}$and TLR2 $/ 9^{---}$mice, but not IL-6, which may explain why $\mathrm{CD} 4{ }^{+} \mathrm{T}$ cell activation was reduced, but not absent.

IL-6 in combination with TGF $\beta$ leads to the polarization of Th17 cells in the mouse, while IL-23 induces proliferation and expansion of pathogenic Th17 cells (Bettelli et al., 2006; Mangan et al., 2006; McGeachy et al., 2007; Zhou et al., 2007). The Th17 response is generated after repeated SR exposure and is associated with increased lung severity and fibrosis (Joshi et al., 2009; Simonian et al., 2009), and previous studies have shown that the expression of Th17 cytokines was reduced in the absence of TLR6 (Fong et al., 2010). In this study, we found the expression of IL-17 was significantly reduced in $\mathrm{TLR}^{-/-}$, TLR9 ${ }^{-/-}$, and TLR $2 / 9^{-/-}$mice. IL-22, another Th17 associated cytokine was also significantly reduced in TLR2 $2^{-1-}$ and TLR $2 / 9^{-/-}$mice. 
The reduced IL-17 expression after 3 weeks exposure to SR correlated with a reduced percentage of Th17 cells in the lung in all KO mice compared to WT SR exposed mice on day 18, suggesting TLRs 2 and 9 contribute to the Th17 response. However, only TLR2 $/ 9^{-/-}$mice had a significant reduction in the percentage of Th17 cells in the lung on day 21 , suggesting TLR $2 / 9^{-/-}$are additionally deficient in maintaining the Th17 response. The Th17 cells from SR exposed mice secreted IL-17A but not IL-17F as was shown previously (Simonian et al., 2009). Studies during the acute phase showed TLR2/9 ${ }^{-/}$ mice were deficient in IL-23p19 expression, which could contribute to reduced Th17 proliferation in the lung; however there was no significant difference in the expression of IL-23p19 in WT and KO mice on day 18. The reduction in the Th17 response in TLR2 $/ 9^{-/-}$mice on day 21 was also not associated with a switch to a Th1 response.

$\mathrm{CD} 25^{+} \mathrm{FoxP}^{+}$Treg cells develop in the thymus and are important in controlling inflammatory responses, and previous studies have shown that $\mathrm{CD} 25^{+} \mathrm{CD} 4^{+}$regulatory cells are important for controlling IFN $\gamma$ producing T cells in HP (Park et al., 2009). A small percentage of $\mathrm{CD} 25^{+} \mathrm{FoxP}^{+} \mathrm{CD}^{+} \mathrm{T}$ cells were detected in the lung on day 21 , whereas the vast majority of FoxP $3^{+}$cells were $\mathrm{CD} 25^{-}$. Previous studies have shown $\mathrm{CD} 25^{-} \mathrm{CD}^{+} \mathrm{T}$ cells can be induced to upregulate FoxP3 and adopt a regulatory phenotype in the presence of TGF $\beta$ (Fantini et al., 2004; Fu et al., 2004). The increase in $\mathrm{CD} 25^{-}$FoxP3 $^{+} \mathrm{CD}^{+}$Treg cells suggests that induced Treg cells may play a more important role in inhibiting the immune response in the lung after SR exposure.

However, the presence of TNF $\alpha$ has been shown to inhibit the suppressive activity of Tregs by inducing dephosphorylation of FoxP3 (Nie et al., 2013; Valencia et al., 2006). TLR $2 / 9^{-/-}$mice have a significant decrease in TNF $\alpha$ expression in the lung, as well as decreased percentage of $\mathrm{CD} 25^{-} \mathrm{CD} 4^{+}$Th cells that express TNFa in response to SR lysate stimulation. Therefore, the reduction in the percentage of Th17 cells in TLR $2 / 9^{-/-}$mice may not be due to an increase in the percentage of natural or induced Treg cells, but instead the Treg cells may have an increased ability to suppress $\mathrm{T}$ cells responses in TLR $2 / 9^{-/-}$mice due to the significant decrease in TNF $\alpha$ in the lungs compared to WT SR exposed mice. In support of this hypothesis, previous studies have shown that Tregs isolated from patients with active HP and high concentrations of IL-17 and TNF $\alpha$ in the BALF are deficient in suppressing T cell proliferation ex vivo (Girard et al., 2011). Further studies are needed to test this hypothesis.

The reduction in $\mathrm{CD} 4^{+}$Th cells in TLR2 $2^{-/}$and TLR2/9 $9^{-/-}$mice was associated with an increase in the percentage of B cells in the lung on day 20 , and TLR2/9 ${ }^{-/-}$mice on day 21. Although not statistically significant, an increased number of B cells was also seen in the BAL on day 18 and day 20. However, the percentage of B cells that expressed the activation maker CD69 was significantly reduced in TLR2 ${ }^{-/-}$and TLR2 $/ 9^{-/-}$mice, suggesting signaling through TLR2 is important for activation of B cells. Further experiments are necessary to establish if the reduced activation of B cells is due to loss of TLR2 on the B cell, or a result of the reduced activation of Th cells. B cells bind antigen through their B cell receptor and display antigen on MHCII. When $\mathrm{CD}^{+} \mathrm{Th}$ cells recognize the antigen displayed on MHCII, they stimulate B cells through CD40-CD40L interactions to enter germinal centers and undergo affinity maturation and class 
switching. Therefore, the reduction in the percentage of activated B cells in TLR $2^{-/}$and TLR $2 / 9^{--}$mice on day 20 could be due to the reduced percentage of activated CD $4^{+}$Th cells also seen on day 20 .

Alveolar macrophages are normally in a quiescent state in a healthy lung; however, in patients with HP, the expression costimulatory molecules increased (IsraelAssayag et al., 1999). WT mice did not significantly upregulate CD80 on AMs compared to unexposed mice; however, TLR2 $2^{--}$and TLR $2 / 9^{-/}$mice had increased expression of CD80 on AMs on day 18 , and CD80 expression remained elevated on TLR2/9 ${ }^{-/-}$mice on day 21. This suggests signaling through TLR2 is required to inhibit costimulatory molecule expression on AMs. SR exposed mice had an increase in the percentage of CD1 $1 b^{\text {high }}$ AMs, and this macrophage population was significantly reduced in TLR $2 / 9^{-/-}$ mice. CD11 $b^{\text {high }}$ AMs have been reported in patients with HP, as well as sarcoidosis, and idiopathic pulmonary fibrosis (Krombach et al., 1996). Previous studies have suggested CD11b is important for the recruitment of AMs into the lung after Streptococcus pneumoniae challenge (Kirby et al., 2006). In contrast to the CD11b ${ }^{\text {low }}$ AMs, the expression of costimulatory molecule CD80 was significantly reduced on the CD11 $\mathrm{b}^{\text {high }}$ AMs in TLR2 $/ 9^{-/-}$mice suggesting TLRs 2 and 9 are required for CD80 upregulation in this cell type. MHCII was upregulated in TLR2 $2^{-/}$and TLR $2 / 9^{-/-}$mice on day 18 on AMs, as well as CD11b ${ }^{\text {high }}$ AMs, but there was no difference between groups on day 21 , suggesting loss of TLR2 accelerates MHCII upregulation. Altogether, the results suggest repeated SR exposure leads increased MHCII expression on AMs, and costimulatory molecule upregulation on CD11 $b^{\text {high }} \mathrm{AMs}$, as has been observed in patients; but loss of signaling through TLR2 leads to increased CD11b ${ }^{\text {low }}$ AM activation. Previous studies have shown increased expression of CD86 on CD11 ${ }^{+}$cells in the lung after sendai virus infection and exposure to SR, which supports the authors hypothesis that prior viral infection leads to increased immune responses to HP antigens (Girard et al., 2009; IsraelAssayag et al., 1999). TLR-induced immune responses have been shown to be inhibited following viral infections (Didierlaurent et al., 2008; Oliver et al., 2008), which is associated with increased risk of secondary bacterial infection. Further research into the relationship between viral infection and subsequent TLR induced SR sensitization is needed.

Three weeks exposure to SR resulted in increased interstitial macrophage activation as evidenced by an increased percentage of interstitial macrophages that expressed CD80, CD86, CD40, and high levels of MHCII. Mice deficient in TLR2 or TLR9 had a reduced percentage of interstitial macrophages that expressed CD80 or CD86, which was further reduced in TLR2/ $9^{-/-}$mice, suggesting TLRs 2 and 9 cooperate

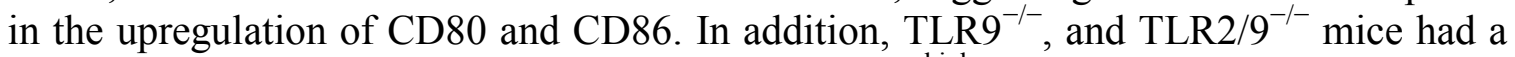
significant reduction in the percentage of $\mathrm{MHCII}^{\text {high }}$ and CD40 expressing cells. Altogether, the results suggest signaling through TLRs 2 and 9 cooperate in interstitial macrophage activation in response to SR exposure. Classically activated macrophages as well as regulatory macrophages require TLR stimulation for activation (Edwards et al., 2006). Further experiments would be necessary to determine if repeated SR exposure leads to classically activated macrophages typified by inflammatory cytokine secretion and the upregulation of nitric oxide synthase, regulatory macrophages identified by IL-10 
secretion, or if in the absence of TLR 2 and 9 signaling, SR exposure induces an alternatively activated macrophage phenotype.

Granuloma formation depends on the interaction between $\mathrm{T}$ cells and macrophages through physical contact as well as cytokine signals. TLR $2 / 9^{-/-}$mice had reduced $\mathrm{T}$ cell activation, cytokine expression, as well as interstitial macrophage costimulatory molecule expression and MHCII upregulation, which suggested TLR2/9 ${ }^{-/-}$ may also be deficient in granuloma formation. However, TLR2 ${ }^{-/-}, \mathrm{TLR}^{-/-}$, and TLR2 $/ 9^{-/}$mice formed granulomas similarly to WT SR exposed mice. Further experiments would be necessary to determine if granulomas resolve faster, or if the cellular composition of the granuloma is altered in TLR2 ${ }^{-/}$, TLR $9^{-/}$, or TLR2 $/ 9^{-/-}$mice.

In summary, mice deficient in TLRs 2 and 9 are partially protected from SR induced HP. TLR2 $/ 9^{-/-}$mice have a significant reduction in neutrophils at this time point, as well as a significant reduction in activated $\mathrm{CD} 4^{+} \mathrm{Th}$ cells, two cell types that have been associated with increased lung pathology. In addition, TLR2 $/ 9^{-/-}$have a significant reduction in the percentage of Th17 cells after three weeks SR exposure, a cell type that has been associated with lung fibrosis in HP. In the next chapter, we will explore whether TLR2 $/ 9^{-/-}$mice are protected from developing chronic HP. 


\section{CHAPTER 5. CHRONIC PHASE}

\section{Introduction}

Chronic HP (CHP) is associated with emphysema and a restrictive lung disease characterized by fibrosis and reduced lung compliance (Costabel et al., 2012; ErkinjunttiPekkanen et al., 1998). CHP is progressive and irreversible and is associated with high morbidity and mortality (Hanak et al., 2008; Vourlekis et al., 2004). At this stage, antigen avoidance will not resolve symptoms but can prolong survival (Fernandez Perez et al., 2013). Deaths where HP is the underlying or contributing cause have steadily increased from 1979 to 2007 (NIOSH., 2012). However little is known about the transition between HP and CHP. One hypothesis suggests the transition to progressive fibrosis in CHP is associated with a switch to a Th2 response. A study which looked at $\mathrm{T}$ cell responses in patients with subacute and chronic pigeon breeder's disease, another common type of HP, found an increase in $\mathrm{CD}^{+}$and $\mathrm{CD} 8^{+} \mathrm{T}$ cells expressing IL-4 and a decrease in T cells expressing IFN $\gamma$ in patients with CHP compared to subacute HP (Barrera et al., 2008). This is in agreement with a mouse model of chronic pigeon breeder's disease in which the authors found that IL-4 / IL-13 expression and a Th2-biased immune response correlated with increased lung fibrosis (Mitaka et al., 2011). Fibrotic diseases are often associated with the expression of Th2-associated cytokines IL-4 and IL-13 (GharaeeKermani et al., 2001; Huaux et al., 2003; Jakubzick et al., 2003; Lee et al., 2001). Wound healing alternatively activated macrophages, which are polarized in the presence of IL-4, have been shown to be both pathogenic and protective in fibrosis development (Gibbons et al., 2011; Pesce et al., 2009). However, previous studies have shown IL-4 and Th2biased immune responses are associated with reduced disease severity in SR induced HP (Butler et al., 2002; Gudmundsson et al., 1998; Jimenez-Alvarez et al., 2010; Matsuno et al., 2007). Therefore, further examination of Th2 immune responses during SR induced CHP is necessary.

Neutrophils have also been associated with lung fibrosis in CHP. There is a correlation between the level of neutrophils in the lung tissue and severity of fibrosis in patients with CHP (Pardo et al., 2000). The neutrophils expressed high levels of gelatinase B and collagenase 2, which contribute to lung damage. The IL-17 response is associated with the induction of neutrophil chemokines, neutrophil recruitment in the lung (Hellings et al., 2003; Laan et al., 1999; Miyamoto et al., 2003; Witowski et al., 2000). Other models of lung fibrosis such as bleomycin and IL-1-mediated lung fibrosis have also found IL-17 production by Th17 and $\gamma \delta \mathrm{T}$ cells is associated with increased lung fibrosis (Wilson et al., 2010). In addition, recent studies in mice have shown that neutrophils and macrophages were the main cell type that expressed IL-17A in HP and that neutrophil depletion decreased the amount of collagen in the lungs after SR exposure (Hasan et al., 2013). However, the role of IL-17 in fibrosis is controversial. A mouse model of experimental silicosis found that IL-17 produced by $\mathrm{CD} 4{ }^{+} \mathrm{T}$ cells and $\gamma \delta \mathrm{T}$ cells was important for early inflammation and neutrophil recruitment, but IL-17 and signaling through the IL-17R was dispensable for TGF $\beta$ production and fibrosis development at later time points (Lo Re et al., 2010). 
Studies during the acute and granulomatous phase show TLR2/9 $9^{-/}$mice have significant reductions in neutrophil recruitment, IL-17 expression, and the Th17 response. We hypothesize the reduced neutrophil recruitment and Th17 response in TLR2/ $/ 9^{-/-}$mice will protect against the development of fibrosis during CHP. In this chapter, we will examine the immune response that develops in a mouse model of SR induced CHP to determine if mice switch from a Th17 to a Th2 response. In addition, we will measure lung compliance and fibrosis to determine if loss of TLRs 2 and 9 protect against the development of interstitial lung disease after long term exposure to SR.

\section{Results}

\section{Mice with Long Term Exposure to SR Develop a Neutrophilic and Lymphocytic Alveolitis}

To measure the cellular composition of the alveolitis in mice with CHP, mice were intranasally exposed to SR 3 times/week for 15 weeks, and BAL was performed 3 days after the last exposure. BAL cells were spun onto slides and stained for differential counting (Figure 5-1). WT and TLR2/9 $9^{-/}$mice exposed to SR for 15 weeks had a significant increase in the percentage of neutrophils compared to unexposed mice. Interestingly, TLR $2 / 9^{-/-}$mice also had a significant increase in eosinophils compared to WT unexposed mice and SR exposed mice. However, neutrophils were still the dominant cell type in the alveolitis, as the percentage of eosinophils accounted for no more than $6 \%$ of the total BAL cells in TLR $2 / 9^{--}$mice and less than $1 \%$ in WT SR exposed mice. The increase in the percentage of neutrophils was associated with a significant increase in the expression of the neutrophil chemokine CXCL2 mRNA in the lung tissue of 15 week SR exposed mice compared to unexposed mice (Figure 5-2A). The increase in eosinophils in TLR2 $/ 9^{-/-}$mice was not associated with an increase in the expression of CCL11/eotaxin, a chemokine associated with eosinophil recruitment (Figure 5-2B). The percentage of lymphocytes was also significantly increased compared to unexposed mice (Figure 5-1). There was a decrease in the percentage of macrophages in the BAL cells of mice exposed to SR for 15 weeks compared to unexposed mice due to the influx of immune cells into the lungs. Monocytes and large multinucleated macrophages were also observed in SR exposed mice. The results suggest mice with long term exposure to SR develop a neutrophilic and lymphocytic alveolitis.

We next wanted to know if the composition of the alveolitis differs in mice exposed long term to SR versus short term. Mice were intranasally exposed to SR for 4 or 14 weeks and BAL performed 2 days after the last exposure. BAL cells were stained with antibody to CD11b, CD11c, Gr-1, F4/80, and $\beta$ TcR and immune cell populations were determined by flow cytometry. The gating strategy to discriminate neutrophils, AMs/macrophages, CD11 ${ }^{+}$DCs, and $\mathrm{T}$ cells is depicted in Figure 5-3A. There was no significant difference in the percentage of neutrophils or T cells in the BAL cells, however there was a significant increase in the percentage of AMs and a significant 

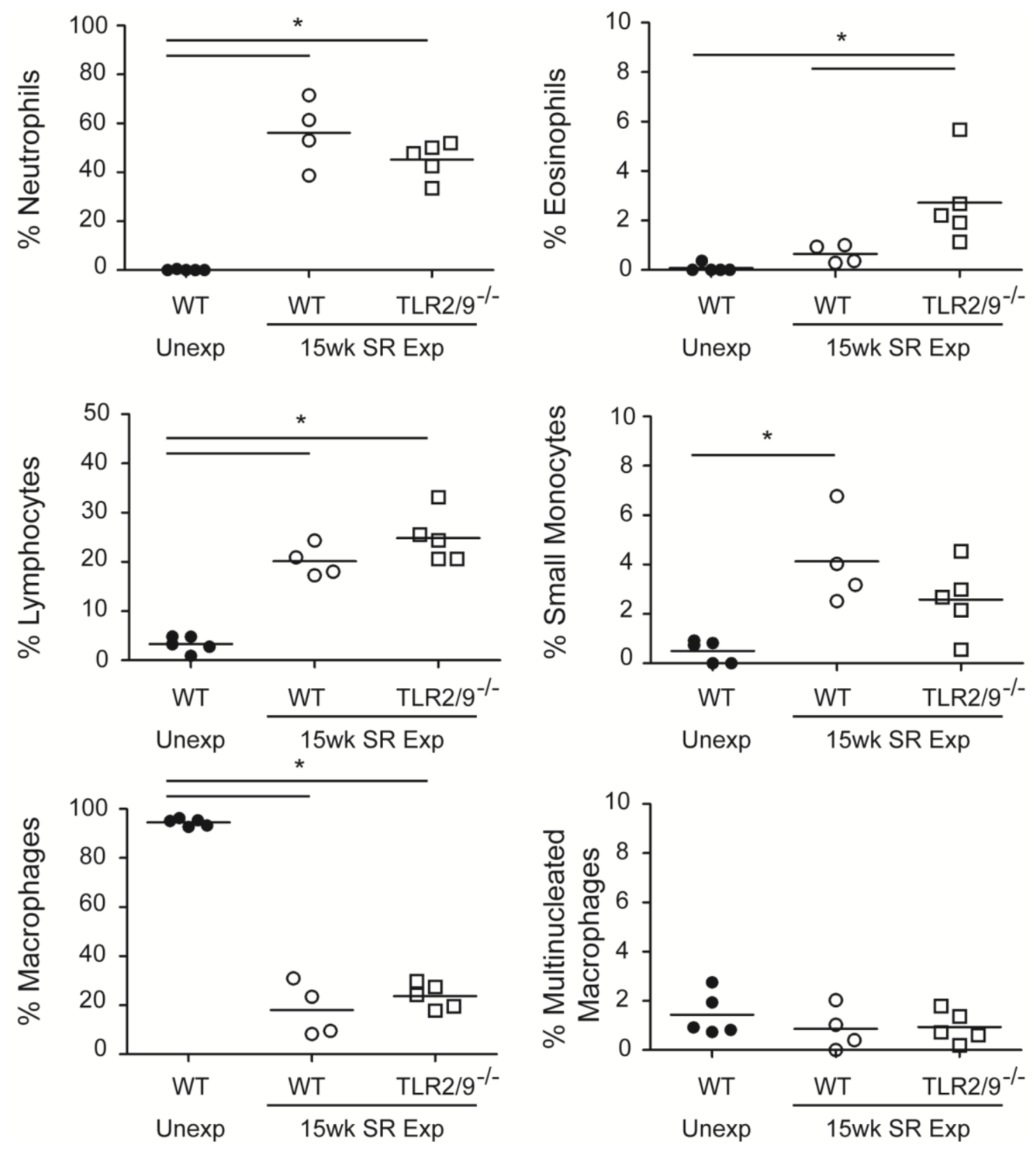

Figure 5-1. Composition of alveolitis in mice exposed to SR for 15 weeks.

WT and TLR2 $/ 9^{-/-}$mice were exposed to SR for 15 weeks and analyzed 3 days after the last exposure. BAL was performed and cells recovered from the BAL fluid spun onto slides and stained with a modified Wright Giemsa stain (Diff quick) for differential counting ( $n=4-5$ mice per group). A minimum of 4 areas at 20x were examined until a minimum of 200 cells were counted. The data represent mean \pm SD and significance was determined using one-way ANOVA with Tukey post-hoc test $\left({ }^{*} p<0.05\right)$. 

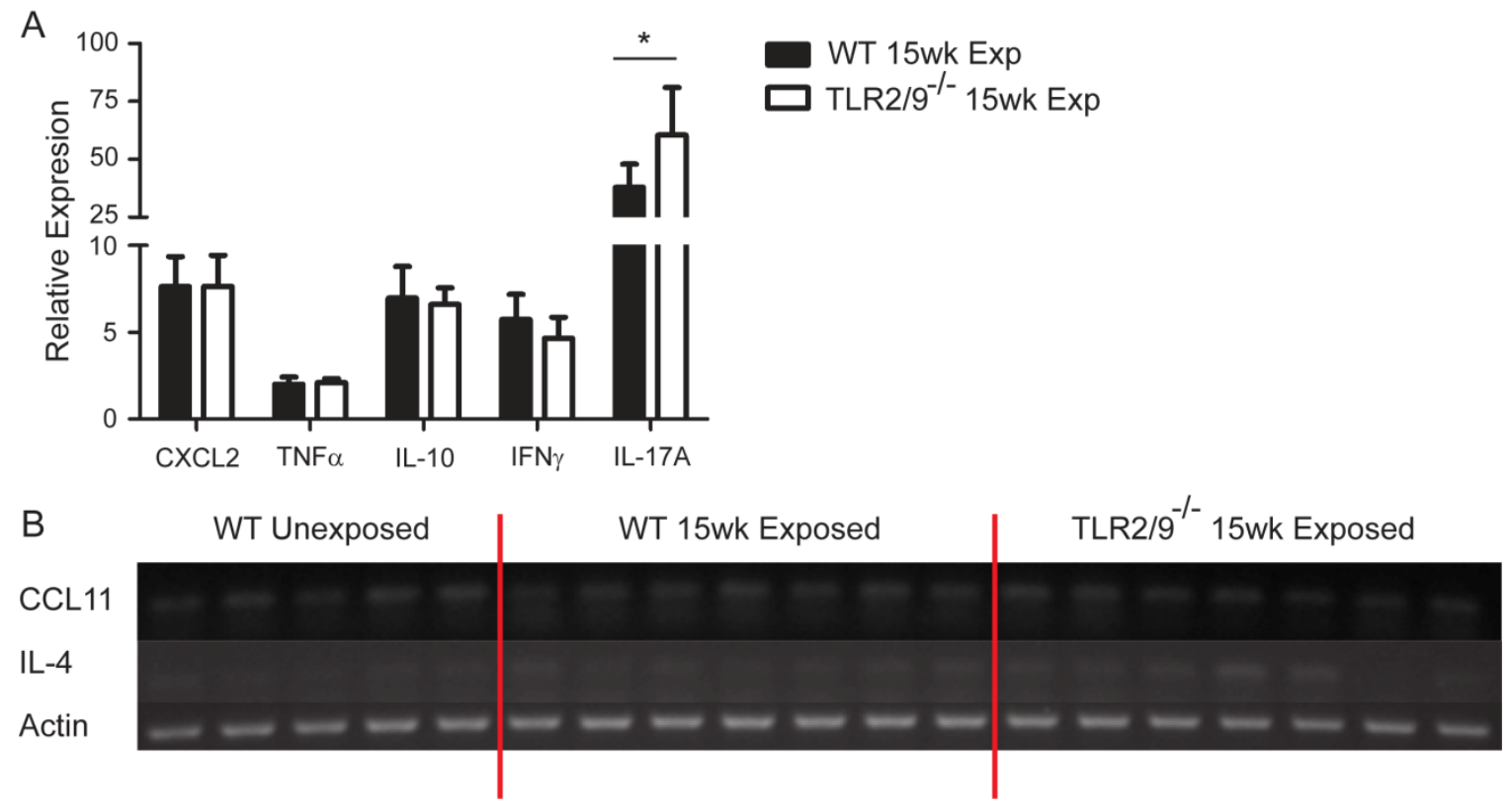

Figure 5-2. Th17-associated cytokine expression detected in the lungs of mice exposed to SR for 15 weeks.

WT and TLR2 $/ 9^{-/-}$mice were exposed to $150 \mu \mathrm{g}$ SR three times/week for 15 weeks and sacrificed 3 days after the last exposure. RNA was isolated from lung tissue and reverse transcribed to cDNA for real time PCR. (A) The expression of the chemokine CXCL2 and cytokines TNF $\alpha$, IL-10, IFN $\gamma$, and IL-17A was normalized to HPRT. Data is expressed as relative expression compared to WT unexposed mice \pm SD $(n=7-9$ mice per group). Significance was determined using One-way ANOVA with Tukey post hoc test $\left({ }^{*} p<0.05\right)$. (B) RNA was isolated from lung tissue and reverse transcribed to cDNA for RT-PCR. The expression of the Th2-associated chemokine CCL11/eotaxin and cytokine IL-4 was detected on an agarose gel stained with ethidium bromide. Actin expression was used as a housekeeping gene control ( $\mathrm{n}=5-7$ mice per group). 
Figure 5-3. Changes in BAL composition between 4 week and 14 week exposed mice.

WT and TLR2/ $9^{-/-}$mice were exposed to SR 3 times/week for 4 or 14 weeks and analyzed 2 days after the last exposure. BAL was performed and cells recovered from the BAL fluid were incubated with antibody to CD11b, Gr-1, CD11c, F4/80, and $\beta T c R$ and analyzed by flow cytometry. (A) The gating strategy to discriminate neutrophil $\left(\mathrm{CD} 11 \mathrm{~b}^{+}\right.$ / Gr-1 $\left.{ }^{\text {high }}\right)$, AM (CD11 $\left.\mathrm{c}^{+} / \mathrm{F} 4 / 80^{+}\right)$, macrophage $\left(\mathrm{CD} 11 \mathrm{c}^{-} / \mathrm{F} 4 / 80^{+}\right), \mathrm{DC}\left(\mathrm{CD} 11 \mathrm{c}^{+} /\right.$ $\left.\mathrm{F} 4 / 80^{-}\right)$, and $\mathrm{T}$ cell $\left(\beta \mathrm{TcR}^{+} / \mathrm{SSc}^{\text {low }}\right)$ populations present in the alveolitis. Autofluorescence ${ }^{+} \mathrm{AMs}$ were detected in the $\beta \mathrm{TcR}^{+} / \mathrm{SSc}^{\text {high }}$ gate due to spectral overlap with PE (Duan et al., 2012). (B) The percentage of neutrophils, T cells, AMs, CD11c ${ }^{+}$ DCs, and macrophages in the BAL ( $n=4-5$ mice per group). The data represent mean \pm SD and significance was determined using one-way ANOVA with Tukey post-hoc test $\left({ }^{*} p<0.05\right)$. 
A

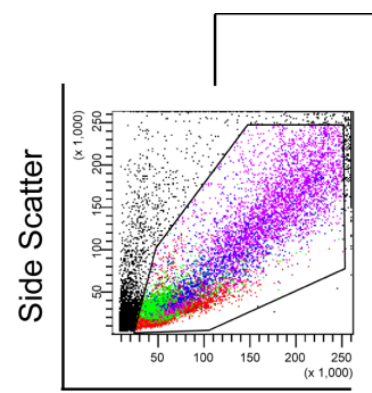

Forward Scatter

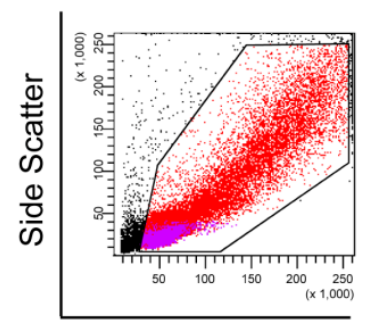

Forward Scatter

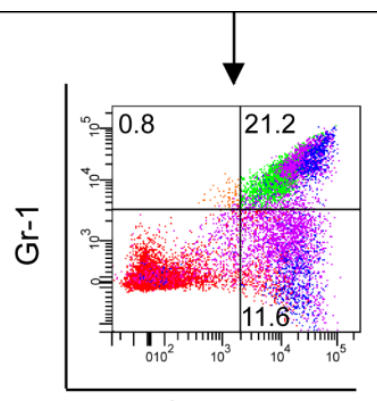

CD11b

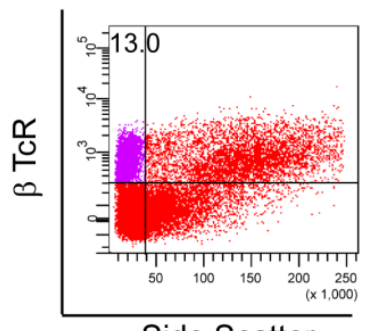

Side Scatter

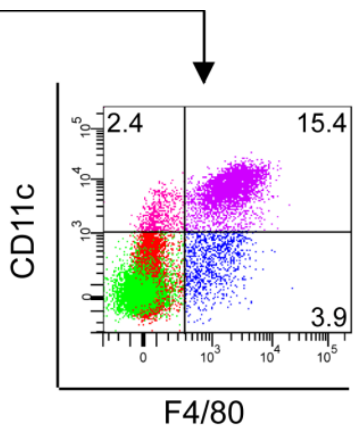

$\mathrm{F} 4 / 80$

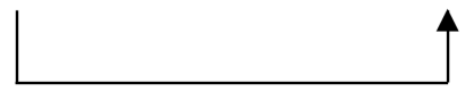

B

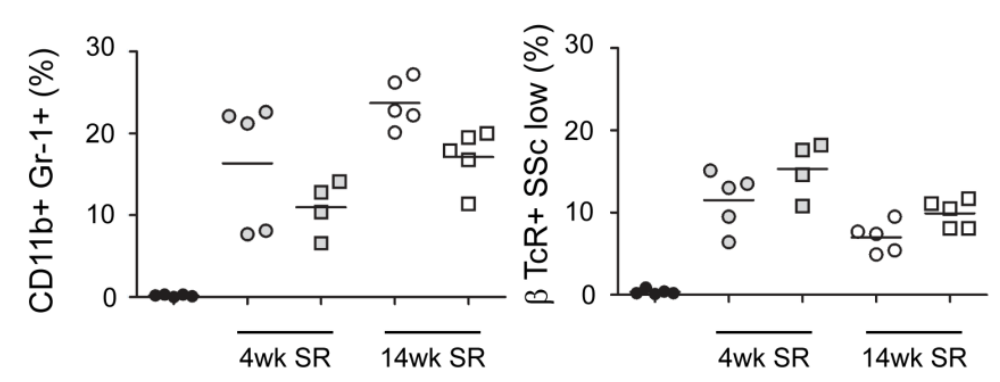

- WT Unexposed

O WT 4wk SR exp

TLR2/9 ${ }^{-/-} 4 \mathrm{wk}$ SR exp

O WT 14wk SR exp

प TLR2/9/- $14 \mathrm{wk} S R \exp$
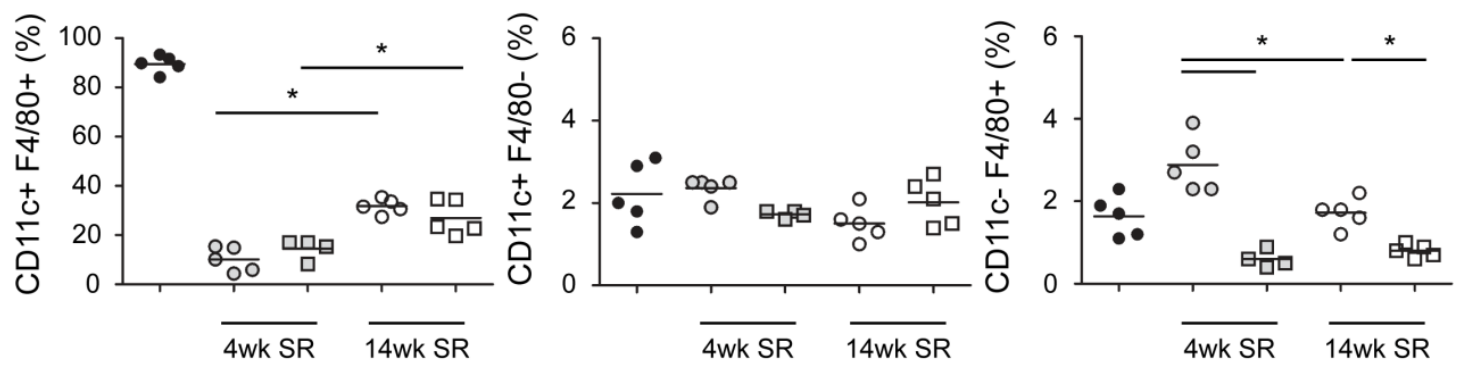
decrease in $\mathrm{CD} 11 \mathrm{c}^{-} / \mathrm{F} 4 / 80^{+}$macrophages between 4 and 14 week exposed mice. The percentages of CD11 $\mathrm{c}^{-} / \mathrm{F} 4 / 80^{+}$macrophages was significantly decreased in TLR2/9 ${ }^{-/}$ mice exposed to SR for 4 and 14 weeks compared to WT SR exposed mice. Altogether, the results suggest that neutrophils and lymphocytes are predominant cell types in the alveolitis during the granulomatous phase as well as the chronic phase of HP, but the chronic phase is associated with increased percentages of AMs.

\section{AMs Have Increased Expression of CD86 and CD206 after Long Term Exposure to SR}

During the granulomatous phase, AMs from TLR2 $/ 9^{-/}$mice had increased expression of costimulatory molecule CD80 and MHCII, markers of classically activated or type II regulatory macrophages (reviewed in Mosser, 2003; Mosser and Edwards, 2008). However, the alternative activation of macrophages is induced in the presence of IL-4, and alternatively activated macrophages are associated with the development of fibrosis (Edwards et al., 2006). The mannose receptor (CD206) is upregulated on macrophages in response to IL-4 stimulation, and is expressed on alternatively activated macrophages (Pechkovsky et al., 2010; Stein et al., 1992). Therefore, we investigated whether the development of CHP was associated with an increase in CD206 expression on AMs. In addition, we measured the expression of CD86 to determine if costimulatory molecules remain upregulated on TLR2 $/ 9^{-/-}$AMs after long term exposure to SR. Mice were intranasally exposed to SR for 4 or 14 weeks and BAL performed 2 days after the last exposure. BAL cells were stained with antibody to CD11b, CD11c, Gr-1, F4/80, CD206, and CD86 and AM expression of CD86 and CD206 determined by flow cytometry. The gating strategy to discriminate AMs is shown in Figure 5-4A. As seen previously during the granulomatous phase, TLR2 $/ 9^{-/-}$mice exposed to SR for 4 and 14 weeks had a significant reduction in the percentage of AM that express high levels of CD11b compared to WT SR exposed mice (Figure 5-4B). There was a significant increase in the geometric MFI of CD86 and CD206 on AMs from WT mice exposed to SR for 14 weeks compared to 4 weeks, but not TLR $2 / 9^{-/-}$mice (Figure 5-5). The significant increase in CD206 geometric MFI was also seen on CD11b ${ }^{\text {high }}$ AMs in mice exposed to SR for 14 weeks compared to 4 weeks (Figure 5-6), suggesting long term exposure to SR leads to increased AM activation that is dependent on TLRs 2 or 9. However, on $\mathrm{CD} 11 \mathrm{~b}$ high $\mathrm{AMs}$, TLR2 $/ 9^{-1-}$ mice had a significant increase in the percentage of cells that expressed CD86 at 4 and 14 weeks compared to WT exposed mice, suggesting TLR2 inhibits CD86 upregulation on CD11b ${ }^{\text {high }}$ AMs after short term and long term exposure to SR.

\section{TLR2 $/ 9^{-/-}$Mice Have a Significant Decrease in Activated CD4 ${ }^{+}$Th Cells after Long Term Exposure to SR}

While HP has often been associated with a lower CD4:CD8 ratio than other fibrotic lung diseases such as sarcoidosis, previous studies have suggested the 

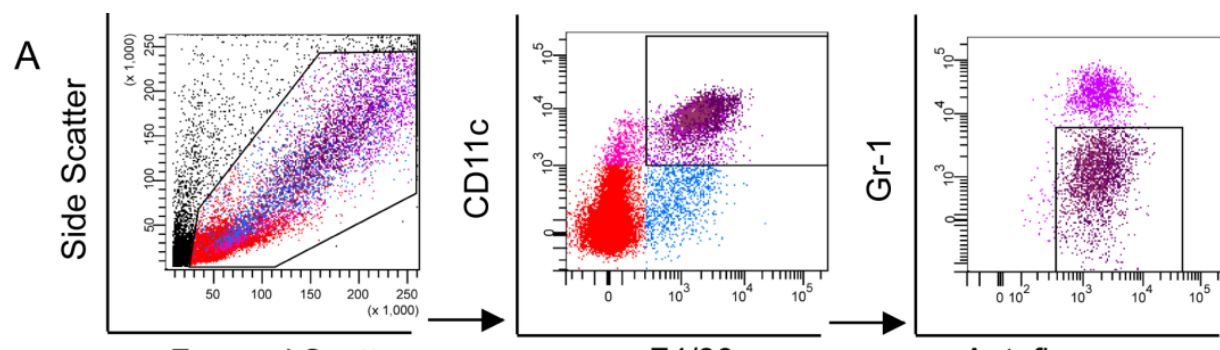

Forward Scatter

$\mathrm{F} 4 / 80$

Autofluorescence

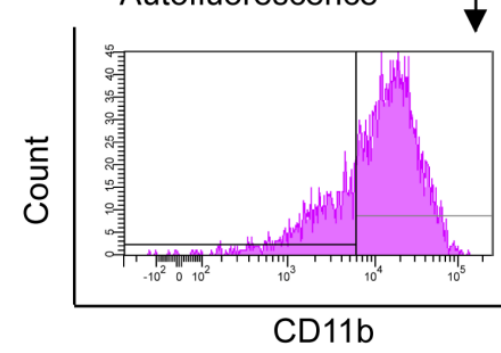

B

$\mathrm{CD} 11 \mathrm{C}+$ Autofluorescence+ F4/80+ Gr-1-

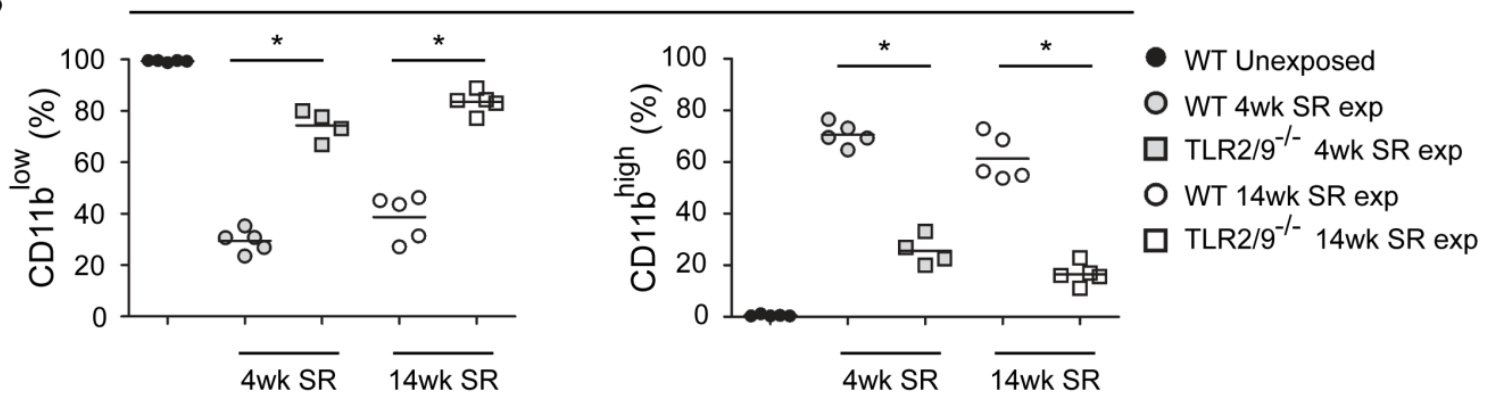

Figure 5-4. TLR2 $/ 9^{-/-}$mice have a significant decrease in CD11b $^{\text {high }}$ AMs after long term exposure to SR.

WT and TLR2 $/ 9^{-/-}$mice were exposed to SR 3 times/week for 4 or 14 weeks and analyzed 2 days after the last exposure. BAL was performed and cells recovered from the BAL fluid were incubated with antibody to CD11b, Gr-1, CD11c, F4/80, and analyzed by flow cytometry. (A) The gating strategy to discriminate CD11b $b^{\text {high }}$ and CD $11 b^{\text {low }} A M$ populations $\left(\mathrm{CD} 11 \mathrm{c}^{+} / \mathrm{F} 4 / 80^{+} /\right.$autofluorescence $\left.^{+} / \mathrm{Gr}-1^{-}\right)$present in the alveolitis. (B) The percentage of CD11 $b^{\text {low }}$ and CD $11 b^{\text {high }}$ AMs in the BAL ( $n=4-5$ mice per group). The data represent mean \pm SD and significance was determined using one-way ANOVA with Tukey post-hoc test $(* p<0.05)$. 


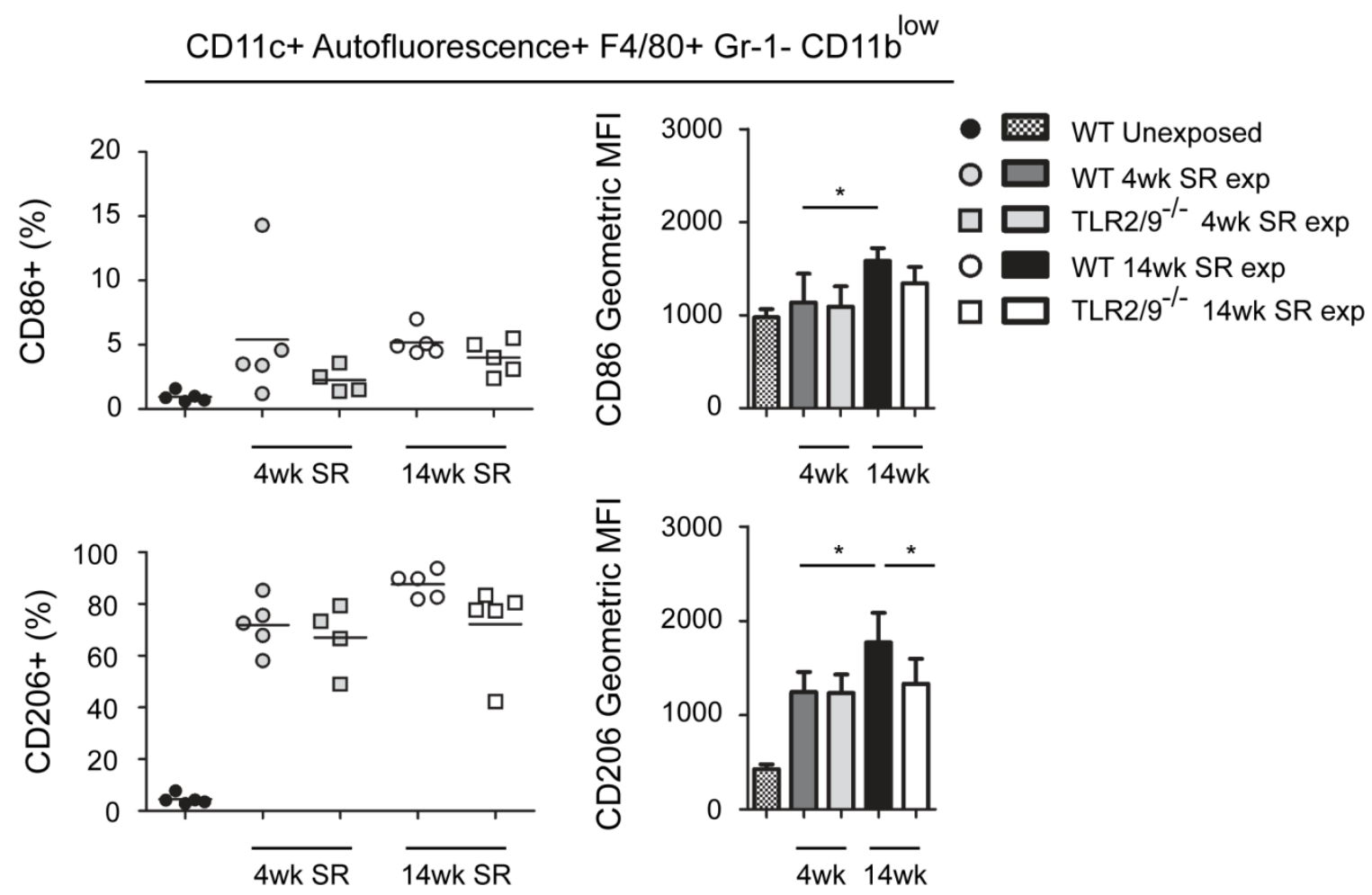

Figure 5-5. WT AMs have a significant increase in the expression of CD86 and CD206 expression after 14 weeks SR exposure.

WT and TLR2 $/ 9^{-/-}$mice were exposed to SR 3 times/week for 4 or 14 weeks and analyzed 2 days after the last exposure. BAL was performed and cells recovered from the BAL fluid were incubated with antibody to CD11b, Gr-1, CD11c, F4/80, CD86 and CD206 and analyzed by flow cytometry. The percentage of AMs (CD11c ${ }^{+} /$ autofluorescence $\left.^{+} / \mathrm{F} 4 / 80^{+} / \mathrm{Gr}^{-} 1^{-} / \mathrm{CD} 11 \mathrm{~b}^{\text {low }}\right)$ that express CD86 and CD206 and the geometric MFI was measured ( $n=4-5$ mice per group). The data represent mean $\pm \mathrm{SD}$ and significance was determined using one-way ANOVA with Tukey post-hoc test $(* p<$ $0.05)$. 


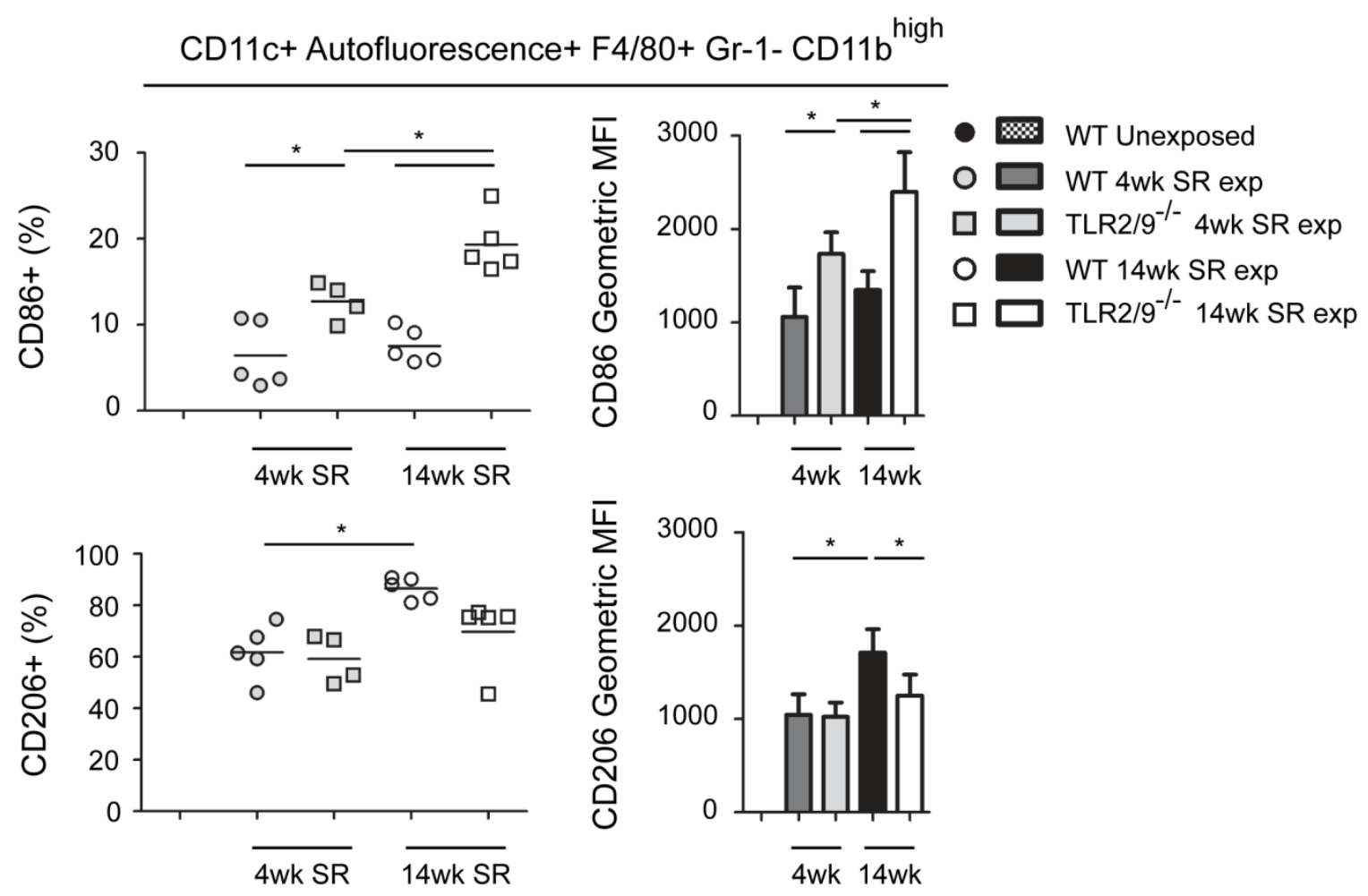

Figure 5-6. TLR2 $/ 9^{-/}$CD11b $^{\text {high }}$ AMs have increased expression of CD86 and WT $\mathrm{CD} 1 \mathrm{~b}^{\text {high }}$ AMs have increase expression of CD206 after 14 weeks exposure to SR.

WT and TLR2 $/ 9^{-/-}$mice were exposed to SR 3 times/week for 4 or 14 weeks and analyzed 2 days after the last exposure. BAL was performed and cells recovered from the BAL fluid were incubated with antibody to CD11b, Gr-1, CD11c, F4/80, CD86 and CD206 and analyzed by flow cytometry. The percentage of CD11b ${ }^{\text {high }}$ AMs (CD11 ${ }^{+} /$ autofluorescence $\left.{ }^{+} / \mathrm{F} 4 / 80^{+} / \mathrm{Gr}^{-} 1^{-} / \mathrm{CD} 11 \mathrm{~b}^{\text {high }}\right)$ that express CD86 and CD206 and the geometric MFI was measured ( $n=4-5$ mice per group). The data represent mean $\pm \mathrm{SD}$ and significance was determined using one-way ANOVA with Tukey post-hoc test $\left({ }^{*} p<\right.$ $0.05)$. 
development of CHP leads to an increase in the number of $\mathrm{CD}^{+} \mathrm{T}$ cells in the BAL and thus an increase in the CD4:CD8 ratio (Barrera et al., 2008; Welker et al., 2004). However, a recent study has shown the CD4:CD8 ratio can be variable in all stages of HP (Caillaud et al., 2012). To investigate possible increases in the CD4:CD8 ratio in mice that have been exposed long term to SR, BAL cells were collected from mice exposed to SR for 4 and 14 weeks and incubated with antibody against $\beta$ TcR, CD4, and CD8 and the percentage of $\mathrm{CD}^{+}$and $\mathrm{CD} 8^{+} \mathrm{T}$ cells was measured by flow cytometry. Unexposed mice were excluded from the analysis as T cells were not present in the BAL of unexposed mice. The gating strategy to identify $\mathrm{CD} 4^{+}$and $\mathrm{CD} 8^{+} \mathrm{T}$ cells in the BAL is depicted in Figure 5-7A. We found no differences in the percentages of $\mathrm{CD}^{+}$and $\mathrm{CD} 8^{+} \mathrm{T}$ cells between WT and TLR $2 / 9^{-/-}$mice, and no difference between mice that had been exposed to SR for 4 weeks compared to 14 weeks (Figure 5-7B). The CD4:CD8 ratio was also not significantly different between groups. The results suggest long term SR exposure does not lead to changes in the CD4:CD8 ratio in mice.

Studies during the granulomatous phase of HP found that SR induced signaling through TLR2 was important for $\mathrm{CD} 4{ }^{+}$Th cell activation, and that TLR2 $/ 9^{-/-}$mice had a significant reduction in the percentage of activated $\mathrm{CD}^{+}$Th cells in the BAL and lungs after 3 weeks exposure to SR. To determine whether TLR2 $/ 9^{-/-}$mice have a significant reduction in the percentage of activated $\mathrm{CD}^{+}$Th cells after long term SR exposure, BAL cells from WT and TLR $2 / 9^{-/-}$mice exposed to SR for 4 or 14 weeks were additionally incubated with antibody to $\mathrm{CD} 69$, and the percentage of $\mathrm{CD}^{+}$and $\mathrm{CD} 8^{+} \mathrm{T}$ cells that express CD69 was measured by flow cytometry. TLR2 $/ 9^{-/-}$mice had a significant reduction in the percentage of $\mathrm{CD}^{+} \mathrm{Th}$ cells that express $\mathrm{CD} 69^{+}$compared to WT mice after 4 and 14 weeks SR exposure (Figure 5-7C). There was no significant difference in the percentage of $\mathrm{CD} 69^{+} \mathrm{CD} 8^{+} \mathrm{T}$ cells between WT and TLR2/9 $/ 9^{-/}$mice. The results suggest SR induced signaling through TLR2 is also important for $\mathrm{CD}^{+}$Th cell activation during chronic HP.

\section{Mice with Long Term Exposure to SR Have Increased Expression of IFN $\gamma$ and IL-17A in the Lung but not IL-4}

During the granulomatous phase, the expression of TNF $\alpha$ was significantly reduced in the lungs of TLR2 $2^{-/}$and TLR $2 / 9^{-/-}$mice. To investigate the expression of $\mathrm{TNF} \alpha$ and as well as the anti-inflammatory cytokine IL-10 during chronic HP, mice were exposed to SR 3 times a week for 15 weeks and the lung tissue harvested for RNA isolation and qRT-PCR. The relative expression of the inflammatory cytokine TNF $\alpha$ and the anti-inflammatory cytokine IL-10 was increased in mice exposed to SR for 15 weeks over unexposed mice (Figure 5-2A); however, there was no significant difference between WT and TLR2 $/ 9^{---}$SR exposed mice. The results suggest the loss of TLR2 does not impact TNF $\alpha$ expression during chronic HP.

Previous studies of CHP in the pigeon breeder's disease HP model have suggested the expression of Th2-associated cytokines, IL-4 and IL-13, is associated with increased 
A

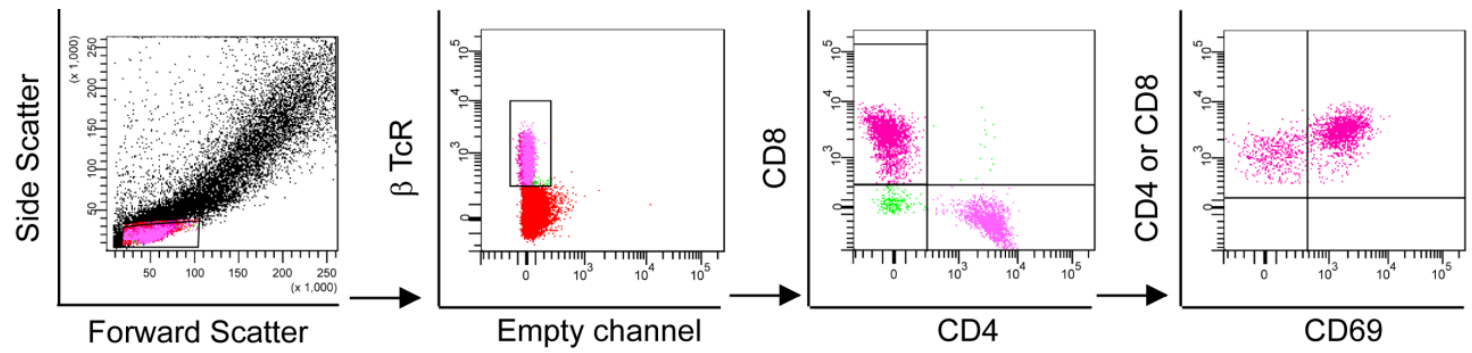

B

$\mathrm{CD} 45+\beta \mathrm{TcR}+$

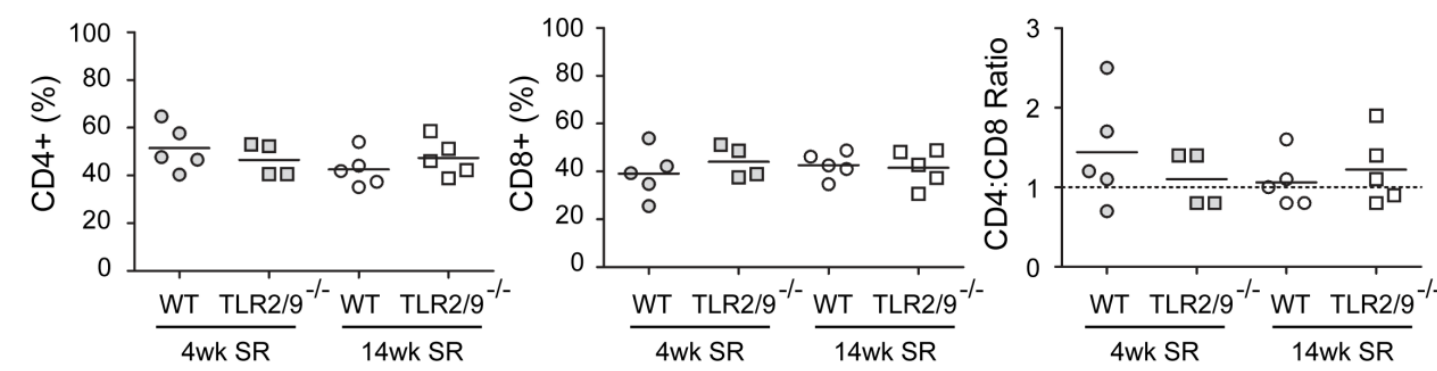

C

$\mathrm{CD} 45+\beta \mathrm{TcR}+\mathrm{CD} 4+$

$\mathrm{CD} 45+\beta \mathrm{TCR}+\mathrm{CD} 8+$

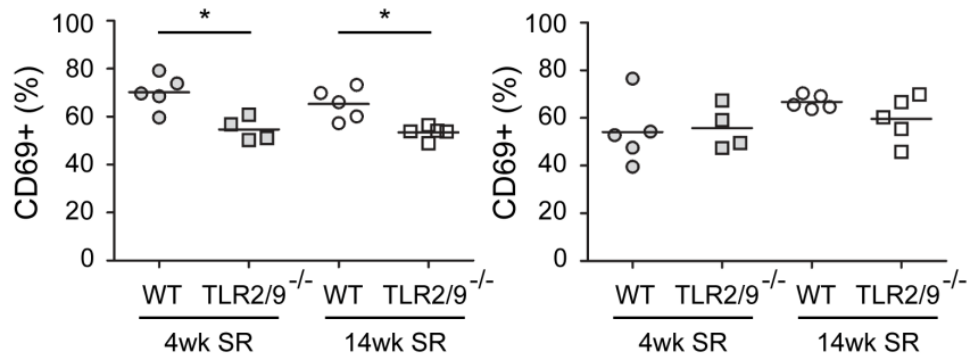

Figure 5-7. $\mathrm{CD4}^{+}$and $\mathrm{CD8}^{+} \mathrm{T}$ cells in mice exposed to SR 4 and 14 weeks.

WT and TLR2 $/ 9^{-/-}$mice were exposed to SR 3 times/week for 4 or 14 weeks and analyzed 2 days after the last exposure. BAL was performed and cells recovered from the BAL fluid were incubated with antibody to $\beta$ TcR, CD4, CD8, and CD69 and analyzed by flow cytometry. (A) The gating strategy to discriminate $\mathrm{CD}^{+}$Th cells $\left(\mathrm{CD} 45^{+} / \beta \mathrm{TcR}^{+} /\right.$ $\left.\mathrm{CD}^{+}\right)$and $\mathrm{CD} 8^{+} \mathrm{T}$ cells $\left(\mathrm{CD} 45^{+} / \beta \mathrm{TcR}^{+} / \mathrm{CD}^{+}\right)$that express $\mathrm{CD} 69$ in the lymphocyte gated alveolitis population. (B) The percentage of $\mathrm{CD}^{+}{ }^{+} \mathrm{Th}$ cells and $\mathrm{CD} 8^{+} \mathrm{T}$ cells in the BAL and the CD4:CD8 ratio. (C) The percentage of $\mathrm{CD}^{+}{ }^{\mathrm{T}}$ Th cells or $\mathrm{CD} 8{ }^{+} \mathrm{T}$ cells that express $\mathrm{CD} 69$ ( $n=3$ - 4 mice per group). The data represent mean $\pm \mathrm{SD}$ and significance was determined using one-way ANOVA with Tukey post-hoc test $\left({ }^{*} p<0.05\right)$. 
lung fibrosis in the Th2-biased mouse strain A/J (Mitaka et al., 2011). However, in the SR model of HP, IL-17 and a Th17 response is associated with increased fibrosis (Joshi et al., 2009; Simonian et al., 2009). Therefore, cytokine expression was examined in mice with SR induced CHP to quantify the relative expression of cytokines associated with Th1, Th2, and Th17 responses, IFN $\gamma$, IL-4, and IL-17A respectively. RNA was isolated from whole lungs for reverse transcription and real time RT-PCR. The relative expression of IFN $\gamma$ and IL-17A was significantly increased in 15 week exposed mice compared to unexposed mice (Figure 5-2A). During the granulomatous phase, TLR $2 / 9^{-/-}$mice had a significant decrease in IL-17 expression and the Th17 response during the granulomatous phase. Surprisingly, TLR $2 / 9^{-/}$mice exposed long term to SR had a significant increase in IL-17A expression compared to WT SR exposed mice. The expression of IL-4 was not detected at this time point (Figure 5-2B), and IL-22, a Th17 associated cytokine that has been shown to protect against lung fibrosis in a mouse model of HP (Simonian et al., 2010), was also not detected at this time point. Therefore, the results show that mice exposed to SR express inflammatory cytokines IFN $\gamma$, IL-17A, and TNF $\alpha$ as well as the anti-inflammatory cytokine IL-10, but do not express IL-4 or IL-22. Altogether, the results suggest after long term exposure to SR, mice have increased expression of Th1 and Th17 associated cytokines IFN $\gamma$ and IL-17, but not the Th2 associated cytokine IL-4.

\section{CD4 $^{+}$Th Cells from Mice with Long Term Exposure to SR Express IL-17A}

To investigate whether the polarization of $\mathrm{T}$ cells in the lung switches to a Th2 response in mice after long term exposure to SR, WT and TLR2 $/ 9^{-/-}$mice were exposed to SR for 14 weeks and dispersed lung cells were stimulated ex vivo with PMA/ionomycin in the presence of brefeldin A and incubated with antibody to CD45, F4/80, $\beta$ TcR, CD4, IL-17, and IFN $\gamma$ and the percentage of Th17 and Th2 cells in the lung was examined by intracellular cytokine staining. The gating strategy to discriminate $\mathrm{CD}^{+}$Th cells in a population of dispersed lung cells is shown in Figure 5-8A. The results show the percentage of IL-17A and IL-4 expressing $\mathrm{CD}^{+}{ }^{+} \mathrm{Th}$ cells in Figure 5-8B. A small percentage of IL-17A expressing $\mathrm{CD}^{+}$Th cells was detected in WT SR exposed mice, and TLR $2 / 9^{-/-}$mice had a significant increase in the percentage of IL-17A expressing $\mathrm{CD}^{+}$Th cells compared to unexposed mice (Figure 5-8C). There was no significant increase in the percentage of Th cells that express IL-4 in WT or TLR2 $/ 9^{-/-}$SR exposed mice at this time point. Therefore, after long term SR exposure Th cells are polarized to a Th17 response, and do not switch to a Th2 response.

To investigate the contribution of other T cells populations in IL-17A and IL-4 expression after long term exposure to SR, dispersed lung cells were gated on $\mathrm{CD}^{-}$ $\beta \mathrm{TcR}^{+} \mathrm{T}$ cells (Figure 5-9A), and the percentage of IL-17 and IL-4 expressing $\mathrm{CD}^{-}{ }^{-} \mathrm{T}$ cells is shown in (Figure 5-9B). There was no significant increase in the percentage of $\mathrm{CD}^{-}$Th cells expressing IL-17A or IL-4 after 14 weeks SR exposure (Figure 5-9C). Recent studies have suggested neutrophils and macrophages also contribute to IL-17 production and the fibrotic response in HP (Hasan et al., 2013). To determine if macrophages are a greater contributor to IL-17A or IL-4 production after long term 


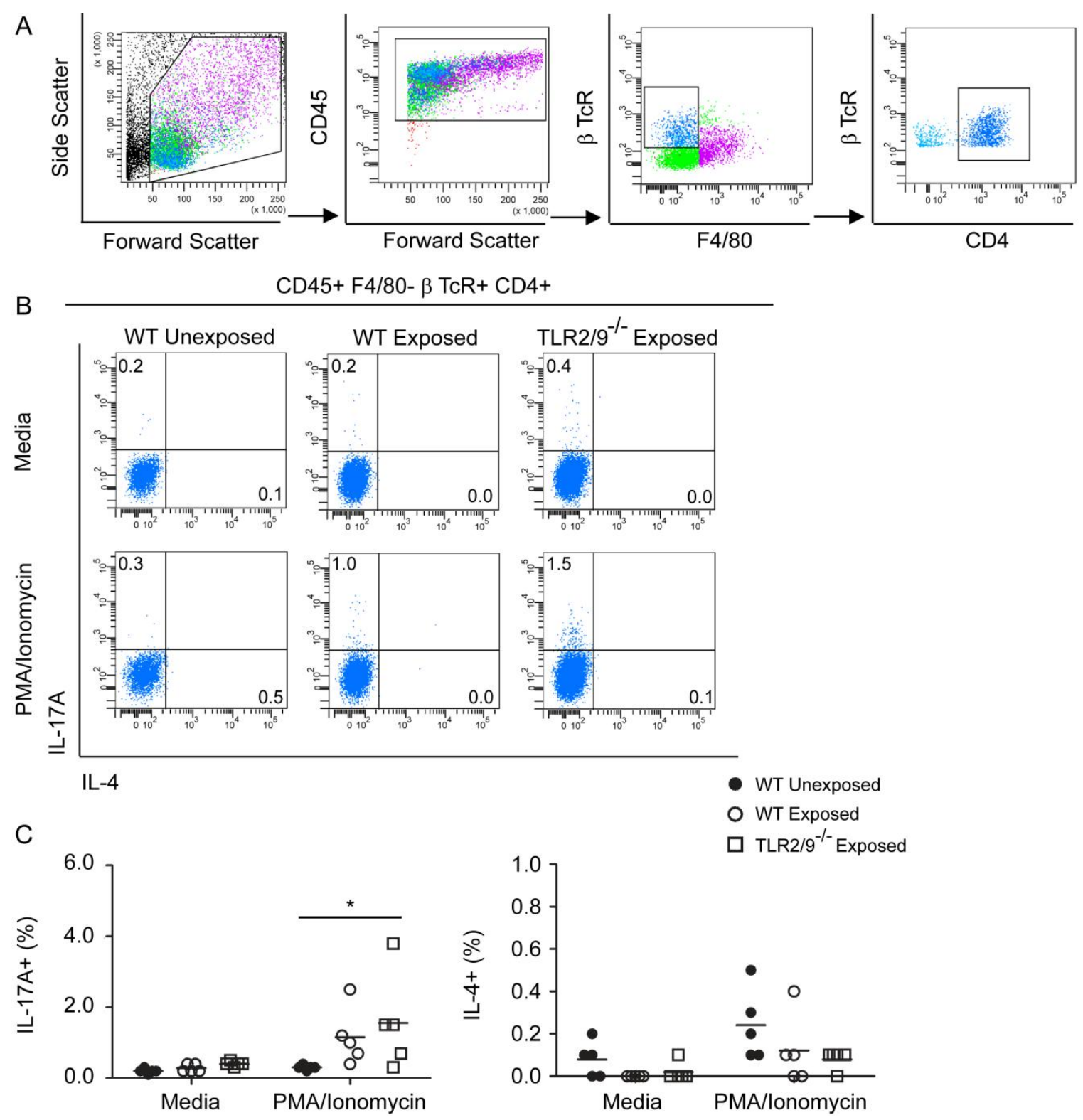

Figure 5-8. CD4 ${ }^{+}$Th cells in TLR2/9 ${ }^{-1-}$ mice express IL-17 but not IL-4 after long term exposure to $\mathrm{SR}$.

WT and TLR2 $/ 9^{--}$mice were exposed to SR 3 times/week for 14 weeks and analyzed 2 days after the last exposure. Lung cells were digested and stimulated with PMA/Ionomycin or media alone in the presence of brefeldin A then incubated with antibodies for CD45, $\beta T c R, C D 4, F 4 / 80$, IL-17A, and IL-4 for intracellular cytokine staining. (A) Gating strategy for the identification of $\mathrm{CD}^{+}$Th cells $\left(\mathrm{CD} 45^{+} / \mathrm{F} 4 / 80^{-} /\right.$ $\beta \mathrm{TcR}^{+} / \mathrm{CD}^{+}$) in dispersed lung cells. (B) Representative dot plots of media or PMA/ionomycin stimulated $\mathrm{CD}^{+}$Th cells expressing IL-17A or IL-4. (C) The percentage of $\mathrm{CD}^{+}$Th cells expressing IL-17 or IL-4. ( $\mathrm{n}=5$ mice / group.) Significance was determined using two-way ANOVA with Bonferroni post-hoc test $(* p<0.05$ compared to WT SR exposed mice). 
A

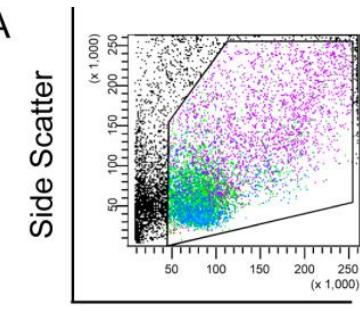

Forward Scatter
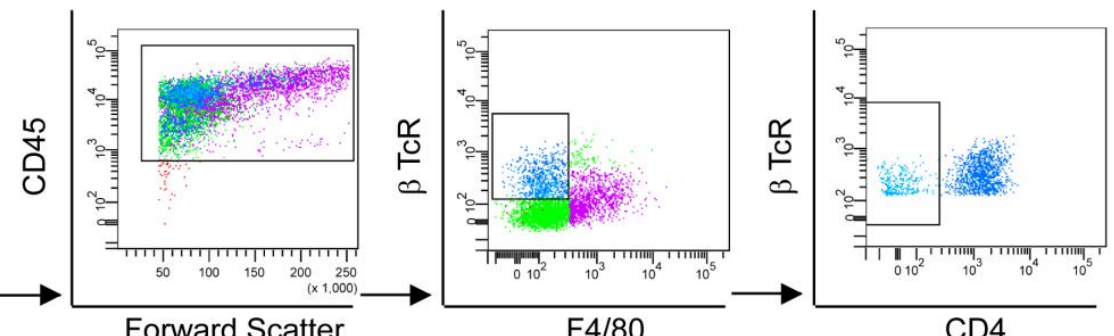

$\mathrm{B}$

$\mathrm{CD} 45+\mathrm{F} 4 / 80-\beta \mathrm{TCR}+\mathrm{CD} 4-$
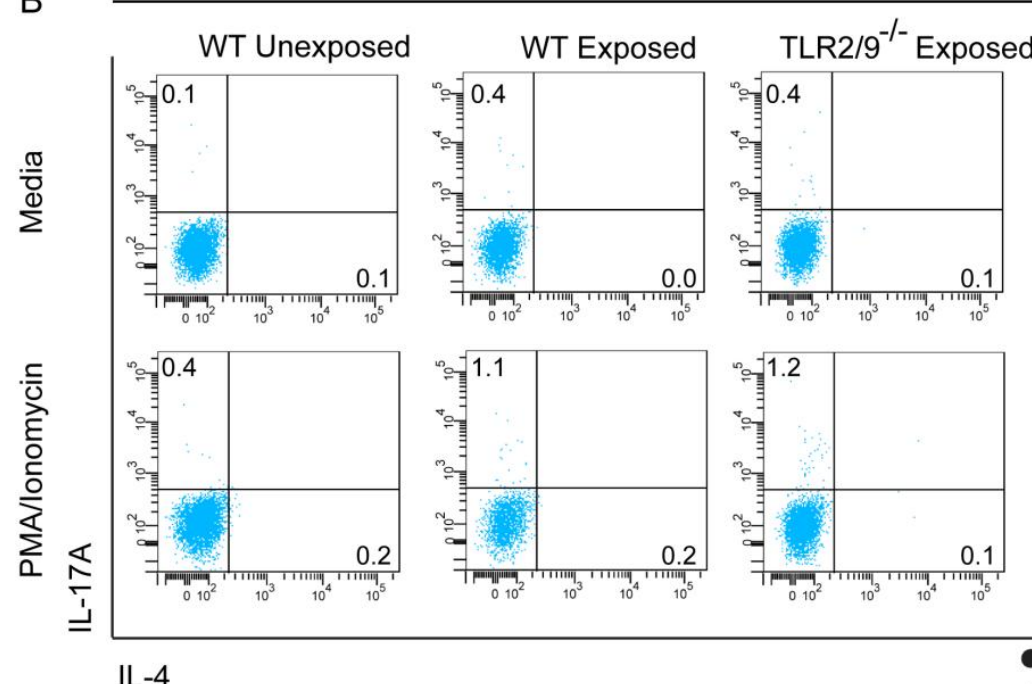

C
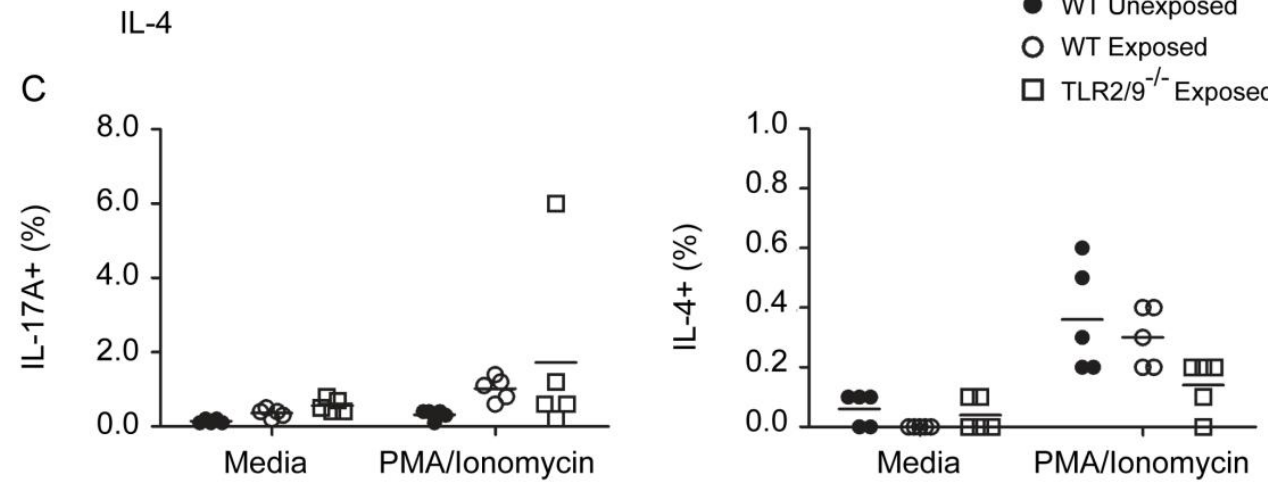

Figure 5-9. $\mathrm{CD}^{-} \mathrm{T}$ cells do not have a significant increase in IL-17 or IL-4 expression after long term exposure to $\mathrm{SR}$.

WT and TLR2 $/ 9^{-/-}$mice were exposed to SR 3 times/week for 14 weeks and analyzed 2 days after the last exposure. Lung cells were digested and stimulated with PMA/Ionomycin or media alone in the presence of brefeldin A then incubated with antibodies for CD45, $\beta \mathrm{TcR}, \mathrm{CD} 4, \mathrm{~F} 4 / 80$, IL-17A, and IL-4 for intracellular cytokine staining. (A) Gating strategy for the identification of $\mathrm{CD}^{-}$Th cells $\left(\mathrm{CD} 45^{+} / \mathrm{F} 4 / 80^{-}\right.$/ $\beta \mathrm{TcR}^{+} / \mathrm{CD}^{-}$) in dispersed lung cells. (B) Representative dot plots of media or PMA/ionomycin stimulated $\mathrm{CD}^{-}$Th cells expressing IL-17A or IL-4. (C) The percentage of $\mathrm{CD}^{-}$Th cells expressing IL-17 or IL-4. ( $\mathrm{n}=5$ mice / group.) Significance was determined using two-way ANOVA with Bonferroni post-hoc test $(* p<0.05$ compared to WT SR exposed mice). 
exposure to SR, dispersed lung cells stimulated with PMA/ionomycin were gated on macrophages (Figure 5-10A), and the percentage of macrophages that express IL-17 or IL-4 are shown in Figure 5-10B. A small percentage of IL-17 expressing macrophages were detected in SR exposed mice, and TLR2 $/ 9^{-/-}$mice had a significant increase in the percentage of IL-17 expressing macrophages over unexposed mice (Figure 5-10C). IL-4 expression was not detected in macrophages at this time point. Altogether, the results suggest Th17 cells and IL-17A expressing macrophages are present in the lungs of mice with CHP, and long term exposure to SR does not lead to a switch to a Th2 response.

\section{Mice with CHP Have Decreased Barrier Function and Lung Fibrosis}

Lung pathology was examined by assessing barrier function and lung fibrosis in mice exposed long term to SR. Total protein and IgM was measured in the BALF in mice exposed to SR for 4 or 14 weeks as a measure of protein leak across the lung epithelium. WT and TLR $2 / 9^{-1-}$ mice exposed to SR for 14 weeks had a significant increase in total protein compared to unexposed mice (Figure 5-11). Interestingly, total IgM was significantly decreased after 14 weeks exposure to SR compared to 4 weeks exposure. To measure lung fibrosis that develops following 14 weeks of SR exposure, lungs from WT and TLR2 $/ 9^{-/-}$SR exposed and control mice were stained with Masson's trichrome. The slides were digitized and blue pixels quantified in the lung sections with a positive pixel algorithm. Representative images show increased collagen deposition in the alveolar walls in mice exposed to 14 weeks SR compared to normal lung architecture unexposed

mice (Figure 5-12A-C). The positive pixel analysis showed WT mice exposed to SR for 14 weeks had a significantly greater number of positive and strong positive pixels per analysis area compared to unexposed mice and TLR2/9 $/ 9^{-1-}$ mice (Figure 5-12D). When only strong positive pixels were considered, WT mice again had a significant increase compared to unexposed and TLR $2 / 9^{-/-}$mice. This suggests TLR $2 / 9^{-/-}$may be partially protected from the development of fibrosis after long term SR exposure. Altogether, the results suggest mice exposed to SR for 14 weeks have loss of barrier function and lung fibrosis compared to unexposed mice.

\section{Mice with CHP Have Reduced Static Lung Compliance and Total Lung Capacity}

Edema as a result of epithelial barrier disruption as well as lung fibrosis can contribute to reduced lung compliance. To assess whether mice exposed to SR develop reduced lung compliance and reduced total lung capacity similar to patients with $\mathrm{CHP}$, mice exposed to SR for 15 weeks were placed on a flexiVent ${ }^{\mathrm{TM}}$ ventilator to assess lung function through a snap shot perturbation and a pressure volume curve. During a snap shot perturbation, the air volume delivered to the lungs is oscillated in a sinusoidal wave form (Figure 5-13A). Pressure and volume data is collected and fit to the single compartment model of the lung to calculate lung compliance, elastance, and resistance (reviewed in Bates and Lutchen, 2005). To generate a pressure volume loop, the air volume delivered to the mouse is increased in a step-wise fashion up to total lung 
Figure 5-10. Macrophages contribute to IL-17 expression in TLR2/9 ${ }^{-/-}$mice after long term exposure to SR.

WT and TLR2 $/ 9^{-/-}$mice were exposed to SR 3 times/week for 14 weeks and analyzed 2 days after the last exposure. Lung cells were digested and stimulated with PMA/Ionomycin or media alone in the presence of brefeldin $\mathrm{A}$ then incubated with antibodies for CD45, $\beta T c R, C D 4, F 4 / 80$, IL-17A, and IL-4 for intracellular cytokine staining. (A) Gating strategy for the identification of macrophages (CD45 $/ \mathrm{F} 4 / 80^{+} /$ $\beta T c R^{-}$) in dispersed lung cells. (B) Representative dot plots of media or PMA/ionomycin stimulated macrophages expressing IL-17A or IL-4. (C) The percentage of macrophages expressing IL-17 or IL-4. ( $\mathrm{n}=5$ mice / group.) Significance was determined using twoway ANOVA with Bonferroni post-hoc test $(* p<0.05$ compared to WT SR exposed mice). 
A
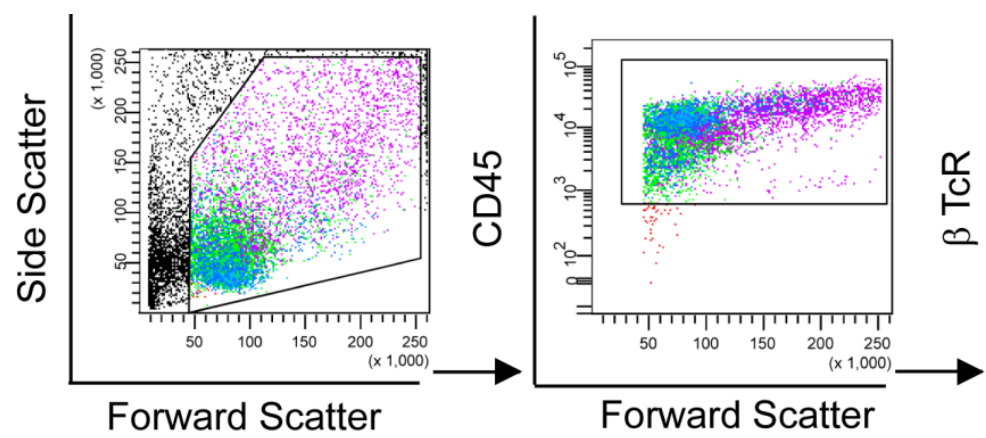

Forward Scatter

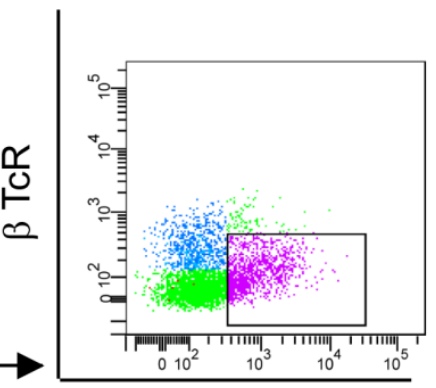

$\mathrm{F} 4 / 80$

B

CD45+ F4/80+ $\beta$ TcR-
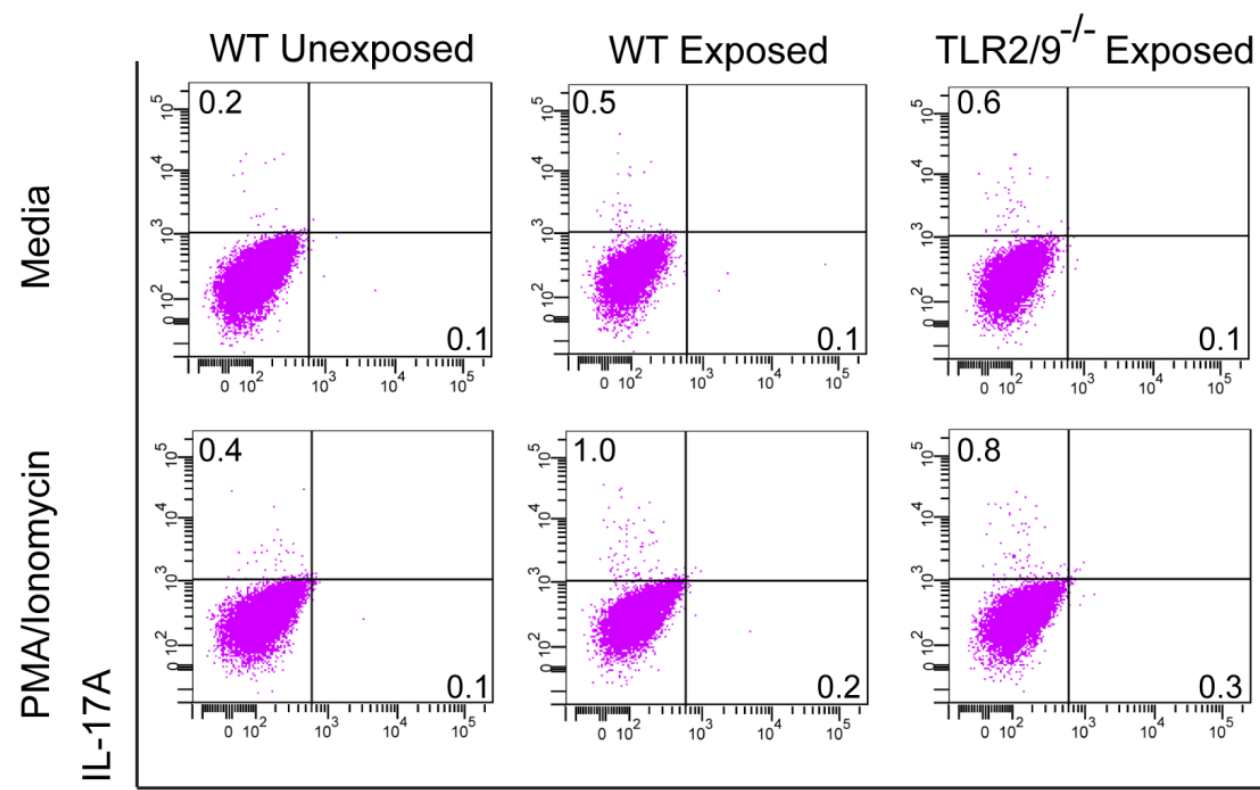

IL-4

- WT Unexposed

O WT Exposed
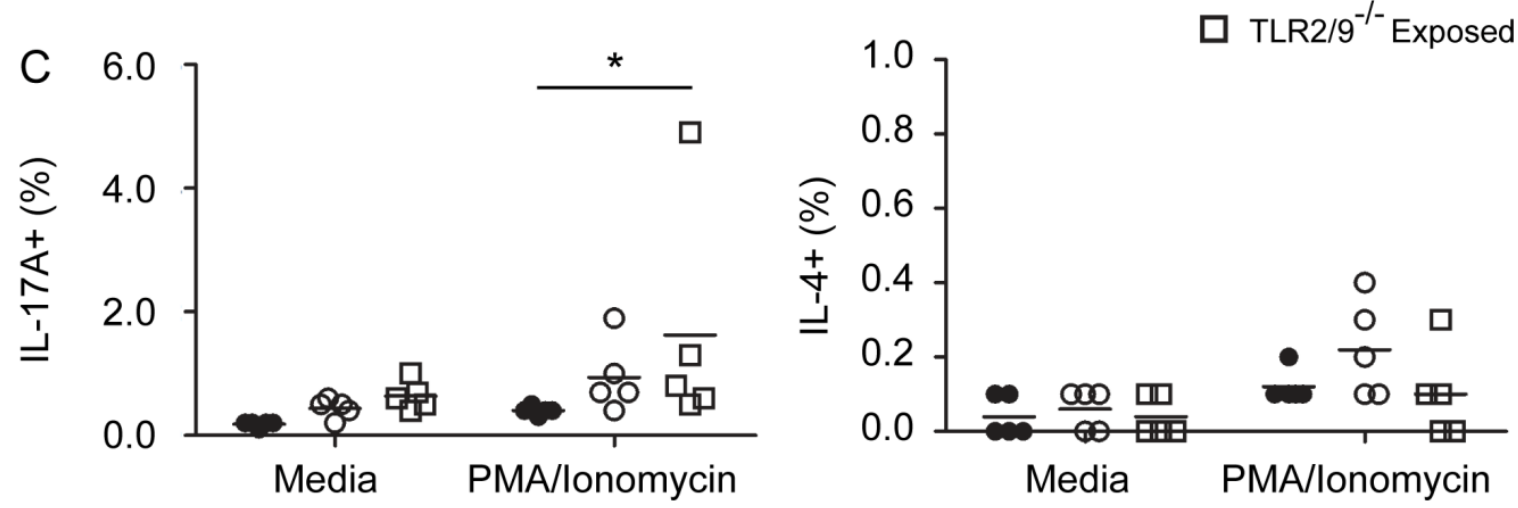

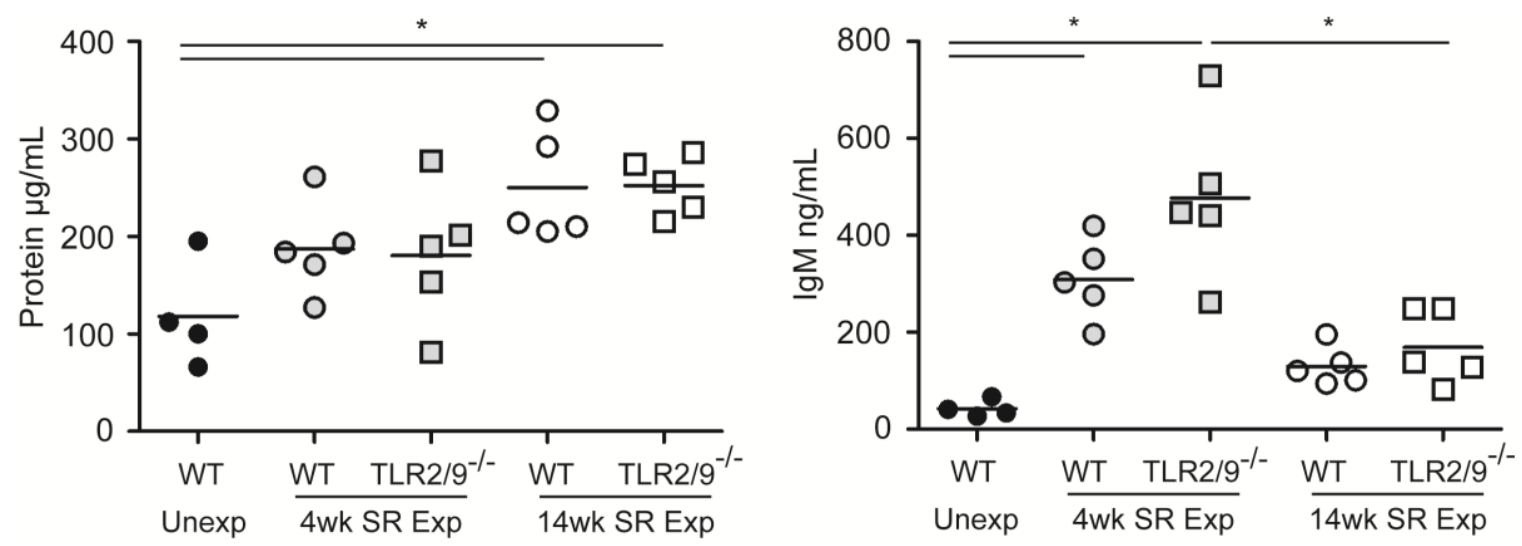

Figure 5-11. Evidence of plasma protein leakage across the lung epithelium in the BALF of mice exposed to SR for 4 and 14 weeks.

WT and TLR2 $/ 9^{-/-}$mice were exposed to SR 3 times/week for 4 or 14 weeks and sacrificed 2 days after the last exposure. The cell-free BAL fluid was analyzed for total protein by Bio-rad protein assay and IgM by ELISA to detect protein leakage across the epithelium as an indicator of disrupted barrier disruption. Data represent the mean $\pm \mathrm{SD}$ ( $\mathrm{n}=4-5$ per group). Significance was calculated using one-way ANOVA with Tukey post-hoc test $(* p<0.05$ compared to WT SR exposed mice). 

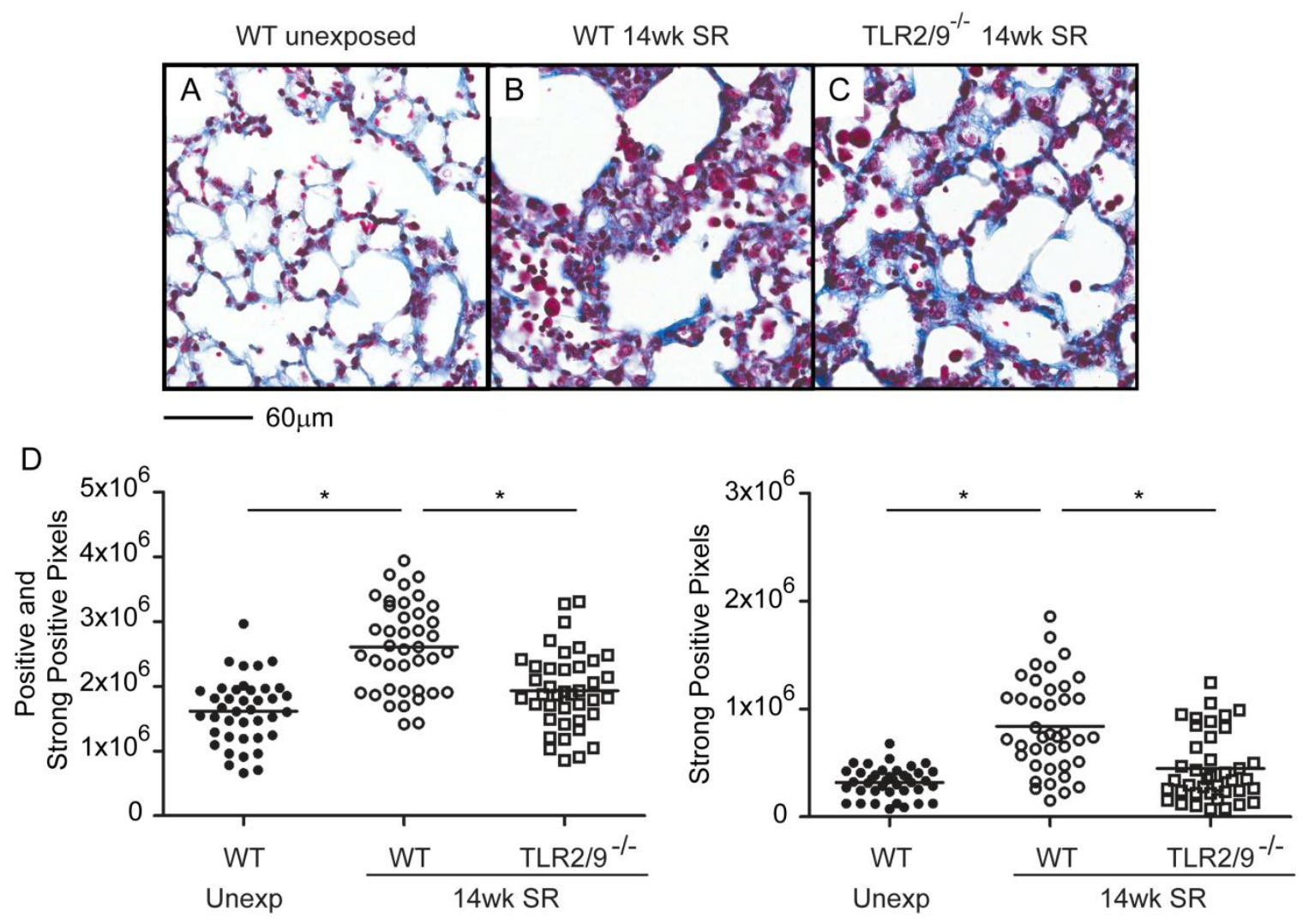

Figure 5-12. WT mice have a significant increase in lung fibrosis.

WT and TLR2 $/ 9^{-/-}$mice were exposed to SR for 14 weeks and lung tissue was harvested for histological analysis. Lung slides were stained with Masson's trichrome then digitized for image analysis. Representative images from (A) WT unexposed, (B) WT 14 week SR exposed, and (C) TLR2/9 $9^{-/} 14$ week SR exposed mice were captured at 40x. (D) The Aperio positive pixel algorithm was used to quantify blue collagen pixels in $8 \times 1 \mathrm{~mm}^{2}$ areas / section. The total number of positive and strong positive pixels was counted. $(\mathrm{n}=$ 5 mice / group). The data represent mean \pm SD and significance was determined using one-way ANOVA with Tukey post-hoc test $(* p<0.05)$. 


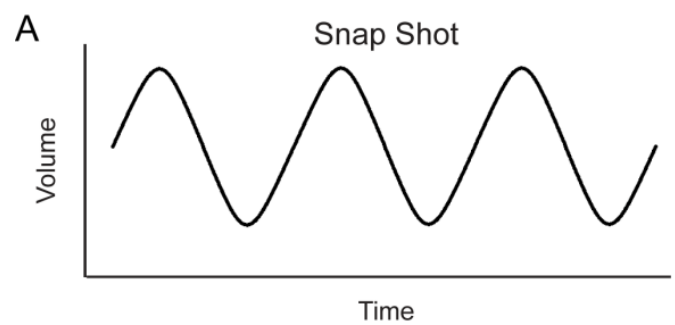

Single compartment model of the lung

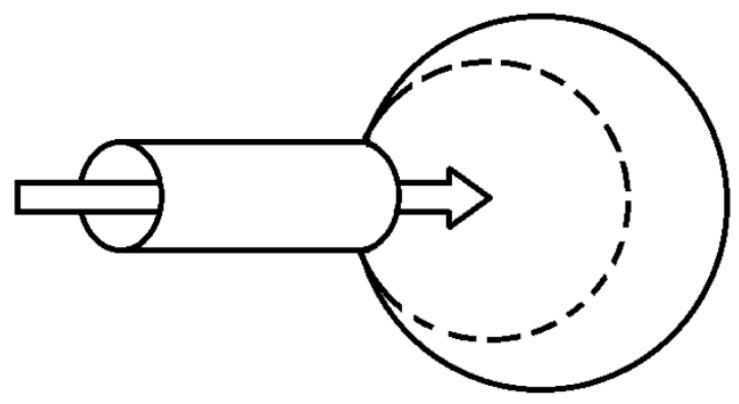

Pressure $=$ Elastance ${ }^{*}$ Volume + Resitance ${ }^{*}$ Flow + P0

Elastance $=\Delta$ Pressure $/ \Delta$ Volume

Compliance $=1 /$ Elastance $=\Delta$ Volume $/ \Delta$ Pressure

Resistance $=\Delta$ Pressure $/$ Flow

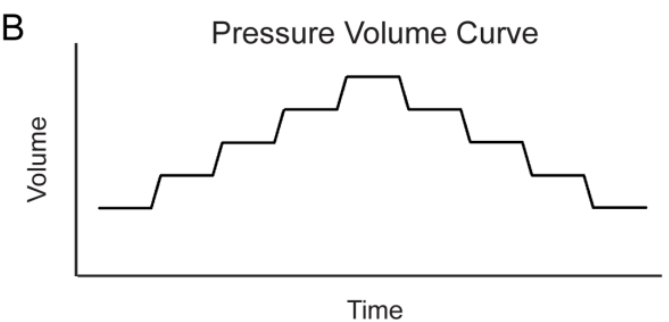

Pressure Volume Curve

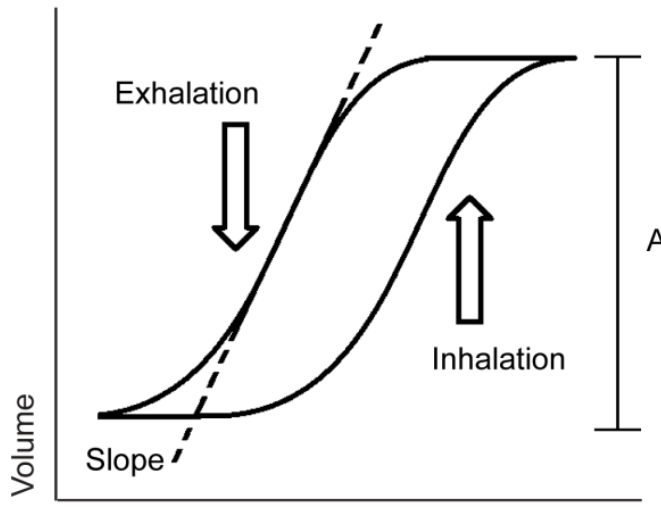

Pressure

Total Volume $=\mathrm{A}-\mathrm{Be}-\mathrm{kPlateau}$ Pressure

Static Compliance $=$ Slope of curve at given volume Static Elastance $=1 /$ Static Compliance

$A=$ Upper bounds estimate of the difference between total lung capacity and zero volume

\section{Figure 5-13. FlexiVent ${ }^{\mathrm{TM}}$ snap shot and pressure volume curve perturbations.}

The flexiVent ${ }^{\mathrm{TM}}$ mechanical ventilator is a computer controlled piston pump capable of manipulating volume and pressure during ventilation to collect lung function data. (A) In a snap shot perturbation, the air volume delivered to the lungs is oscillated in a sinusoidal waveform. Pressure and volume data are collected during the maneuver and fit to the single compartment model of the lung consisting of a rigid tube connected to a flexible balloon to calculate elastance, compliance, and resistance of the lung. (B) To generate a pressure volume curve, the volume of air delivered to the lungs is increased in stepwise increments up to the total lung capacity and decreased to functional residual lung capacity. Pressure and volume data is collected when the air is not flowing (static) and fit to the Salazar-Knowles equation to calculate static compliance, static elastance, and total lung capacity. 
capacity, and returned to functional residual capacity (Figure 5-13B). Pressure and volume data is collected when air is not moving or "static", then the Salazar-Knowles equation is fit to the data (Salazar and Knowles, 1964). The static compliance is the slope, and the total lung capacity is the upper bounds limit of the Salazar-Knowles equation.

During the snap shot perturbation, WT and TLR $2 / 9^{-/-}$mice exposed long term to SR had a significant reduction in lung compliance compared to unexposed mice (Figure 5-14A). Elastance is the inverse of compliance, and therefore, WT and TLR2 $/ 9^{-/-}$mice had a significant increase in lung elastance compared to unexposed mice. Lung resistance was also significantly increased in WT and TLR2 $/ 9^{-/-}$mice compared to unexposed mice. The pressure volume curve also revealed WT and TLR2 $/ 9^{-1-}$ mice exposed to SR for 15 weeks had a significant reduction in static compliance, and a significant increase in static elastance compared to unexposed mice (Figure 5-14B). The total lung capacity was significantly decreased in WT SR exposed mice compared to unexposed mice, but not TLR2 $/ 9^{-/-}$mice. These results suggest WT and TLR $2 / 9^{-/-}$mice exposed to SR for 15 weeks have reduced lung compliance consistent with the development of restrictive lung disease.

\section{Discussion}

The results of our studies suggest mice exposed long term to SR do not switch to a Th2 immune response. Th2 cells were not detected by intracellular cytokine staining in the lungs of mice exposed to SR for 14 weeks. Recruitment of eosinophils to sites of inflammation is associated with Th2 immune responses. Analysis of the BAL cells reveals only a small percentage of eosinophils are recruited to the lungs of WT and TLR2 $/ 9^{-/-}$mice after 15 weeks SR exposure, and little to no IL-4 expression, one of the signature cytokines of a Th2 response, is detected in the lungs by RT-PCR at this time point. The majority of $C D 11 b^{\text {low }}$ and $C D 11 b^{\text {high }}$ AMs in 4 and 14 week exposed mice expressed CD206, and CD206 upregulation on macrophages is associated with alternative activation and exposure to IL-4. However, previous studies have shown TGF $\beta$ and GM-CSF also upregulate CD206 on macrophages in vitro (Porcheray et al., 2005), suggesting the upregulation of CD206 on AMs is not dependent on IL-4 in SR exposed mice.

IL-17 expression and a Th17 immune response after 3-4 week exposure to SR is associated with increased disease severity and fibrosis in HP (Hasan et al., 2013; Joshi et al., 2009; Simonian et al., 2009). The results of this study show long term exposure to SR is also associated with a Th17 response. Th17 cells are detected in the lungs of WT and TLR2 $/ 9^{-/-}$mice exposed to SR for 14 weeks by intracellular flow cytometry. Results from real time RT-PCR show lung tissue from mice exposed to SR for 15 weeks had a strong induction of IL-17 expression over unexposed mice, and was significantly increased in TLR2 $/ 9^{-1-}$ mice over WT exposed mice. IL-17A is expressed by Th17 cells, but can also be expressed by other cell types such as $\gamma \delta \mathrm{T}$ cells and NK cells. However, 

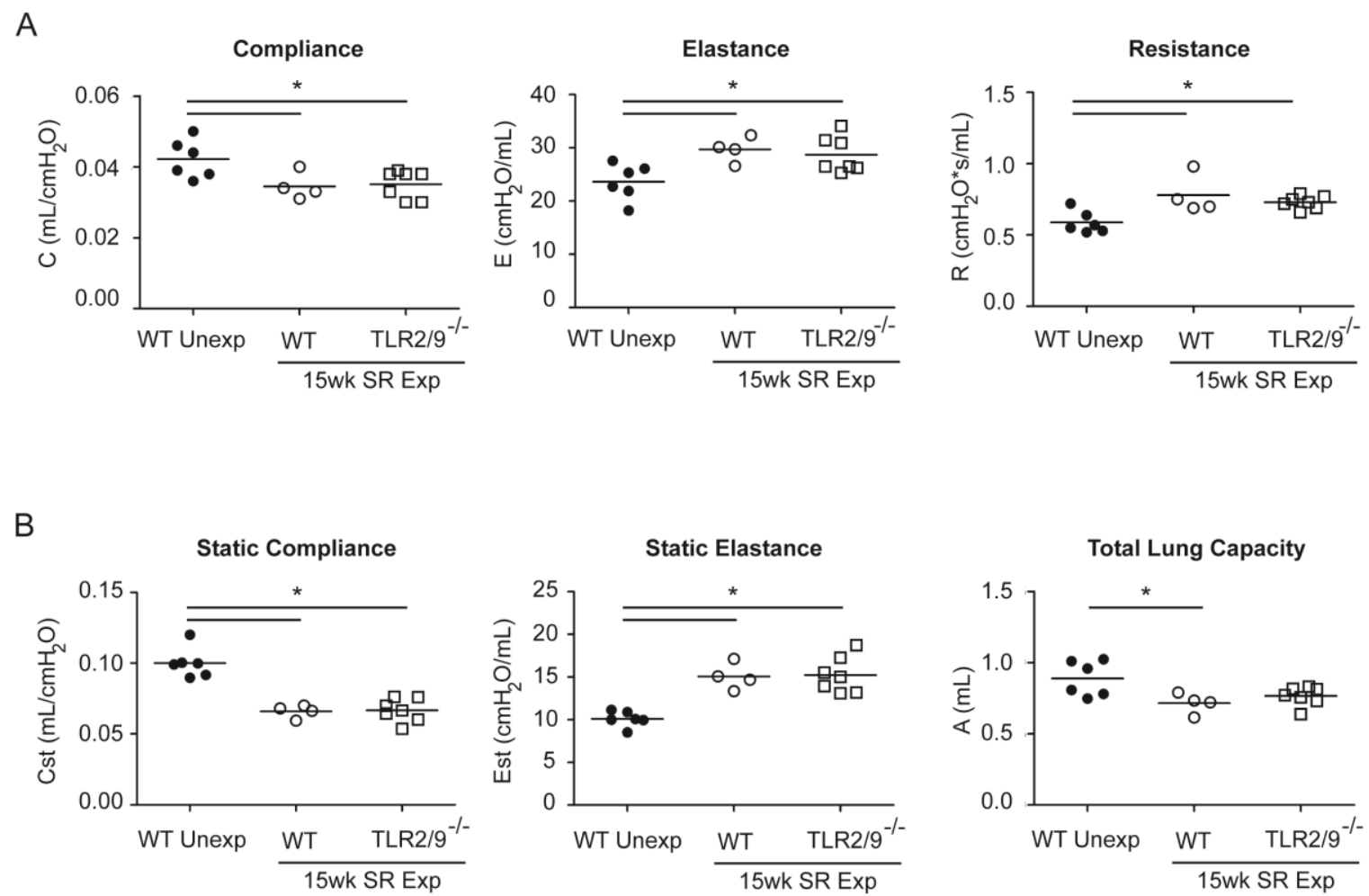

\section{Figure 5-14. WT and TLR2/9 ${ }^{-1-}$ mice have a significant decrease in lung compliance.}

WT and TLR2 $/ 9^{-/-}$mice were exposed to SR for 15 weeks and analyzed 3 days after the last exposure. Mice were placed on a flexiVent ${ }^{\mathrm{TM}}$ mechanical ventilator for lung function analysis ( $n=4-7$ mice per group). (A) A snap shot perturbation was performed to measure compliance, elastance, and resistance. (B) A pressure volume curve was performed to measure static compliance, static elastance, and total lung capacity. Each point represents the average value of at least 2 measurements for each mouse. The data represent mean $\pm \mathrm{SD}$ and significance was determined using one-way ANOVA with Tukey post-hoc test $(* p<0.05)$. 
recent studies in mice exposed to $\mathrm{SR}$ for 3 weeks showed that neutrophils and macrophages were the main cell type that expressed IL-17A and that neutrophil depletion decreased lung collagen (Hasan et al., 2013). This is in agreement with studies that have shown a positive correlation between the percentage of neutrophils in the lung tissue and the percentage of lung fibrosis in patients with HP (Pardo et al., 2000). Intercellular cytokine staining revealed a small percentage of macrophages expressed IL-17A in WT and TLR2 $/ 9^{-/-}$mice exposed to SR for 15 weeks. Differential counting of BAL cells and analysis of BAL cell populations by flow cytometry revealed an increase in the percentage of neutrophils and lymphocytes suggesting both cell types contribute to lung pathology at this time point.

However, the relationship between inflammation and fibrosis is not so clear cut. Inflammation mediated by the innate and adaptive immune system precedes fibrosis not only in the lung, but in multiple organ systems (reviewed in Wick et al., 2013), but the mechanisms that tip the balance between fibrosis and normal wound repair are still the subject of ongoing research. One hypothesis put forward by Dr. Robert Streiter suggests lung fibrosis develops when the integrity of the basement membrane is lost, which prevents the reestablishment of normal lung architecture, and progresses in the presence of chronic inflammation and persistent antigen (Strieter, 2008). In HP, neutrophils may damage basement membrane integrity through the secretion of collagenase- 2 and gelatinase B (Pardo et al., 2000).

Granuloma formation is dependent on $\mathrm{CD}^{+} \mathrm{T}$ cells and the cytokines TNF $\alpha$ and IFN $\gamma$ (Denis et al., 1991; Gudmundsson and Hunninghake, 1997). Mice exposed to SR for 15 weeks have increased expression of TNF $\alpha$ and IFN $\gamma$ compared to unexposed mice and peri-vascular and peri-bronchial granulomas can be observed in lung sections. Activated $\mathrm{CD}^{+}{ }^{+} \mathrm{T}$ cells are also significantly increased in the BALF of mice exposed to 14 weeks SR but significantly reduced in TLR $2 / 9^{-/-}$mice as seen previously during the granulomatous phase. Additional experiments are necessary to determine if $\mathrm{T}$ cells or non-T cells are the source of IFN $\gamma$ and TNF $\alpha$ expression at this time point.

Increased lung fibrosis leads to reduced lung compliance and restrictive lung disease. Other mouse models of fibrotic lung diseases have shown reduced lung compliance (Vanoirbeek et al., 2010; Wang et al., 2011), however, this is the first study to measure lung function in a mouse model of HP. WT and TLR2 $/ 9^{-/-}$mice exposed to SR for 15 weeks have a significant decrease in static lung compliance compared to unexposed mice. Lung barrier disruption leading to edema may contribute to reduced lung compliance as WT and TLR2 $/ 9^{-/-}$mice had a significant increase in total protein in the BALF compared to unexposed mice. The decrease in lung compliance was associated with reduced total lung capacity and increased fibrosis in WT mice exposed to SR for 14 weeks suggesting WT mice developed restrictive lung disease. Fibrosis was present in TLR $2 / 9^{-/-}$mice, but positive pixel analysis revealed a significant reduction collagen deposition in the lungs of TLR $2 / 9^{-1-}$ mice compared to WT SR exposed mice. In addition, the total lung capacity in $\mathrm{TLR} 2 / 9^{-/}$mice was not significantly reduced compared to unexposed mice. Together, the results suggest TLR2/9 ${ }^{-1-}$ mice may be 
partially protected from the development of lung fibrosis, but are not protected from the development of CHP.

Overall, the results show mice with long term exposure to SR develop lung fibrosis and reduced lung compliance. We find no evidence of a switch to a Th2 immune response, but rather detect Th17 cells and increased expression of IL-17A. In addition, TLR2 $/ 9^{-/-}$mice have a greater increase in the percentage of Th17 cells in the lung and expression of IL-17. TLR2/9 $9^{-/-}$mice may have a partial reduction in the development of lung fibrosis compared to WT SR exposed mice, but are not protected from the development of interstitial lung disease as they have similar reductions in lung compliance as WT mice. 


\section{CHAPTER 6. FUTURE DIRECTIONS}

Treatment options for HP are limited, and include antigen avoidance and corticosteroids. The results of my study suggest TLRs 2 and 9 play an important role in the pathogenesis of HP. Mice deficient in TLRs 2 and 9 have significant reductions in neutrophil recruitment, inflammatory cytokine production, macrophage and lymphocyte activation, and the Th17 response. In addition, TLR $2 / 9^{-/}$mice were partially protected from the development of lung fibrosis. This suggests TLRs 2 and 9 are attractive targets for pharmacological inhibition of HP. TLR antagonists are currently in development to modulate immune responses in inflammatory disorders and autoimmune diseases. Drugs currently in development block TLR binding or disrupt TLR signaling, and many are entering clinical trials.

Among the TLR antagonist currently being developed, OPN-305 is a humanized version of the mouse-anti human TLR2-blocking antibody OPN-301, which has shown promise in the prevention of cardiac and kidney reperfusion injury, and inhibition of cytokine release by ex vivo stimulated synovial cells in rheumatoid arthritis (Arslan et al., 2012; Arslan et al., 2010; Farrar et al., 2012; Ultaigh et al., 2011). OPN-305 has recently finished phase I clinical trial and shows promise for further development. AN3485 is another compound that has been shown to antagonize TLR2, TLR3, TLR4, and TLR5 signaling; and mice treated with the compound had reduced TNF $\alpha$, IL-1 $\beta$, and IL-6 production in mouse models of sepsis, contact-dermatitis, and collagen-induced arthritis (Dong et al., 2013). Synthetic oligonucleotides are also being developed to inhibit TLR9 and have been shown to be effective in inhibiting immune responses in mouse models of rheumatoid arthritis, lupus, multiple sclerosis, and psoriasis (Barrat et al., 2007; Ho et al., 2003; Jiang et al., 2013; Zeuner et al., 2002). Further experiments to investigate the therapeutic potential of TLR2 and TLR9 antagonists in SR exposed mice are warranted.

Perhaps more importantly, during the course of my study, our lab has developed a mouse model of SR induced CHP. By establishing a time point where mice exposed to SR develop lung fibrosis and decreased lung compliance, we can study other immune modulatory compounds that may inhibit the progression to CHP. The CD200R is an inhibitory receptor expressed on myeloid cells and lymphocytes, which binds CD200 expressed on immune and non-immune cells (Rijkers et al., 2008). CD200 induced signaling through CD200R has been shown to be important for controlling inflammation as mice deficient in CD200 exhibit exacerbated immune responses during viral infection or autoimmune disease (Hoek et al., 2000; Snelgrove et al., 2008). Treatment with a CD200 fusion protein (CD200-Fc) has shown promise as an inhibitor of innate immune inflammation in mouse models of collagen-induced arthritis and multiple sclerosis (Liu et al., 2010; Simelyte et al., 2008). Experiments to test the efficacy of CD200-Fc treatment in SR exposed mice are planned. For further discussion on future directions see Appendix. 


\section{LIST OF REFERENCES}

Abdelsamed, H.A., Desai, M., Nance, S.C., and Fitzpatrick, E.A. (2011). T-bet controls severity of hypersensitivity pneumonitis. J Inflamm (Lond) 8,15 .

Abraham, E., Freitas, A.A., and Coutinho, A.A. (1990). Purification and characterization of intraparenchymal lung lymphocytes. The Journal of Immunology 144, 21172122.

Acharya, M., Mukhopadhyay, S., Paidassi, H., Jamil, T., Chow, C., Kissler, S., Stuart, L.M., Hynes, R.O., and Lacy-Hulbert, A. (2010). alphav Integrin expression by DCs is required for Th17 cell differentiation and development of experimental autoimmune encephalomyelitis in mice. J Clin Invest 120, 4445-4452.

Arslan, F., Houtgraaf, J.H., Keogh, B., Kazemi, K., de Jong, R., McCormack, W.J., O'Neill, L.A., McGuirk, P., Timmers, L., Smeets, M.B., et al. (2012). Treatment with OPN-305, a humanized anti-Toll-Like receptor-2 antibody, reduces myocardial ischemia/reperfusion injury in pigs. Circulation. Cardiovascular interventions 5, 279-287.

Arslan, F., Smeets, M.B., O'Neill, L.A., Keogh, B., McGuirk, P., Timmers, L., Tersteeg, C., Hoefer, I.E., Doevendans, P.A., Pasterkamp, G., and de Kleijn, D.P. (2010). Myocardial ischemia/reperfusion injury is mediated by leukocytic toll-like receptor- 2 and reduced by systemic administration of a novel anti-toll-like receptor-2 antibody. Circulation 121, 80-90.

Bafica, A., Santiago, H.C., Goldszmid, R., Ropert, C., Gazzinelli, R.T., and Sher, A. (2006). Cutting edge: TLR9 and TLR2 signaling together account for MyD88dependent control of parasitemia in Trypanosoma cruzi infection. J Immunol 177, 3515-3519.

Bafica, A., Scanga, C.A., Feng, C.G., Leifer, C., Cheever, A., and Sher, A. (2005). TLR9 regulates Th1 responses and cooperates with TLR2 in mediating optimal resistance to Mycobacterium tuberculosis. J Exp Med 202, 1715-1724.

Barrat, F.J., Meeker, T., Chan, J.H., Guiducci, C., and Coffman, R.L. (2007). Treatment of lupus-prone mice with a dual inhibitor of TLR7 and TLR9 leads to reduction of autoantibody production and amelioration of disease symptoms. Eur J Immunol $37,3582-3586$.

Barrera, L., Mendoza, F., Zuniga, J., Estrada, A., Zamora, A.C., Melendro, E.I., Ramirez, R., Pardo, A., and Selman, M. (2008). Functional diversity of T-cell subpopulations in subacute and chronic hypersensitivity pneumonitis. Am J Respir Crit Care Med 177, 44-55. 
Bashir, N., Kounsar, F., Mukhopadhyay, S., and Hasnain, S.E. (2010). Mycobacterium tuberculosis conserved hypothetical protein $\mathrm{rRv} 2626 \mathrm{c}$ modulates macrophage effector functions. Immunology 130, 34-45.

Bates, J.H.T., and Lutchen, K.R. (2005). The interface between measurement and modeling of peripheral lung mechanics. Respiratory physiology \& neurobiology $148,153-164$.

Bettelli, E., Carrier, Y., Gao, W., Korn, T., Strom, T.B., Oukka, M., Weiner, H.L., and Kuchroo, V.K. (2006). Reciprocal developmental pathways for the generation of pathogenic effector TH17 and regulatory T cells. Nature 441, 235-238.

Bhan, U., Newstead, M.J., Zeng, X., Ballinger, M.N., Standiford, L.R., and Standiford, T.J. (2011). Stachybotrys chartarum-induced hypersensitivity pneumonitis is TLR9 dependent. Am J Pathol 179, 2779-2787.

Bogdan, C., Röllinghoff, M., and Diefenbach, A. (2000). Reactive oxygen and reactive nitrogen intermediates in innate and specific immunity. Current Opinion in Immunology 12, 64-76.

Bourgeois, C., Majer, O., Frohner, I.E., Lesiak-Markowicz, I., Hildering, K.S., Glaser, W., Stockinger, S., Decker, T., Akira, S., Muller, M., and Kuchler, K. (2011). Conventional Dendritic Cells Mount a Type I IFN Response against Candida spp. Requiring Novel Phagosomal TLR7-Mediated IFN- Signaling. The Journal of Immunology 186, 3104-3112.

Butler, N.S., Monick, M.M., Yarovinsky, T.O., Powers, L.S., and Hunninghake, G.W. (2002). Altered IL-4 mRNA stability correlates with Th1 and Th2 bias and susceptibility to hypersensitivity pneumonitis in two inbred strains of mice. $\mathrm{J}$ Immunol 169, 3700-3709.

Caillaud, D.M., Vergnon, J.M., Madroszyk, A., Melloni, B.M., Murris, M., and Dalphin, J.C. (2012). Bronchoalveolar lavage in hypersensitivity pneumonitis: a series of 139 patients. Inflammation \& allergy drug targets 11, 15-19.

Carvalho, N.B., Oliveira, F.S., Duraes, F.V., de Almeida, L.A., Florido, M., Prata, L.O., Caliari, M.V., Appelberg, R., and Oliveira, S.C. (2011). Toll-like receptor 9 is required for full host resistance to Mycobacterium avium infection but plays no role in induction of Th1 responses. Infect Immun 79, 1638-1646.

Charo, I.F., and Ransohoff, R.M. (2006). The many roles of chemokines and chemokine receptors in inflammation. The New England journal of medicine 354, 610-621.

Cherfils-Vicini, J., Platonova, S., Gillard, M., Laurans, L., Validire, P., Caliandro, R., Magdeleinat, P., Mami-Chouaib, F., Dieu-Nosjean, M.C., Fridman, W.H., et al. (2010). Triggering of TLR7 and TLR8 expressed by human lung cancer cells induces cell survival and chemoresistance. J Clin Invest 120, 1285-1297. 
Costabel, U., Bonella, F., and Guzman, J. (2012). Chronic hypersensitivity pneumonitis. Clinics in chest medicine 33, 151-163.

Daito, H., Kikuchi, T., Sakakibara, T., Gomi, K., Damayanti, T., Zaini, J., Tode, N., Kanehira, M., Koyama, S., Fujimura, S., et al. (2011). Mycobacterial hypersensitivity pneumonitis requires TLR9-MyD88 in lung CD11b+ CD11c+ cells. The European respiratory journal 38, 688-701.

Denis, M. (1992). Interleukin-6 in mouse hypersensitivity pneumonitis: changes in lung free cells following depletion of endogenous IL-6 or direct administration of IL-6. J Leukoc Biol 52, 197-201.

Denis, M., Cormier, Y., Fournier, M., Tardif, J., and Laviolette, M. (1991). Tumor necrosis factor plays an essential role in determining hypersensitivity pneumonitis in a mouse model. Am J Respir Cell Mol Biol 5, 477-483.

Denis, M., Cormier, Y., and Laviolette, M. (1992). Murine hypersensitivity pneumonitis: a study of cellular infiltrates and cytokine production and its modulation by cyclosporin A. Am J Respir Cell Mol Biol 6, 68-74.

Didierlaurent, A., Goulding, J., Patel, S., Snelgrove, R., Low, L., Bebien, M., Lawrence, T., van Rijt, L.S., Lambrecht, B.N., Sirard, J.-C., and Hussell, T. (2008). Sustained desensitization to bacterial Toll-like receptor ligands after resolutionof respiratory influenza infection. J. Exp. Med. 205, 323-329.

Dong, C., Sexton, H., Gertrudes, A., Akama, T., Martin, S., Virtucio, C., Chen, C.W., Fan, X., Wu, A., Bu, W., et al. (2013). Inhibition of Toll-like receptor-mediated inflammation in vitro and in vivo by a novel benzoxaborole. The Journal of pharmacology and experimental therapeutics 344, 436-446.

Dong, L., Wang, S., Chen, M., Li, H., and Bi, W. (2008). The activation of macrophage and upregulation of CD40 costimulatory molecule in lipopolysaccharide-induced acute lung injury. Journal of biomedicine \& biotechnology $2008,852571$.

Duan, M., Li, W.C., Vlahos, R., Maxwell, M.J., Anderson, G.P., and Hibbs, M.L. (2012). Distinct macrophage subpopulations characterize acute infection and chronic inflammatory lung disease. J Immunol 189, 946-955.

Edwards, J.P., Zhang, X., Frauwirth, K.A., and Mosser, D.M. (2006). Biochemical and functional characterization of three activated macrophage populations. J Leukoc Biol 80, 1298-1307.

Embley, T.M., O'Donnell, A.G., Rostron, J., and Goodfellow, M. (1988). Chemotaxonomy of Wall Type IV Actinomycetes Which Lack Mycolic Acids. Journal of general microbiology 134, 953-960. 
Erkinjuntti-Pekkanen, R., Rytkonen, H., Kokkarinen, J.I., Tukiainen, H.O., Partanen, K., and Terho, E.O. (1998). Long-term risk of emphysema in patients with farmer's lung and matched control farmers. Am J Respir Crit Care Med 158, 662-665.

Ewald, S.E., Lee, B.L., Lau, L., Wickliffe, K.E., Shi, G.P., Chapman, H.A., and Barton, G.M. (2008). The ectodomain of Toll-like receptor 9 is cleaved to generate a functional receptor. Nature 456, 658-662.

Fantini, M.C., Becker, C., Monteleone, G., Pallone, F., Galle, P.R., and Neurath, M.F. (2004). Cutting Edge: TGF- $\beta$ Induces a Regulatory Phenotype in CD4+CD25- T Cells through Foxp3 Induction and Down-Regulation of Smad7. The Journal of Immunology 172, 5149-5153.

Farber, J.M. (1997). Mig and IP-10: CXC chemokines that target lymphocytes. J Leukoc Biol 61, 246-257.

Farrar, C.A., Keogh, B., McCormack, W., O'Shaughnessy, A., Parker, A., Reilly, M., and Sacks, S.H. (2012). Inhibition of TLR2 promotes graft function in a murine model of renal transplant ischemia-reperfusion injury. FASEB journal : official publication of the Federation of American Societies for Experimental Biology 26, 799-807.

Fernandez Perez, E.R., Swigris, J.J., Forssen, A.V., Tourin, O., Solomon, J.J., Huie, T.J., Olson, A.L., and Brown, K.K. (2013). Identifying an Inciting Antigen is Associated with Improved Survival in Patients with Chronic Hypersensitivity Pneumonitis. Chest.

Fong, D.J., Hogaboam, C.M., Matsuno, Y., Akira, S., Uematsu, S., and Joshi, A.D. (2010). Toll-like receptor 6 drives interleukin-17A expression during experimental hypersensitivity pneumonitis. Immunology 130, 125-136.

Fournier, E., Tonnel, A.B., Gosset, P., Wallaert, B., Ameisen, J.C., and Voisin, C. (1985). Early neutrophil alveolitis after antigen inhalation in hypersensitivity pneumonitis. Chest $88,563-566$.

Fu, S., Zhang, N., Yopp, A.C., Chen, D., Mao, M., Chen, D., Zhang, H., Ding, Y., and Bromberg, J.S. (2004). TGF- $\beta$ Induces Foxp3 + T-Regulatory Cells from CD4 + CD25 - Precursors. American Journal of Transplantation 4, 1614-1627.

Gasse, P., Riteau, N., Charron, S., Girre, S., Fick, L., Petrilli, V., Tschopp, J., Lagente, V., Quesniaux, V.F., Ryffel, B., and Couillin, I. (2009). Uric acid is a danger signal activating NALP3 inflammasome in lung injury inflammation and fibrosis. Am J Respir Crit Care Med 179, 903-913.

Gharaee-Kermani, M., Nozaki, Y., Hatano, K., and Phan, S.H. (2001). Lung interleukin-4 gene expression in a murine model of bleomycin-induced pulmonary fibrosis. Cytokine 15, 138-147. 
Gibbons, M.A., MacKinnon, A.C., Ramachandran, P., Dhaliwal, K., Duffin, R., Phythian-Adams, A.T., van Rooijen, N., Haslett, C., Howie, S.E., Simpson, A.J., et al. (2011). Ly6Chi Monocytes Direct Alternatively Activated Profibrotic Macrophage Regulation of Lung Fibrosis. American Journal of Respiratory and Critical Care Medicine 184, 569-581.

Girard, M., Israel-Assayag, E., and Cormier, Y. (2009). Mature CD11c(+) cells are enhanced in hypersensitivity pneumonitis. The European respiratory journal 34, 749-756.

Girard, M., Israel-Assayag, E., and Cormier, Y. (2011). Impaired function of regulatory T-cells in hypersensitivity pneumonitis. The European respiratory journal 37, 632-639.

Gudmundsson, G., and Hunninghake, G.W. (1997). Interferon-gamma is necessary for the expression of hypersensitivity pneumonitis. J Clin Invest 99, 2386-2390.

Gudmundsson, G., Monick, M.M., and Hunninghake, G.W. (1998). IL-12 modulates expression of hypersensitivity pneumonitis. J Immunol 161, 991-999.

Hammadi, A., Billard, C., Faussat, A.M., and Kolb, J.P. (2008). Stimulation of iNOS expression and apoptosis resistance in B-cell chronic lymphocytic leukemia (BCLL) cells through engagement of Toll-like receptor 7 (TLR-7) and NF-kappaB activation. Nitric Oxide 19, 138-145.

Hanak, V., Golbin, J.M., Hartman, T.E., and Ryu, J.H. (2008). High-resolution CT findings of parenchymal fibrosis correlate with prognosis in hypersensitivity pneumonitis. Chest 134, 133-138.

Hara, J., Ohtani, H., Matsumoto, T., Nakamura, S., Kitano, A., Arakawa, T., Nagura, H., and Kobayashi, K. (1997). Expression of costimulatory molecules B7-1 and B7-2 in macrophages and granulomas of Crohn's disease: demonstration of cell-to-cell contact with $\mathrm{T}$ lymphocytes. Laboratory investigation; a journal of technical methods and pathology 77, 175-184.

Hasan, S.A., Eksteen, B., Reid, D., Paine, H.V., Alansary, A., Johannson, K., Gwozd, C., Goring, K.A., Vo, T., Proud, D., and Kelly, M.M. (2013). Role of IL-17A and neutrophils in fibrosis in experimental hypersensitivity pneumonitis. J Allergy Clin Immunol 131, 1663-1673 e1665.

Hellings, P.W., Kasran, A., Liu, Z., Vandekerckhove, P., Wuyts, A., Overbergh, L., Mathieu, C., and Ceuppens, J.L. (2003). Interleukin-17 Orchestrates the Granulocyte Influx into Airways after Allergen Inhalation in a Mouse Model of Allergic Asthma. American Journal of Respiratory Cell and Molecular Biology 28, 42-50.

Hidmark, A., von Saint Paul, A., and Dalpke, A.H. (2012). Cutting edge: TLR13 is a receptor for bacterial RNA. J Immunol 189, 2717-2721. 
Ho, P.P., Fontoura, P., Ruiz, P.J., Steinman, L., and Garren, H. (2003). An immunomodulatory $\mathrm{GpG}$ oligonucleotide for the treatment of autoimmunity via the innate and adaptive immune systems. J Immunol 171, 4920-4926.

Hoek, R.M., Ruuls, S.R., Murphy, C.A., Wright, G.J., Goddard, R., Zurawski, S.M., Blom, B., Homola, M.E., Streit, W.J., Brown, M.H., et al. (2000). Downregulation of the macrophage lineage through interaction with OX2 (CD200). Science 290, 1768-1771.

Horton, M.A. (1997). The alpha v beta 3 integrin "vitronectin receptor". The international journal of biochemistry \& cell biology 29, 721-725.

Huaux, F., Liu, T., McGarry, B., Ullenbruch, M., and Phan, S.H. (2003). Dual roles of IL-4 in lung injury and fibrosis. J Immunol 170, 2083-2092.

Inoue, R., Nagino, T., Hoshino, G., and Ushida, K. (2011). Nucleic acids of Enterococcus faecalis strain EC-12 are potent Toll-like receptor 7 and 9 ligands inducing interleukin-12 production from murine splenocytes and murine macrophage cell line J774.1. FEMS immunology and medical microbiology 61, 94-102.

Ismail, T., McSharry, C., and Boyd, G. (2006). Extrinsic allergic alveolitis. Respirology (Carlton, Vic.) 11, 262-268.

Israel-Assayag, E., Dakhama, A., Lavigne, S., Laviolette, M., and Cormier, Y. (1999). Expression of costimulatory molecules on alveolar macrophages in hypersensitivity pneumonitis. Am J Respir Crit Care Med 159, 1830-1834.

Jakubzick, C., Choi, E.S., Joshi, B.H., Keane, M.P., Kunkel, S.L., Puri, R.K., and Hogaboam, C.M. (2003). Therapeutic attenuation of pulmonary fibrosis via targeting of IL-4- and IL-13-responsive cells. J Immunol 171, 2684-2693.

Jiang, W., Zhu, F.G., Bhagat, L., Yu, D., Tang, J.X., Kandimalla, E.R., La Monica, N., and Agrawal, S. (2013). A Toll-like receptor 7, 8, and 9 antagonist inhibits Th1 and Th17 responses and inflammasome activation in a model of IL-23-induced psoriasis. The Journal of investigative dermatology 133, 1777-1784.

Jimenez-Alvarez, L., Zuniga, J., Gaxiola, M., Checa, M., Becerril, C., Mendoza, F., Pardo, A., and Selman, M. (2010). Inflammatory response and dynamics of lung T cell subsets in Th1, Th2 biased and Th2 deficient mice during the development of hypersensitivity pneumonitis. Exp Mol Pathol 88, 407-415.

Joshi, A.D., Fong, D.J., Oak, S.R., Trujillo, G., Flaherty, K.R., Martinez, F.J., and Hogaboam, C.M. (2009). Interleukin-17-mediated immunopathogenesis in experimental hypersensitivity pneumonitis. Am J Respir Crit Care Med 179, $705-$ 716.

Kawai, T., and Akira, S. (2010). The role of pattern-recognition receptors in innate immunity: update on Toll-like receptors. Nat Immunol 11, 373-384. 
Kirby, A.C., Raynes, J.G., and Kaye, P.M. (2006). CD11b regulates recruitment of alveolar macrophages but not pulmonary dendritic cells after pneumococcal challenge. The Journal of infectious diseases 193, 205-213.

Kitamura, H., Cambier, S., Somanath, S., Barker, T., Minagawa, S., Markovics, J., Goodsell, A., Publicover, J., Reichardt, L., Jablons, D., et al. (2011). Mouse and human lung fibroblasts regulate dendritic cell trafficking, airway inflammation, and fibrosis through integrin alphavbeta8-mediated activation of TGF-beta. J Clin Invest $121,2863-2875$.

Koblansky, A.A., Jankovic, D., Oh, H., Hieny, S., Sungnak, W., Mathur, R., Hayden, M.S., Akira, S., Sher, A., and Ghosh, S. (2013). Recognition of profilin by Tolllike receptor 12 is critical for host resistance to Toxoplasma gondii. Immunity 38 , $119-130$.

Kokkarinen, J.I., Tukiainen, H.O., and Terho, E.O. (1992). Effect of corticosteroid treatment on the recovery of pulmonary function in farmer's lung. Am Rev Respir Dis $145,3-5$.

Kolaczkowska, E., and Kubes, P. (2013). Neutrophil recruitment and function in health and inflammation. Nat Rev Immunol 13, 159-175.

Krombach, F., Gerlach, J.T., Padovan, C., Burges, A., Behr, J., Beinert, T., and Vogelmeier, C. (1996). Characterization and quantification of alveolar monocytelike cells in human chronic inflammatory lung disease. The European respiratory journal 9, 984-991.

Kurup, V.P., Zacharisen, M.C., and Fink, J.N. (2006). Hypersensitivity pneumonitis. Indian J Chest Dis Allied Sci 48, 115-128.

Laan, M., Cui, Z.H., Hoshino, H., Lotvall, J., Sjostrand, M., Gruenert, D.C., Skoogh, B.E., and Linden, A. (1999). Neutrophil recruitment by human IL-17 via C-X-C chemokine release in the airways. J Immunol 162, 2347-2352.

Lee, C.G., Homer, R.J., Zhu, Z., Lanone, S., Wang, X., Koteliansky, V., Shipley, J.M., Gotwals, P., Noble, P., Chen, Q., et al. (2001). Interleukin-13 induces tissue fibrosis by selectively stimulating and activating transforming growth factor beta(1). J Exp Med 194, 809-821.

Li, X.D., and Chen, Z.J. (2012). Sequence specific detection of bacterial 23S ribosomal RNA by TLR13. eLife 1 , e00102.

Liu, Y., Bando, Y., Vargas-Lowy, D., Elyaman, W., Khoury, S.J., Huang, T., Reif, K., and Chitnis, T. (2010). CD200R1 Agonist Attenuates Mechanisms of Chronic Disease in a Murine Model of Multiple Sclerosis. The Journal of Neuroscience 30, 2025-2038. 
Lo Re, S., Dumoutier, L., Couillin, I., Van Vyve, C., Yakoub, Y., Uwambayinema, F., Marien, B., van den Brule, S., Van Snick, J., Uyttenhove, C., et al. (2010). IL17A-producing gammadelta $\mathrm{T}$ and $\mathrm{Th} 17$ lymphocytes mediate lung inflammation but not fibrosis in experimental silicosis. J Immunol 184, 6367-6377.

Mancuso, G., Gambuzza, M., Midiri, A., Biondo, C., Papasergi, S., Akira, S., Teti, G., and Beninati, C. (2009). Bacterial recognition by TLR7 in the lysosomes of conventional dendritic cells. Nat Immunol 10, 587-594.

Mangan, P.R., Harrington, L.E., O'Quinn, D.B., Helms, W.S., Bullard, D.C., Elson, C.O., Hatton, R.D., Wahl, S.M., Schoeb, T.R., and Weaver, C.T. (2006). Transforming growth factor-beta induces development of the $\mathrm{T}(\mathrm{H}) 17$ lineage. Nature 441, 231234.

Marim, F.M., Silveira, T.N., Lima, D.S., and Zamboni, D.S. (2010). A method for generation of bone marrow-derived macrophages from cryopreserved mouse bone marrow cells. PLoS One 5, e15263.

Mathur, R., Oh, H., Zhang, D., Park, S.G., Seo, J., Koblansky, A., Hayden, M.S., and Ghosh, S. (2012). A mouse model of Salmonella typhi infection. Cell 151, 590602.

Matsuno, Y., Ishii, Y., Yoh, K., Morishima, Y., Haraguchi, N., Kikuchi, N., Iizuka, T., Kiwamoto, T., Homma, S., Nomura, A., et al. (2007). Overexpression of GATA-3 protects against the development of hypersensitivity pneumonitis. Am J Respir Crit Care Med 176, 1015-1025.

McGeachy, M.J., Bak-Jensen, K.S., Chen, Y., Tato, C.M., Blumenschein, W., McClanahan, T., and Cua, D.J. (2007). TGF-beta and IL-6 drive the production of IL-17 and IL-10 by $\mathrm{T}$ cells and restrain $\mathrm{T}(\mathrm{H})-17$ cell-mediated pathology. Nat Immunol 8, 1390-1397.

Melton, A.C., Bailey-Bucktrout, S.L., Travis, M.A., Fife, B.T., Bluestone, J.A., and Sheppard, D. (2010). Expression of alphavbeta8 integrin on dendritic cells regulates Th17 cell development and experimental autoimmune encephalomyelitis in mice. J Clin Invest 120, 4436-4444.

Mitaka, K., Miyazaki, Y., Yasui, M., Furuie, M., Miyake, S., Inase, N., and Yoshizawa, Y. (2011). Th2-biased immune responses are important in a murine model of chronic hypersensitivity pneumonitis. Int Arch Allergy Immunol 154, 264-274.

Miyamoto, M., Prause, O., Sjostrand, M., Laan, M., Lotvall, J., and Linden, A. (2003). Endogenous IL-17 as a mediator of neutrophil recruitment caused by endotoxin exposure in mouse airways. J Immunol 170, 4665-4672.

Mortazavi, A., Williams, B.A., McCue, K., Schaeffer, L., and Wold, B. (2008). Mapping and quantifying mammalian transcriptomes by RNA-Seq. Nature methods 5, 621628. 
Mosser, D.M. (2003). The many faces of macrophage activation. Journal of Leukocyte Biology 73, 209-212.

Mosser, D.M., and Edwards, J.P. (2008). Exploring the full spectrum of macrophage activation. Nat Rev Immunol 8, 958-969.

Nagalakshmi, U., Waern, K., and Snyder, M. (2010). RNA-Seq: a method for comprehensive transcriptome analysis. Current protocols in molecular biology / edited by Frederick M. Ausubel ... [et al.] Chapter 4, Unit 4 11 11-13.

Nance, S., Cross, R., and Fitzpatrick, E. (2004). Chemokine production during hypersensitivity pneumonitis. Eur J Immunol 34, 677-685.

Nance, S., Cross, R., Yi, A.K., and Fitzpatrick, E.A. (2005). IFN-gamma production by innate immune cells is sufficient for development of hypersensitivity pneumonitis. Eur J Immunol 35, 1928-1938.

Nance, S.C., Yi, A.K., Re, F.C., and Fitzpatrick, E.A. (2008). MyD88 is necessary for neutrophil recruitment in hypersensitivity pneumonitis. J Leukoc Biol 83, 12071217.

Nicholas, S.A., Bubnov, V.V., Yasinska, I.M., and Sumbayev, V.V. (2011). Involvement of xanthine oxidase and hypoxia-inducible factor 1 in Toll-like receptor 7/8mediated activation of caspase 1 and interleukin-1 $\beta$. Cell Mol Life Sci 68, 151158.

Nicholas, S.A., Oniku, A.E., and Sumbayev, V.V. (2010). Myeloid cell death associated with Toll-like receptor 7/8-mediated inflammatory response. Implication of ASK1, HIF-1 alpha, IL-1 beta and TNF-alpha. Mol Immunol 48, 240-247.

Nie, H., Zheng, Y., Li, R., Guo, T.B., He, D., Fang, L., Liu, X., Xiao, L., Chen, X., Wan, B., et al. (2013). Phosphorylation of FOXP3 controls regulatory T cell function and is inhibited by TNF-alpha in rheumatoid arthritis. Nature medicine 19, 322328.

NIOSH. (2012). Work-related lung Disease Surveillance System (eWoRLD). . In Reference No. 2012F08-01 (U.S. Department of Health and Human Services, Public Health Service, Centers for Disease Control and Prevention, National Institute for Occupational Safety and Health, Division of Respiratory Disease Studies, Surveillance Branch. ).

Ohta, K., Takizawa, H., Suzuki, N., Ueda, T., Horiuchi, T., Okudaira, H., Shiga, J., and Ito, K. (1993). Hypersensitivity pneumonitis in the athymic nude mouse. Another evidence of T cell dependency. Chest 103,145S. 
Oldenburg, M., Krüger, A., Ferstl, R., Kaufmann, A., Nees, G., Sigmund, A., Bathke, B., Lauterbach, H., Suter, M., Dreher, S., et al. (2012). TLR13 Recognizes Bacterial 23S rRNA Devoid of Erythromycin Resistance-Forming Modification. Science $337,1111-1115$.

Oliver, B.G.G., Lim, S., Wark, P., Laza-Stanca, V., King, N., Black, J.L., Burgess, J.K., Roth, M., and Johnston, S.L. (2008). Rhinovirus exposure impairs immune responses to bacterial products in human alveolar macrophages. Thorax 63, 519525.

Paidassi, H., Acharya, M., Zhang, A., Mukhopadhyay, S., Kwon, M., Chow, C., Stuart, L.M., Savill, J., and Lacy-Hulbert, A. (2011). Preferential expression of integrin alphavbeta 8 promotes generation of regulatory $\mathrm{T}$ cells by mouse $\mathrm{CD} 103+$ dendritic cells. Gastroenterology 141, 1813-1820.

Pardo, A., Barrios, R., Gaxiola, M., Segura-Valdez, L., Carrillo, G., Estrada, A., Mejia, M., and Selman, M. (2000). Increase of lung neutrophils in hypersensitivity pneumonitis is associated with lung fibrosis. Am J Respir Crit Care Med 161, 1698-1704.

Park, Y., Oh, S.J., and Chung, D.H. (2009). CD4(+)CD25(+) regulatory T cells attenuate Hypersensitivity Pneumonitis by suppressing IFN-gamma production by CD4(+) and CD8(+) T cells. J Leukoc Biol 86, 1427-1437.

Pasare, C., and Medzhitov, R. (2003). Toll Pathway-Dependent Blockade of CD4+CD25+ T Cell-Mediated Suppression by Dendritic Cells. Science 299, 1033-1036.

Pasare, C., and Medzhitov, R. (2004). Toll-dependent control mechanisms of CD4 T cell activation. Immunity $21,733-741$.

Pechkovsky, D.V., Prasse, A., Kollert, F., Engel, K.M., Dentler, J., Luttmann, W., Friedrich, K., Muller-Quernheim, J., and Zissel, G. (2010). Alternatively activated alveolar macrophages in pulmonary fibrosis-mediator production and intracellular signal transduction. Clinical immunology (Orlando, Fla.) 137, 89-101.

Pesce, J.T., Ramalingam, T.R., Mentink-Kane, M.M., Wilson, M.S., El Kasmi, K.C., Smith, A.M., Thompson, R.W., Cheever, A.W., Murray, P.J., and Wynn, T.A. (2009). Arginase-1-expressing macrophages suppress Th2 cytokine-driven inflammation and fibrosis. PLoS pathogens 5, e1000371.

Plattner, F., Yarovinsky, F., Romero, S., Didry, D., Carlier, M.F., Sher, A., and SoldatiFavre, D. (2008). Toxoplasma profilin is essential for host cell invasion and TLR11-dependent induction of an interleukin-12 response. Cell Host Microbe 3, 77-87. 
Porcheray, F., Viaud, S., Rimaniol, A.C., Leone, C., Samah, B., Dereuddre-Bosquet, N., Dormont, D., and Gras, G. (2005). Macrophage activation switching: an asset for the resolution of inflammation. Clinical and experimental immunology 142, 481489.

Qi, R., Singh, D., and Kao, C.C. (2012). Proteolytic processing regulates Toll-like receptor 3 stability and endosomal localization. J Biol Chem 287, 32617-32629.

Rad, R., Ballhorn, W., Voland, P., Eisenächer, K., Mages, J., Rad, L., Ferstl, R., Lang, R., Wagner, H., Schmid, R.M., et al. (2009). Extracellular and intracellular pattern recognition receptors cooperate in the recognition of Helicobacter pylori. Gastroenterology 136, 2247-2257.

Ramaprakash, H., Ito, T., Standiford, T.J., Kunkel, S.L., and Hogaboam, C.M. (2009). Toll-like receptor 9 modulates immune responses to Aspergillus fumigatus conidia in immunodeficient and allergic mice. Infect Immun 77, 108-119.

Rathore, A., Sacristan, C., Ricklan, D.E., Flores Villanueva, P.O., and Stadecker, M.J. (1996). In situ analysis of B7-2 costimulatory, major histocompatibility complex class II, and adhesion molecule expression in schistosomal egg granulomas. Am J Pathol 149, 187-194.

Rijkers, E.S., de Ruiter, T., Baridi, A., Veninga, H., Hoek, R.M., and Meyaard, L. (2008). The inhibitory CD200R is differentially expressed on human and mouse $\mathrm{T}$ and $\mathrm{B}$ lymphocytes. Mol Immunol 45, 1126-1135.

Rio, D.C., Ares, M., Hannon, G.J., and Nilsen, T.W. (2010). Purification of RNA Using TRIzol (TRI Reagent). Cold Spring Harbor Protocols 2010, pdb.prot5439pdb.prot5439.

Rohmann, K., Tschernig, T., Pabst, R., Goldmann, T., and Dromann, D. (2011). Innate immunity in the human lung: pathogen recognition and lung disease. Cell and tissue research $343,167-174$.

Sakai, A., Koga, T., Lim, J.H., Jono, H., Harada, K., Szymanski, E., Xu, H., Kai, H., and Li, J.D. (2007). The bacterium, nontypeable Haemophilus influenzae, enhances host antiviral response by inducing Toll-like receptor 7 expression: evidence for negative regulation of host anti-viral response by CYLD. FEBS J 274, 3655-3668.

Salazar, E., and Knowles, J.H. (1964). AN ANALYSIS OF PRESSURE-VOLUME CHARACTERISTICS OF THE LUNGS. Journal of applied physiology: respiratory, environmental and exercise physiology 19, 97-104.

Schuyler, M., Gott, K., and Cherne, A. (2000). Mediators of hypersensitivity pneumonitis. The Journal of laboratory and clinical medicine 136, 29-38.

Schuyler, M., Gott, K., Cherne, A., and Edwards, B. (1997). Th1 CD4+ cells adoptively transfer experimental hypersensitivity pneumonitis. Cell Immunol 177, 169-175. 
Schuyler, M., Gott, K., Shopp, G., and Crooks, L. (1993). CD3+, CD4+, CD8-, Ia- T cells adoptively transfer murine experimental hypersensitivity pneumonitis. Chest $103,143 \mathrm{~S}-145 \mathrm{~S}$.

Selman, M., Lacasse, Y., Pardo, A., and Cormier, Y. (2010). Hypersensitivity pneumonitis caused by fungi. Proc Am Thorac Soc 7, 229-236.

Selman, M., Pardo, A., and King, T.E., Jr. (2012). Hypersensitivity pneumonitis: insights in diagnosis and pathobiology. Am J Respir Crit Care Med 186, 314-324.

Shi, C., and Pamer, E.G. (2011). Monocyte recruitment during infection and inflammation. Nat Rev Immunol 11, 762-774.

Shim, H.K., Kim, J.Y., Kim, M.J., Sim, H.S., Park, D.W., Sohn, J.W., and Kim, M.J. (2009). Legionella lipoprotein activates toll-like receptor 2 and induces cytokine production and expression of costimulatory molecules in peritoneal macrophages. Experimental \& molecular medicine 41, 687-694.

Simelyte, E., Criado, G., Essex, D., Uger, R.A., Feldmann, M., and Williams, R.O. (2008). CD200-Fc, a novel antiarthritic biologic agent that targets proinflammatory cytokine expression in the joints of mice with collagen-induced arthritis. Arthritis Rheum 58, 1038-1043.

Simonian, P.L., Roark, C.L., Wehrmann, F., Lanham, A.K., Diaz del Valle, F., Born, W.K., O'Brien, R.L., and Fontenot, A.P. (2009). Th17-polarized immune response in a murine model of hypersensitivity pneumonitis and lung fibrosis. J Immunol $182,657-665$.

Snelgrove, R., Goulding, J., Didierlaurent, A., Lyonga, D., Vekaria, S., Edwards, L., Gwyer, E., Sedgwick, J., Barclay, A., and Hussell, T. (2008). A critical function for CD200 in lung immune homeostasis and the severity of influenza infection. Nat Immunol 9, 1074 - 1083.

Soler, P., Boussaud, V., Moreau, J., Bergeron, A., Bonnette, P., Hance, A.J., and Tazi, A. (1999). In situ expression of B7 and CD40 costimulatory molecules by normal human lung macrophages and epithelioid cells in tuberculoid granulomas. Clinical and experimental immunology 116, 332-339.

Sorensen, L.N., Reinert, L.S., Malmgaard, L., Bartholdy, C., Thomsen, A.R., and Paludan, S.R. (2008). TLR2 and TLR9 synergistically control herpes simplex virus infection in the brain. J Immunol 181, 8604-8612.

Stein, M., Keshav, S., Harris, N., and Gordon, S. (1992). Interleukin 4 potently enhances murine macrophage mannose receptor activity: a marker of alternative immunologic macrophage activation. J Exp Med 176, 287-292. 
Stout, R.D., Suttles, J., Xu, J., Grewal, I.S., and Flavell, R.A. (1996). Impaired T cellmediated macrophage activation in CD40 ligand-deficient mice. J Immunol 156, $8-11$.

Strieter, R.M. (2008). What Differentiates Normal Lung Repair and Fibrosis? Proceedings of the American Thoracic Society 5, 305-310.

Suzuki, K., Suda, T., Naito, T., Ide, K., Chida, K., and Nakamura, H. (2005). Impaired toll-like receptor 9 expression in alveolar macrophages with no sensitivity to $\mathrm{CpG}$ DNA. Am J Respir Crit Care Med 171, 707-713.

Travis, M.A., Reizis, B., Melton, A.C., Masteller, E., Tang, Q., Proctor, J.M., Wang, Y., Bernstein, X., Huang, X., Reichardt, L.F., et al. (2007). Loss of integrin alpha(v)beta8 on dendritic cells causes autoimmunity and colitis in mice. Nature 449, 361-365.

Ultaigh, S.N., Saber, T.P., McCormick, J., Connolly, M., Dellacasagrande, J., Keogh, B., McCormack, W., Reilly, M., O'Neill, L.A., McGuirk, P., et al. (2011). Blockade of Toll-like receptor 2 prevents spontaneous cytokine release from rheumatoid arthritis ex vivo synovial explant cultures. Arthritis research \& therapy 13, R33.

Valencia, X., Stephens, G., Goldbach-Mansky, R., Wilson, M., Shevach, E.M., and Lipsky, P.E. (2006). TNF downmodulates the function of human CD4+CD25hi T-regulatory cells. Blood 108, 253-261.

Vanoirbeek, J.A., Rinaldi, M., De Vooght, V., Haenen, S., Bobic, S., Gayan-Ramirez, G., Hoet, P.H., Verbeken, E., Decramer, M., Nemery, B., and Janssens, W. (2010). Noninvasive and invasive pulmonary function in mouse models of obstructive and restrictive respiratory diseases. Am J Respir Cell Mol Biol 42, 96-104.

Vourlekis, J.S., Schwarz, M.I., Cherniack, R.M., Curran-Everett, D., Cool, C.D., Tuder, R.M., King, T.E., Jr., and Brown, K.K. (2004). The effect of pulmonary fibrosis on survival in patients with hypersensitivity pneumonitis. The American journal of medicine 116, 662-668.

Wagner, J.G., and Roth, R.A. (2000). Neutrophil migration mechanisms, with an emphasis on the pulmonary vasculature. Pharmacological reviews 52, 349-374.

Wang, X., Katwa, P., Podila, R., Chen, P., Ke, P.C., Rao, A.M., Walters, D.M., Wingard, C.J., and Brown, J.M. (2011). Multi-walled carbon nanotube instillation impairs pulmonary function in C57BL/6 mice. Particle and fibre toxicology 8, 24.

Weaver, C.T., Hatton, R.D., Mangan, P.R., and Harrington, L.E. (2007). IL-17 family cytokines and the expanding diversity of effector T cell lineages. Annu Rev Immunol 25, 821-852. 
Welker, L., Jorres, R.A., Costabel, U., and Magnussen, H. (2004). Predictive value of BAL cell differentials in the diagnosis of interstitial lung diseases. The European respiratory journal $24,1000-1006$.

Wick, G., Grundtman, C., Mayerl, C., Wimpissinger, T.F., Feichtinger, J., Zelger, B., Sgonc, R., and Wolfram, D. (2013). The immunology of fibrosis. Annu Rev Immunol 31, 107-135.

Wilson, M.S., Madala, S.K., Ramalingam, T.R., Gochuico, B.R., Rosas, I.O., Cheever, A.W., and Wynn, T.A. (2010). Bleomycin and IL-1beta-mediated pulmonary fibrosis is IL-17A dependent. J Exp Med 207, 535-552.

Witowski, J., Pawlaczyk, K., Breborowicz, A., Scheuren, A., Kuzlan-Pawlaczyk, M., Wisniewska, J., Polubinska, A., Friess, H., Gahl, G.M., Frei, U., and Jorres, A. (2000). IL-17 stimulates intraperitoneal neutrophil infiltration through the release of GRO alpha chemokine from mesothelial cells. J Immunol 165, 5814-5821.

Worthington, J.J., Fenton, T.M., Czajkowska, B.I., Klementowicz, J.E., and Travis, M.A. (2012). Regulation of TGFbeta in the immune system: an emerging role for integrins and dendritic cells. Immunobiology 217, 1259-1265.

Yamazaki, T., Yang, X.O., Chung, Y., Fukunaga, A., Nurieva, R., Pappu, B., MartinOrozco, N., Kang, H.S., Ma, L., Panopoulos, A.D., et al. (2008). CCR6 Regulates the Migration of Inflammatory and Regulatory $\mathrm{T}$ Cells. The Journal of Immunology 181, 8391-8401.

Yarovinsky, F., Zhang, D., Andersen, J.F., Bannenberg, G.L., Serhan, C.N., Hayden, M.S., Hieny, S., Sutterwala, F.S., Flavell, R.A., Ghosh, S., and Sher, A. (2005). TLR11 activation of dendritic cells by a protozoan profilin-like protein. Science 308, 1626-1629.

Yoshimori, T., Yamamoto, A., Moriyama, Y., Futai, M., and Tashiro, Y. (1991). Bafilomycin A1, a specific inhibitor of vacuolar-type $\mathrm{H}(+)$-ATPase, inhibits acidification and protein degradation in lysosomes of cultured cells. The Journal of biological chemistry 266, 17707-17712.

Zeuner, R.A., Ishii, K.J., Lizak, M.J., Gursel, I., Yamada, H., Klinman, D.M., and Verthelyi, D. (2002). Reduction of CpG-induced arthritis by suppressive oligodeoxynucleotides. Arthritis Rheum 46, 2219-2224.

Zhang, Y., Wang, F., Bhan, U., Huffnagle, G.B., Toews, G.B., Standiford, T.J., and Olszewski, M.A. (2010). TLR9 signaling is required for generation of the adaptive immune protection in Cryptococcus neoformans-infected lungs. Am J Pathol 177, 754-765. 
Zhou, L., Ivanov, I.I., Spolski, R., Min, R., Shenderov, K., Egawa, T., Levy, D.E., Leonard, W.J., and Littman, D.R. (2007). IL-6 programs T(H)-17 cell differentiation by promoting sequential engagement of the IL-21 and IL-23 pathways. Nat Immunol 8, 967-974. 


\section{APPENDIX. TRANSCRIPTOME ANALYSIS}

\section{Introduction}

The advancement in RNA sequencing (RNAseq) technology has made this technique faster and more affordable than ever. Analysis of gene transcription by RTPCR is limited to preselected genes, whereas RNAseq measures the expression of all transcripts in the sample. Previous studies in our lab identified MyD88 as a critical signaling adaptor molecule for the induction of inflammatory cytokine and chemokine production in response to SR (Nance et al., 2008). Studies during the acute phase found many cytokines and chemokines induced by SR were partially dependent on TLRs 2 and 9. In addition, TLR7 may play a role in SR induced cytokine production in spleen cells. However, other non-MyD88-dependent pattern recognition receptors may also contribute to the immune response to SR.

NOD-like receptors (NLRs) are a family of cytosolic pattern recognition receptors. Like TLRs, NLRs recognize pathogen associated molecular patterns (PAMPs) as well as danger associated molecular patterns (DAMPs) such as molecules associated with tissue damage such as uric acid (Gasse et al., 2009). NOD1 and NOD2 are NLRs that recognize the breakdown products of the bacterial cell wall, meso-diaminopimelic acid (meso-DAP) and muramyl dipeptide (MDP) respectively. Bacterial cell walls typically contain MDP, but gram negative and some gram positive bacterial cell walls also contain meso-DAP. SR is a gram positive actinomycete, which has a type IV cell wall structure that contains meso-DAP, arabinose and galactose, but not mycolic acids (Embley et al., 1988), and can therefore potentially stimulate NOD1 and NOD2.

To explore changes in global gene transcription after SR stimulation, bone marrow derived dendritic cells (BMDCs) from WT, TLR2 $/ 9^{-/-}, \mathrm{MyD} 88^{-/-}, \mathrm{TLR}^{-/-}$and $\mathrm{NOD}_{2}{ }^{-/}$mice were stimulated with SR in vitro. We hypothesize loss of MyD88, TLRs 2 and 9, TLR7, and NOD2 will lead to a decrease in global gene transcription. Comparing levels of transcription between WT and KO cells will identify genes that are potentially dependent TLRs 2 and 9, TLR7, NOD2, and signaling through MyD88.

\section{Methods}

\section{Bone Marrow Harvest and Storage}

Bone marrow cells were harvested from the femur and tibia of WT, $\mathrm{TLR} 7^{-/-}$, $\mathrm{TLR} 2 / 9^{-/-}, \mathrm{MyD}^{-1-} 8^{-/}$, and NOD2 ${ }^{-/-}$mice. Mice were sacrificed and fur completely wetted down with $70 \%$ ethanol. The fur, skin, and large muscle tissue was removed from the hind legs. Legs were removed at the hip and the paw removed at the joint to prevent cutting the femur and tibia. The bones were sprayed with ethanol and placed in media on ice until all mice were processed. In a sterile hood, any remaining flesh was removed 
from the bones. The epiphysis was cut and bone marrow flushed with sterile PBS into a $50 \mathrm{~mL}$ tube using a 26 gauge $1 / 2$ inch needle and syringe. The bone marrow was centrifuged at $400 \mathrm{~g}$ and supernatant decanted. The bone marrow cells were pipetted up and down to break up clumps and washed with sterile PBS and pelleted at $400 \mathrm{~g}$. Supernatant was discarded and RBCs lysed with RBC lysis buffer. Bone marrow cells were passed through a $70 \mu \mathrm{m}$ cell strainer (BD Biosciences, San Jose, CA) to obtain a single cell suspension. Cells were pelleted at $400 \mathrm{~g}$ and resuspended in media. Cells were counted by trypan dye exclusion on a hemocytometer and brought to a concentration of $6 \times 10^{6}$ cells $/ \mathrm{mL}$. Bone marrow cells were either plated immediately or reconstituted in freezing media $(90 \%$ heat inactivated $\mathrm{FCS}, 10 \% \mathrm{DMSO})$ and frozen at $-80^{\circ} \mathrm{C}$ and transferred to liquid nitrogen for long term storage (Marim et al., 2010). To thaw bone marrow progenitor cells for BMDC differentiation, bone marrow progenitor cells were rapidly thawed in a $37^{\circ} \mathrm{C}$ water bath and were washed with media to removed DMSO.

\section{Bone Marrow Derived Dendritic Cell (BMDC) Differentiation}

To generate dendritic cells, $5 \times 10^{6}$ bone marrow progenitor cells from WT, $\mathrm{MyD}_{8} 8^{-/-}, \mathrm{TLR} 2 / 9^{-/-}, \mathrm{TLR}^{-/-}$, and $\mathrm{NOD}^{-/-}$mice were plated in $15 \mathrm{~mL} \mathrm{BMDC}$ differentiation media containing $35 \%$ F10 media (Table A-1) in a $15 \mathrm{~cm}$ cell culture dish. The cells were cultured 8 days. On day 3 , an additional $15 \mathrm{~mL}$ of BMDC differentiation media containing $35 \%$ F10 media was added. On day $6,15 \mathrm{~mL}$ media was removed from the plate and spun down at $400 \mathrm{~g}$ for 4 minutes. The supernatant was decanted, and $15 \mathrm{~mL}$ fresh BMDC differentiation media containing 35\% F10 media was added to the pellet and returned to the plate. On day 8 , the tissue culture plates were gently washed with media 2 times to collect loosely adherent cells. The harvested cells were pelleted at $400 \mathrm{~g}$ for 4 minutes, and resuspended in fresh BMDC cultivation media containing 17.5\% F10 media. An aliquot of cells was used to determine the percentage of conventional DC $\left(\mathrm{CD} 11 \mathrm{~b}^{+} \mathrm{CD} 11 \mathrm{c}^{+} \mathrm{Gr}-1^{-}\right)$, monocyte derived inflammatory DC $\left(\mathrm{CD} 11 \mathrm{~b}^{+} \mathrm{CD} 11 \mathrm{c}^{+} \mathrm{Gr}-1^{-}\right)$, inflammatory monocytes $\left(\mathrm{CD} 11 \mathrm{~b}^{+} \mathrm{CD} 11 \mathrm{c}^{-} \mathrm{Gr}-1^{+}\right)$, and monocyte/macrophage $\left(\mathrm{CD} 11 \mathrm{~b}^{+}\right.$ $\mathrm{CD} 11 \mathrm{c}^{-} \mathrm{Gr}-1^{-}$) was determined by flow cytometry based upon the gating strategy of Bourgeois et al. (Bourgeois et al., 2011). The BMDC preparation was 89-95\% conventional DCs, monocyte derived inflammatory DCs, and inflammatory monocytes.

\section{BMDC Stimulation}

BMDCs $\left(2.5 \times 10^{6}\right.$ cells/well $)$ were plated in a 6 well plate. Cells were stimulated with media or $0.05 \mathrm{mg} / \mathrm{mL}$ SR for 12 hours. The next day cells from all wells were harvested by scraping, and washed with PBS. Cells were lysed with TRIzol for poly-A RNA isolation and transcriptome analysis. 
Table A-1. BMDC media.

\begin{tabular}{|c|c|c|c|c|}
\hline Reagent & $\begin{array}{c}\text { Differentiation } \\
\text { media }\end{array}$ & $\begin{array}{c}\text { Cultivation } \\
\text { media }\end{array}$ & Manufacturer & Location \\
\hline RPMI & $\mathrm{X}$ & $\mathrm{X}$ & $\begin{array}{l}\text { Cellgro, } \\
\text { Mediatech Inc. }\end{array}$ & Manassas, VA \\
\hline $\begin{array}{l}10 \% \text { heat } \\
\text { inactivated FCS }\end{array}$ & $\mathrm{X}$ & $X$ & $\begin{array}{l}\text { Hyclone, } \\
\text { Thermo } \\
\text { Scientific }\end{array}$ & Logan, UT \\
\hline 2mM glutamine & $X$ & $X$ & $\begin{array}{l}\text { Cellgro, } \\
\text { Mediatech Inc. }\end{array}$ & Manassas, VA \\
\hline $\begin{array}{l}\text { 100IU/mL } \\
\text { penicillin }\end{array}$ & $\mathrm{X}$ & $\mathrm{X}$ & $\begin{array}{l}\text { Cellgro, } \\
\text { Mediatech Inc. }\end{array}$ & Manassas, VA \\
\hline $\begin{array}{l}100 \mu \mathrm{g} / \mathrm{mL} \\
\text { streptomycin }\end{array}$ & $\mathrm{X}$ & $\mathrm{X}$ & $\begin{array}{l}\text { Cellgro, } \\
\text { Mediatech Inc. }\end{array}$ & Manassas, VA \\
\hline $\begin{array}{l}1 \mu \mathrm{L} \beta \text { - } \\
\text { mercaptoethanol }\end{array}$ & $\mathrm{X}$ & $X$ & $\begin{array}{l}\text { Fisher } \\
\text { Scientific }\end{array}$ & Waltham, MA \\
\hline F10 media & $35 \%$ & $17.5 \%$ & & \\
\hline
\end{tabular}




\section{RNA Isolation}

Culture media was removed completely and Trizol (Invitrogen) was added to the plate and allowed to incubate for 5 minutes. Trizol was then transferred to a microcentrifuge tube for RNA isolation. The aqueous layer was separated using chloroform. Glycogen was added to the aqueous RNA then both were precipitated with isopropanol. The RNA/glycogen pellet was washed with $75 \%$ ethanol twice. The $\mathrm{RNA} /$ glycogen pellet was then dissolved in water and heated at $55^{\circ} \mathrm{C}$ for 10 minutes.

\section{Poly-A RNA Enrichment}

RNA isolated from BMDCs was precipitated a second time using ammonium acetate, glycogen, and $100 \%$ ethanol to remove additional salt. The pellet was washed with $70 \%$ ethanol, and resuspended in nuclease free water. The RNA was then incubated with Oligo(dT) resin (Poly-A purist kit, Ambion, Naugatuck, CT). The resin was washed to remove unbound ribosomal RNA and other sources of non-messenger RNA. The poly-A mRNA was eluted with THE RNA storage solution and re-precipitated with ammonium acetate, glycogen, and $100 \%$ ethanol. The pellet was washed with $70 \%$ ethanol, and resuspended in nuclease free water for a second round of Poly-A purification.

An aliquot of total RNA isolated for transcriptome analysis was run on a eukaryote nano chip in an Agilent 2100 Bioanalyzer (Agilent Technologies, Santa Clara, CA) by personnel in the Molecular Resource Center of UTHSC. The RNA integrity number (RIN) for all samples was above 9.0. To check for ribosomal RNA contamination, an aliquot of purified poly-A RNA samples were again run on the Agilent Bioanalyzer to verify the $18 \mathrm{~S}$ and $28 \mathrm{~S}$ ribosomal bands were absent. One sample, after two rounds of poly-A RNA isolation, still had a small ribosomal RNA peak and required a third round of poly-A RNA isolation.

\section{BMDC Transcriptome Analysis}

\section{Poly-A RNA Fragmentation and Reverse Transcription}

Poly-A RNA samples were prepared for RNA sequencing (RNA-seq) by personnel in the Molecular Resource Center of UTHSC (Mortazavi et al., 2008; Nagalakshmi et al., 2010). Using the SOLiD Total RNA-seq Kit (Applied Biosystems, Carlsbad, CA), the purified poly-A RNA was fragmented and cleaned using a spin column. The fragmented RNA was then run on a pico chip on the Agilent Bioanylizer to assess the fragmentation size range. The RNA fragments were hybridized to adaptors, then reverse transcribed to cDNA. 
The cDNA was washed and underwent two rounds of size selection to select for cDNA products greater than $150 \mathrm{bp}$. The cDNA was then amplified using different barcoded primers for each sample and purified to remove unincorporated primers and run on a DNA 1000 chip in the Agilent Bioanalyzer for a smear analysis. (A smear analysis determines the percentage of cDNA products that fall between $25-200 \mathrm{bp}$. Less than $50 \%$ is considered optimal.)

\section{SOLiD System Sequencing}

The cDNA from WT, MyD $88^{-/-}, \mathrm{TLR}_{2} / 9^{-/-}, \mathrm{TLR}^{-/}{ }^{-}$, and $\mathrm{NOD} 2^{-/}$SR stimulated BMDCs was sequenced on a SOLiD 5500xl (Applied Biosystems, Carlsbad, CA) by personnel in the Molecular Resource Center of UTHSC. Sequences were aligned to mouse $\mathrm{mm} 9$ annotated reference genome using Lifescope genomic analysis software. The read count for each gene was normalized for transcript length in nucleotides and the total number of reads in the sample by calculating the reads per kilobase of exon model per million mapped reads (RPKM).

$$
R P K M=\left(\text { Read } \text { Count }_{\text {gene }} \times 10^{9}\right) /\left(\text { Length }_{\text {Gene transcript }}\right)\left(\text { Read Count }_{\text {Total }}\right)
$$

The fold induction was calculated by dividing the RPKM of SR stimulated WT and KO BMDCs by WT media. A cut off value of 5x or greater fold induction was used to identify genes upregulated by SR stimulation. Out of the total number of genes upregulated in WT SR stimulated BMDCs, a fold reduction was determined by dividing the RPKM of WT SR stimulated BMDCs by the RPKM of KO SR stimulated BMDCs. A cut off value of $5 \mathrm{x}$ or greater was used to identify which genes were dependent on MyD88, TLRs 2 and 9, TLR7, and NOD2. The list of upregulated and downregulated

genes was entered into the MGI Gene GO_Slim Chart Tool (http://www.informatics.jax.org/gotools/MGI_GO_Slim_Chart.html) for biological process and function analysis to identify commonalities among genes upregulated by SR.

\section{Results}

To assess the global changes in cell transcription in response to SR, total RNA was harvested from media or SR stimulated BMDCs. BMDCs were polarized from bone marrow harvested from WT, MyD88 $8^{-/}$, TLR2 $/ 9^{-/-}$, TLR7 ${ }^{-/}$, and NOD2 ${ }^{-1-}$ mice and cultured in the presence of F10 media. The cells were polarized for 8 days and stimulated with SR for 12 hours. Poly-A RNA was isolated then sequenced for transcriptome analysis. The read counts were normalized to transcript size by calculating the reads per kilobase of exon model per million mapped reads (RPKM). The number of genes upregulated by SR stimulation was calculated by dividing the RPKM of SR stimulated BMDCs by the RPKM of WT media BMDCs (Figure A-1). SR stimulation upregulated 648 genes $\geq 5 \mathrm{x}$ in WT BMDCs compared to media. The number of genes upregulated in $\mathrm{TLR}^{-/-}$and NOD2 ${ }^{-/-}$SR stimulated BMDCs were similar to WT SR stimulated BMDCs 

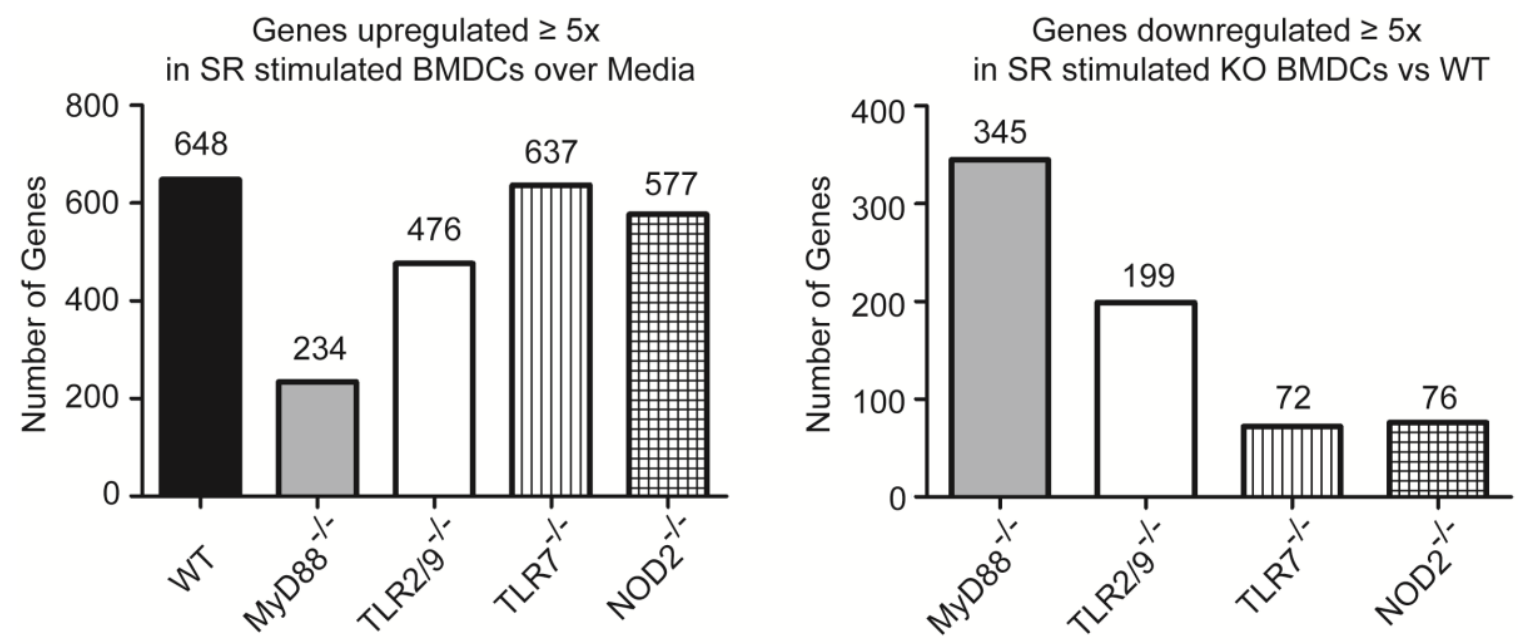

Figure A-1. Changes in gene expression in WT, $\mathrm{MyD88}^{-/}, \mathrm{TLR2}^{-/-}, \mathrm{TLR}^{-/-}$, NOD2 $^{-/-}$BMDCs stimulated with SR in vitro.

WT, MyD88 $8^{-/-}$, TLR2 $/ 9^{-/-}, \mathrm{TLR}^{-/-}$, and NOD2 ${ }^{-/-}$BMDCs were stimulated with SR $(0.05 \mathrm{mg} / \mathrm{mL})$ for 12 hours. RNA was harvested and enriched for poly-A RNA for RNA sequencing. Sequences were aligned to the mouse genome. Transcripts were mapped to a total of 24226 genes. The read count for the genes expressed in each sample was normalized for transcript length by calculating the reads per kilobase of exon model per million mapped reads (RPKM). The number of genes with RPKM values $\geq 5 \mathrm{x}$ higher in SR stimulated BMDMs compared to media stimulated BMDMs was calculated. Out of the genes upregulated $\geq 5 \mathrm{x}$ in WT SR stimulated BMDMs over media, the number of genes that had RPKM values $\geq 5 \mathrm{x}$ lower in MyD88 ${ }^{-/}$, TLR2 $/ 9^{-/-}$, TLR $7^{-/-}$, and NOD2 $2^{-/-}$ SR stimulated BMDMs was calculated. 
(637 and 577 genes respectively). TLR2/9 $9^{-/}$BMDCs stimulated with SR upregulated fewer genes than WT SR stimulated BMDCs (476 vs 648 genes). However, MyD88 ${ }^{-/-}$ SR stimulated BMDCs upregulated about $1 / 3$ the number of genes compared to WT SR (234 genes vs 648 genes). Out of the 648 genes upregulated $\geq 5 \mathrm{x}$ in WT BMDCs stimulated with SR compared to WT media, approximately half of those genes (345 genes) were downregulated $\geq 5 \mathrm{x}$ in MyD88 $8^{-/-}$BMDCs. In contrast, approximately one third of those genes (199 genes) were downregulated $\geq 5 \mathrm{x}$ in TLR2/9 $9^{-/-}$BMDCs. However, in $\mathrm{TLR}^{-/-}$and $\mathrm{NOD}^{-/-} \mathrm{SR}$ stimulated BMDCs, only 72 and 76 genes respectively were downregulated $>5 \mathrm{x}$ compared to WT SR.

To investigate the biological process of genes upregulated in response to SR, a list of genes that were upregulated $\geq 5 \mathrm{x}$ in SR stimulated BMDCs over WT media was entered into the MGI Gene Ontology GO_Slim chart tool, an online analysis program which groups genes based on shared biological process or function. Biological processes were grouped into 13 categories, and the percentage of genes in each category was not dramatically different in $\mathrm{WT}, \mathrm{TLR}^{-1-}$, and $\mathrm{NOD}^{-/-}$SR stimulated BMDCs (Table A-2). The percentage of genes involved in cell adhesion, cell-cell signaling, cell cycle and proliferation, and death was slightly reduced in TLR $2 / 9^{-/-}$BMDCs. The percentage was further reduced in MyD88 ${ }^{-/-}$BMDCs in cell adhesion, cell-cell signaling, cell cycle and proliferation, death, cell organization and biogenesis, protein metabolism transport, and developmental processes. Out of the upregulated genes, many shared a biological function related to the induction of an immune response, such as cytokines, chemokines, cytokine and chemokine receptors, and integrins (Table A-3). In addition, growth factors and growth factor receptors were upregulated, as well as genes potentially involved in the fibrotic response such as extracellular matrix constituents and metalloproteinases. Altogether, the results suggest a majority of genes require a signal through a MyD88-dependent receptor to upregulate gene transcription after SR stimulation, and some genes are dependent on TLRs 2 or 9. The loss of TLR7 or NOD2 alone was not enough to dramatically alter cellular gene transcription.

\section{Discussion}

The transcriptome analysis revealed MyD88-dependent receptors to be crucial for SR induced upregulation of transcription. MyD $88^{-/-}$BMDCs upregulated fewer genes in response to SR and had the greatest number of genes downregulated $>5 \mathrm{x}$ compared to WT SR exposed BMDCs. TLRs 2 and 9 were revealed to be partially responsible for the MyD88-dependent induction of transcription; however, in agreement with studies during the acute phase, other MyD88-dependent receptors contribute to the response to SR. The loss of TLR7 or NOD2 alone was not enough to dramatically alter gene transcription in BMDCs, suggesting they play only a minor role in the innate immune response to SR.

Although the transcriptome analysis was limited in sample size, the data can be used to identify candidate genes for further analysis. One of the genes highly upregulated in BMDCs in response to SR stimulation was integrin $\beta 8$ (WT SR RPKM 15.66 vs WT 
Table A-2. Percentage of genes upregulated $\geq 5 x$ over WT media that share a biological process.

\begin{tabular}{|c|c|c|c|c|c|}
\hline Biological process & WT SR & $\begin{array}{l}\text { MyD88 }^{-/-} \\
\text {SR }\end{array}$ & $\begin{array}{l}\text { TLR2/9 }^{-1-} \\
\text { SR }\end{array}$ & $\begin{array}{l}\text { TLR7 } 7^{-1-} \\
\text { SR }\end{array}$ & $\begin{array}{l}\mathrm{NOD2}^{-l-} \\
\text { SR }\end{array}$ \\
\hline Cell adhesion & $6.64 \%$ & $3.42 \%$ & $4.83 \%$ & $5.49 \%$ & $6.41 \%$ \\
\hline Cell-cell signaling & $6.17 \%$ & $4.27 \%$ & $4.62 \%$ & $5.97 \%$ & $6.76 \%$ \\
\hline $\begin{array}{l}\text { Cell cycle and } \\
\text { proliferation }\end{array}$ & $14.04 \%$ & $7.69 \%$ & $9.87 \%$ & $12.24 \%$ & $13.69 \%$ \\
\hline Death & $9.72 \%$ & $5.13 \%$ & $7.56 \%$ & $10.68 \%$ & $11.27 \%$ \\
\hline $\begin{array}{l}\text { Cell organization and } \\
\text { biogenesis }\end{array}$ & $15.12 \%$ & $12.39 \%$ & $14.08 \%$ & $14.76 \%$ & $16.98 \%$ \\
\hline Protein metabolism & $17.13 \%$ & $12.82 \%$ & $15.76 \%$ & $15.07 \%$ & $15.42 \%$ \\
\hline DNA metabolism & $1.85 \%$ & $2.14 \%$ & $2.10 \%$ & $2.20 \%$ & $2.25 \%$ \\
\hline RNA metabolism & $10.80 \%$ & $9.40 \%$ & $11.13 \%$ & $11.15 \%$ & $11.61 \%$ \\
\hline $\begin{array}{l}\text { Other metabolic } \\
\text { processes }\end{array}$ & $16.67 \%$ & $15.81 \%$ & $15.55 \%$ & $15.86 \%$ & $15.94 \%$ \\
\hline Stress response & $15.59 \%$ & $14.10 \%$ & $17.86 \%$ & $16.95 \%$ & $16.46 \%$ \\
\hline Transport & $16.20 \%$ & $12.39 \%$ & $14.71 \%$ & $15.07 \%$ & $15.77 \%$ \\
\hline $\begin{array}{l}\text { Developmental } \\
\text { processes }\end{array}$ & $23.61 \%$ & $19.23 \%$ & $20.59 \%$ & $21.51 \%$ & $24.96 \%$ \\
\hline Signal transduction & $20.37 \%$ & $21.79 \%$ & $18.07 \%$ & $19.31 \%$ & $19.06 \%$ \\
\hline Total genes & 648 & 234 & 476 & 637 & 577 \\
\hline
\end{tabular}


Table A-3. Genes upregulated $\geq 5 x$ over WT media BMDCs that share a biological function related to an immune response or fibrosis.

\section{Biological function}

Cytokines

Chemokines

Cytokine and chemokine receptors

Growth factors

Growth factor receptors and binding proteins

Extracellular matrix constituents

Metalloproteinases

Integrins and integrin binding proteins
16

\section{Number of genes}

20

12

9

13

9

6

8 
media RPKM 0.05, upregulation 313x), and the upregulation was dependent on MyD88 (RPKM 0.56 vs WT SR RPKM 15.66, downregulation 28x) and TLRs 2 and 9 (RPKM 1.94 vs WT SR RPKM 15.66, downregulation 8x). Integrins are a family of adhesion molecules that bind to the extracellular matrix and are critical for cellular trafficking of immune cells. They consist of two chains, an alpha chain and a beta chain, and the pairing determines substrate specificity. Integrin $\alpha v$ pairs with $\beta 3$ to form the vitronectin receptor, which binds vitronectin and other components of the extracellular matrix (Horton, 1997). Integrin $\alpha v$ can also pair with $\beta 8$ to activate TGF $\beta$. TGF $\beta$ is secreted in an inactive form bound to the latency associated peptide (LAP). TGF $\beta$ activating integrins such as $\alpha v \beta 8$ bind LAP through an RGD-motif to release active TGF $\beta$ (reviewed in Worthington et al., 2012). Previous studies have shown that $\alpha \mathrm{v}$ and specifically $\alpha v \beta 8$ are required for TGF $\beta$ activation and polarization of Tregs and Th17 cells in a mouse model of EAE and colitis (Acharya et al., 2010; Melton et al., 2010; Paidassi et al., 2011; Travis et al., 2007). The upregulation of $\alpha v \beta 8$ on fibroblasts has also been associated cytokine and chemokine secretion and the development of lung fibrosis in a mouse model of asthma and COPD (Kitamura et al., 2011). Importantly, treatment of mice with a cyclic RGD peptide protected mice from the development of EAE, and blocked the activation of TGF $\beta$ and the polarization of Th17 cells in vitro (Acharya et al., 2010). Further studies to confirm the upregulation of $\alpha v \beta 8$ in SR exposed mice are necessary, but may reveal a critical mediator of the Th17 response and lung fibrosis in HP. 


\section{VITA}

Kelly Andrews was born in 1981 in Lansing, Michigan. She graduated from Western Michigan University in 2004 with a major in Biomedical Sciences, and a minor in Chemistry and Environmental Sciences. She continued to take math and physics classes at Lansing Community College in 2005 and 2006. In 2007, she took classes in biochemistry and immunology at Michigan State University and volunteered in the lab of Dr. Animesh Sinha. In 2008, she entered the Integrated Program in Biomedical Sciences (IPBS) at the University of Tennessee Health Science Center. She joined the laboratory of Dr. Elizabeth Fitzpatrick where she investigated the role of toll-like receptors 2 and 9 in the pathogenesis of hypersensitivity pneumonitis. She graduated in December of 2013 with a PhD in immunology. 